University of Louisville

ThinkIR: The University of Louisville's Institutional Repository

Electronic Theses and Dissertations

$12-2011$

\title{
Tumor-targeting aptamers for the treatment of prostate cancer.
}

Enid W. Choi

University of Louisville

Follow this and additional works at: https://ir.library.louisville.edu/etd

\section{Recommended Citation}

Choi, Enid W., "Tumor-targeting aptamers for the treatment of prostate cancer." (2011). Electronic Theses and Dissertations. Paper 245.

https://doi.org/10.18297/etd/245

This Doctoral Dissertation is brought to you for free and open access by ThinkIR: The University of Louisville's Institutional Repository. It has been accepted for inclusion in Electronic Theses and Dissertations by an authorized administrator of ThinkIR: The University of Louisville's Institutional Repository. This title appears here courtesy of the author, who has retained all other copyrights. For more information, please contact thinkir@louisville.edu. 


\title{
“TUMOR-TARGETING APTAMERS FOR THE TREATMENT OF PROSTATE CANCER"
}

\author{
Enid W. Choi, B.S. \\ Massachusetts Institute of Technology, 2000 \\ A dissertation submitted to the faculty of the School of Medicine \\ University of Louisville \\ in partial fulfillment of the requirements for the degree of Doctor of Philosophy \\ Department of Biochemistry and Molecular Biology \\ University of Louisville \\ Louisville, Kentucky
}

December 2011 
"Tumor-Targeting Aptamers for the Treatment of Prostate Cancer"

$$
\text { by }
$$

Enid W. Choi, B.S.

Massachusetts Institute of Technology, 2000

A dissertation approved on

$11 / 3 / 2011$

by, the undersigned Dissertation Committee:

Paula Bates, Ph.D., Dissertation Director

Douglas Dean, Ph.D.

Carolyn Klinge, Ph.D.

Russell Prough, Ph.D.

Brian Wattenberg, Ph.D. 


\section{ACKNOWLEDGMENTS}

Paula Bates, Ph.D.

Kavitha Yaddanapudi, Ph.D.

University of Louisville M.D./Ph.D. Program

$\begin{array}{lc}\text { Maria Barnes } & \text { Derik Pridmore } \\ \text { Lavona Casson } & \text { E. Merit Reyes-Reyes } \\ \text { Christine Choi } & \text { Kara Sedoris } \\ \text { Jungja Choi } & \text { M. Bradley Spurlock } \\ \text { Logan Choi } & \text { Yun Teng } \\ \text { Ashraful Islam } & \text { Sheila Thomas } \\ \text { M. Tariq Malik } & \text { Christopher Worth } \\ \text { Lalitha Nayak } & \text { Ewa Zuba-Surma }\end{array}$




\begin{abstract}
“TUMOR-TARGETING APTAMERS FOR THE TREATMENT

OF PROSTATE CANCER"
\end{abstract}

Enid W. Choi, B.S.

December 2011

Prostate cancer is the most common type diagnosed among men in the United States. Random oligodeoxynucleotide (ODN) libraries are used to generate DNA aptamers by systematic evolution of ligands by exponential enrichment (SELEX). We explored the utility of guanine-rich libraries, and found that some had cancer-selective antiproliferative activity that generally correlated with G-quadruplex formation, nuclease resistance, and enhanced cellular uptake. We identified several proteins by mass spectrometry that bind to GC and cytosine ODNs. We compared the GT library with the classic random library for SELEX of whole prostate cancer cells and identified a new aptamer from the GT library with enhanced nuclease resistance and antiproliferative activity. We present evidence that AS1411, a known G-rich anticancer aptamer, inhibits prostate tumor initiating cells. This body of work supports the hypothesis that G-rich ODNs are well suited to the development of new aptamers in the treatment of cancer. 


\section{TABLE OF CONTENTS}

\section{CHAPTER 1}

Introduction to novel concepts in oncology research: Aptamers and tumor initiating cells

- Background

- Rationale and purpose

- Hypothesis and objective

- Specific aims

\section{CHAPTER 2}

Cancer-selective antiproliferative activity is a general property of some G-rich oligodeoxynucleotide libraries

- Introduction

- Conclusions

\section{CHAPTER 3}

Protein targets of the GC oligodeoxynucleotide $26 \mathrm{mer}$ library and poly-deoxycytosine 26mer

- Introduction

- Materials and methods 
- Results and discussion

- Conclusions

\section{CHAPTER 4}

Identification of a new anti-prostate cancer aptamer by SELEX

- Introduction

- Materials and methods

- Results and discussion

- Conclusions

\section{CHAPTER 5}

Evidence that AS1411 (a G-rich aptamer) inhibits the growth of tumor initiating cells

- Introduction

- Materials and methods

- Results and discussion

- Conclusions

\section{APPENDICES}

- Appendix A: Manuscript from Nucleic Acids Research 
- Appendix B: SELEX sequences from N and GT library using PC3 prostate

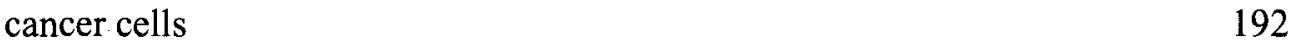

- Appendix C: Microarray Analysis of Changes in Gene Expression in DU145 Cells Treated with AS1411

- Appendix D: Abbreviations and Symbols 


\section{LIST OF FIGURES}

\section{CHAPTER 1}

1. Schematic of SELEX workflow 9

$\begin{array}{ll}\text { 2. Cancer stem cells and clinical implications } & 14\end{array}$

\section{CHAPTER 3}

1. Precipitation of proteins from PC3 whole cell lysate with biotinylated $\begin{array}{ll}\text { oligodeoxynucleotide libraries } & 58\end{array}$

\section{CHAPTER 4}

1. Radioactive PCR of final wash and lysate 93

2. Radioactive PCR from sequential rounds of SELEX 93

3. Cellular uptake of FAM-labeled oligodeoxynucleotides 96

4. MTT assay of candidate aptamers compared to parent library and AS1411 on $\begin{array}{ll}\text { different cell types } & 102\end{array}$

5. CD spectra of candidate aptamers and parent libraries 106

6. Stability of candidate aptamers and parent libraries 106

7. Protein binding of candidate aptamers and parent libraries in PC3 cytoplasmic extract

\section{CHAPTER 5}

1. MTT assay of experimental drugs on DU145 cells after 72 hours

2. Cell cycle and cytotoxic effects of AS1411 on bulk DU145 cells 
3. Baseline levels of TIC markers in different types of DU145 cells

4. Heterogeneity of DU145 monolayer versus spherocytes cells

5. Effect AS1411 on percentage of $\mathrm{CD} 24^{-} / \mathrm{CD} 44^{+}$DU145 cells at various time points and concentrations

6. Effect of $10 \mu \mathrm{M}$ AS1411 on DU145 spherocytes, treated for 2-3 weeks in sphere culture

7. Effect of $24 \mathrm{~h}$ treatment with $10 \mu \mathrm{M}$ AS1411 on Rho ${ }^{\text {neg }}$ side population MCF7 cells

8. Effect of $24 \mathrm{~h}$ treatment with 5 or $10 \mu \mathrm{M}$ AS1411 on $\mathrm{ALDH}^{\text {br }}$ unsorted DU145 monolayer cells

9. Soft agar colony formation of DU145 cells

10. Sphere survival assay

166

11. In vivo tumor growth curves of DU145 cells pretreated with $10 \mu \mathrm{M}$ AS1411 for 72 hours 


\section{CHAPTER 1}

\section{INTRODUCTION TO NOVEL CONCEPTS IN ONCOLOGY RESEARCH: APTAMERS AND TUMOR INITIATING CELLS}

\section{BACKGROUND}

\section{Prostate cancer and the need for new therapies}

Cancer is responsible for one out of every four deaths and ranks as the second leading cause of death in the United States (1). More than 11 million people were estimated to be living with a history of cancer as of 2005 (the most recent statistic) $(\underline{2}, \underline{3})$. In 2008, The National Institute of Health estimated the cost of cancer to the nation at over $\$ 228$ billion for the year $(\underline{2}, \underline{3})$. Although the five year survival rate for cancers of all types has improved from $50 \%(1975-1977)$ to $66 \%(1996-2004)$, the persistently high impact of cancer on the American population, including patients, their families, and the economy, make further endeavors in cancer prevention, detection, and treatment a necessity in this country. In addition, according to the World Health Organization, cancer rates in the world are on the rise. Reasons underlying this statistic include a longer-lived - and thus increasingly aged - population and changes in lifestyle, such as Westernization of the diet and increased rates of smoking, in areas with previously low cancer incidence. It is clear that cancer is a serious problem that affects a large portion of the world's population and will continue to do so for years to come. 
Prostate cancer is the most common non-cutaneous cancer type diagnosed among men in the Western world. After lung cancer, it is the second leading cause of male cancer-related death in the United States (2). In 2010, approximately 217 thousand men were diagnosed with prostate cancer and 32 thousand died from this cancer (the approximate capacity of 68 jumbo jets). African American men are particularly vulnerable to prostate cancer with a death rate more than double that of white men (2). The reasons behind this discrepancy are incompletely understood at this time, but merit further investigation. The yearly cost of treating prostate cancer has been estimated at $\$ 2.3$ billion, making it the most expensive cancer to treat among men (followed by lung and then colorectal) (3). Prostate cancer patients who undergo androgen ablation generally experience initial regression of their disease which may last for several years. However, with time, most patients will go on to develop castration-resistant prostate cancer (CRPC) that must then be treated with chemotherapy. The current first line chemotherapy for such patients is largely ineffective, improving overall survival by only a few months $(\underline{4}, \underline{5})$. In the last year, four new drugs have been approved as second line chemotherapy for CRPC (one for palliation of bony metastases), but again the increase in survival is a few months at best (ㅁ). Additionally, chemotherapy drugs are generally poorly selective, causing serious side effects due to damage to non-cancer cells. The average age at diagnosis for men with prostate cancer is 70 years of age (2). Therefore, developing more specific therapies is especially important for geriatric patients who often cannot tolerate highly toxic regimens.

Although early stage prostate cancer typically has a high survival rate, there remains a clear need for better treatments. Surgical prostatectomy involves significant 
risk of major side effects like impotence and incontinence. These adversely impact quality of life, highlighting the need for new medical technologies that lessen the risk to patients. Moreover, advanced prostate cancer is generally insensitive to chemotherapy and has a particularly poor prognosis, so novel agents are sorely needed.

\section{Oligonucleotides as medicines}

Small pieces of synthetic nucleic acid (oligonucleotides or nucleic acid oligomers) are now commonly used in a number of different ways as tools for laboratory research. Interest is also growing in the possible applications of oligonucleotides as medically useful agents. Strategies utilizing oligonucleotides (both in the laboratory and in the clinic) are many:

- Antigene - a synthetic DNA oligomer targets a portion of the genomic double-helix DNA to form a triple helix and block transcription of that region $(\underline{7-9})$.

- Antisense - a DNA oligomer is designed to be complementary to mRNA encoding a specific protein. Base-pairing between the oligomer and the mRNA results in a double-stranded molecule which is degraded by the cell, blocking translation of the protein of interest $(\underline{10}, \underline{11})$.

- RNA interference - the oligomer is a double-stranded RNA that is processed intracellularly to single-stranded molecules. The RNA-induced silencing complex (RISC) pathway is activated, causing degradation of a specific endogenous mRNA species $(\underline{12})$.

- Transcription decoy - a double-stranded DNA oligomer mimics the target of a specific transcription factor, but does not result in production of protein. The decoy 
thus decreases the expression of the endogenous sequence (13).

- Immunostimulating - DNA oligomers containing the $\mathrm{CpG}$ (i.e. cytosine-guanine) motif act as agonists of Toll-like receptor 9 (TLR9), causing activation of dendritic cells and B cells $(\underline{14}, \underline{15})$.

- Aptamer - a single-stranded DNA or RNA oligomer folds into a sequence-determined three dimensional shape that binds specifically to a molecular target and (generally) inhibits its function $(\underline{16-20})$.

Though the field of nucleic acid oligomers as clinically useful medications has immense potential, the major hurdle of nucleic acid oligomer therapeutics of all types is whether or not the drug actually reaches its intended human cellular target. Optimally, any such therapy would be given in a systemic fashion and thus must have enough molecular stability to withstand degradation while traveling through the patient's circulation. Unfortunately, oligonucleotides by nature are particularly vulnerable to degradation by serum nucleases such that the half-life of most oligonucleotides after systemic administration is on the order of minutes. Another limitation of oligonucleotides with intracellular targets (including all those that act via RNA interference) is the low degree of intrinsic cellular uptake. Many bacteria utilize uptake of extrinsic DNA fragments as an important method to maintain genetic variability within the species, and have evolved cellular mechanisms for competency. In contrast, eukaryotic cells have protective mechanisms against competency, which in nature act to defend the cell against viral infection.

Several methods have been attempted to address these two major challenges (stability and uptake) in the clinical use of nucleic acid oligomers. One strategy is by 
enhancing resistance to degradation by modifying the nucleotides that make up the oligomer, such as substitution of an amine of fluorine group at the $2^{\prime}$ position of the ribose ring. The phosphodiester linkage of the oligomer backbone may also be modified by replacing a non-binding oxygen with a sulfur atom to form a phosphorothioate bond. While these changes do achieve the intended effect of increasing the molecular stability of the oligomer, they may have detrimental effects such as decreased activity or increased toxicity. In terms of the poor delivery of oligonucleotides to the intracellular compartment, researchers have employed various vehicles such as liposomes, polymer vesicles, genetically engineered viruses, and nanoparticles $(\underline{21-28})$. These methods work very well in vitro, and are invaluable tools in laboratories worldwide, but translation into practical clinical use is limited at this time.

In summary, although a number of methods designed to enhance oligomer stability and/or cellular uptake exist, they all generally decrease the ability of the molecule to act at its intended target and/or increase the toxicity of the agent.

\section{G-rich oligodeoxynucleotides and guanine quadruplexes}

It is well-established that oligodeoxynucleotides (ODNs) enriched in guanine bases (guanine-rich oligodeoxynucleotides, or GROs) have distinct properties. Via hydrogen bonding, the guanines facilitate the formation of folded three-dimensional structures, namely the G-quadruplex $(\underline{29})$. This structure is by nature highly stable, with a melting point well above physiologic temperatures and a high degree of intrinsic resistance to both serum and intracellular digestive enzymes that is much greater than typical single- or double-stranded oligomers (days vs. hours). In addition, GROs have 
been reported as having enhanced cellular uptake in a number of different settings, even without any of the chemical modifications or convoluted delivery systems that classic oligomer therapy requires $(\underline{18}, \underline{30})$. The specific mechanism behind the increased uptake of GROs is unclear despite decades of research, but may be at least in part secondary to the molecular stability of the higher order structure.

\section{Aptamers: a new class of cancer drug}

Of the different types of nucleic acid oligomer therapeutics, our laboratory is focused on the study of aptamers. Aptamer therapeutics are particularly pertinent to the field of medical oncology. Currently available chemotherapy drugs can be generally divided into two categories: small molecules or monoclonal antibodies. Small molecules are typically toxic chemicals, and were the first type of pharmaceutical used to treat cancer. Examples include cyclophosphamide, a nitrogen mustard, and cisplatin, a platinum derivative. Both these and other drugs nonspecifically kill cells by causing DNA damage, and like most small molecule therapeutics, have considerable toxicity to nonmalignant cells. This non-specificity is responsible for the classic chemotherapy side effects, including hair loss, gastrointestinal upset, and bone marrow suppression. Therapy is based on the rationale that cancer cells are more vulnerable to DNA damage than healthy cells.

Monoclonal antibodies are much larger than small molecules, being comprised of four large protein chains. Antibody therapy operates on the premise that tumor-specific antigens exist which can be used to distinguish cancer versus normal cells (e.g., trastuzumab, which binds the signaling molecule HER2/neu that is overexpressed in 
many breast cancers, or to target processes that are critically important for tumor growth (e.g. bevacizumab, an anti-VEGF antibody that inhibits angiogenesis). Antibodies are a much more recent addition to the chemotherapy pharmacopeia, with the first anticancer antibody approved in 1997 for the treatment of non-Hodgkin lymphoma (11). The major advantage of anticancer monoclonal antibodies is the high degree of specificity with which they bind their antigen target, making them as selective as the antigen against which they were raised.

Each of the two classes described thus far has its own intrinsic advantages and disadvantages in terms of clinical use. Aptamers, made of synthetic DNA or RNA that bind to specific targets, comprise an entirely new class of cancer drug. An aptamer's function lies beyond the traditional paradigm of DNA and RNA acting as passive blueprints for proteins or binding in a Watson-Crick sequence-dependent manner as required for RNA interference therapies. Instead, the basis for aptamer binding is the molecule's specific three-dimensional shape. Just as proteins have a specific structure determined primarily by the amino acid sequence, aptamers often contain secondary structure motifs (e.g. hairpins, G-quartets) determined by the sequence of their nucleotide bases. The mechanism of action for aptamers is, in essence, like that of monoclonal antibodies.

In terms of size, aptamers fall between traditional small molecules and large antibody proteins, and they have advantages of both of these classic drug types. Aptamers are specific for their molecular targets and less likely to cause toxic side effects than small molecules. Functionally, in terms of target-binding, aptamers are on par with monoclonal antibodies, yet aptamers have additional desirable characteristics including 
ease of manufacture, molecular stability (i.e. longer shelf life), and lower immunogenicity. In addition to frank therapeutic effects, aptamers may also be utilized as vectors for targeted drug delivery or for imaging. The same intrinsic characteristics that make aptamers appealing as pharmaceuticals also allow them to be easily employed for these ancillary purposes; attaching a highly specific aptamer to a drug or radionuclide that lacks cancer cell specificity would result in targeted delivery of the non-specific molecule. Aptamers lend themselves easily to these types of chemical modifications. Compared to most other nucleic acid therapies, aptamers do not necessarily require cellular uptake in order to function and need not be limited by transmembrane transport.

Aptamers are also being explored for non-oncologic purposes. Essentially any application that currently uses monoclonal antibodies could be performed with a less expensive, more stable, and similarly target-specific aptamer. This includes many research assays such as Western blotting, flow cytometry, immunohistochemistry, and ELISA. Several aptamers are also in different stages of human clinical trial, particularly for anticoagulation $(\underline{19}, \underline{20})$. The first aptamer to be approved for clinical use is pegaptanib, an intraorbital injection for the treatment of wet macular degeneration, which targets vascular endothelial growth factor (VEGF). It was approved by the Food and Drug Administration in 2004 (32).

\section{SELEX (systematic evolution of ligands by exponential enrichment)}

Aptamers may be engineered through a process called SELEX (Figure 1), an iterative procedure of approximately 10-20 cycles. The SELEX technology uses intrinsic binding affinity to isolate candidate aptamer sequences that bind to a target of interest 


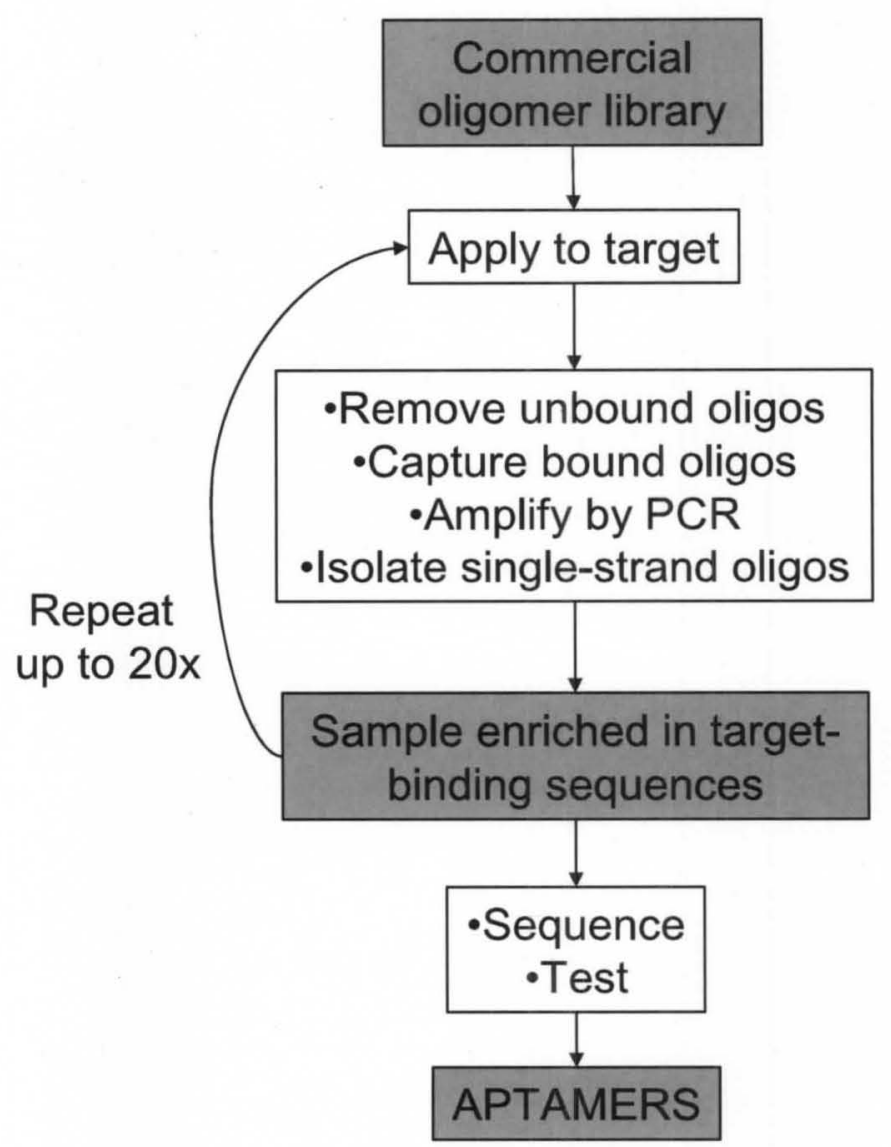

Figure 1: Schematic of SELEX workflow. An oligonucleotide library containing a mixed population of billions of sequences is applied to a specific target. Sequences that bind are retained and amplified by PCR. The new pool enriched in target-binding sequences is reapplied to the target, and the cycles repeated until aptamer sequences become prominent. 
(typically a purified protein) from an oligonucleotide library of millions of individual species. Briefly, a library of single-stranded DNA or RNA molecules of different random sequences flanked by known primer-binding regions is applied to the target. For a library of 26 unknown bases between the primer-binding regions, the number of possible different species is on the order of $10^{15}$ (26 positions, with each position having equal likelihood of being A, C, T, or G; $4^{26}=4.5 \times 10^{15}$ possible sequences (Chapter 2)). Some sequences will bind to the target. The unbound sequences are removed, and those remaining are detached and amplified by PCR. The PCR product, now enriched in targetbinding sequences, is purified and then reapplied to fresh target for the next round of SELEX. As these steps are repeated, the sequences that bind with greatest affinity will become increasingly prominent, and candidate aptamers can be identified.

Aptamers generated by SELEX have the same problems with molecular stability (i.e. resistance to degradation) and cellular uptake as other therapeutic nucleic acid oligomers. In addition to the previously discussed standard oligomer modifications to enhance stability, SELEX may address this issue "upstream" with the use of chemically modified or L-configuration nucleotide bases in the starting libraries and PCR reactions such that the selected aptamers have intrinsic stability and do not require downstream modifications that may adversely affect their activity or safety profile.

In this project, two upstream SELEX variations to improve molecular stability and cellular uptake are described and will be discussed fully in Chapter 4 . The first is the utilization of a starting oligomer library that is G-rich. We propose that such a library will contain fewer sequences, with a higher proportion of molecules that can form Gquadruplexes. The library will thus have more molecules with the naturally enhanced 
stability and uptake associated with this tertiary structure. This hypothesis is strengthened by the observation that many aptamers produced by SELEX using the standard (non-Grich) starting library have been reported to be able to form G-quadruplexes. Our second variation is to perform whole-cell SELEX; but, instead of simply selecting sequences that bind to the cells, we select for those that have been internalized by the cell. Rather than attempting to target a particular mechanism to transport the oligomer across the cell membrane, we propose to let the cell determine which sequences are able to do so. This method could handily address the issue of poor cellular uptake of oligomers, allowing us to generate efficiently internalized aptamers that would be ideal for drug delivery of molecules such as siRNAs for which transport across the cellular membrane is an effective-dose-limiting hurdle.

\section{"AS1411" is the first anticancer aptamer in human trials}

Dr. Paula J. Bates (the mentor for this proposal) and her colleagues have already discovered aptamers that selectively kill cancer cells (33-37). One of these, a G-rich oligodeoxynucleotide (ODN) called AS1411 (previously known as AGRO100), is the first anticancer aptamer to enter human clinical trials (18). AS1411 was initially discovered following a chance observation that a particular DNA sequence had significant antiproliferative activity against the DU145 prostate cancer cell line. Further studies on AS1411 proved that it binds to nucleolin, a protein that is highly expressed on the surface of cancer cells but not normal cells. Dr. Bates' work thus not only led to a new anticancer drug, but also a new class of anticancer molecules and a new anticancer target. 
Phase I and Phase II clinical trials of AS1411 have shown promising results that are remarkable for the lack of major side effects $(\underline{17}, \underline{38})$. AS1411 has been tested against metastatic renal cell carcinoma (MRCC) and acute myelocytic leukemia, and may ultimately prove useful in treating other metastatic cancers such as prostate. Of twelve MRCC patients in the Phase I trial, one had a complete response, one had a partial response (70\% tumor shrinkage) and seven more had disease stabilization ranging from 2 to 15 months. This aptamer clinical trial is the first of its type, indicating an almost wholly unexplored niche for new, more specific anticancer aptamers.

The clinical response to AS1411 is very unusual in that a single treatment may have no immediate effect, but over several months may cause complete tumor regression with no additional treatment. Due to this curious effect, one hypothesis of this project is that AS1411 and other aptamers may act by targeting cancer stem cells.

\section{Tissue stem cells, cancer stem cells, and tumor initiating cells}

Tissue stem cells (also known as "adult stem cells" or "somatic stem cells") are capable of both differentiation and long-term self-renewal. They are now believed to exist in most if not all organs of the human body, remaining quiescent until signaled to divide for tissue maintenance or repair. Though elusive, the definitive identification of tissue stem cells is slowly being pinpointed in many different systems such as the bone marrow, skin, gastrointestinal tract, bronchial epithelium, and prostate gland (⒐42). These stem cells are also responsible for common hyperproliferative disease states such as hyperkeratosis and benign prostatic hyperplasia (BPH). In the prostate, the malignant version of such over proliferation is prostate cancer. 
It has long been recognized that cancers have diversity within their cell populations (e.g. stromal, immune cells), but until recent years, the extreme diversity of the cancer cell population itself within a single malignancy has been largely overlooked. Many recent publications report a rare subgroup, dubbed cancer stem cells (CSCs), which appear to divide and differentiate to make a tumor just as benign stem cells do to make a healthy tissue (43-46) . CSCs were first detailed in the context of acute myelocytic leukemia (AML) $(\underline{47}, \underline{48})$. Cells were isolated from human patients based on cell surface marker profiles and injected into mice. These cells proliferated and repopulated in the mice into AML pathologically identical to the disease in the original human patients. Other cells without this surface marker profile were wholly unable to cause cancer in the mice. Since this first report, CSC populations have been characterized in breast, brain, prostate, ovary, colon, and other solid tumors. In these organs, it may be only this small population (generally between 0.01 and $5 \%$ of the total cancer cells) that is able to establish and perpetuate a tumor.

Current anticancer therapies focus on rapid shrinkage of tumor volume, and indeed this is the primary objective of most cancer research models. However, the majority of cancer patients who enter remission will eventually suffer a relapse of their disease. The concept of CSCs could explain this sad phenomenon. If CSCs comprise only a tiny percentage of the total tumor, divide slowly, are resistant to chemotherapy, and are responsible for the perpetuation of the tumor, then even those cancer therapies that cause dramatic clinical responses may leave the CSCs unscathed (49). The surviving CSCs would reestablish the disease in time. On the other hand, a therapy that selectively targets CSCs may initially appear to have no effect on tumor growth, but over time the tumor 
would shrink and finally disappear. (Figure 2) This is precisely the effect shown by AS1411. Furthermore, most of the morbidity and mortality associated with cancer is due to distant metastases. If only CSCs are able to form a metastasis, then anti-CSC therapies could potentially be used as prophylaxis for cancer spread.

The nascent field of CSC research is fraught with conflicting reports as the methodology slowly becomes established. One confounding element is the inconsistency of the very definition of "cancer stem cell." In 2006 the American Association of Cancer Research convened a CSC workshop to discuss guidelines for this rapidly growing area of study. At that time a CSC was defined as "a cell within a tumor that possess[es] the capacity to self-renew and to cause the heterogeneous lineages of cancer cells that comprise the tumor" (므). Despite this guideline, many researchers use the term CSC for subgroups of cancer cells that can reproducibly grow a tumor; the feature of self-renewal is often not addressed. For this situation, the term tumor initiating cell (TIC) is preferable, and this term will be used throughout this body of work.

Different research groups propose varying and overlapping properties for TICs: TICs alone are sufficient for the formation of cancer, there are different types of TICs which may have varying tumor-forming capacities, TICs might make up the bulk of the tumor rather than comprising a rare subpopulation, and many cells (both benign and malignant) have to potential to become a TIC. Whether or not a true self-renewing CSC compartment exists, within a given malignancy there is a high degree of cellular heterogeneity that has been largely ignored in the development of new cancer therapies. Certain subpopulations of cells are known to be markedly more invasive, proliferative, and/or stem-like (i.e. having stem cell markers and features). Therefore, despite the 


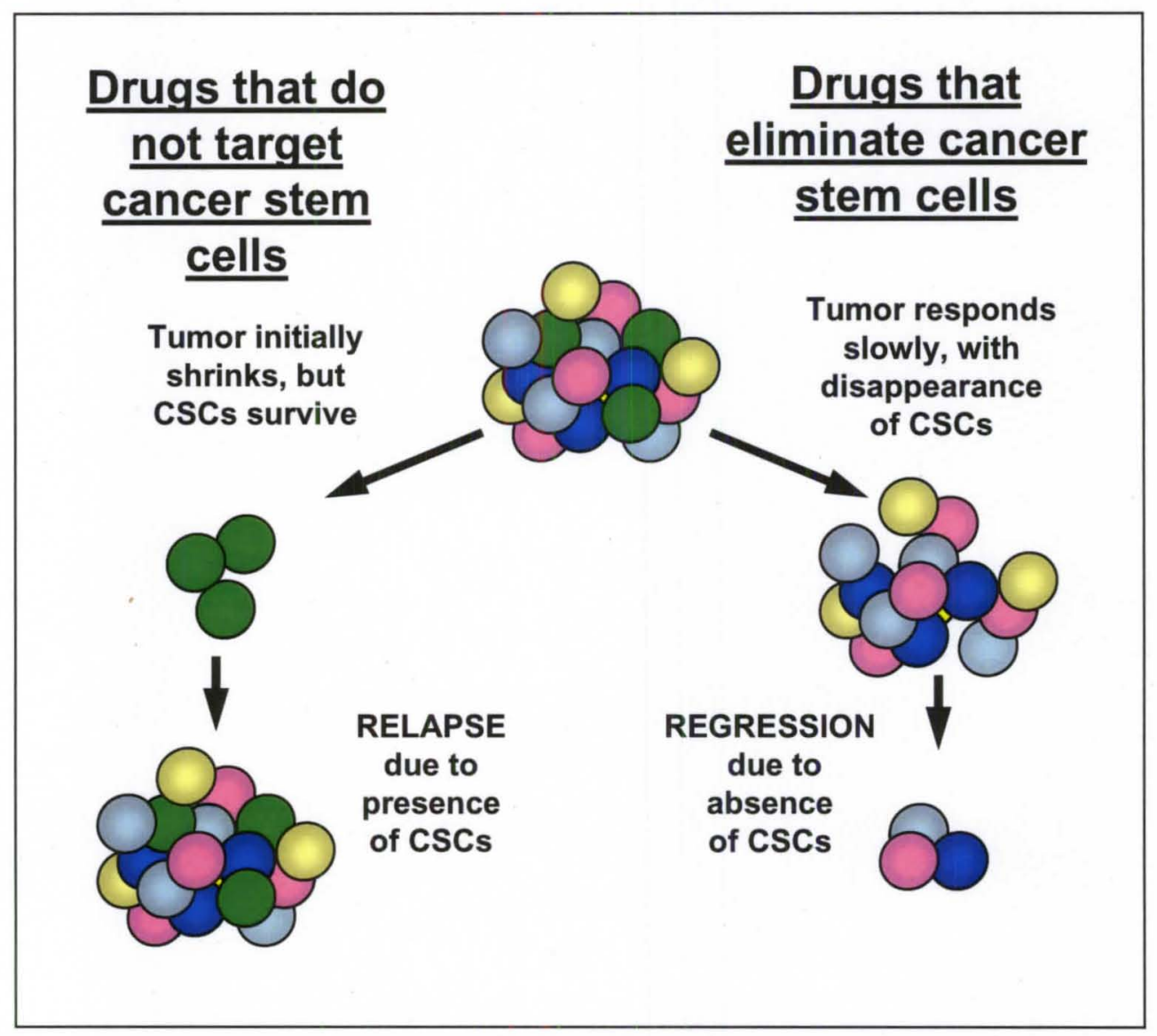

Figure 2: Cancer stem cells (green) and clinical implications. Treatments that spare cancer stem cells may initially have a dramatic clinical response, but given time the intact stem cell compartment will repopulate the tumor. Conversely, treatments that destroy the cancer stem cell subpopulation may appear to have limited initial effect, but with time the tumor spontaneously regresses. 
controversy surrounding the study and terminology of CSCs, TICs, and different cancer cell subtypes, the basic concept of a subpopulation of cancer cells that is particularly responsible for tumorigenicity is becoming widely accepted and must be addressed in cancer drug development.

\section{Aptamers and cancer stem cells}

Upon further consideration, aptamers and CSCs have many additional aligning features that make the study of their relationship even more compelling:

- CSCs are largely quiescent, making them less vulnerable to chemotherapies that target rapidly proliferating cells $(\underline{51-53})$. Unlike most chemotherapy drugs, aptamers do not require cycling cells for activity.

- CSCs have been shown to express multi-drug resistance efflux pumps that nonspecifically remove small molecules from the cell $(\underline{52}, \underline{54}, \underline{55})$. Aptamers are not small molecules and would likely be unaffected by these pumps.

- CSCs may have highly activated DNA damage responses, making ionizing radiation therapy and many anticancer drugs less effective $(\underline{43}, \underline{52})$. Aptamers do not appear to damage DNA as part of their mechanism of action.

- CSCs may prove to be difficult to access, either embedded deep within a tumor or within a protected stem cell "niche" $(\underline{43}, \underline{56})$. Aptamers have better tissue penetration compared to monoclonal antibodies, and may be able physically to reach CSCs more readily $(\underline{57}, \underline{58})$.

RATIONALE AND PURPOSE 
We believe the field of anticancer aptamers is worthy of further investigation. The initial intention of this body of work was the isolation of new cancer-specific aptamer sequences via whole-cell SELEX for potential use in clinical oncology. AS1411 was discovered by a chance observation. It is therefore likely that a deliberate approach to find new aptamers for specific cancer types will lead to even more promising anticancer molecules that may be used for drug-delivery, imaging, and even veritable pharmaceuticals.

We hypothesized that G-rich aptamers are likely to form G-quaduplexes, and may be particularly appropriate due to naturally enhanced molecular stability and ability to enter cells. We planned to test the utility of a G-rich, non-classical library (specifically, comprised of only $\mathrm{G}$ and $\mathrm{T}$ bases) compared with the classic random $\mathrm{N}$ library as the starting point for SELEX. To better understand the intrinsic characteristics of G-rich libraries in general, we studied the anticancer activity of a number of G-rich libraries in comparison with non-G-rich libraries. The unexpected observation that robust anticancer activity was exhibited by certain G-rich libraries, without the isolation of a unique aptamer sequence, led us to explore biological and biophysical characteristics and attempt to relate them to this intrinsic activity.

With respect to cancer stem cells, elimination of CSCs and TICs may prove to be the key to curing cancer and aptamers may be an effective way to target them. A second project goal of studying the ability of a G-rich aptamer to target cancer stem cells arose from observation of the clinical effects of AS1411. Though high risk, a positive result indicating that AS1411 is able to selectively target and destroy or neutralize CSCs would contribute greatly to the scientific bodies of knowledge of both aptamers and CSCs. 
Thus, this hypothesis-driven project was planned to explore the potential interactions between a G-rich aptamer and CSC/TICs, and possibly elucidate a key ability of a known G-quaduplex-forming anticancer molecule. To our knowledge, the project described here is the first to investigate the relationship between aptamers, a new class of cancer drug, and cancer stem cells, a revolutionary new cancer theory.

\section{HYPOTHESIS AND OBJECTIVE}

- Our hypothesis is that guanine-rich oligonucleotides are optimal for the development of new aptamers for use in the treatment of cancer, and may have particular utility in the treatment of cancer stem cells.

- Our objective is to develop new targeted aptamers specific for the treatment of prostate cancer.

\section{SPECIFIC AIMS}

Aim 1: Study guanine-rich oligodeoxynucleotide libraries for use in whole cell-SELEX.

Aim 2: Use non-conventional (G-rich) oligodeoxynucleotide library to identify aptamer candidates specific for prostate cancer cells and characterize selected aptamer candidates.

Aim 3: Test the hypothesis that a model aptamer (AS1411) targets CSC/TICs. 


\section{CHAPTER 2}

\section{CANCER-SELECTIVE ANTIPROLIFERATIVE ACTIVITY IS A GENERAL PROPERTY OF SOME G-RICH OLIGODEOXYNUCLEOTIDES}

\section{INTRODUCTION}

The appended manuscript describes the characterization of general properties of G-rich and non-G-rich libraries of single-stranded oligodeoxynucleotides (ODNs). This report has been published in the peer-reviewed journal Nucleic Acids Research (풍 Appendix A). As discussed, the underlying hypothesis of this body of work is that G-rich ODNs are particularly suited for development as new medical therapies due to naturally enhanced molecular stability and cellular uptake. We performed a number of experiments to establish baseline characteristics of G-rich versus non-G-rich ODN libraries, including antiproliferative effects upon a variety of cell lines. We did this in preparation of the SELEX project described in Chapter 4, in which we attempt the generation of cancerspecific aptamers from a non-traditional, G-rich ODN library. The purpose of these experiments was initially to test our hypothesis that G-rich libraries would be enriched in G-quadruplex forming sequences, which would have increased nuclease resistance and cellular uptake compared to traditional libraries.

Briefly, eleven libraries and three monobasic ODNs of varying lengths and nucleotide compositions were studied. We originally anticipated a lack of antiproliferative activity (as well as lack of molecular stability and cellular uptake) in the 
libraries, whereas after isolating candidate aptamer sequences via SELEX we expected to demonstrate an enhancement in activity. Although we postulated that G-rich libraries would contain a higher proportion of biologically active molecules, we presumed that the effects of any such molecules would be masked by the remaining millions of different sequences that comprise even a smaller, sequence-constrained library. On the contrary, however, we observed that G-rich libraries comprised of $\mathrm{G}+\mathrm{T}$ or $\mathrm{G}+\mathrm{C}$ oligodeoxynucleotides exhibited robust inhibition of cancer cell growth while having little to no effect on the non-cancerous fibroblast cell line. Interestingly, the library comprised of $\mathrm{G}+\mathrm{A}$ bases did not have this effect. This manuscript is the first to report cancer-specific antiproliferative activity exhibited by a library of ODNs rather than a single, carefully isolated sequence.

Based on these unexpected results, a complete scientific project evolved. Our experimental ODN samples were divided into those that inhibited the growth of cancer calls and those that did not. The two groups were then compared for biophysical and biological characteristics, namely higher order structure, molecular stability, cellular uptake, and protein binding, in an attempt to correlate these features with this cancerspecific activity.

\section{CONCLUSIONS}

In this manuscript, we conclude that cancer-selective antiproliferative activity requires "nuclease-resistance, efficient cellular uptake, and binding to specific protein(s)." Though we observed some exceptions, as a general rule we showed that the libraries that have these biological and biophysical characteristics appear to form G- 
quadruplexes. It is noteworthy that, although G-quadruplex formation appears to be a prerequisite for cancer-selective antiproliferative activity, quadruplex formation alone is insufficient for this activity. This conclusion has also been reported by previous

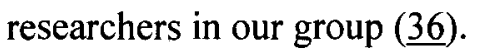

Our results, as well as any other elucidation of features that confer anticancer activity may aid in the isolation of new cancer-targeting aptamers via the SELEX procedure. For example, the identification of a sequence motif (e.g. GGTGGT) that correlates with cancer-selectivity if found specifically at the 3 ' end suggests the utility of a starting library in which every sequence ends with this motif. The starting SELEX library would be enriched in potential anticancer molecules from the outset, and the SELEX evolution process would select for target specificity.

Additionally, any constraint on the sequence or composition of an ODN pool of a given length yields a library that contains fewer molecules. Minimizing the length of the starting library while maintaining optimal biological activity would also reduce the number of molecules. A library that contains fewer sequences may translate into a decrease in the number of cycles required before candidate aptamer sequences become prominent enough to be identified, with potential corresponding effects on the costs of discovery and manufacture. For these reasons we studied both nucleotide composition and library length, and observed that a library with as few as eighteen nucleotide bases in length exhibited virtually the same inhibition of cancer cell growth as one nearly twice as long (34 bases).

We concluded that, in general, the "active" anticancer libraries showed evidence of G-quadruplex formation and that this feature was critical to the intrinsic activity of the 
library. We thus pursued the possibility of using a G-rich ODN library, smaller and enriched in G-quadruplex forming sequences, as the starting point for SELEX rather than the classic, larger, random $\mathrm{N}$ library (Chapter 4).

Regarding the GA 26mer ODN library, we observed that this library had a unique $\mathrm{CD}$ spectrum that is consistent with data previously reported by other researchers for G+A ODNs. The spectrum is believed to signify stacked guanine bases, but whether or not this translates into a G-quadruplex is uncertain at this time. It has been reported that a GA aptamer that binds to the bovine prion protein does form a G-quadruplex $(\underline{60}, \underline{61})$ but perhaps the majority of species within the GA $26 \mathrm{mer}$ library are unable to form this structure due to the relatively bulkiness of the adenine base compared to the pyrimidines (thymine and cytosine). If it is indeed formation of G-quadruplexes that is ultimately responsible for selective antiproliferative activity against cancer cells, it is likely that the percentage of G-quadruplex-forming sequences within the library determines the net presence or lack of antiproliferative activity of the library en masse. Therefore, if fewer quadruplexes are present in the GA library, it follows that the library would not exhibit this activity, along with the corresponding lack of the characteristic G-quadruplex protein binding and cellular uptake. This does not rule out the possibility of potential GAanticancer aptamers. However, regarding SELEX, we conclude that a GA starting library offers little benefit over the classic $\mathrm{N}$ library.

In our EMSA data, we observed unique protein bands in the GC 26mer and C 26 mer samples. The identification of the protein targets of the GC 26 mer library may be particularly significant in the development of new aptamers as cancer therapeutics, as this library had a unique profile of antiproliferative activity against the various cancer cell 
lines (Appendix A, manuscript, Figure 3E). The C 26mer did not exhibit cancer-selective antiproliferative activity, but had a unique $\mathrm{CD}$ spectrum suggestive of the i-motif tertiary structure (Appendix A, manuscript, Figure 4B) in addition to its unique protein band. Studies that identify GC- and/or and C-binding proteins would therefore be a contribution to the body of knowledge of aptamers and ODN therapies.

In the following chapter (Chapter 3), we discuss GC and C ODNs in greater detail. We describe additional experiments that we performed to identify proteins from cancer cell extracts that bind to the GC $26 \mathrm{mer}$ library and the C 26mer ODN. We discuss the implications of the identified proteins, including possible mechanisms of action. We close Chapter 3 with a description of future experiments and directions, especially the validation of proteins identified as binding to these ODNs of particular interest. 


\section{CHAPTER 3}

\section{PROTEIN TARGETS OF THE GC OLIGODEOXYNUCLEOTIDE 26MER LIBRARY AND POLY-DEOXYCYTOSINE 26MER MOLECULE}

\section{INTRODUCTION}

\section{The significance of nucleic acid-binding molecules}

It was at one time accepted that the role of nucleic acid within a cell was largely passive. According to the central dogma of molecular biology, DNA acts as an information repository that is passed down during cell division and contains instructions to make proteins. Proteins were thought to be the active components of biology that perform virtually all essential cellular functions. RNA was believed to be little more than the machinery that translates the DNA information into the final functional protein end product.

While the central dogma still holds true, it is now known that the nucleic acids of the cellular genome play a more nuanced and dynamic role that first envisioned. DNA in and of itself has intrinsic functional activity including regulation of gene expression and intracellular signaling. Epigenetic modifications have been implicated in a number of disease states including Alzheimer's disease, rheumatoid arthritis, and several kinds of cancer (62-64). RNA comes in a variety of subspecies with different functions such as riboswitches, ribozymes, and microRNAs, in addition to the classic mRNA, tRNA, and rRNA types $(\underline{65}, \underline{66})$. Clearly, nucleic acids are both more complex and much more 
biologically active than was once postulated, and will require further investigation in order to be fully appreciated. It follows that identifying and elucidating molecules that bind to nucleic acids, including both endogenous and synthetic aptamer species, will have important implications not only in the development of medical therapeutics (the goal of this project), but also in fundamental molecular biology.

Treatment strategies in the development of new pharmaceutical agents that utilize nucleic acid binding may thus be designed in two different ways. The first is by engineering synthetic nucleic acid aptamer therapeutics to bind to clinically, relevant targets. The second is by identifying endogenous nucleic acid structures that play important roles in disease states and designing small molecules, or monoclonal antibodies, or even nucleic acid aptamers that target them.

\section{Rationale for studying GC ODN library- and dC-binding proteins}

Our manuscript (Appendix A) makes the following observation regarding protein binding using radiolabeled ODN libraries and monobasic samples and a whole cell lysate from prostate cancer cells: that the GC 26 mer library (which has intrinsic and selective antiproliferative activity against cancer cells) and the C 26 mer ODN $\left((\mathrm{dC})_{26}\right.$, which does not have antiproliferative activity at all) each exhibit unique protein bands that differ both from one another and from all the other libraries and monobasic ODNs that we studied (Appendix A, Figure 7, B3 and B4, respectively).

These unique protein binding data are of particular scientific interest for the GC library, as this library has different cancer type selectivity than the GT libraries (Appendix A, Figure 3A); while the GT libraries inhibited the growth of every cancer cell 
line tested, the GC library exhibited antiproliferative activity similar to the GT libraries against the lung and prostate cancer cell lines, but only moderate activity against breast cancer cells. Correspondingly, the fluorescently-labeled GC 26mer library exhibited cellular uptake that was comparable to the GT library in lung and prostate cancer cells, but relatively reduced in breast cancer cells. It may be that the GT and GC libraries act by distinct mechanisms of action and through distinct molecular targets, and that the target of the GC library is expressed at different levels in the breast cancer cells compared to the other cancer cell types tested. Identification of such a target would shed light upon a possible tumor specific target as well as the mechanism of the cancer-selective antiproliferative effect of the GC ODN library. Such data would also suggest the possibility of using an ODN library containing only guanine and cytosine as a starting point for SELEX to generate aptamers against specific cancer types known to be enriched in that target molecule. As discussed in Chapter 1, we hypothesize that guanine-rich oligonucleotides are optimal for the development of new aptamers for the treatment of cancer and one of our specific aims is to study guanine-rich ODN libraries for use in SELEX against cancer cells. The study of proteins that bind to the GC library therefore furthers the goals of our project.

The primary aim of this body of work is to develop new aptamers for use as oncology therapeutics. Although the poly-C ODN exhibited no intrinsic anticancer activity, cytosine-rich stretches of DNA are able to form a highly dynamic, pH-dependent quadruplex structure called the "i-motif" (discussed further below). This non-canonical nucleic acid structure may play a regulatory role by acting as a biological switch in the activity and/or expression of guanine-rich areas, as a cytosine-rich strand is the natural 
complement of such regions. Guanine-rich regions (that may themselves form Gquadruplexes) are prevalent throughout the human genome, and include centromeres, chromosome 19, immunoglobulin switch regions, and disease states involving the $\mathrm{X}$ chromosome (Fragile X) $(\underline{67}, \underline{68})$. G-rich regions are also seen in the promoters of many genes important in cancer biology, such as c-myc, c-kit, VEGF, HIF-1, KRAS, MEN-1, RET, and RB ( $\underline{69})$. Perhaps the most well-known G-rich area of the human genome is the telomere, which has far-reaching implications and effects on the biologies of cancer and of aging, and is an attractive therapeutic target of much pharmaceutical interest. Several citations in the literature report that the corresponding $\mathrm{C}$-rich telomere strand forms $\mathrm{i}$ motifs that may eventually be shown to act in the regulation of the telomere, and thereby impact in the field of oncology $(\underline{70-72})$.

Only a handful of proteins that specifically bind to poly-C ODNs and/or the imotif have been identified ( $\underline{70})$. Therefore, the identification of a new i-motif-binding protein, though perhaps not overtly pertinent to the development of new anticancer aptamers, would be a contribution to the scientific field of aptamers and their targets and could eventually be significant in the development of cancer therapeutics.

We chose not to characterize proteins binding to the GT library because we suspected that such proteins would have a high degree of redundancy with the proteomic analysis of AS1411, ODN comprised solely of guanine and thymine bases that has been extensively characterized by our laboratory previously (구). As expected, each library exhibited a high number of protein bands; we focused our efforts only on the bands in the GC and C 26mer samples that exhibited distinctive features and were likely to provide novel information. 


\section{Guanine-cytosine ODNs}

Endogenously, stretches of nucleic acid that are especially GC-poor or GC-rich have been reported to have important biologic functions. The guanine-cytosine base pair is joined by three hydrogen bonds, while the adenine-thymine bond is joined by only two. Therefore, sections of genomic DNA that require frequent separation, such as promoter regions, tend to have low GC content. Conversely, nucleic acids with high $\mathrm{GC}$ content may form higher order structures that depend on this strong complementary base pairing. For example, structured RNA species (e.g. tRNAs, rRNAs, ribozymes) often contain a high percentage of $\mathrm{G}+\mathrm{C}$ bases which incur molecular stability to the fragile RNA molecule by forming shapes such as stem-loops and cloverleaves (3). Riboswitches are a type of structured RNA that are common in bacterial species, and denote an untranslated portion of an mRNA molecule that may bind to proteins that regulate expression of that mRNA - in other words, an endogenous RNA aptamer. One recent report describes a new class of riboswitch that binds to the biologically important molecule S-adenosylmethionine which was identified by analyzing regions of genomic DNA with relatively high GC content that did not contain known genes, but did precede metabolic genes. The authors' rationale was that stretches of DNA with these features were likely to be enriched in such metabolically active RNA species (4)).

Another role of GC-rich stretches of DNA involves epigenetic modifications. Epigenetic methylation occurs most often upon cytosine bases that are immediately followed by a guanine ( $\mathrm{CpG})$. The term "CpG island" is defined as a region that 1$)$ is greater than or equal to 200 bases, 2) is comprised of at least $50 \%$ cytosines and guanines (i.e. $\mathrm{GC}$-rich), and 3) has an observed to expected $\mathrm{G}+\mathrm{C}$ ratio of at least 0.6. Given this 
definition, $\mathrm{CpG}$ islands are found in the 5 , promoter region of approximately $60 \%$ of human coding genes, and methylation at or around the $\mathrm{CpG}$ islands can block transcription of those genes. Aberrant methylation of promoters has been implicated in a number of disease states; global hypomethylation is a known feature of cancerous cells, as is hypermethylation of important tumor suppressor genes (2). The study of GC-rich nucleic acids may therefore impact the study of oncology.

In addition to sequences containing approximately equal numbers of Gs and Cs, individual molecules within the GC library may contain either relatively more guanines or relatively more cytosines. Guanine-rich molecules may form the highly stable Gquadruplex, while cytosine-rich molecules may form the i-motif. One must also consider the possibility that multiple molecules within the library may assemble into a multiplex, forming not only secondary and tertiary, but also quaternary structures with or without complementary base pairing. It may be that the GC library exhibits its distinct profile of antiproliferative activity and unique protein binding pattern due to the prevalence of all three structural features: GC-base pairing, G-quaduplex, and i-motif, and that the structures may be formed by one or more individual ODN molecules.

\section{Poly-dC ODN and the i-motif}

Although C-rich stretches of nucleic acid are relatively less well-studied than many other nucleic acid motifs, there exists a small, highly conserved family of molecules known as poly-C binding proteins (PCBPs) that play a role in posttranslational regulation as well as acting as transcription factors. Certain PCBPs have been shown to act in the transcription and posttranscriptional regulation of the androgen receptor, an 
important signaling pathway in prostate cancer $(\underline{75}, \underline{76})$. An increase in PCBP1 levels was associated with a decrease in AR expression, and knockdown of PCBP1 caused an increase in AR. As AR is not expressed in prostate cancer stem-like cells, PCBPs may be a potential therapeutic target. The C-rich telomere strand is also known to bind PCBPs.

In addition to the study of PCBPs and their targets, there is a growing field of study concerning the "i-motif," a higher-order nucleic acid structure based upon the binding of cytosine residues. The i-motif was first described as "two parallel duplexes (antiparallel to one another) with...intercalating base pairs," i.e., the i-motif is a cytosine based four-stranded tertiary nucleic acid structure $(\underline{77})$. The i-motif has been reported with both DNA and RNA species, though the RNA structure is much less stable (ㅍ).

The i-motif is $\mathrm{pH}$ dependent, and is widely believed to require slightly acidic conditions in order to be formed. However, in our manuscript (Appendix A) we demonstrate a series of $\mathrm{CD}$ spectra of the $\mathrm{C} 26 \mathrm{mer} \mathrm{ODN}$ at a range of $\mathrm{pH}$ that suggest the formation of the i-motif up to a $\mathrm{pH}$ of 7 ; other researchers report similar observations at neutral and even somewhat alkaline $\mathrm{pH}(\underline{59}, \underline{79})$. If the i-motif is able to be formed at physiologic $\mathrm{pH}$, then it is likely to be biologically relevant. Alternatively, the i-motif is known to be highly dynamic and readily affected by the nucleic acid's microenvironment ( $\mathrm{pH}$, ion concentration) (2009, Zhou et al.), and therefore could function as a regulatory switch. Manipulation of the i-motif formation and stability could then be used to exploit biologic function. As an example, single-walled carbon nanotubes have been shown to induce the formation of the $\mathrm{i}$-motif in telomeric DNA and to stabilize this structure, enabling the S1 nuclease to cleave the single-stranded hairpin loops resulting in degradation of the telomere $(\underline{80})$. This phenomenon may potentially be exploited for 
therapeutic purposes in the investigation of cancer and also of aging.

\section{Identification of GC- and C-ODN-binding proteins}

After submission of our manuscript describing cancer-selective antiproliferative activity of certain guanine-rich ODN libraries, we subsequently pursued the identification of proteins that bind to the GC and C ODNs. We studied these ODNs in comparison with the $\mathrm{N}$ and GT libraries, reasoning that the unique bands seen in the EMSA data (Appendix A, Figure 7) should be present in samples containing cellular lysate and GC library or poly-dC ODN, and absent in the $\mathrm{N}$ and GT libraries. The methods and results from this preliminary experiment identifying GC- and C-ODN-binding proteins are included here.

\section{METHODS}

\section{Pull down of GC 26mer- and C 26mer-binding proteins}

Three libraries and one monobasic ODN were ordered with biotin attached to the 5 ' end: N 26mer, GT 26mer, GC 26mer, and C 26mer. Whole cell lysate of DU145 cells was prepared using RIPA buffer (50 mM Tris- $\mathrm{HCl}(\mathrm{pH} 8.0), 150 \mathrm{mM} \mathrm{NaCl}, 0.02 \%$ sodium azide, $1 \% \mathrm{NP}-40,0.5 \%$ sodium deoxychol, $0.5 \mathrm{mM}$ DTT, $1 \mu \mathrm{g} / \mathrm{mL}$ aprotinin,) containing $2 \mathrm{mM}$ PMSF and $1 \mathrm{x}$ protease inhibitor cocktail (Sigma, St. Louis). One $\mathrm{mg}$ of cell lysate was preincubated with $10 \mu \mathrm{M}$ biotinylated ODN, then incubated with Neutravidin (a streptavidin analogue) agarose resin (Thermo Scientific). Beads containing bound ODN-protein complexes were washed thrice. Bound proteins were eluted in 2x SDS-PAGE loading buffer containing 5\% $\beta$-mercaptoethanol. 


\section{Separation of pull down samples}

Samples were subjected to SDS-PAGE using an $8 \%$ polyacrylamide gel. The gel was washed thrice in water, stained with Simply Blue Safe Stain (Invitrogen) for one hour, and destained in water overnight at room temperature. The binding, elution, and SDS-PAGE was repeated once, and the samples in the second gel allowed to electrophorese for a longer period of time in order to achieve better resolution of the higher molecular weight proteins.

\section{Mass spectroscopy analysis of unique protein bands}

Candidate bands from the GC 26mer and C 26mer lanes (e.g. uniquely present in these samples versus N 26mer and GT 26mer) were excised and digested using a commercial trypsin-based kit (Trypsin Profile IGD Kit, Sigma). Samples were sent for mass spectroscopy analysis at the University of Louisville core facility.

\section{RESULTS}

\section{GC library and C 26mer bind unique proteins}

Based on the EMSA data, we used biotinylated ODNs (GC 26mer library and poly-dC monobasic $26 \mathrm{mer}$ ) to pull down proteins from DU145 whole cell lysate. Proteins were eluted from streptavidin beads, run on acrylamide gels, and stained with Coomassie blue (Figure 1). We then examined the gel for bands unique to either the GC $26 \mathrm{mer}$ sample or the $C 26$ mer sample. Though complicated by the presence of a high number of bands in the GT library (comparable to the pattern seen with AS1411), we identified a 


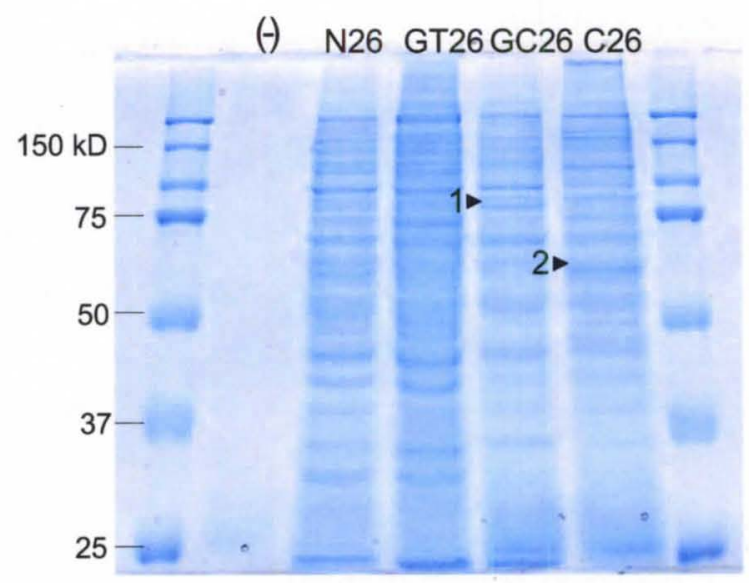

1. ATP-dependent DNA helicase 2 , subunit 2

2. Isoform 2 of heterogeneous nuclear ribonucleoprotein $\mathrm{K}$

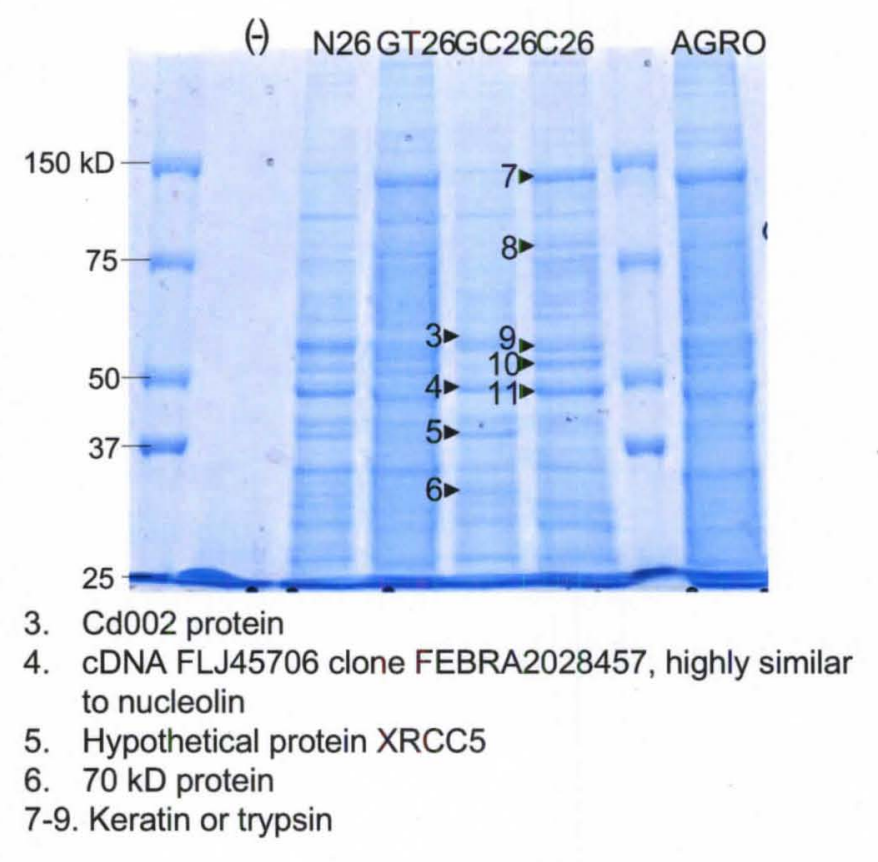

10. Isoform 2 of ATP binding cassette family F member 1

11. Isoform 2 of nucleolin

Figure 1: Precipitation of proteins from DU145 whole cell lysate with biotinylated oligodeoxynucleotide librares. ODNs with bound proteins were separated using agarose beads with a streptavidin analog. Excised proteins marked with arrowheads. Identified proteins as listed. (-) no library, N 26mer, GT 26mer, GC 26mer, and C 26mer monobasic ODN. 
number of unique bands in each sample which were excised. Upon analysis by mass spectroscopy, identifications were made for all eleven bands. The significant identifications are discussed below:

\section{GC library-binding proteins:}

ATP-dependent DNA helicase 2 subunit 2 (Ku80), IPI00220834.8

Hypothetical protein XRCC5 (Ku80), IPI00791570.1

$70 \mathrm{kDa}$ protein $(\mathrm{Ku} 70)$, IPI00465430.5

Cd002 protein, IPI00216746.1

cDNA FLJ45706 fis, clone FEBRA2028457 (highly similar to nucleolin), IPI00444262.3

\section{$\underline{\mathrm{Ku} 80 \text { and } \mathrm{Ku} 70}$}

These proteins are the two subunits that form the heterodimer eukaryotic DNA repair protein $\mathrm{Ku}$. $\mathrm{Ku}$ was originally discovered in the context of autoimmune disease, and notably is involved in the genetic recombination responsible for the development of the humoral immune system and the vast variety of antibody species (11).

Ku plays a critical role in double-strand break repair, both homologous repair and non-homologous end joining. It acts by binding to the free ends of linear, duplex DNA, and physically juxtaposing the two ends. Ku may then recruit other proteins into an active complex called DNA-dependent protein kinase (DNA-PK) to perform the necessary repair. The activity of the DNA-PK complex is regulated by the Ku protein. Ku therefore has wide ranging effects on cell proliferation, apoptosis versus tumorigenesis, 
chromosomal stability, and telomere maintenance $(\underline{82}, \underline{83})$. Mutations in either Ku80 or Ku70 have been implicated in a variety of cancer types, including gastrointestinal and hematologic.

In addition to their function as the $\mathrm{Ku}$ heterodimer, the subunits $\mathrm{Ku} 80$ and $\mathrm{Ku} 70$ may act as unique entities with their own specific functions. Data suggest that Ku80 and Ku70 may be active at many different subcellular locations. Though primarily nuclear, both subunits have been shown to translocate into the cytoplasm and to the cell surface. The impetus, regulation, and significance of these translocations are poorly elucidated at this time.

Though $\mathrm{Ku}$ has not been shown to bind to particular sequences of nucleic acid, it is known to bind to free ends of duplex DNA including blunt ends, ends with overhangs, hairpins, and telomeres (which contain a G-rich motif and a correspondingly C-rich complementary strand) ( $\underline{83})$. Because guanines and cytosines are natural complements, the GC 26 mer library likely contains molecules that either self-contain or assemble to form duplex DNA structures. It is therefore logical and exciting that molecules within our GC library bind to the Ku subunit proteins.

If compromised in either activity or expression, lack of $\mathrm{Ku}$ may lead to tumorigenesis due to its role in DNA repair. Conversely, if overactive, excessive DNA repair/end-joining may translate into resistance to chemotherapies. Ku could potentially be manipulated as therapeutic target, and aptamers evolved from a GC ODN library may be a good way to target this fundamentally important protein complex.

\section{$\underline{\mathrm{Cd} 002}$ protein}


This ubiquitously expressed human protein has been identified in a number of different contexts. Cd002 is officially known as endoplasmic reticulum-golgi intermediate compartment (ERGIC)-2, but is also known as Erv41, PTX1, and CDA14. The specific function of this protein is unknown.

As ERGIC2, this protein is reported to play a role in intracellular transport between the endoplasmic reticulum and the Golgi apparatus. The yeast homologue is called Erv41. As PTX1, this protein was first identified in 2001 via subtractive hybridization, i.e. expressed in normal prostate tissue but not in prostate cancer tissue. (84) Cd002 has been shown to be upregulated in nasopharyngeal carcinoma and downregulated in prostate cancer cells $(\underline{84}, \underline{85})$. PTX1 is located on chromosome 12 , a common site for mutations in advanced prostate cancer. When expressed in a prostate cancer cell line, PTX1 induced senescence. When downregulated in the same cell line, the cells exhibited increased tumorigenicity and invasiveness compared to control prostate cancer cells $(\underline{86})$. As CDA14, this protein was shown to have similar effects on a different prostate cancer cell line as well as being downregulated in three liver cancer cell lines, and to interact with human EF-1 $\alpha(\underline{87})$.

Nucleolin

Nucleolin was first described in 1973. It is a large protein that is ubiquitously and abundantly expressed with a diverse array of cellular functions. It is intimately involved in the formation of nucleolus, but is also found in the nucleoplasm, cytoplasm and on the cell surface. Nucleolin is purported to play myriad roles in RNA transcription, rRNA folding and ribosome formation, intracellular transport of molecules, mRNA stabilization, 
calcium homeostasis, cell adhesion, and response to viral infections. Nucleolin may be heavily modified with phosphate and methyl groups in response to different extracellular signals, and as protein synthesis is a critical element in cell growth, nucleolin acts as part of the pathways responsible for cellular proliferation. Nucleolin is also known to bind directly to oncologically important proteins such as c-myc, VEGF, and topoisomerase $(\underline{88-90})$.

As one may expect given nucleolin's role in different aspects of RNA function, nucleolin is a known generic nucleic acid binding protein. Among other nucleic acid species (including RNA) to which it binds, nucleolin has been shown specifically to bind guanine-rich oligonucleotides and G-quadruplexes, including the telomerase sequence and ribosomal genes $(\underline{36})$. We believe nucleolin is involved in the mechanism of action of AS1411, the G-rich aptamer discovered by Dr. Bates et al. that has been studied as a potential therapy for patients with acute myelocytic leukemia. Previous reports from our laboratory show that AS1411 binds to nucleolin, and that antiproliferative effect of AS1411 on cancer cells is correlated with the formation of a protein complex containing nucleolin, and that inhibiting nucleolin expression by siRNAs can inhibit certain biological activities of AS1411 (1ㅣ, $\underline{92})$.

\section{26mer-binding proteins:}

- Isoform 2 of heterogeneous nuclear ribonucleoprotein K (hnRNP K), IPI00216746.1

- Isoform 2 of ATP-binding cassette sub-family F member 1, IPI00013495.1

- Isoform 2 of nucleolin, IPI00827674.1 


\section{hnRNP K}

Heterogenous nuclear ribonucleoprotein $\mathrm{K}$ was first described in 1992 as a subunit of the hnRNP complex. The protein $\mathrm{K}$ element in particular is highly conserved across species and is found in multiple subcellular compartments (nucleus, cytoplasm, mitochondria). This protein has been shown to bind to a vast array of molecular targets including DNA, RNA, kinases, transcription factors, translation factors, splicing proteins, a methyltransferase, and a helicase. Like Cd002 (one of the GC-binding proteins that we identified), hnRNP K is able to bind eF-1 $\alpha$ and plays a role in translation $(\underline{93}, \underline{94})$. It has been shown to bind the promoter region of $\mathrm{c}-\mathrm{myc}$ and to regulate its transcription. hnRNP $\mathrm{K}$ is dysregulated in a number of different cancer types, and it has been reported as a prognostic indicator for colorectal cancer $(\underline{95-100})$. It also may be involved in viral infection. In one specific example, the FLIP antiapoptotic protein is believed to be a prognostic indicator for nasopharyngeal carcinoma; hnRNP K and nucleolin appear to work together to regulate the transcription of FLIP $(\underline{100})$. Isoform 2 (along with isoform 1) is the most abundant species and appears to be the predominant form of hnRNP K found in the cytoplasm, though distinct properties for this isoform are unknown (101).

Due to the diversity of molecules to which it binds, the hnRNP K protein is believed to be involved in a wide array of cellular processes. One role that is postulated is that hnRNP $\mathrm{K}$ acts as a docking platform, able to bind to a variety of players in multiple pathways and integrate their intracellular signals into a single downstream effect to the maximal benefit of the cell or organism (102).

One specific capability of hnRNP $\mathrm{K}$ is "tenacious" binding to single-stranded poly-dC nucleic acids, and not to other monobasic nucleic acid polymers - in other 
words, hnRNP $\mathrm{K}$ is one of the few known PCBPs, and the identification of hnRNP K helps to validate our pull-down assay $(\underline{103}, \underline{104})$. PCBPs are classically believed to bind to C-rich RNA species, and it is not yet proven whether or not hnNRP $\mathrm{K}$ binds particularly the i-motif structure of poly-cytosine nucleic acid, but if so it may play a role in any biological switch functions of such structures.

\section{$\underline{\mathrm{ABCF} 1}$}

The ATP-binding cassette family is a fundamental class of proteins that is conserved across species and widely expressed in human cells. Many of these proteins act as transmembrane pumps, using energy from the hydrolysis of ATP for active transport of many different molecules. There are seven distinct classes that have been identified. $\mathrm{ABCF}$ is the sixth class, also known as the GCN20 class (푸).

$\mathrm{ABCF} 1$, a member of the $\mathrm{ABCF}$ class, is also known as $\mathrm{ABC} 27$ and $\mathrm{ABC} 50$, and was first identified as a protein that is upregulated in response to tumor-necrosis factor alpha $(\mathrm{TNF}-\alpha)(105)$. It is found in the cytoplasm and the nucleus, but not the nucleolus. Unlike other members of the $\mathrm{ABC}$ superfamily, $\mathrm{ABCF} 1$ lacks a transmembrane region, and is not believed to function as a transmembrane pump. Rather, ABCF1 has been shown to play a critical role in protein synthesis and cell proliferation. It forms a complex with eIF2, GTP, and methionyl-tRNA (the initiator tRNA), and is believed to be critical to translation, initiation, elongation, and the formation of polyribosomes $(\underline{106}, \underline{107})$. It is a substrate for phosphorylation by CK2 which affects the formation of the polyribosomes with eIF2, and thus may act in the regulation of $\mathrm{ABCF} 1$ activity (106). 


\section{$\underline{\text { Nucleolin }}$}

A known generic nucleic acid binding protein. Described above as an identified GC ODN-binding protein

\section{CONCLUSIONS}

With this study, we have identified a number of proteins that bind to our GC ODN library and C monobasic ODN. Some of these proteins (nucleolin, hnRNP K) are known to bind to guanine and/or cytosine rich nucleic acid molecules. Others, although known to bind to different types of nucleic acid, have never before been implicated for these particular subtypes $(\mathrm{Ku}, \mathrm{ABCF} 1)$. One identified protein $(\mathrm{Cd} 002)$ has not previously been reported to bind to nucleic acid. Many of these protein targets are important in a wide array of biologic functions and have potentially major implications in cellular proliferation and tumor biology. The use of GC and C ODNs to target these proteins could therefore further the development of novel aptamer cancer therapeutics.

While we have identified several protein candidates, additional studies would be required in order to confirm their binding and to test for any role in biological activity. The most immediate future experiments for the completion of this project involve the verification of the protein targets identified by mass spectroscopy. We must study whether or not these protein targets are biologically pertinent by targeting living cells (both cancerous and non-malignant) rather than purified protein lysate. Cells growing in vitro would be treated with biotinylated experimental and control ODNs, then lysed and bound proteins purified via biotin-streptavidin association as before. Following this, we would perform Western blotting with target-specific antibodies against the proteins 
identified by mass spectroscopy. In this way we could assure that the proteins previously isolated from the whole cell lysate are indeed targeted by our ODNs of interest within the context of a living cell and may potentially play a role in their mechanism of action or lack thereof.

The following step would be to examine the relevance of the identified protein targets of the GC library to its profile of antiproliferative activity across different cancer types. We are particularly curious about the GC library's markedly attenuated antiproliferative activity against the MCF7 breast cancer cell line (manuscript, Appendix A). An important question to answer is whether or not the proteins of interest are present in differing levels in the growth-inhibited lung and prostate cancer cells versus the less responsive breast cancer and virtually unresponsive fibroblast cells. If so, these data could shed light on the mechanism of the antiproliferative activity of GC ODNs. The specific experiments would depend on the particular protein target and its pathways, but could include such classic molecular biology techniques such as analysis of gene expression, a functional assay if one exists, and gene silencing or upregulation of the purported target to see if the antiproliferative activity can be modulated.

For example, a possible experimental plan to study the GC library targeting of the $\mathrm{Ku}$ proteins is as follows:

- Determine baseline levels of Ku70 and Ku80 in untreated DU145, MCF7, and Hs27 cells by Western blotting and/or qPCR. Treat cells with GC 26mer, N26mer, AS1411, and no ODN, and repeat experiment to determine effect on Ku70 and Ku80 expression.

- Treat DU145 prostate cancer cells in culture with biotinylated GC 26mer, N 26mer, 
AS1411, and no ODN. Control cell lines (Hs27 and/or MCF7) may also be employed.

- Make cell extracts from treated cells and isolate proteins bound to biotinylated ODNs as before.

- Perform Western blotting for Ku70 and Ku80 to validate our previous identification of these proteins and determine variations in extracted protein levels.

- Decrease and increase expression levels of Ku70 and Ku80 in DU145 cells using siRNA and expression plasmids (with control molecules)

- Perform MTT antiproliferative and flow cytometry on these modulated cells to determine the effect of levels of Ku70 and Ku80 on GC 26mer activity and uptake. Use appropriate control ODNs and cells.

Another interesting follow-up project would be to determine what specific sequence(s) within the GC 26 mer library bind to these identified GC-binding proteins. The most logical course is to perform SELEX with the GC 26mer library upon the identified protein target and isolate unique sequences that bind it. We would screen these candidate aptamers for high affinity binding to the protein target. The evolved sequences that are in fact aptamers for our target could then be taken through the same biophysical and biological studies as for the parent library to determine the antiproliferative activity, structure, stability, cellular uptake, and protein binding of these new potential therapeutics.

A final possible study could be to test the G-rich GC 26 mer library in comparison with the G-rich GT library for whole cell SELEX. It would be interesting to compare the candidate aptamers evolved from the GC library versus both the classic $\mathrm{N}$ library and the GT library. Outcomes could include the speed (i.e. number of SELEX cycles) with which 
candidate aptamers are identified, location and prevalence of guanines within the candidates, and increases in cellular uptake and antiproliferative activity relative to the parent libraries. Given the attenuated activity of the GC library in breast cancer cells, one could argue that GC aptamers are therefore ill-suited for SELEX to find aptamers specific for that tumor type. However, the partial antiproliferative activity of the GC library against that cell line suggests that it does indeed contain sequences that inhibit the growth of those cells. Perhaps the GC library contains more potential anti-breast cancer sequences than the $\mathrm{N}$ library, but fewer than the GT library. We could perform whole cell SELEX with these three libraries on breast cancer cells to study how the antiproliferative activity of the parent library affects the SELEX outcomes.

With the data we have generated from this project involving ODN libraries (Chapters 2 and 3), there are a plethora of possible experiments we could perform that would help elucidate the nature of G-rich libraries, G-rich ODNs, proteins that bind to them, and their mechanisms of action. We decided instead to refocus our efforts on the specific aims of this project and attempt the generation of new anti-prostate cancer aptamers. 


\section{CHAPTER 4}

\section{IDENTIFICATION OF A NEW ANTI-PROSTATE CANCER \\ APTAMER BY SELEX}

\section{INTRODUCTION}

\section{Therapeutic applications of aptamers}

The field of aptamers (oligonucleotides that form specific three-dimensional structures and have target-binding specificity) is new and largely untapped. In terms of clinical use, aptamers could potentially be used in a multitude of ways: as imaging agents, as vectors for the targeted delivery of drugs, as histology stains for tissue specimens, and as bona fide pharmaceuticals. Relative to monoclonal antibodies, aptamers are inexpensive and simple to manufacture. Relative to small molecule drugs, they are target-specific and because of this have few side effects. Aptamers are more easily modified than either of these two other classes of drugs such that the development of one target-specific aptamer could easily result in more than one application simply by the affixation of different molecules; an aptamer that binds to cancer cells could both be tagged with a radionuclide for use in tumor imaging and be conjugated to a drug with poor tumor specificity resulting in targeted delivery of that drug.

\section{Aptamers as chemotherapy}

We believe there is immense potential in the development of new aptamers for 
clinical use, especially in the management and treatment of cancer. Chemotherapy regimens are notoriously difficult for cancer patients to tolerate. The side effects of these treatments can be so debilitating that doses must be limited to less than the recommended therapeutic level with correspondingly less anticancer efficacy. Consideration for nonspecific toxicities is particularly important in patients with poor reserves, such as those who are elderly or have other comorbidities (e.g. obesity, heart failure, chronic obstructive pulmonary disease, high blood pressure). In today's aging and increasingly inactive American society, an ever higher percentage of patients are clinically compromised in these ways. Physicians, patients, and patients' caretakers together must consider the impact of chemotherapy on the patients' quality of life versus any possible extension of life, and strike a careful balance between maximizing the treatment for the malignancy while keeping the side effects to a tolerable level.

Even when the recommended doses of chemotherapy are able to be administered, there remains much room for improvement in the generally poor efficacy of most chemotherapy regimens. For example, almost half of young patients with early stage breast cancer (age less than 50 years; disease restricted to the breast and locoregional lymph nodes such that surgery can be curative) will die from their malignancy within fifteen years. Adjuvant chemotherapy has been shown to decrease the likelihood of recurrence in these patients, but there has been only a modest effect on overall survival ( $42.4 \%$ vs. $32.4 \%$ mortality at 15 years). The effect is even less robust in older patients (50-69 years old; 50.4 vs. $47.4 \%$ mortality at 15 years) $(\underline{108})$. For metastatic castration resistant prostate cancer (CRPC), two landmark clinical trials reported a significant increase in survival with docetaxel in 2004 . The findings were so compelling that the 
standard of care for CRPC was quickly revised to incorporate the new data. While any improvement in overall survival is good news, the sad fact remains that even this high profile, high impact result translates to an increase of only 2-3 months of life (18.9 months vs. 16.4 months $)(4,5)$. Three new drugs for the definitive treatment of metastatic CRPC have been approved in the last year, each of which may confer an additional few months' survival (). $)$ These drugs provide important further opportunities for CRPC patients, but it is clear that although progress is being made, we must strive for even greater advancements in the treatment of patients with cancer.

The goals in the development of new cancer drugs must therefore be twofold: first and foremost, treatments should positively and significantly impact patient survival. Second, treatments must have tolerable side effects that have been minimized as much as possible. Aptamer drugs may be particularly well suited to meet these two criteria. In terms of efficacy, aptamers may be engineered against virtually any target, including any identified tumor specific antigen. If such an antigen is truly a valid target in the treatment of cancer, an aptamer that is able to be delivered to that target and specifically bind it will be a valuable pharmaceutical tool. Aptamers also typically have very few side effects, likely for multiple reasons including target specificity, lack of immunogenicity, small size, and rapid systemic clearance. In clinical trials, aptamers consistently exhibit large therapeutic windows and comfortable safety margins $(\underline{17}, \underline{109}, \underline{110})$. The Phase I clinical trials of AS1411 were remarkable not only for the tumor regression seen in multiple patients, but also for the virtual absence of major adverse events such that no maximum tolerated dose (the dose at which toxicities are no longer acceptable) was seen; in other words, all the patients were easily able to tolerate even high doses of AS1411 $(\underline{17}, \underline{38})$. 


\section{Selective evolution of ligands by exponential enrichment (SELEX) on whole prostate}

cancer cells

As discussed in Chapter 1, aptamers have an additional advantage over both antibodies and small molecules (i.e. traditional classes of chemotherapy) in that they may be engineered to bind specific targets with relative ease. The most powerful aptamergenerating technology is SELEX, an iterative and evolutionary method to screen large pools of billions of different sequences for aptamers that specifically bind a target of interest (for a more detailed description of the basic SELEX methodology, see Chapter 1, Figure 1). Many features of SELEX may be fine-tuned by the user. For example, the lengths of the variable and the primer-binding regions may be adjusted as shorter aptamers are less expensive to manufacture. It has also been postulated that minimizing the lengths of the primer regions reduces non-specific binding and experimental artifact (111). For the critical step of purification of single-strand molecules from the PCR product, different methods have been described including asymmetric PCR, biotinylated primers and streptavidin beads or fixed matrix, gel electrophoresis, and lambda exonuclease digestion, each with different advantages and disadvantages (112).

In the years since its inception in 1990, a plethora of major variations of the basic SELEX procedure have been developed (113):

$\underline{\text { Library modifications }}$

- Tailored SELEX - To reduce primer-based artifact, primer binding regions are ligated to primerless ODNs before the PCR reaction, then removed from the amplified product before the next round of selection $(\underline{114}, \underline{115})$. 
- Genomic SELEX - To identify endogenous RNA aptamers and the proteins that bind to them, the starting library is based upon the genome of the organism of interest. This method can identify protein-binding RNAs and elucidate significant nucleic acid regulatory pathways $(\underline{116}, \underline{117})$.

Separation of bound versus unbound ODNs

- Capillary electrophoresis (CE) SELEX - Sequences that bind the target of interest experience a motility shift which is exploited during the separation of bound versus unbound ODNs using capillary electrophoresis (118-120).

- Micromagnetic separation (MMS) SELEX - Disposable microchips with large magnetic field gradients are used to separate sequences bound to targets affixed to magnetic beads $(\underline{121}, \underline{122})$.

- Monolex - ODNs are passed through an affinity column and sorted into unbound, low affinity and high affinity fractions. The fraction containing the highest affinity sequences are amplified once and tested $(\underline{123})$.

Types of targets $(124)$

- Counter SELEX - Many targets are only subtly distinguishable from a related counterpart (e.g. disease prion protein vs. endogenous prion protein, ATP vs. ADP). To increase target-specificity, the ODN pool is applied to the negative target to remove sequences that are able to bind to this undesired target. Unbound ODNs are recovered and then applied to the positive target $(\underline{125}, \underline{126})$.

- Toggle SELEX - The ODN pool is applied to different protein targets in alternation, for example human vs. murine epitopes, to select for aptamers that will have crossreactivity (127). 
- Cell-SELEX - Discussed in greater detail below.

Cell-SELEX, the variation that may prove particularly relevant for the generation of clinically useful aptamers, involves the nature of the target. Rather than using a purified molecule as the substrate upon which the ODN library is applied, the target is whole cells in culture $(\underline{128-130})$. Both prokaryotic and eukaryotic cells have been targeted. The method of cell-SELEX has the advantage of applicability; instead of targeting a molecule that may or may not ultimately prove to be clinically useful, the cell itself is targeted. With proper selection, the evolved aptamers are able to bind target cellspecific antigens. It is not necessary to know the identity of the antigen; indeed, cellSELEX may very well result in the identification of a novel biomarker. Moreover, the aptamers are selected to bind the antigens in their native configuration in their natural in situ environment. Experiments may be performed in serum-containing cell-culture medium such that sequences are automatically selected for nuclease resistance as well as target specificity. Cell-SELEX has been used to identify aptamers that bind to such targets as $S$. aureus bacterium, T. brucei parasite, influenza virus, endothelial cells, leukemia cells, and glioblastoma cells $(\underline{19}, \underline{125}, \underline{130-132})$.

We have undertaken two particular innovations to the cell-SELEX technique. The first is to amplify only those sequences internalized by the cell as opposed to simply binding to the cell surface. The overall objective of this project is to develop new targeted aptamers specific for the treatment of prostate cancer, and the major goal of this portion of the project is to identify aptamers that are efficiently able to enter prostate cancer cells. One of the major hurdles of nucleic acid therapies of all types is poor cellular uptake. Although aptamers don't necessarily require uptake in order to function, any aptamer 
than binds an intracellular target requires a mechanism to cross the cell membrane. An exciting potential utility for aptamers with naturally high levels of intracellular uptake is as vehicles for other drugs with poor uptake, including almost all other oligonucleotide therapies (antigene, antisense, RNA silencing, and transcription decoy strategies, discussed in Chapter 1). For therapies that do not require a vector to become internalized, a targeted aptamer that is able to achieve high levels inside a cell may still enhance their delivery and result in higher cellular concentration. Additionally, should the aptamer be cancer-selective, then the appended drug need not be. Identification of cancer-selective aptamers with high levels of cellular uptake may thus both allow the introduction of molecules that are limited by uptake and confer specificity to molecules that lack it. Rather than identifying aptamers against prostate cancer cells and then modifying them downstream to enhance cellular uptake, we propose to let evolution determine from the start which of the millions of sequences within the library are most able to enter the cells. We describe our protocol for isolating only the internalized sequences in some detail below, including verification that we discarded both the unbound ODNs from the cell media and the ODNs bound to the cell surface.

Our second SELEX innovation is to test our GT library in parallel with the classic random $\mathrm{N}$ library. By starting with a library that is smaller (i.e. contains fewer different sequences) and is already enriched in molecules that can form G-quadruplexes with the desirable characteristics of thermal stability, antiproliferative activity, nuclease resistance, and enhanced cellular uptake, we hypothesized that the SELEX procedure would be more efficient and require fewer cycles before aptamers sequences can be isolated, and that the resultant aptamers would be less likely to require chemical modifications for clinical use. 
MATERIALS AND METHODS

\section{Oligodeoxynucleotides}

Oligonucleotides were purchased from Integrated DNA Technologies, Inc. (Coralville, IA) or from Invitrogen (Carlsbad, CA):

- $\quad$ library $\left(5^{\prime}-\right.$ GCG TCA TCA TCG GCT T $(\mathrm{N})_{26}$ ACG ACG ATA CTC ACG AGC - 3') where $N$ has equal probability of being A, C, G, or T

- $\underline{\text { GT library }}\left(5^{\prime} \text { - GCG TCA TCA TCG GCT T (K) }\right)_{26}$ ACG ACG ATA CTC ACG AGC - 3') where $\mathrm{K}$ has equal probability of being $\mathrm{G}$ or $\mathrm{T}$

- 5'primer (5'- GCG TCA TCA TCG GCT T - 3')

- 3' primer (5'-GCT CGT GAG TAT CGT CGT-3')

- $\quad \underline{\text { cap }}\left(5^{\prime}\right.$ - ACT ACG GAG GGG CTA GAG CAA CAA GT - 3')

- N $\quad$ 60cap $\left(5^{\prime}\right.$ - GCG TCA TCA TCG GCT TAC TAC GGA GGG GCT AGA GCA ACA AGT ACG ACG ATA CTC ACG AGC - 3')

- N 26mer $\left(5^{\prime}-(\mathrm{N})_{26}-3^{\prime}\right)$ where $\mathrm{N}$ has equal probability of being $\mathrm{A}, \mathrm{C}, \mathrm{G}$, or $\mathrm{T}$

- GT cap $\quad\left(5^{\prime}-\right.$ GGG TGG GGG TGT TGG GTG TTG GTT TG - 3')

- $\underline{\text { GT } 60 \mathrm{cap}} \quad\left(5^{\prime}\right.$ - GCG TCA TCA TCG GCT TGG GTG GGG GTG TTG GGT GTT GGT TTG GCT CGT GAG TAT CGT CGT - 3’)

- GT 26mer $\left(5^{\prime}-(\mathrm{K})_{26}-3^{\prime}\right)$ where $\mathrm{K}$ has equal probability of being $\mathrm{G}$ or $\mathrm{T}$

- $\underline{\text { AS1411 }}\left(5^{\prime}\right.$ - GGT GGT GGT GGT TGT GGT GGT GGT GG - 3')

Lyophilized libraries were resuspended in filtered-sterilized water, then heated at $65^{\circ} \mathrm{C}$ for 10 minutes and vortexed vigorously to dissolve. For uptake studies, three thymidine bases (TTT) were added to the 5' end of the library sequence to avoid quenching the 5 , 
fluorophore (FAM for flow cytometry and Alexa 488 for confocal microscopy), which occurs when immediately proximal to a G-quartet (프).

\section{Cells}

Four cell lines were used in this study: PC3 and DU145 prostate adenocarcinoma, A549 lung adenocarcinoma, and Hs27 non-malignant skin fibroblasts. All cell lines were acquired from ATCC. Cells were grown in complete cell culture medium of F-12K (for PC3) or DMEM (for all others) supplemented with $10 \%$ heat-inactivated (15 minutes at $65^{\circ} \mathrm{C}$ ) FBS and $1 \%$ penicillin/streptomycin. All cells were cultured at $37^{\circ} \mathrm{C}$ in $5 \% \mathrm{CO}_{2}$.

\section{SELEX, selecting for sequences internalized by whole cancer cells}

In a 6-well tissue culture plate, $4 \times 10^{5} \mathrm{PC} 3$ prostate cancer cells per well were plated in a volume of $1.2 \mathrm{~mL}$ complete medium and incubated overnight. For the first round of SELEX on the following day, the medium was removed, fresh complete cell culture medium was added, and ODN library synthesized with primer binding regions $(\mathrm{N}$ library, GT library, or no library) was added to a final concentration of $10 \mu \mathrm{M}$. Cells were incubated with the ODN library for one hour, then washed with PBS to remove any unbound ODNs. The cells were harvested by scraping in PBS, spinning down, and removing the supernatant. To strip away surface proteins and any surface-bound (i.e. not internalized) ODNs, cells were incubated with $0.5 \mathrm{~mL}$ of glycine- $\mathrm{HCl}(\mathrm{pH} 5.0)$ for 5 minutes. Cells were washed thrice with PBS, and finally incubated overnight at $55^{\circ} \mathrm{C}$ with Proteinase K (0.1 mg / mL, Invitrogen) in PK buffer (15 mM Tris-HCl, (pH 8.0), $50 \mathrm{mM}$ $\mathrm{KCl}, 2.5 \mathrm{mM} \mathrm{MgCl}, 0.5 \%$ Tween 20)). The following day, samples were spun down to 
pellet cell debris and the cell lysate supernatant transferred to fresh tubes, completing the first round of SELEX.

In preparation for the second round, the lysate was used as template for PCR to amplify the internalized sequences. PCR reaction tubes were set up using a PCR supermix containing enzyme and dNTPs (Invitrogen). In order to produce an excess of sSDNA product, the primers corresponding to the ODN libraries' primer binding regions were added at a ratio of $20: 1(4 \mu \mathrm{M}$ vs. $200 \mathrm{nM})$. The deficient primer was manufactured with a biotin tag for subsequent removal of dsDNA species from the PCR product. Five $\mu \mathrm{L}$ of lysate or water for negative control were added last. The PCR program began with 2 minutes at $94^{\circ} \mathrm{C}$ before going through 25 cycles of $25 \mathrm{sec}$ at $94^{\circ} \mathrm{C}, 25 \mathrm{sec}$ at $55^{\circ} \mathrm{C}, 30$ sec at $72^{\circ} \mathrm{C}$.

To separate dsDNA from the PCR product, samples were combined with magnetic streptavidin beads (Thermo Scientific) for one hour. The supernatant containing unbound, unbiotinylated, ssDNA PCR product was removed to fresh tubes. Approximately one tenth of this purified product $(5 \mu \mathrm{L})$ was added to new cells for the subsequent round of SELEX. The remaining cell lysate was stored at $-20^{\circ} \mathrm{C}$.

\section{Radioactive PCR to detect effectiveness of final wash before lysis}

To ensure that we enrich the sequences that have been internalized by the cells, we performed PCR to detect ODNs in the cell culture medium after the addition of ODN, in the final wash before lysing the cells, and in the cell lysate. The PCR reactions were set up as described above with the addition of 2 microcuries of radiolabeled guanine nucleotide $\left(\left[\alpha-{ }^{32} \mathrm{P}\right]-\mathrm{dGTP}\right)$. Unincorporated nucleotide was removed with TE-Midi 
SELECT-D G-25 columns (Shelton Scientific, Shelton, CT) according to manufacturer's directions, then electrophoresed on denaturing $12 \%$ polyacrylamide gels. Gels were exposed to film overnight at $-80^{\circ} \mathrm{C}$ and the films developed the following day.

\section{Sequencing and selection of candidate aptamers}

To identify internalized sequences from each round of SELEX, a portion of the SELEX cell lysates were used for sequencing. PCR reaction tubes were set up using a PCR supermix containing enzyme and dNTPs (Invitrogen), SELEX primers (not biotinylated; Integrated DNA Technologies) at a final concentration of $200 \mathrm{nM}$ each, and $5 \mu \mathrm{L}$ of lysate or water for negative control. The PCR program was as described for SELEX with the addition of a final step of 10 minutes at $72^{\circ} \mathrm{C}$. The PCR reactions were used directly for cloning using a TOPO vector cloning kit (Invitrogen) according to manufacturer's direction and transforming into chemically competent $E$. Coli. Colonies containing the plasmid with the PCR product insert were selected for ampicillin resistance and identified by loss of $\beta$-galactosidase function in X-gal screening. Plasmids from positive colonies were purified using a vacuum manifold 96-well high-throughput miniprep system (QIAGEN QIAprep Spin Miniprep Kit) according to manufacturer's direction which yielded approximately $250 \mathrm{ng} / \mu \mathrm{L}$ of plasmid per sample. Plasmids were sequenced at the University of Louisville Center for Genetics and Molecular Medicine Nucleic Acid Core facility. Approximately twenty sequences were identified from each round of SELEX. Sequences were organized by SELEX round, and analyzed for increasing prevalence of sequences and/or motifs of nucleotide bases across multiple rounds. 


\section{Cellular uptake of candidate aptamers}

Flow cytometry

Uptake experiments were performed with the two candidate aptamers ( $\mathrm{N}$ cap and GT cap) versus the respective parent libraries (N 26mer and GT 26mer). All ODNs were labeled at the 5' end with FAM (6-carboxyfluorescein). Four cell lines were studied (PC3, DU145, A549, and Hs27). For each sample, $4 \times 10^{5}$ cells were plated and incubated overnight. The following day, FAM-labeled ODNs were added to make a final concentration of 10 $\mu \mathrm{M}$ and plates were incubated for one hour. The medium containing unbound ODN was removed. Cells were washed thrice and treated with trypsin or TrypLE (Invitrogen) to remove surface-bound oligonucleotides and to harvest cells. Cells were kept on ice and protected from light until analysis. Propidium iodide ( $2 \mu \mathrm{g}$ per sample) was added as a marker of viability. Ten thousand cells were counted after gating to exclude PI-positive (non-viable) cells from analysis. Cells were analyzed using a FACScan cytometer. The mean fluorescence of cells for each sample was determined using the FlowJo software program.

\section{Confocal microscopy}

Three ODNs samples were manufactured with conjugated Alexa 488: N 26mer, GT 26mer, and GT cap. Thirty thousand PC3 cells were plated in each chamber of fourchambered microscopy slides and incubated overnight. The following day, Alexa 488 ODNs were added to a final concentration of $10 \mu \mathrm{M}$ and incubated at $37^{\circ} \mathrm{C}$ for 2 hours. Cells were washed thrice, fixed with $4 \%$ paraformaldehyde for 25 minutes, and washed thrice more. Coverslips were applied to chambers with a drop of Antifade containing 
DAPI (Millipore). Cells were analyzed by confocal microscopy using a LSM 510 inverted confocal laser scanning microscope (Carl Zeiss).

Quantitative PCR of lysates of cells exposed to ODNs

PCR requires the use of primer binding regions. Therefore, candidate aptamers were ordered as 60mers (N 60cap, GT 60cap) comprised of the captamer sequence (N cap or GT cap) with flanking primer binding regions as for the parent 60mer ODN libraries.

To prepare cell lysates for qPCR analysis for ODN uptake: In a 6-well tissue culture plate, $4 \times 10^{5} \mathrm{PC} 3$ prostate cancer cells per well were plated in a volume of 1.2 $\mathrm{mL}$ complete medium and incubated overnight. On the following day, the medium was changed and ODNs added (N 60mer, N 60cap, GT 60mer, or GT 60cap) to a final concentration of $10 \mu \mathrm{M}$. Cells were incubated with the ODN library for one hour, then washed with PBS to remove unbound ODNs. The cells were harvested by scraping in PBS, spinning down, and removing the supernatant. To strip away surface proteins and any surface-bound (i.e. not internalized) ODNs, cells were incubated with $0.5 \mathrm{~mL}$ of glycine- $\mathrm{HCl}(\mathrm{pH} \mathrm{5.0)}$ for 5 minutes. Cells were washed thrice with PBS, and finally incubated overnight at $55^{\circ} \mathrm{C}$ with Proteinase $\mathrm{K}(0.1 \mathrm{mg} / \mathrm{mL}$, Invitrogen) in PK buffer $(15$ $\mathrm{mM}$ Tris- $\mathrm{HCl}$, ( $\mathrm{pH} 8.0$ ), $50 \mathrm{mM} \mathrm{KCl}, 2.5 \mathrm{mM} \mathrm{MgCl}$, 0.5\% Tween 20)). The following day, samples were spun down to pellet cell debris and the cell lysate supernatant transferred to fresh tubes.

For the qPCR reactions, lysates were prepared at six ten-fold dilutions (neat $1: 10^{5}$ ) and plated in triplicate with appropriate negative controls in a 96-well qPCR plate. Reactions were set up using a 2x SYBR PCR master mix (Applied Biosystems) 
according to manufacturer's directions. Samples were run on an Applied Biosystems 7500 Real-Time PCR System machine.

\section{Mechanistic studies of candidate aptamers (GT cap and $\mathbf{N}$ cap)}

MTT anti-proliferative assay

The candidate aptamers and their parent libraries were tested in triplicate at eight concentrations $(0,2,4,6,8,10,12,14 \mu \mathrm{M})$ in four cell lines: PC3, DU145, A549, and non-cancerous Hs27. We modified previously published MTT [3- (4,5-Dimethylthiazol2-yl)-2,5-diphenyltetrazolium bromide] assay protocol (16). Briefly, cells were seeded in 96-well tissue culture plates and incubated overnight. To account for intrinsic differences in growth rate, the A549 and DU145 lines were plated at 1000 cells per well, PC3 at 2000 per well, and Hs27 at 3000 per well. ODN stock solutions were then added to each well to yield the desired final concentration. Plates were incubated with the ODN for five days, during which the cell culture medium was not changed. On day five, cell viability was determined and the background corresponding to medium alone (no cells) was subtracted. For each concentration, the average of the three wells was calculated and plotted. Assays were repeated to ensure reproducibility.

\section{Circular dichroism spectroscopy}

Candidate aptamers and their parent libraries were diluted to a concentration of $5 \mu \mathrm{M}$ in $1.5 \mathrm{~mL}$ of either water, or $100 \mathrm{mM}$ sodium chloride, or $100 \mathrm{mM}$ potassium chloride. Samples were incubated at $95^{\circ} \mathrm{C}$ for 5 minutes, then cooled on ice until analysis. CD spectra were collected at $20^{\circ} \mathrm{C}$ using a Jasco J-715 spectropolarimeter between 340 and 
$220 \mathrm{~nm}$ wavelengths, using $200 \mathrm{~nm} / \mathrm{min}$ scanning speed, 2 s response time, $5 \mathrm{~nm}$ bandwidth, and $1 \mathrm{~cm}$ pathlength. For each set of samples, the same solution used to prepare the ODNs was used as the reference.

Protein binding to whole cell lysate using electromotility shift assay (EMSA)

Candidate aptamers were tested in comparison with their parent libraries. Each ODN was 5 '-end-labeled with ${ }^{32} \mathrm{P}$ using $\mathrm{T} 4$ kinase (Invitrogen). Unbound isotope was removed from samples using TE-Midi SELECT-D G-25 columns (Shelton Scientific, Shelton, CT) according to manufacturer's directions. Collection tubes containing end-labeled libraries were kept on ice, or stored at $-20^{\circ} \mathrm{C}$ for up to one day. The radioactivity of end-labeled libraries was measured in a scintillation counter on the day of the experiment. Whole cell lysates from PC3 cells were prepared as follows: Cells were grown till confluent, then washed and scraped with HEDG buffer (20 mM HEPES-NaOH, pH 7.8, 0.5 mM EDTA, $0.5 \mathrm{mM}$ dithiothreitol (DTT)) containing $10 \%$ glycerol, $1 \mathrm{x}$ protease inhibitor cocktail (Sigma, St. Louis) and $0.42 \mathrm{M} \mathrm{NaCl}$ at a ratio of $100 \mu \mathrm{L}$ per $75 \mathrm{~cm}^{2}$ culture area. The solution was harvested and subjected to three freeze-thaw cycles (liquid nitrogen versus room temperature). Samples were centrifuged at $16,000 \times \mathrm{g}$ at $4^{\circ} \mathrm{C}$ for 45 minutes to pellet cell debris. An equal volume of HEDG with glycerol and protease inhibitor but without $\mathrm{NaCl}$ was added to reach a final concentration of $0.21 \mathrm{M} \mathrm{NaCl}$. Fifty thousand cpm of radiolabeled-ODN were combined with $2.5 \mu \mathrm{g}$ of cell extract and $5 \mathrm{x}$ protein binding buffer ( $1 \mathrm{x}$ is $20 \mathrm{mM}$ Tris- $\mathrm{HCl}$, pH 7.4, $140 \mathrm{mM} \mathrm{Kl}, 2.5 \mathrm{mM} \mathrm{MgCl}, 1 \mathrm{mM}$ dithiothreitol, 0.2 mM PMSF [phenylmethylsulfonyl fluoride]). Samples were incubated at $37^{\circ} \mathrm{C}$ for 15 minutes, then run on a $5 \%$ non-denaturing polyacrylamide gel. The gel 
was fixed in a solution of $10 \%(\mathrm{v} / \mathrm{v})$ methanol $+10 \%(\mathrm{v} / \mathrm{v})$ glacial acetic acid in water for $10 \mathrm{~min}$ at room temperature, then exposed to a storage phosphor screen for 2 hours at room temperature before scanning.

Nuclease-resistance in serum-containing medium and PC3 cytoplasmic extract Four ODN samples were studied: N 26mer, N cap, GT 26mer, and GT cap. The ODNs were 5 '-end-labeled with ${ }^{32} \mathrm{P}$ using T4 kinase (Promega) and unincorporated $\gamma-{ }^{32} \mathrm{P}$-ATP was removed from samples using TE-Midi SELECT-D G-25 columns (Shelton Scientific, Shelton, CT, USA) according to manufacturers' directions. Collection tubes containing end-labeled samples were stored at $-20^{\circ} \mathrm{C}$. The radioactivity of end-labeled samples was measured in a scintillation counter on the day of the experiment. For each ODN or library to be tested, four reaction tubes containing complete cell culture medium without antibiotics and four reaction tubes containing $2.5 \mu \mathrm{g}$ PC3 cytoplasmic extract in DMEM were prepared. Fifty thousand counts per minute of end-labeled ODN were added to each of four tubes. Samples were incubated at $37^{\circ} \mathrm{C}$, and tubes were flash-frozen in a dry ice/ethanol bath at $0,1,4$ and $24 \mathrm{~h}$. Frozen samples were stored at $-80^{\circ} \mathrm{C}$ until all samples had been harvested. Samples were then thawed on ice and run on $12 \%$ denaturing polyacrylamide gels. Band density was measured using the UN-SCAN-IT gel densitometry application (Silk Scientific, Orem, UT, USA). The percent sample remaining was calculated taking the signal of the zero time point lane to be $100 \%$.

\section{RESULTS AND DISCUSSION}

\section{Radioactive PCR indicates success of washes and isolation of internalized ODNs}


Our goal was to isolate aptamers that were efficiently able to enter the cell. Our cell-SELEX protocol must therefore include not only washing unbound ODNs away from the prostate cancer cells, but also stripping away any surface bound molecules. Different methods have been employed for this purpose. At the beginning of this project, we tested both the glycine method described above (harvesting cells by scraping and treating with glycine $\mathrm{HCl}$ ) and a standard trypsin digestion to both digest surface epitopes (along with any bound ODNs) and resuspend the cells. An initial round of cell-SELEX was performed and the final wash and the cell lysate from both methods tested by radioactive PCR for the presence of ODNs. Samples were run on denaturing polyacrylamide gels (Figure 1). A detectable band was amplified from the cell lysate but not the final wash for the samples from the glycine-treated cells, indicating that ODNs were present in the cell lysate after removing cell debris, and that the washing process was effective at removing all detectable ODNs after incubation with glycine. The converse was true for samples from the trypsin digest, indicating the presence of ODNs in the final wash and a dearth of ODNs in the cell lysate. A possible reason for these data is that that the trypsin permeabilizes the cells such that internalized ODNs are able to leak out, thus increasing the amount of amplifiable ODNs in the wash and decreasing the amount in the lysate. We concluded that the trypsin digest was not optimal for our purposes, and utilized the glycine stripping method for the isolation of internalized aptamers using the cell-SELEX procedure.

\section{Candidate aptamers identified from both the N library and the GT library}

On the order of 20 samples from the cell lysates of each round of SELEX were 


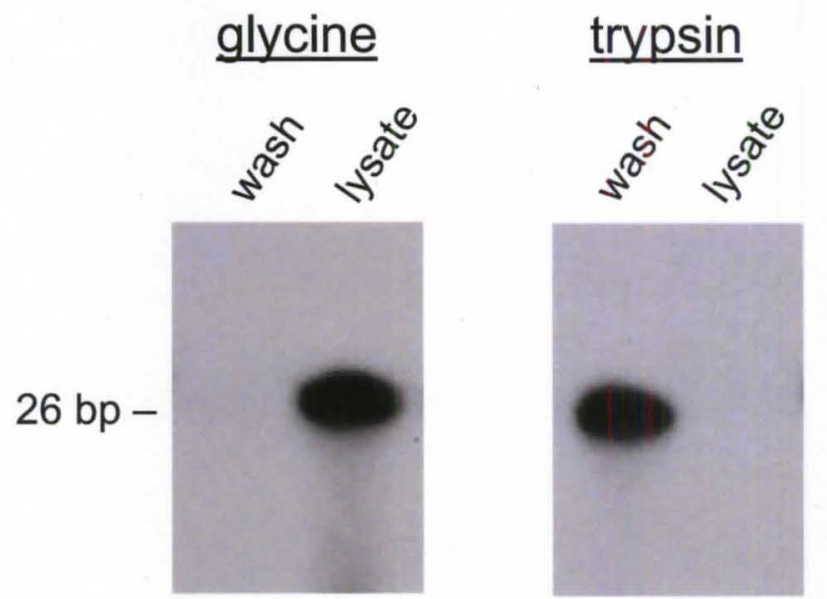

Figure 1: Radioactive PCR of final wash and lysate. In optimizing our SELEX protocol to select for internalized ODNs, we tested two different methods to strip away surface-bound molecules (glycine vs. trypsin). The final wash after stripping was clean of ODNs for the glycine strip, but not for the trypsin. The crude cell lysate contained detectable ODNs for the glycinetreated cells, but not the trypsin-treated cells.

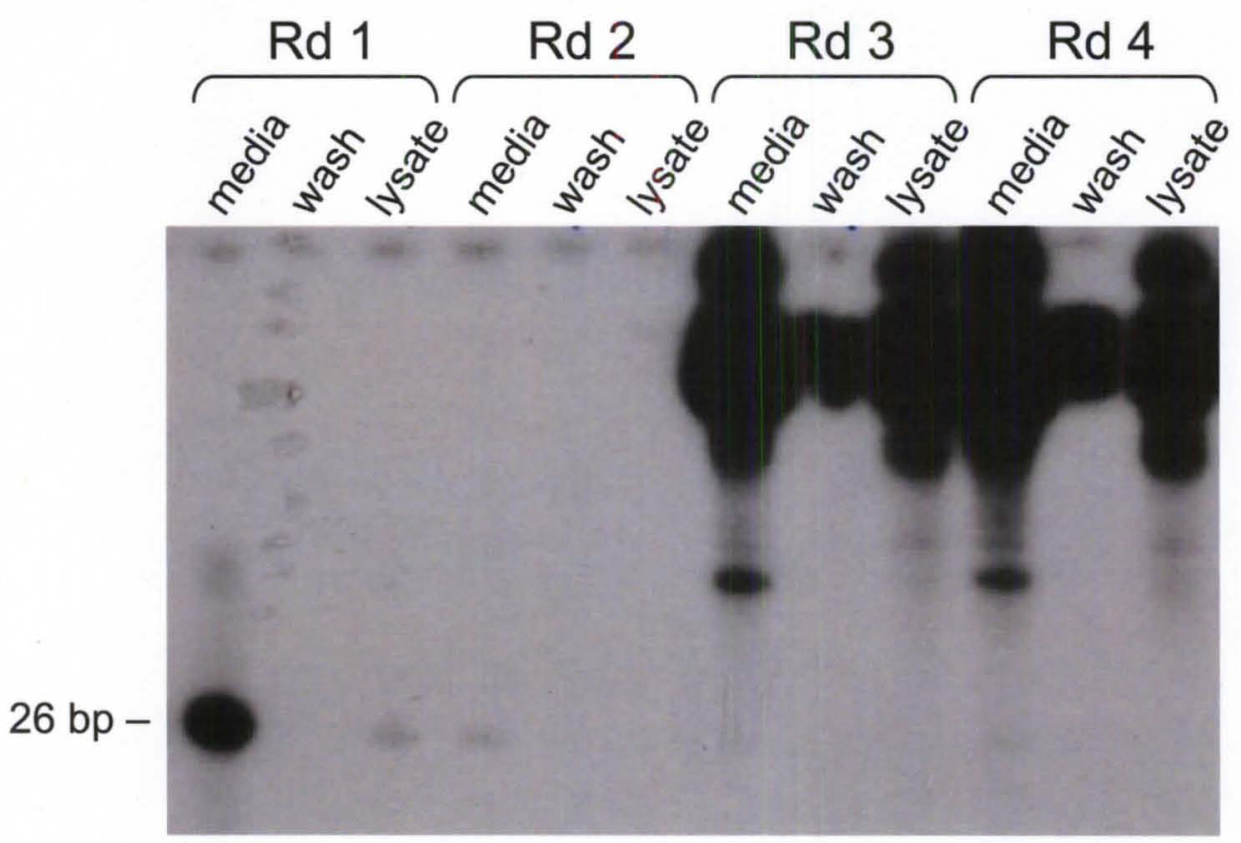

Figure 2: Radioactive PCR from sequential rounds of SELEX. In round one, a 26mer band (the length of the ODN starting library) was detectable in the cell culture media after adding the ODN library, and in the cell lysate after 1 hour incubation. In later cycles, bands of much higher molecular weight were strongly present while the $26 \mathrm{mer}$ band was undetectable. These data suggest the production of experimental artifact with subsequent cycles. 
sequenced, yielding a long but by no means exhaustive list of ODNs (Appendix B). As expected, in the first two rounds of SELEX for the $\mathrm{N}$ library there were few sequences that appeared more than once, implying that many more ODN species were present that were not selected for sequencing, and that no particular sequence was yet enriched. We were surprised to see some sequences repeated from multiple bacterial clones as early as round 3 , indicating that those sequences were becoming more prevalent in the ODN pool. By rounds 4 and 5, the $\mathrm{N}$ library candidate aptamer (N cap: 5' - ACT ACG GAG GGG CTA GAG CAA CAA GT - 3') was virtually the only 26 mer that was present.

It was more difficult to identify candidates from the GT library; as every sequence is made of combinations of $\mathrm{G}$ and $\mathrm{T}$ bases, patterns of bases are more inscrutable. We identified some common base motifs among the sequences that appeared to become enriched over subsequent cycles. From these, we composed a candidate aptamer (GT cap: 5' - GGG TGG GGG TGT TGG GTG TTG GTT TG - 3'). These two candidate aptamers, N cap and GT cap, were characterized using the same panel of assays we utilized for our ODN libraries study and tested for our SELEX selection criterion of enhanced cellular uptake in comparison with the parent libraries.

As $\mathrm{N}$ cap was the only candidate seen with the $\mathrm{N}$ library SELEX by round 5, we discontinued further SELEX cycles for both libraries. For both the $\mathrm{N}$ and the GT SELEX, we noted some obvious artifacts. An example includes an approximately 400 base sequence that evolved clearly in a textbook SELEX manner, becoming increasingly prominent with subsequent rounds (Appendix B, Round 3, Sequence 2). There were no obvious difference in either frequency or size of artifacts between the $\mathrm{N}$ and GT SELEX. Radioactive PCR was performed with media, final wash, and lysates from sequential 
rounds of $\mathrm{N}$ library SELEX (Figure 2). Bands at the 26mer location were seen in the media and lysates for the first two rounds, but from round 3 onward we observed several bands of much higher molecular weight in all samples. The smaller $26 \mathrm{mer}$ band was not seen, and we postulate that the larger molecular weight species are preferentially amplified resulting in loss or obscuration of the 26 mer species. This could explain the relative lack of 26 mers in the cell lysates as identified by sequencing. The denaturing gel data are largely consistent with the sequencing data.

\section{Candidate aptamers do not exhibit enhanced cellular uptake as compared to parent libraries}

Consistent with the SELEX literature, we initially focused our studies on our candidate sequences without the primer binding regions. We acquired the candidates and their parent libraries without the primer binding regions and with FAM-fluorophore tagged at the 5' end: N cap, GT cap, N 26mer, and GT 26mer. To avoid quenching, we added three thymidine bases at the $5^{\prime}$ end between the sequence of interest and the fluorophore for all four ODN samples. This modification has previously been shown to have no effect on antiproliferative activity for AS1411 and is standard operating procedure in our laboratory. PC3 prostate cancer cells were treated with these ODNs and then subjected to flow cytometry. To our chagrin, we did not see any enhancement in fluorescence with the candidate aptamers relative to their parent libraries (Figure 3A). We repeated the experiment using other cell lines (DU145 prostate cancer, A549 lung adenocarinoma, and $\mathrm{Hs} 27$ non-malignant fibroblasts). We also ordered fluorescent libraries and candidate aptamers synthesized with the primer binding regions, reasoning 
A.

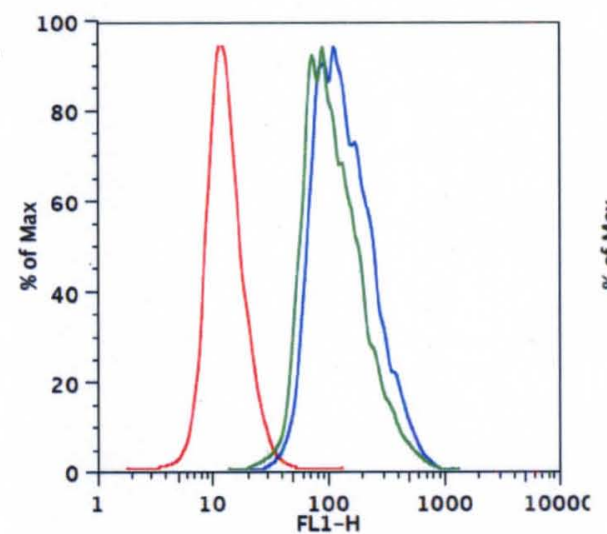

Unstained

GT cap

GT 26mer

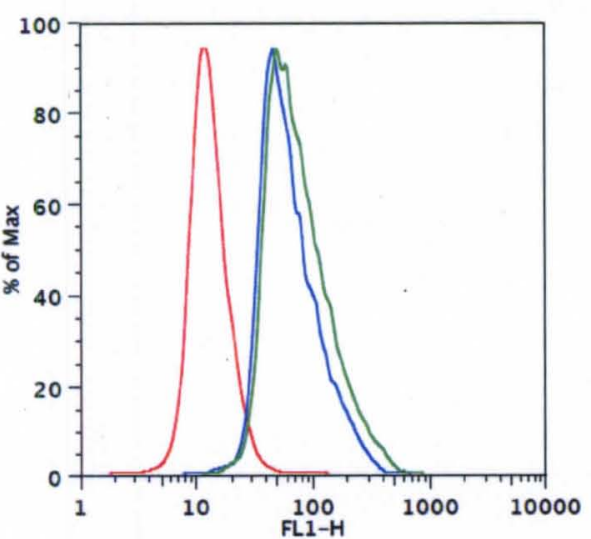

Unstained

$\mathrm{N}$ cap

N 26mer
B.

N library

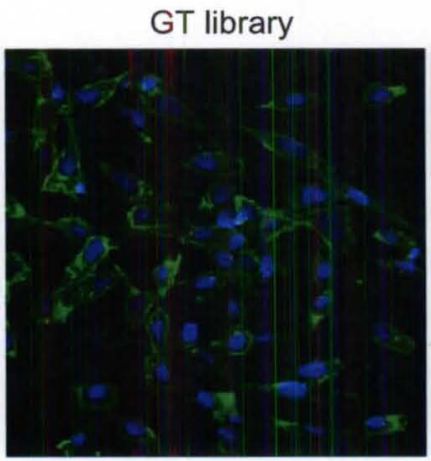

GT cap

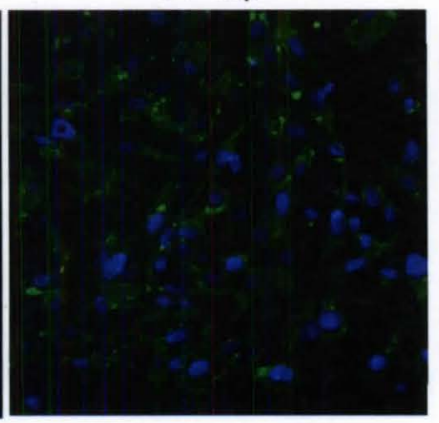

Figure 3: Cellular uptake of FAM-labeled oligodeoxynucleotides. A) Mean fluoresence in PC3 prostate cancer cells. No enhanced uptake seen by candidate aptamers. B) Confocal microscopy of PC3 cells. GT cap has less fluorescence than parent library. C) Fluorescence of ODN stocks alone show a high degree of variation. D) Cellular uptake as measured by corrected mean fluorescence in different cell lines. Correction factor based on relative fluorescence of ODN stock samples. Candidate aptamers have higher uptake than parent libraries in all cell lines. GT samples have higher fluorescence than $\mathrm{N}$ samples. Pilot experiment performed once with no statistical analysis. 
C.

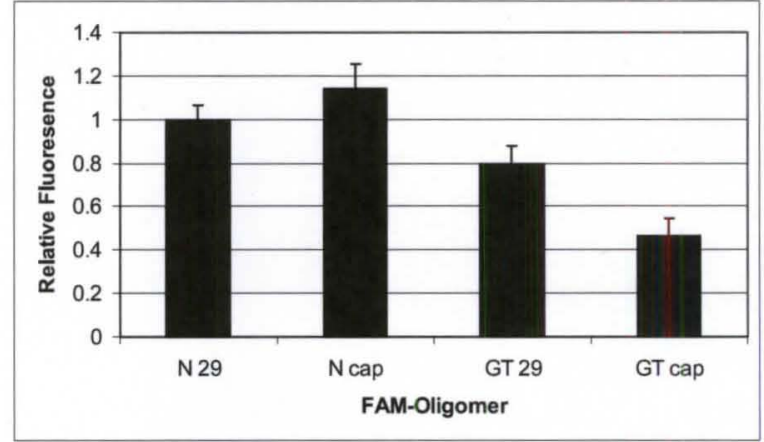

D.
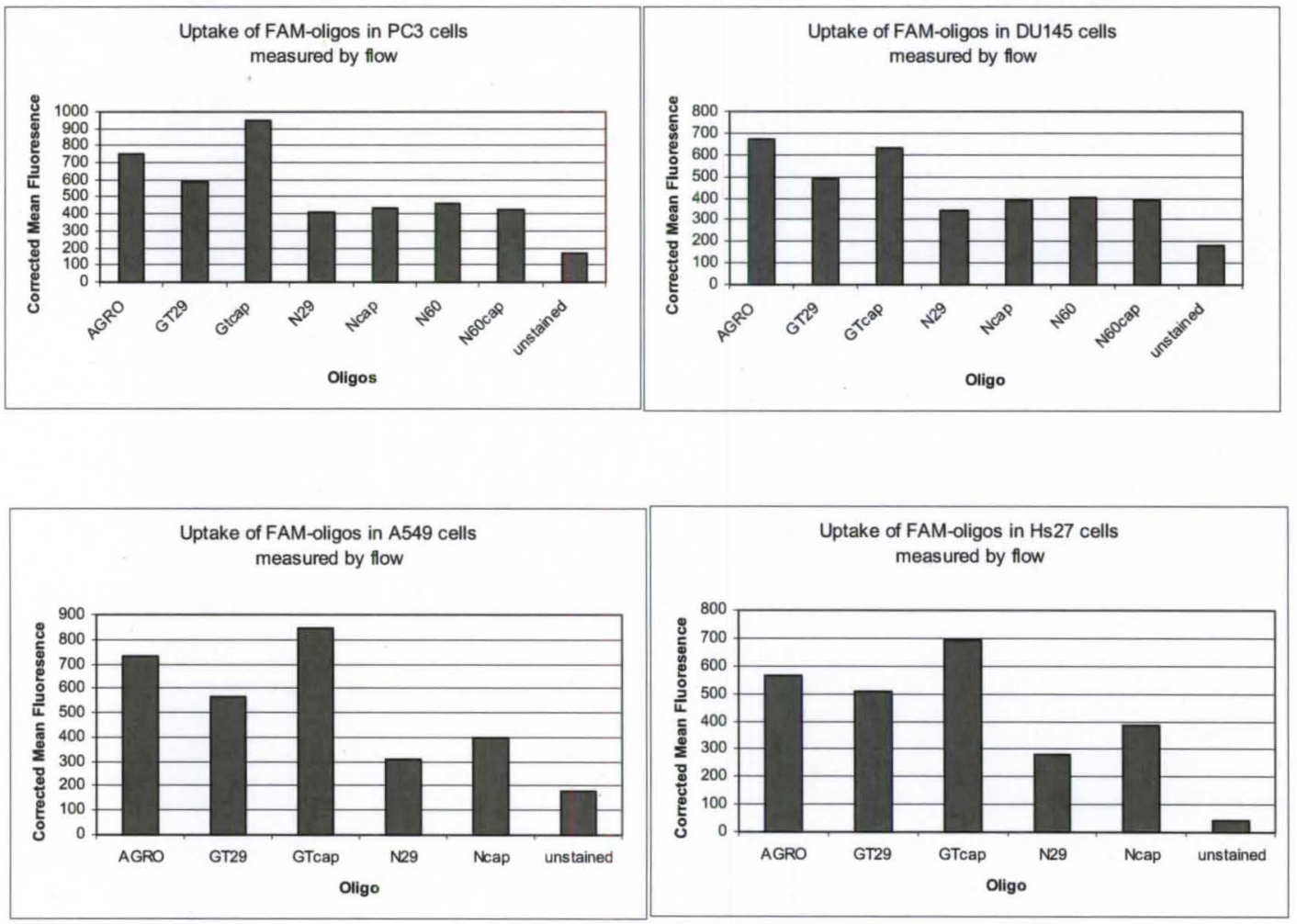

Figure 3, continued from previous page 
that the candidates' selection was performed with these additional bases and that perhaps the primer binding regions are required for cellular uptake. Even with these additional attempts, we were still unable to demonstrate enhanced cellular uptake (data not shown). We also performed confocal microscopy with the fluorophore-tagged 29 mer libraries and GT cap ( $\mathrm{N}$ cap was omitted due to consideration of cost and our particular interest in Grich ODNs), and again did not observe any enhanced activity (Figure 3B). Relative to its parent library, the GT cap sequence actually appeared to have decreased internalization as visualized by fluorescence.

We then examined the ODN stocks themselves. We observed a confounding difference in the relative fluorescence of the commercial ODN stock solutions even before applying to cells in culture (Figure 3C). In other words, some ODN stocks were inherently more fluorescent than others. This difference in relative fluorescence was most pronounced in the GT cap sample and could be due to multiple reasons including varying degrees of quenching of the fluorophore by higher order structures of the ODNs or simply to differences in coupling efficiency during synthesis. To correct for this, in parallel with the flow cytometry experiment, FAM-labeled ODNs were added in triplicate to a 96-well plate containing cell culture medium to make a final concentration of $10 \mu \mathrm{M}$ and incubated for one hour. The relative fluorescence of the samples was measured, and applied as correction factors to the measured mean fluorescence observed by flow cytometry (Figure 3D). When this method of data analysis was utilized, there was in fact enhancement of cellular uptake seen with GT cap relative to the GT 26 mer parent library. This enhancement was not exclusive to the PC3 cells, but was most marked in this line. N cap also had a modest degree of enhancement relative to the $\mathrm{N} 26$ mer library in all four 
cell lines. These data were consistent with MTT data from experiments performed in parallel (described below), and indicated that our SELEX protocol did indeed evolve aptamers with cellular uptake and that this uptake correlated well with the degree of enhancement of antiproliferative activity seen with the candidate aptamers relative to their parent libraries.

We therefore attempted to standardize both fluorescence and ODN concentration by measuring fluorescence before treating cells, ensuring that cells were treated with the same amount of measured fluorescence $\left(E_{530}=100,000\right)$, then standardizing the ODN concentration by adding unlabeled ODN as needed to a final concentration of $10 \mu \mathrm{M}$. Unfortunately, with this method we were again unable to show enhanced cellular uptake by our candidates (data not shown).

An alternate method to show cellular uptake of aptamers that does not require fluorescent ODNs is quantitative PCR. Cells were treated with candidate aptamers and their parent libraries, all necessarily with the primer binding regions affixed (i.e. 60mers). Cell lysates were prepared, and the lysates used as template in a range of dilutions for quantitative PCR using the SYBR green technology. With this protocol, sequences internalized by the cell should amplify, and a higher number of ODNs in the lysates should require fewer PCR cycles before the SYBR green signal exceeds the background threshold. Despite multiple attempts, we were unable to get reproducible data (data not shown). One replicate showed increased levels of ODN in the lysates of cells treated with GT cap relative to those treated with the GT library, but other replicates showed no difference between the samples. Other molecules within the cell lysate may have disrupted the PCR reaction. We reasoned that with increasing dilutions (1 part lysate in 
$10^{5}$ parts water), any disrupting molecules would become so sparse as to be essentially absent, but in retrospect perhaps the lack of reproducibility was due to this factor. For further attempts one should consider the purification of ODNs from the crude cell lysate. However, we did not undertake this task because of the unavoidable issue of the primer binding regions. The qPCR required the use of the primer binding regions in order to amplify the ODNs in the template sample. This may ablate any enhanced uptake of the candidate aptamers. We have performed antiproliferative MTT assays using our candidate aptamers with primer binding regions and found the GT cap had cancer-selective antiproliferative activity only without these regions (data not shown). We therefore concluded that the aptamer sequence is in fact the $26 \mathrm{mer}$, not the $60 \mathrm{mer}$, and did not further optimize this qPCR assay which would require the primer binding regions.

In summary, despite employing a number of methods, we were unable to consistently demonstrate enhanced cellular uptake by our candidates.

GT cap selectively inhibits the growth of cancer cells with greater potency than the GT library

In parallel with the cellular uptake experiments, we also undertook characterization of the candidates with the assays described in our ODN libraries manuscript (Appendix A). To assess antiproliferative activity we employed the MTT assay. We did not necessarily expect any enhancement in antiproliferative activity in our candidates relative to the parent libraries, but believed that the GT cap would likely have cancer-selective antiproliferative activity as its parent library does, while the $\mathrm{N}$ cap, as a non-G-rich sequence from a non-G-rich library, would not. A difference in 
antiproliferative activity with GT cap versus $N$ cap would illustrate the possibility of different utilities for two ODN libraries in SELEX: 1) starting with a GT library and selecting for cellular uptake to identify aptamers that may be used as cancer drugs by entering cells and halting their growth, or 2) starting with the $\mathrm{N}$ library and selecting for cellular uptake to identify aptamers that may be used as vehicles but have no intrinsic anticancer activity of their own.

We tested our ODNs on four different cell lines: two prostate cancer, one lung cancer to study organ specificity, and one non-malignant fibroblast to study cancer selectivity (Figure 4, A-D). Consistent with the SELEX literature, we focused our studies on our candidate sequences without the primer binding regions ( $\mathrm{N}$ cap and GT cap). The two parent libraries without primer binding regions (N 26mer and GT 26mer) and AS1411 were included for comparison. As before (Chapter 2), to illustrate the relative activities of the samples, we tabulate the percentage reduction in cell number at $6 \mu \mathrm{M}$ oligonucleotide concentration (Figure 4E). For stratification, we consider a reduction of $30-50 \%$ as intermediate antiproliferative activity, and greater than $50 \%$ as high.

We observed an enhancement of antiproliferative activity with the $\mathrm{N}$ cap sequence relative to the N library in the PC3 cells ( $28 \%$ versus $9 \%$ growth inhibition respectively). While the change in activity is substantial, the $\mathrm{N}$ cap sequence still had only a modest absolute effect. Additionally, the antiproliferative effect of the $\mathrm{N}$ cap ODN in the nonmalignant fibroblast line was comparable to the effects seen in the prostate cancer cells. Both the $\mathrm{N}$ cap and the $\mathrm{N}$ library had negligible effects on the growth of the lung cancer cells which may imply a degree of specificity for prostate cancer cells. We conclude that the $\mathrm{N}$ cap has only minimally enhanced antiproliferative activity over the parent library. 
A. PC3
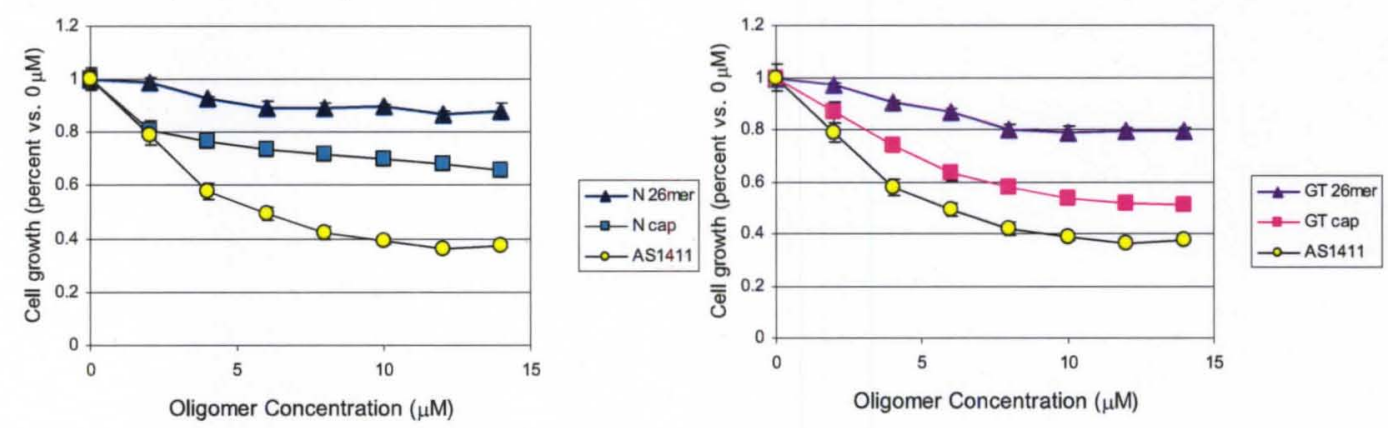

B. DU145
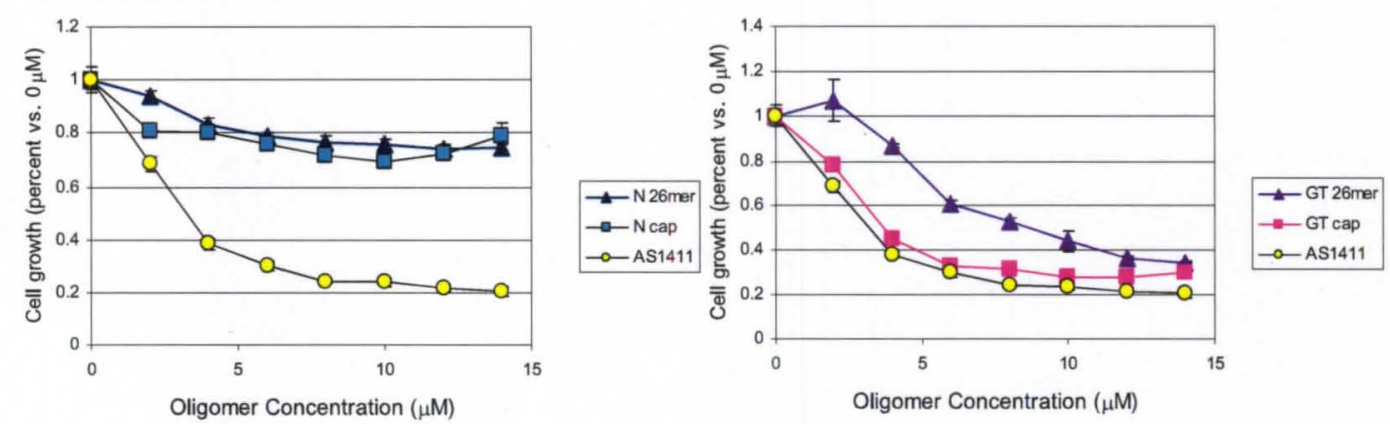

C. $\mathrm{A} 549$
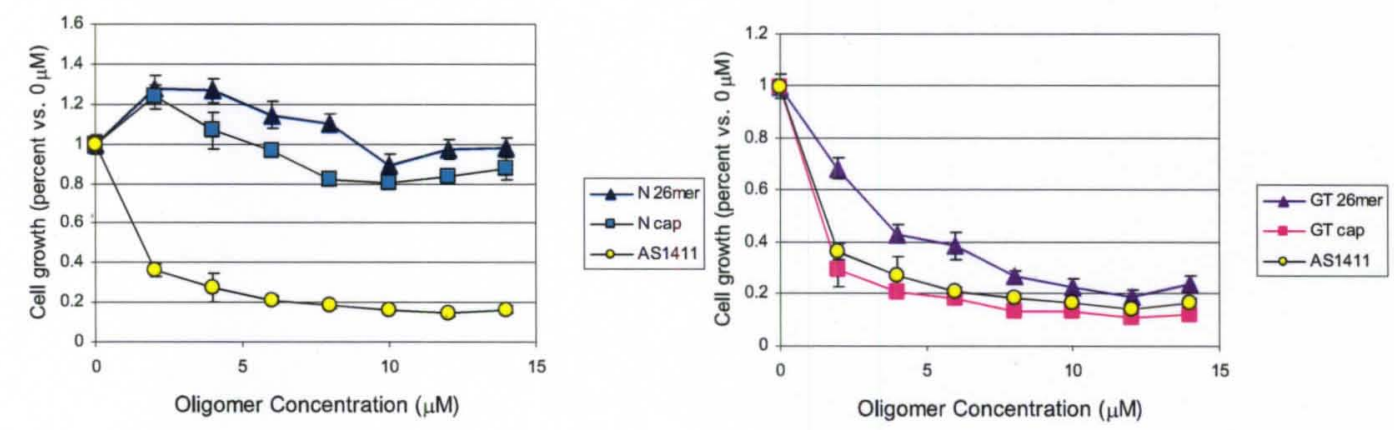

Figure 4: MTT assay of candidate aptamers compared to parent library and AS1411 on different cell types. A) PC3 prostate cancer cells. B) DU145 prostate cancer cells. C) A549 lung cancer cells. D) Hs27 nonmalignant fibroblasts. E) Tabulation of percent inhibition of cell growth at $6 \mu \mathrm{M}$ ODN. Light grey: $30-50 \%$ growth inhibition. Dark grey: $>50 \%$ growth inhibition. Experiments performed in duplicate. 
D. Hs27
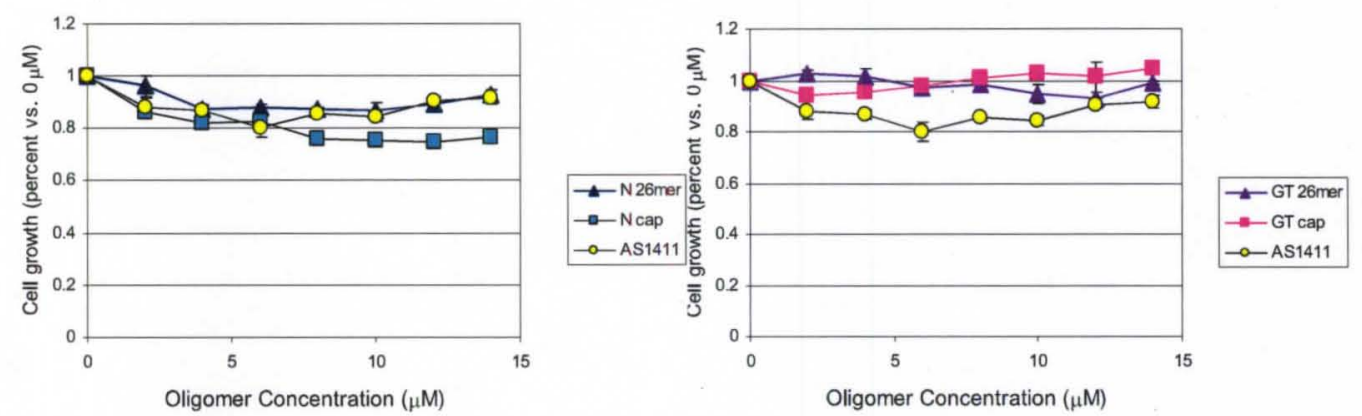

E. Percent Inhibition in Cell Number at $6 \mu \mathrm{M}$ Concentration

\begin{tabular}{|l|c|c|c|c|}
\hline Oligomer & $\begin{array}{c}\text { PC3 } \\
\text { prostate }\end{array}$ & $\begin{array}{c}\text { DU145 } \\
\text { prostate }\end{array}$ & $\begin{array}{c}\text { A549 } \\
\text { lung }\end{array}$ & $\begin{array}{c}\text { Hs27 } \\
\text { fibroblast }\end{array}$ \\
\hline N 26mer & 9 & 20 & 0 & 12 \\
\hline N cap & 28 & 27 & 0 & 21 \\
\hline GT 26mer & 13 & 30 & 65 & 0 \\
\hline GT cap & 36 & 65 & 89 & 3 \\
\hline AS1411 & 51 & 70 & 84 & 17 \\
\hline
\end{tabular}

Figure 4, continued 
In contrast, the GT cap sequence exhibited a substantial inhibition of cell proliferation that was more potent than the parent library for both prostate cancer cell lines. The fold-change was most pronounced in the PC3 cells that were used as the target of the SELEX protocol (3-fold for PC3 versus 2-fold for DU145). Despite selecting our GT candidate using prostate cancer cells, the antiproliferative effect was not limited to that organ; a similar observation was made upon testing our ODNs on lung cancer cells. Though the GT cap had the highest absolute inhibition of proliferation in the A549 lung cancer cells, the parent GT library itself has high antiproliferative activity in these cells making the fold-change seen with the selected GT cap ODN less than one. Both the GT cap and library had virtually no effect on the non-malignant fibroblasts. Excitingly, until this study we have not seen an ODN with antiproliferative activity to equal AS1411. We observe that the GT cap sequence is on par with the activity of AS1411 in both DU145 and A549 cells.

The data for the parent libraries (N and GT 26mers) in the DU145 and A549 cells were largely consistent with our previous findings (Chapter 2). As before, all libraries and ODNs have little effect on the growth of the non-malignant cells and all dose-dependence curves plateau at a non-zero value implying that the ODNs are inducing a cytostatic rather than a cytotoxic effect. We did also assay our candidates and libraries as 60 mers that included the flanking primer binding regions and tested them for antiproliferative activity in the four cell lines. No growth inhibition was seen with these ODNs (data not shown), and we conclude that it is indeed the $26 \mathrm{mer}$ sequences, not the 60 mers, that should be considered as the candidate aptamers. 


\section{CD spectra of GT cap suggests formation of the guanine-quaduplex conformation}

We have previously discussed the limitations of $C D$ data, but $C D$ is well suited to offer initial insight into the secondary structures of nucleic acids given consideration for ease and cost. In terms of G-quadruplexes, it is generally accepted that positive peaks close to either $260 \mathrm{~nm}$ or $290 \mathrm{~nm}$ are indicative of this conformation $(\underline{133}, \underline{134})$. The relative height of these peaks is influenced by the proportion of "parallel" to "antiparallel" G-quadruplexes ( $\underline{30})$. To assess the molecular structure of our ODNs and their parent libraries we studied the CD spectra in three different solutions: $100 \mathrm{mM} \mathrm{KCl}$, $100 \mathrm{mM} \mathrm{NaCl}$, and water (Figure 5).

As before, the $\mathrm{N} 26 \mathrm{mer}$ spectra are classic for unstructured nucleic acid with a negative peak at $250 \mathrm{~nm}$, a positive peak at $275 \mathrm{~nm}$, and only small changes with differences in solution. The $\mathrm{N}$ cap ODN, however, does exhibit polymorphism with solution. The peaks of the $\mathrm{N}$ cap spectra are of highest magnitude in potassium buffer, and the spectrum in this solution appears very similar to the unstructured $\mathrm{N} 26 \mathrm{mer}$ library. With water, however, the spectrum is suggestive of G-quadruplex formation with peaks at $240 \mathrm{~nm}$ and $260 \mathrm{~nm}$ wavelengths, and a positive peak at $290 \mathrm{~nm}(\underline{135})$. The spectrum of $\mathrm{N}$ cap in sodium buffer appears to be intermediate between the spectra of the

other two solutions. While the sequence of the $\mathrm{N}$ cap is not G-rich containing less than $50 \%$ guanines ( 9 guanines out of 26 bases) and is not particularly suggestive for Gquadruplex formation, it does contain a guanine-rich stretch that could contribute to this structure, especially should multiple molecules assemble. As the positive peak at $290 \mathrm{~nm}$ is less pronounced in potassium than in water, one could argue against the formation of the G-quadruplex by this sequence. Alternatively, these data may be due to simpler 

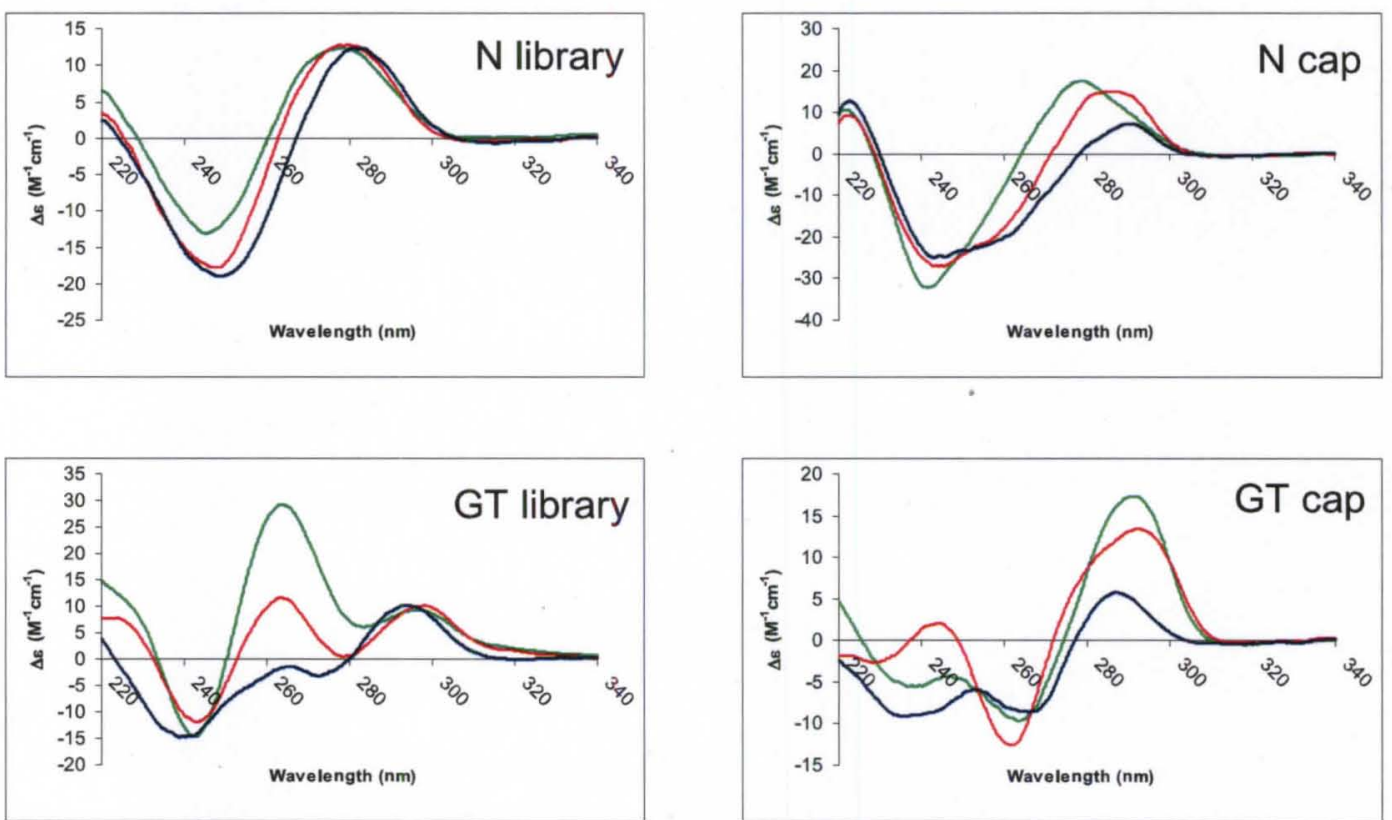

Figure 5. CD spectra of candidate aptamers and parent libraries. Green - $100 \mathrm{mM} \mathrm{KCl}$. Red - $100 \mathrm{mM} \mathrm{NaCl}$. Blue - water. Ncap and its parent library exhibit spectra suggestive of unstructured DNA. GTcap and its parent library exhibit spectra characteristic for G-quadruplex formation.

\section{Cell culture media PC3 extract}

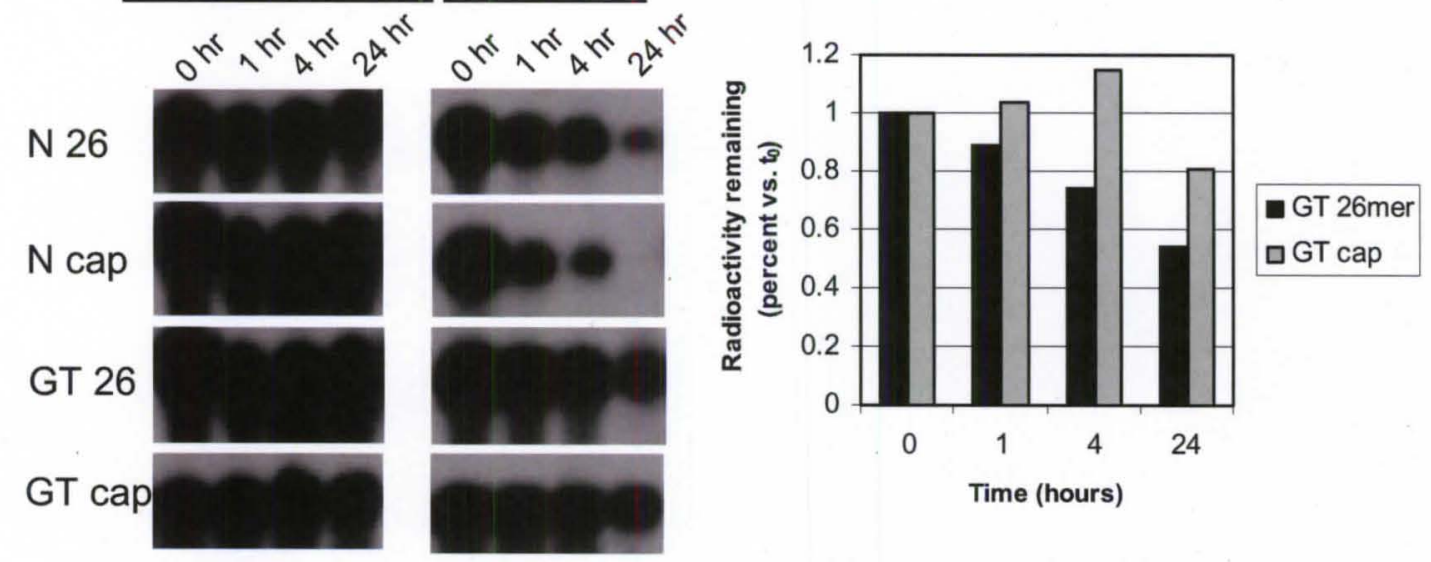

Figure 6. Stability of candidate aptamers and parent libraries. Endlabeled libraries incubated with either cell culture media containing $10 \%$ FBS or PC3 whole cell lysate for up to 24 hours. In the cell lysate samples, GT cap has modestly enhanced stability compared to parent library, while Ncap has decreased stability. Assay performed once. 
secondary structures such as hairpins or duplexes.

For the GT 26mer library, the CD spectra were similar to those previously seen (Chapter 2) and are in accordance with our previous conclusion that the GT library has a high proportion of molecules that adopt a G-quadruplex structure (a mixture of the "parallel" and "antiparallel" forms). We reach the same conclusion for the GT cap sequence, as the spectra exhibit peaks at the characteristic wavelengths for "antiparallel" G-quadruplexes and are most enhanced in the presence of potassium. The spectra of both the GT cap and GT 26mer library exhibit substantial polymorphism between the different solutions, again consistent with G-quadruplex formation.

\section{GT cap has modestly enhanced stability relative to parent library}

In our manuscript (Appendix A), we show that maximal degradation of our libraries was achieved before 24 hours with no further degradation seen as far out as 72 hours. We therefore tested the nuclease resistance of our candidates and their parent libraries for up to 24 hours $(0,1,4$, and $24 \mathrm{~h})$. We tested both complete cell culture medium (containing $10 \%$ heat-inactivated FBS) and $\mathrm{PC} 3$ cytoplasmic extract (Figure 6) in order to mimic the SELEX conditions in which the candidate aptamers were selected. With the cell culture medium, little degradation was seen for any of the samples. These data for the libraries are consistent with our previously published stability assay (Chapter 2). As the parent libraries themselves are largely stable under these conditions, we are unable show enhanced stability from our candidate aptamers. We do demonstrate that unlike some of our previously studied samples (e.g. AT 26mer, C 26mer) our candidates are not appreciably less stable than their parents under these conditions. 
In the PC3 extract samples, the $\mathrm{N}$ cap was clearly less stable than the parent library, and was fully degraded by 24 hours while the parent library persisted at that time point. Both the GT cap sequence and the GT library exhibited stability for all time points tested. Although it is difficult to demonstrate enhanced stability by our GT cap ODN because the parent library itself was largely stable even at 24 hours, densitometric analysis of the bands did indicate that the GT cap sequence was slightly more stable at 24 hours (81\% of $0 \mathrm{hr}$ versus $54 \%)$.

\section{Candidate aptamers have more complex protein binding than parent libraries}

Radiolabeled ODNs were incubated with PC3 whole cell extract and subjected to an electrophoretic mobility shift assay (EMSA) to study protein binding. AS1411 is included for comparison. Samples were analyzed alone (without protein) to examine labeling and molecular integrity (Figure 7). The labeling appears unequal across samples, and the protein binding data must accordingly be interpreted with caution. As before, we observed a difference in migration due to base composition, with the $\mathrm{N}$ library and $\mathrm{N}$ cap migrating faster than the G-rich samples.

In general, our candidate aptamers displayed more shifted bands than their parent libraries. We observe that the band marked " $\mathrm{B} 1$ " is present in all samples. A similar band was previously seen for both the N and GT libraries in four different cell lysates (Chapter 2). In our prior study we proposed that this band may represent a general nucleic acidbinding protein which may incur molecular stability, and these new data are consistent with that postulate. The less distinct band/region labeled as "B2" is perhaps very faintly visible in all samples, but is certainly most intense in the candidate aptamer samples. This 

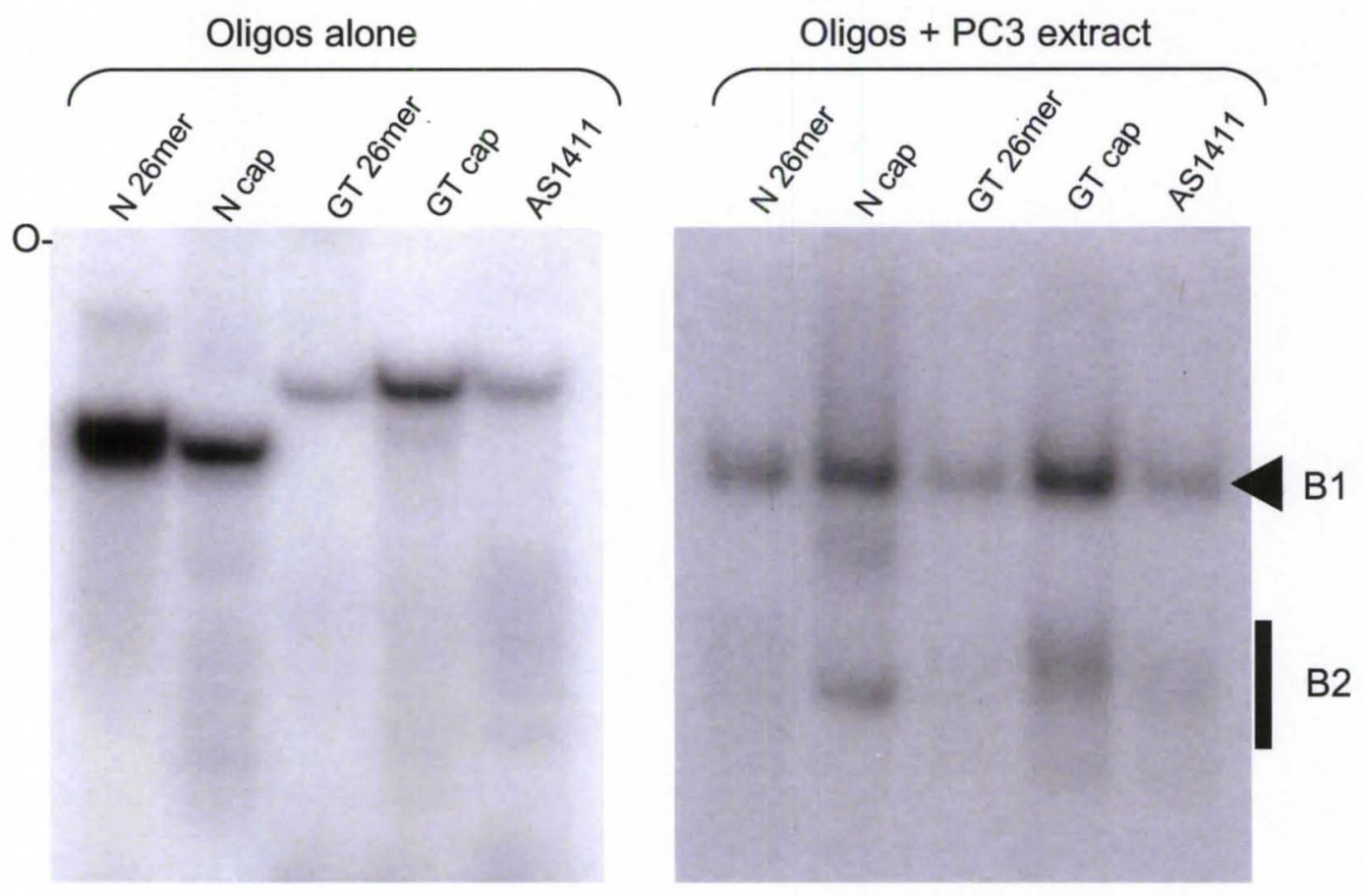

Figure 7: Protein binding of candidate aptamers and parent libraries in PC3 cytoplasmic extract. ODNs alone (with no protein) and with extract. ODNs alone remain intact from degradation, migrate at slightly different speeds, and exhibit some differences in labeling efficiency. Candidate aptamers have stronger and more complex patterns of protein binding than their parent libraries. AS1411 is included for comparison as a representative G-rich, G-quadruplexforming ODN. "O" denotes origin of the gel. 
may be due to the heterogeneity of the libraries (which are comprised of millions of possible sequences) versus the single species of each of the candidates; the libraries may contain only a fraction of molecules that bind the proteins seen in the B2 band whereas likely all the molecules in the candidate aptamer samples will bind.

As both the EMSA and the nuclease-resistance assay was performed in PC3 extract, and all ODNs were present at the earlier time points that would correlate to the time required to perform the EMSA, we are confident that the absence of shifted bands in a given sample as seen on EMSA is not due to ODN degradation. We conclude that our isolated candidates have more complex and more intense (though qualified by the difference in labeling efficiency across samples) protein binding in comparison with the originating libraries. In our previous work involving ODN libraries, we suggested that protein binding may be responsible for resistance to nucleases, and these data are consistent with our prior results.

\section{CONCLUSIONS}

Although we were initially gratified to have a single clear candidate from our cellSELEX using the $\mathrm{N}$ library after only five rounds of SELEX, we were unable to show enhanced uptake with the $\mathrm{N}$ cap sequence. Though we also examined molecular stability and antiproliferative activity, $\mathrm{N}$ cap did not display enhancement of these features. The $\mathrm{N}$ cap was eventually the only $26 \mathrm{mer}$ present in our sequencing samples, and we therefore conclude that the $\mathrm{N}$ cap is an artifact. In the time since we have performed these studies, newer literature reveals that a major shortcoming of SELEX is its vulnerability to amplification of artifactual error $(\underline{111}, \underline{130}, \underline{136}, \underline{137})$. If even a single molecule of a 
sequence that binds to an unintentional target is present, it will be amplified by the PCR reaction and will become enriched should the false target persist. Although not considered necessary at the time, many researchers now incubate the ODN library with the target in the presence of salmon sperm DNA, transfer RNA, and/or bovine serum albumin to block nonspecific binding. In addition to contamination with an unintended target, there are many other potential sources of artifact. One source in particular is the method that we employed to separate single- from double-stranded ODNs in the PCR product. Though the use of biotinylated primers and streptavidin beads was at the time believed to be sound, a subsequent report illustrated significant contamination of the "purified" PCR product by double-stranded DNA molecules, streptavidin, and biotin (137). The free streptavidin molecules were associated with the formation of cell and ODN aggregates. The researchers had previously identified aptamers with high binding affinity from a cellSELEX procedure, but when these candidates were ordered for verification and further testing, no binding was observed. They concluded that the SELEX procedure was confounded by the contamination, particularly the presence of streptavidin which, in addition to stimulating the formation of aggregates, may act as an unintentional protein target.

Another source of artifact particular to cell-SELEX is the presence of dead cells in the target sample. It is well known that dead cells are more likely to have permeable cell membranes, and it has been reported that free ODNs are fifty-fold more likely to associate with dead cells than live cells $(\underline{138)}$. Consistent with this, a recent study found that dead cells preferentially bound fluorescently-labeled ODNs during the SELEX process. Virtually all cells that stained with the viability marker 7-AAD were also 
strongly positive for ODNs. The authors report the failure in their hands of previous experiments (i.e. enrichment of sequences that did not exhibit enhanced binding) that neglected the issue of dead cells (136).

We attempted the SELEX method using a novel GT library and indeed, we were able to selectively evolve a candidate aptamer that had enhanced antiproliferative activity and nuclease resistance relative to the parent library. Consistent with our previous work using ODN libraries, the activity of the GT cap ODN may be dependent upon Gquadruplex formation, protein binding, and stability. However, despite our identification of the GT cap sequence, performing SELEX with the GT library was associated with a number of difficulties. The first was the problem of identifying candidate aptamers. Few ODN sequences were repeating by round 5 , and the artifact sequences (on the order of 400 bases in length) were increasingly prominent and likely depleting reagents in the PCR reactions. We were forced to resort to an unconventional method of motif-searching to find a candidate 26 mer. For libraries such as the GT, a pattern analysis software program to score sequences and motifs that are becoming enriched could provide some clarity. Discussions with the UL biostatistics department indicated that it is possible to design such a program for this specific purpose. We would test this program on our data set to see if it identified the same motifs/candidates that we identified by hand, and then use it on subsequent SELEX experiments (after addressing the issues of artifact).

Another problem unique to the GT library lies with the PCR reaction, a critical step of the SELEX protocol. G-rich ODNs are notoriously difficult to PCR, such that the GC content of primers and sequences is an important factor in designing the PCR thermocycler program. Variables such as the concentration of magnesium in the buffer 
must be adjusted in order to effectively amplify GC-rich stretches. In genotyping reactions, one group reports that the presence of G-quadruplexes and i-motif sequences in the DNA to be amplified consistently produces preferential amplification of one allele over the other resulting in error in diagnostic testing (133). Though we did not appreciate any differences in the quantity of artifact in the $\mathrm{N}$ versus GT SELEX, the high number of guanines in the GT library may have been a contributing factor. We used the same PCR program for both the $\mathrm{N}$ and GT SELEX, but the GT SELEX PCR could have been optimized independently. We expect this procedure would be cumbersome and costly in both time and funds as on the order of $25-50$ sequences would likely have to be cloned for each set of conditions tested. An alternate way to explore this issue is to perform SELEX as before with additional samples in which the libraries are applied to no target. These controls would help illustrate differences in PCR amplification of the N versus GT library.

Our SELEX protocol was intended to select for cellular uptake, operating under the widely accepted premise that increased uptake of our molecule would translate to increased activity. Recently published developments from Dr. E. M. Reyes-Reyes from our laboratory regarding the mechanism of action of AS1411 have called this basic premise into question (92). At early time points $(2 \mathrm{~h})$ the levels of uptake of AS1411 actually appears to be higher in benign cells than in cancerous cells, despite the fact that AS1411 has minimal effect on the growth of benign cells while strongly inhibiting the growth of cancer cells. Upon further investigation, it was discovered that initial uptake of AS1411 in the cancer cells (but not the nonmalignant cells) is by a unique endocytic mechanism called macropinocytosis. Macropinocytosis, as the name suggests, is a 
process by which cells ruffle the cell membrane and extend a relatively large appendage to engulf a sample of their immediate environment. By this endogenous method of uptake, cells are able to internalize large volumes of the surrounding media (along with any nearby molecules such viruses, bacteria, or drugs) in vacuoles termed macropinosomes $(\underline{139})$. Macropinocytosis has been shown to occur routinely in a variety of cell types, including epithelial cells, fibroblasts, and macrophages. At later time points in the cancer cells, AS1411 triggers hyperstimulation of macropinocytosis by a nucleolindependent mechanism. According to these new data, the absolute level of cellular uptake may be less important than the method of uptake by the cell. Therefore, in addition to technical improvements to the SELEX protocol, the selection of sequences that are able to stimulate macropinocytosis rather than merely internalized by cancer cells may be more apt in the study of G-rich ODNs. A preliminary study in that case would be to study the method of uptake of our ODN libraries (N 26mer, GT 26mer, GC 26mer) and to investigate which libraries, if any, are able to hyperstimulate macropinocytosis.

Despite many hurdles, with the utilization of the G-rich GT library we have identified a new anticancer aptamer with enhanced antiproliferative activity that is selective for cancer cells versus normal cells relative to its parent library. This aptamer also exhibited moderately enhanced nuclease resistance and complexity of protein binding than its parent library. The data from the GT cap sequence are consistent with our previous conclusions from studying ODN libraries, namely that the cancer-selective antiproliferative activity we have observed likely requires nuclease-resistance and binding to a specific protein or proteins. As others have also postulated, we believe that ODN stability may be necessary for antiproliferative cancer activity, but it is not 
sufficient in and of itself (그). Although GT cap does appear to form the G-quadruplex structure, we reiterate that the causal relationship between G-quadruplexes and antiproliferative activity is not yet determined.

There are now several biotechnology companies that are developing automated SELEX procedures (20) . As the technology improves, it may soon be possible to order aptamers against a particular target as one is currently able to order antibodies against an antigen of interest. In terms of commercial generation, SELEX has many advantages over antibodies. Not only are aptamers generated ex vivo with the associated simplification in standardization and purification, but the random SELEX starting library typically contains more different molecules by several orders of magnitude than a mouse possesses in its antibody spectrum (136). Screening of so many more different possible molecules may lead to greater specificity and selectivity in the evolved aptamers compared to antibodies. Alternatively, some reviewers suggest that it may eventually be possible to order commercial microfluidic kits for SELEX that would allow the rapid generation of target-specific aptamers in individual laboratories $(\underline{20}, \underline{140})$.

For these reasons and with consideration for the cost and effort that would be required for optimization in our hands, we chose not to repeat this project. However, one variation that we believe would be worthy of pursuit as a pilot study is in vivo SELEX. As whole cells are arguably a more clinically relevant target than purified proteins, aptamers selected against cancer cells in a true physiological environment may be the most applicable variation of SELEX in the development of new cancer drugs. To date, very few reports of in vivo SELEX exist. The majority involve the use of plants as the target $(\underline{141}, \underline{142})$. A single recent report has generated an RNA aptamer with selective 
uptake in liver metastases in a mouse model after fourteen rounds of SELEX (143). The target of this aptamer has been identified as $\mathrm{p} 68$, an intracellular protein that functions as an RNA helicase. We suggest an in vivo SELEX proof of principle protocol in mice as follows: implant subcutaneous DU145 prostate cancer tumors to athymic nude mice, allow tumors to grow until palpable, intravenously inject SELEX starting library (GT 26mer, N 26mer, or negative vehicle), euthanize animals after one hour, harvest tumors, homogenize the tissue, purify nucleic acid from the homogenate, and PCR. A new in vivo process such as this would likely require a great deal of optimization, but should it prove successful would comprise an early and significant contribution to the field of SELEX, aptamers, and potentially cancer therapeutics. 


\section{CHAPTER 5}

\section{EVIDENCE THAT AS1411 (A G-RICH APTAMER) INHIBITS THE GROWTH OF TUMOR INITIATING CELLS}

\section{INTRODUCTION}

\section{The cancer stem cell hypothesis}

Perhaps the most revolutionary new concept to impact clinical and experimental oncology in recent years is the cancer stem cell (CSC) hypothesis $(\underline{43}, \underline{44}, \underline{49}, \underline{50}, \underline{144})$. Though first proposed decades earlier, this hypothesis has truly risen to prominence since the mid-1990's. The central postulate is that a self-renewing subpopulation of cells within a cancer is responsible for its initiation and perpetuation. A popular representation of the CSC hypothesis is the "dandelion model": the malignancy is represented by a dandelion weed with the bulk of the tumor comprising the visible portion of the plant and the CSCs representing the hidden roots $(\underline{145}, \underline{146})$. Cutting off the weed at the surface is analogous to cancer therapies that merely debulk the tumor. According to the CSC hypothesis, if the CSCs are not targeted and destroyed, they will, with time, reestablish the weed of malignancy. In theory, this correlates with clinical relapse, which is all too commonly seen even after dramatic initial tumor regression. Conversely, a therapy that destroys the CSC roots beneath the surface may initially appear to have no effect on the weed, but eventually the dandelion would wither away. This is a stark contrast to both classic oncology goals of therapy and cancer research end points which include rapid reduction 
in tumor volume. Should the CSC hypothesis hold true, it will have (and is already having) fundamental effects on how cancer is studied and how new cancer therapies are developed.

It has been known for many years that cancer patients in remission routinely have cancer cells spread throughout their bodies, yet the vast majority of these cells will not proliferate $(\underline{147-150})$. This phenomenon suggests the possibility that while many cancer cells may be shed from the primary tumor, not every cancer cell is able to establish a metastasis. In animal studies, millions of cells from immortalized cancer cell lines are routinely injected per mouse in order to establish a xenograft, which implies that not all cancer cells within the injection are able to engraft. Although as of yet there are no reports showing that $\mathrm{CSCs}$ are directly responsible for relapse, a recent study of breast cancer patients have shown that following treatment, CSCs make up a higher percentage of the residual tumor population and correspond to a poorer prognosis (151). Another group reports a statistically significant relationship between the number of CSCs remaining in multiple myeloma patients following treatment and progression freesurvival (152). Though at this point such reports may be considered anecdotal, these articles contribute to a growing body of work that supports the CSC hypothesis.

\section{Clinical implications of the CSC hypothesis}

Current cancer treatments are able to decrease tumor burden and thus morbidity in patients. However, response to treatment does not necessarily translate into an increase in survival. It is well-known that although most patients with advanced cancer do initially respond to oncology treatment (typically a combination of surgery, chemotherapy, and/or radiation), the majority of these will relapse and eventually die from their disease, and the 
difference in time until death for responders and non-responders is often not statistically significant. Cancer types for which this has been shown include prostate, pancreas, lymphoma, and multiple myeloma $(\underline{152}, \underline{153})$. This phenomenon is in accordance with the CSC hypothesis. CSCs are believed to have many features that distinguish them from bulk tumor cells and make them intrinsically more resistant to classic cancer therapies, including those that target rapidly cycling cells, induce DNA damage, and are substrates for transmembrane efflux pumps. This means that current treatments may be effective only at mowing down the dandelion at surface level. Such efficacy against bulk tumor cells but not CSCs is the logical result of the standard cancer research models - for which decrease in tumor volume is a primary research endpoint - used to develop cancer therapies. According to the CSC hypothesis, if the CSCs are not destroyed they will divide, differentiate, and with time reestablish the malignancy. Worse, the treatment may have increased the proportion of cells that are resistant to treatment and/or more malignant. In prostate cancer it has been proposed that androgen ablation (castration) may stimulate a castration-resistant CSC compartment from quiescence to proliferation. This may trigger the development of a castration-resistant phenotype; that is to say, castration treatment of prostate cancer patients may actually hasten the progression to CRPC (154). To use the CSC analogy, castration is like denying the dandelion nutrition and causing it to wither only to stimulate the regrowth of a hardier, starvation-tolerant specimen.

Conversely, targeting the CSC subpopulation alone may also prove clinically insufficient depending on the lifespan of the non-CSC population. Although the terminally differentiated tumor cell population will, in theory, eventually senesce or 
apoptose, it is possible that the overall tumor burden may become clinically overwhelming to the patient before the tumor regresses. Destroying CSCs without taking steps to address the bulk tumor cells may thus result in unacceptable morbidity and mortality. Another possibility is that from among the genetically unstable cancer cells will evolve a new self-renewing population that can reestablish a CSC compartment. In all probability, leaving the bulk tumor cells untreated would increase the chance of such a mutation occurring.

Ultimately, cancer therapeutics will likely evolve into a multi-pronged, combinatory approach in which a cytotoxic agent will be combined with drugs that targets CSCs. This strategy theoretically will decrease the current tumor burden from bulk tumor cells as well as inhibiting regrowth from the stem-like subpopulation. A trial recently described for multiple myeloma patients studied the effects of a classic cytotoxic chemotherapy drug (cyclophosphamide) in combination with a drug aimed at destroying myeloma CSCs (rituximab). In addition to the significant relationship between CSC and progression-free survival mentioned above, the researchers found that the surviving CSCs had surface-bound rituximab, indicating that the drug was targeting the CSCs appropriately but was unable to destroy them in certain patients (152). This report strengthens support for the CSC hypothesis of cancer and encourages further study in the combinatorial therapy field.

\section{Theoretical ways to target cancer stem cells}

The most basic strategy in the treatment of cancer is to find the cancer cells and kill them, typically by identifying tumor-specific functions or antigens and exploiting 
them with targeted therapies. Destruction of the CSC subpopulation will likewise involve the identification of CSC-specific features. As CSCs are believed to have a unique stem cell-like biology, a variety of theoretical strategies to target and neutralize them take advantage of stem cell capabilities or requirements. Should such targets be found, a single anti-CSC therapy may potentially be effective against many different types of cancer.

One of the defining characteristics of CSCs is the ability to self-renew. It is this ability that allows the CSC compartment to maintain a malignancy. Therefore, one therapeutic strategy is to block self-renewal, forcing CSCs to either senesce or divide into two differentiated daughter cells with no self-renewal capabilities. With the loss of the self-renewing roots of the tumor, the cancer as a whole will eventually wither away. A possible complication is that if differentiation is induced, the ex-CSCs would join the pool of transit (or "transient") amplifying cells. Transit amplifying cells exist in a transitory state between stem cells and fully differentiated cells, and are characterized by a proliferative capacity that is more limited yet typically more rapid that stem cells. Thus, by forcing the differentiation of the entire CSC subpopulation, the global effect on the tumor could actually be accelerated growth. This effect should be self-limited, because with the loss of the CSC compartment, the terminally differentiated cells will eventually senesce. This is a situation in which combination therapy to control the overall tumor burden could be employed and keep this transient aggravation of tumor burden within clinically acceptable limits.

Another potential stem cell characteristic that may be exploited in the treatment of CSCs is the protected microenvironment known as the stem cell niche. The relationship between stem cells (benign or malignant) and their niches is complex and poorly 
elucidated, but it is certainly highly interactive. It is likely that the stem cells themselves contribute to the formation of their niche, and it is known that in normal, benign physiology the niche is critical to maintaining the stem cell phenotype. Disruption of the niche may mean that the CSCs can no longer survive or function as stem cells and could translate into loss of the CSC compartment. An alternate scenario is that an endogenous disruption in the regulation of the balance of the niche may have contributed to the formation of the cancer in the first place. In the prostate, it has been reported that stroma from prostate cancers may actually induce malignancy in previously benign epithelial cells (154). That is to say, the niche may not only protect CSCs, but may have caused them to develop in the first place. Correction of the aberrant biology of the niche may restore benignity to the stem cells within it. The interactions between the CSC and the niche is of great therapeutic interest, but should be studied with caution and rigor before interventions are attempted.

As the development of anticancer therapeutics specifically targeted against CSCs progresses, it will be important to minimize clinical side effects by sparing benign stem cells which share many markers, pathways, and characteristics (e.g. self-renewal and niche dependence) with their malignant counterparts. Anticancer therapy of any type should have high specificity for cancerous versus normal cells, but the stakes may be even higher when considering stem cells. Consider the hematopoietic stem cell; it would be wholly unacceptable to target leukemia stem cells only to cause life-threatening complications due to compromised immunity or hemostasis. However, in certain organ systems the distinction between CSC versus normal stem cell may be of little import depending upon the relative significance of the normal stem cell. As an example, breast 
cancer is most common in older women who have typically undergone menopause and have no particular need for the normal breast stem cell population. A therapy that targets breast CSCs could therefore also affect the normal breast stem cells with virtually no impact on the patient's quality of life. This also holds true for the prostate. Indeed, as benign prostatic hyperplasia (BPH) is a common medical condition that negatively impacts quality of life in the vast majority of the elderly male population, nonspecificity in targeting prostate stem cells could actually be beneficial even outside the field of oncology. If a PrCSC-targeting drug is easily tolerated, it could potentially also be used as a treatment for this notoriously inconvenient and widespread disease.

A final caveat is that the CSC compartment may comprise a "moving target" in clinical oncology; elimination of CSCs does not rule out the possibility that another CSC compartment may arise. One potential source for CSCs is the bulk tumor cells, which are notoriously genetically unstable and may undergo mutations that translate into dedifferentiation to achieve a more primitive phenotype. This could perhaps occur when subjected to unknown external stimuli from CSC-targeted therapies, similar to the natural selection of drug-resistant clones following chemotherapy. Also, the question of the cell of origin for cancer in general must be considered. It is possible that a CSC may arise from benign stem cells, transit amplifying cells, or fully differentiated somatic cells. A recent report from the University of Louisville describes a combination of genes that may be manipulated to convert benign mouse fibroblasts to a CSC-like phenotype (155). In leukemia, it has been shown that both hematopoietic stem cells and non-stem bone marrow cells may act as the cell of origin for cancer $(\underline{156}, \underline{157})$. Another group reported that a series of oncogenic insults converted benign prostate basal cells (which likely 
includes the stem cell compartment) to CSCs, but the same mutations applied to benign prostatic luminal cells were insufficient to create a similar effect $(\underline{158}, \underline{159})$. The researchers argue that in the prostate, one possible cell of origin for prostate cancer is the benign prostate stem cell. These studies provide insight regarding the fundamental question of how cancers develop. Although this question has yet to be answered, factors clearly exist that cause one person rather than another to develop cancer in the first place, and elimination of CSCs does not remove the potential for another CSC developing from other cells with the same environmental exposures and genetic risk factors.

\section{Cancer stem cells versus tumor initiating cells}

As discussed in the introduction (Chapter 1), the definitions, terminology, and methodology of the study of CSCs is far from standardized. For this body of work, we will utilize the American Association of Cancer Research definition of "cancer stem cell," whereby a CSC is characterized by the ability both to self-renew and to differentiate so as to establish and maintain a tumor. ( $\underline{50})$ The feature of self-renewal translates into the long-term survival of the CSC and therefore of the cancer. Asymmetric division allows the CSCs to proliferate into a diversity of cells that typically divide rapidly at the outset (as transit amplifying cells) and are ultimately responsible for clinical symptoms.

We observe that many reports involving the characterization of "CSCs" do not address the issue of self-renewal. We hold that the more appropriate term for this type of cell is "tumor initiating cell" (TIC). TIC refers most simply to a cancer cell type that has the capacity for tumorigenesis. Although this property implies the capacity for differentiation (i.e. the TIC can give rise to daughter cells that comprise the heterogenous 
bulk tumor population), TICs may or may not possess the ability to self-renew; in other words, CSCs are a subclass within the broader category of TICs. The general characterization of TICs is far from complete and sometimes hotly debated, but in addition to the defining characteristic of tumorigenesis, several features have come to be widely accepted. These include primitive, stem-like characteristics such as transient quiescence, membrane efflux pumps, and enhanced DNA repair mechanisms. Features such as these translate into resistance to classic anticancer treatments, and CSC/TICs have been strongly correlated to drug resistance and poor clinical outcome in a variety of settings $(\underline{64}, \underline{160-163})$.

One particularly interesting topic in the field of TICs is the discussion of the existence of different TIC subtypes. It is known that within the total cellular population of a given cancer, there exist subpopulations of cancer cells with different capacities for malignant characteristics such as tumorigenicity, proliferation, invasion, DNA repair, and drug resistance. In addition to a CSC class within the TIC compartment, perhaps enough heterogeneity exists such that some TICs are more tumorigenic than others or some are important in certain physiologic settings but not in others. For example, one subclass of TICs may be essential to the primary tumor while another variation of TIC is responsible for the formation of distant metastases. Information regarding TICs and variations within the TIC compartment may prove essential in the treatment of cancer.

Regardless of the precise definitions of CSCs versus TICs, the degree to which these cancer cell classes overlap, and their relative prominence in the total cancer cell populations, ultimately it is likely that complete cures from cancer will require specific targeting of unique subpopulations and their unique features. This makes the 
identification of pharmaceuticals that can target these cell types highly relevant in oncology research.

\section{Methods to study tumor initiating cells}

\section{$\underline{\text { Surface markers }}$}

The first and most common method to characterize TICs is by dividing cancer cells into subtypes based on cell surface markers. These markers sometimes have no known function, but may descriptively divide the cancer cell population until a signature is identified that is enriched in TICs in a process that is similar to the fractionation of an organic chemical solution (such as a plant extract) with anticancer activity. The chemical solution is divided into fractions that are tested for anticancer activity and the fraction with the highest activity identified. This fraction may then be subfractionated and the process repeated until in theory a single anticancer molecule may be identified. In the case of TICs, the criterion for fractionating cancer cells is tumorigenicity.

In our project, we investigate the following surface markers:

- $\mathrm{CD} 24 / \mathrm{CD} 44^{+}-\mathrm{CD} 44^{+}$primary prostate cancer cells have been shown to have higher proliferation, tumorigenicity, and metastatic potential than $\mathrm{CD}_{4} 4^{-}$cells. This feature also holds true in prostate cancer cell lines routinely used in cancer research (DU145, PC3 cells) $(\underline{164}, \underline{165})$. The $\mathrm{CD}^{-} 4^{-} / \mathrm{CD} 44^{+}$signature was originally described as enriched in CSCs for breast cancer (166). In the prostate, cancer cells with this signature have been shown to have higher soft agar colony formation, higher tumorigenicity in a limiting dilution nude mouse model, differentiation capacity in vivo, and gene expression patterns that are predictive of poor outcome (161). CD44 has also been reported as a predictive factor for 
prostate cancer recurrence ( $\underline{64})$.

- CD133 - a marker of stemness in many different benign and malignant cancer types, CD133 (in combination with CD44 and $\alpha 2 \beta 1$ integrin) was among the first markers described for prostate CSCs (167-169).

- Tumor-associated calcium signal transducer 2 (Trop2) - Also known as TACSTD2, M1S1, and GA733-1, Trop2 is a transmembrane protein of the EpCAM family with unknown function. Trop2 is enriched in prostate tissue samples after castration, in benign prostate spherocytes, and in the basal fraction of prostate epithelium. It has been reported as a marker for both prostate TICs and benign prostate stem cells $(\underline{170})$. It has also been suggested that Trop2 plays an active role in the tumorigenicity and invasion of prostate cancer.

\section{Functional markers}

- Side population

- Aldehyde dehydrogenase

Side population: ATP-binding cassettes (ABCs) comprise a large family of nucleotide-binding molecules, with on the order of 50 types in humans organized into seven families (ABCA to $\mathrm{ABCG}$ ). Most $\mathrm{ABCs}$ contain characteristic membrane-spanning domains and are believed to be involved in the transmembrane movement of small molecules. More specifically, they function by using energy from ATP to constitutively and non-specifically eject small molecules from the cytoplasm of cells. Particular ABC subtypes that are enriched in normal stem cells and CSCs include ABCG2 and ABCB1. (55) Prostate TICs have been reported to be enriched specifically in the ABCG2. 
One class of substrate for these pumps is small fluorescent dyes including Hoescht 33342 and rhodamine 123 (rho123). (171,172) These molecules freely diffuse across the plasma membrane, such that all cells will fluoresce by default when exposed to these dyes. However, cells that express high levels of these efflux pumps will remain dark. When subjected to flow cytometry, this population of unstained cells lie literally to the side of the main population and comprise the side population (SP). A SP, by definition, must disappear in the presence of a pump-inhibitor such as verapamil.

SPs have been shown to be enriched in TICs for many cancer types in both primary and established human cultures by a variety of methods $(\underline{173-179})$. Moreover, SPs are clinically relevant. One small study of clinical samples of ovarian cancer found that recurrent cancers were more likely to have a SP than primary cases, and that cancer cells harvested from ascites fluid or from peritoneal metastases (i.e. no longer localized) had a larger SP than cells from the primary tumor. More importantly, even with this small sample size, the authors report a statistically significant difference in prognosis for patients whose tumors had a SP versus non-SP cancer $(\underline{160})$. The authors add that a significantly greater percentage of cells lie within the SP for chemoresistant ovarian cancer cell lines versus chemosusceptible lines. The level of ABCG2 expression has also been reported as a predictor of poor outcome indicator for esophageal squamous cell carcinoma $(\underline{163})$. In neuroblastoma cell lines derived from patients at presentation and at relapse, the size of the SP was significantly increased for cells from the relapse samples $(\underline{162})$

Aldehyde dehydrogenase $(A L D H):$ ALDH is an intracellular enzyme highly expressed by stem-like cells. ALDH is believed to be a marker of TICs in many cancer 
types including liver, breast, and prostate. For prostate cancer, ALDH has been described as a marker for clonogenic, migratory, tumorigenic, and metastatic potential in both cell lines and primary human cells $(\underline{180}, \underline{181})$. Even more exciting, subtypes of ALDH have been reported as clinical prognostic indicators in breast $(\underline{182}, \underline{183})$, ovarian, $(\underline{184})$ and prostate cancer $(\underline{185})$. To measure ALDH activity, a commercial molecule called ALDEFLUOR which freely diffuses into cells and is a substrate for the ALDH enzyme may be used. Cells that express ALDH will cleave ALDEFLUOR, yielding a fluorescent product that can no longer diffuse across the cell membrane. Therefore, ALDH $^{\text {br }}$ cells (some of which presumably will be TICs) will fluoresce more brightly than $\mathrm{ALDH}^{-}$cells. As a negative control, samples containing cells and ALDEFLUOR are co-incubated with an ALDH inhibitor (DEAB). As with the side population assay, the $\mathrm{ALDH}^{\mathrm{br}}$ population is defined as those cells that are fluorescent with ALDEFLUOR, but not fluorescent with ALDEFLUOR plus the ALDH enzyme inhibitor.

\section{Sphere assays}

Among cancer cells that are typically cultured as monolayers, the vast majority require cell adhesion for survival. However, it is known that a subpopulation of cells will survive when grown in non-adherent culture conditions (with or without growth factors to stimulate stemness pathways such as bFGF and EGF). These cells may proliferate robustly under these conditions to form spheres (dense round clusters) of cancer cells up to on the order of a millimeter in diameter. Cancer spheres have been reported for many tissue types including breast (mammosphere), brain (neurosphere), and prostate (prostasphere, or prostatosphere) (186-189). It has been reported by many groups that 
sphere culture conditions enrich the total cell population for cells with stem-like features. The term spherocyte refers to a cell from a sphere. Spherocytes have been shown to have an increased proportion of TICs for many different cell types including both primary prostate cancers and prostate cancer cell lines (DU145, PC3) (190-192).

Different ways to utilize spheres in the study of TICs exist. As CSC/TICs are typically believed to comprise a small percentage of the total cancer cell population, some researchers culture spherocytes simply for the purpose of having a larger population of TICs to analyze. Another method is to plate cells in sphere culture to see if an experimental agent is able to block the formation of spheres. Still another is to allow spheres to form, and test different components or conditions to observe for dissolution of the spheres. The sphere culture model at this time is widely held to be the most applicable in vitro method in the study of TICs.

\section{$\underline{\text { In vivo tumorigenicity assays }}$}

In the identification of TICs, the gold standard is tumor growth of limiting dilutions of cancer cells in vivo; in theory every single cell of a stringently fractionated, pure TIC population will have the ability to establish a complete and heterogeneously populated tumor $(\underline{50})$. Researchers use dilutions of cells of different signatures (e.g. $10^{4}$,

$10^{3}, 10^{2}, 10^{1} \mathrm{CD}_{4} 4^{+}$cells versus $\mathrm{CD}^{-} 4^{-}$cells) and observe whether fewer cells of a particular signature are required for tumor growth. This implies that selecting for that signature enriches for CSCs.

For the study of CSC-targeting drugs, two main approaches have been described (193-195). The first is pretreatment of cancer cells with the experimental drug ex vivo 
followed by transplantation to mice. The rationale is that treatment will deplete the TIC subpopulation and that tumor growth will require a greater number of total cancer cells. This requires that the bulk tumor cells are not greatly affected by treatment with the experimental drug. The pretreatment approach may also employ the limiting dilution concept; pretreated and untreated cells may be implanted in varying numbers. The second approach is more powerful, involving establishing tumors in mice and then treating them with the experimental drug, alone or perhaps in combination with currently used chemotherapy. A TIC targeting drug should inhibit tumor growth, an effect that may be rapidly evident or may require a longer period of time in order for the bulk tumor cells to senesce and the tumor to regress. These models may be used in combination, and for this project we performed both the pretreated and long-term models.

\section{Rationale for this project}

Our hypothesis is that AS1411, a G-rich, quadruplex-forming aptamer that was studied in Phase II clinical trial as treatment for cancer, is effective at eliminating TICs. To test this, we used an established prostate cancer cell line and investigated whether AS1411 is capable of eliminating the TIC sub-population within these cancer cells. As discussed in Chapter 1, our hypothesis involving the effect of AS1411 on TICs arose from the clinical observation that treatment with the aptamer resulted in markedly delayed (rather than immediate) but durable tumor regression in a number of patients. A study of the scientific literature at the time revealed further correlates between aptamers and CSCs that suggest aptamers may be especially appropriate in the targeting of CSCs; aptamers may be more effective than other chemotherapy types at targeting quiescent 
cells, drug-resistant cells, cells able to repair DNA damage, and cells protected by their microenvironment (Chapter 1). Additionally, in our own laboratory, gene expression microarrays were performed on DU145 prostate cancer cells treated with AS1411 for 2 and 18 hours. This study found that, of the genes with the greatest difference in expression, a large number of those downregulated were implicated in stem cell biology. (unpublished data, Appendix C). This is consistent with our hypothesis.

Despite the uncertainties and controversial nature of the field, we undertook this high-risk project because the potential implications for aptamers, CSCs, and clinical oncology are great. Not only would this work shed light on the target of AS1411, but a clear corollary between AS1411 and CSCs could help elucidate the mechanism of action of AS1411, contribute to the rapidly growing knowledge of CSC biology, and further validate the field of aptamers, especially in the field of clinical oncology. It was decided that the possibility of high impact results validated the discovery-driven efforts that we describe in this chapter.

The question of studying primary prostate cancer samples versus established prostate cancer cell lines was considered. While primary specimens are clearly more representative of clinically relevant prostate cancer, much of the literature supports the concept of a TIC population existing (albeit in questionable percentages) in virtually all the established cancer cell lines studied. Many of the reports of TICs from a variety of cancer types employ cancer cell lines for their studies. For this preliminary project, we decided that use of established cancer cell lines is appropriate. As discussed in the previous section, DU145 TICs in particular have been relatively well characterized in vitro and in vivo. We therefore felt justified in utilizing this cell line for our experiments. 
We acknowledge the difficulty in definitively proving that a drug targets CSCs/TICs given the dearth of well-established methodology and rampant controversy in the field. For this reason, we employ a wide variety of methods and assays, each of which has been reported to distinguish TIC-targeting agents from non-TIC agents. While each assay will imperfectly overlap the TIC compartment, we propose that the accumulation of a large body of data exhibiting decrease in a purported TIC-enriched population in a multitude of ways will strengthen our hypothesis that AS1411 acts by targeting TICs.

\section{MATERIALS AND METHODS}

\section{Oligodeoxynucleotides}

Oligomers were purchased from Integrated DNA Technologies, Inc. (Coralville, IA) or from Invitrogen (Carlsbad, CA). Lyophilized oligomers were resuspended in filteredsterilized water, then heated at $65^{\circ} \mathrm{C}$ for 10 minutes and vortexed vigorously to dissolve. For in vivo studies, AS1411 was ordered in large-scale batches and resuspended in 10 $\mathrm{mM}$ potassium phosphate buffer $(\mathrm{pH} 7.4)$.

\section{Cells and monolayer culture conditions}

Three cell lines were studied: DU145 and PC3 prostate adenocarcinoma, and MCF7 breast adenocarcinoma. All cell lines were acquired from ATCC. Cells were grown in complete cell culture medium of DMEM (DU145 and MCF7) or F-12K (PC3) supplemented with $10 \%$ heat-inactivated FBS $\left(15\right.$ minutes at $\left.65^{\circ} \mathrm{C}\right)$ and $1 \%$ penicillin/streptomycin. The MCF7 cell line alone was grown with insulin $(0.01 \mathrm{mg} /$ $\mathrm{mL}$ ). All cells were cultured at $37^{\circ} \mathrm{C}$ in $5 \% \mathrm{CO}_{2}$. 


\section{MTT anti-proliferative assay}

AS1411 and the chemotherapy drug doxorubicin were tested in triplicate at a range of concentrations in DU145 prostate cancer cells for 72 hours. We modified previously published MTT [3-(4,5-Dimethylthiazol-2-yl)-2,5-diphenyltetrazolium bromide] assay protocol. Briefly, cells were seeded in 96-well tissue culture plates and incubated overnight. Cells were plated at 1000 cells per well. Drug stock solutions were then added to each well to yield the desired final concentration. Plates were incubated with the oligomer for three days, during which the cell culture medium was not changed. On day 3 , cell viability was determined and the background corresponding to medium alone (no cells) was subtracted. For each concentration, the average of the three wells was calculated and plotted.

\section{Stem cell marker staining}

Antibodies against human surface antigens along with coordinating isotype control antibodies were purchased from BD Biosciences (San Jose, CA) or Miltenyi Biotech (Auburn, CA). All antibodies were directly conjugated to fluorophores save the antibody against Trop2. For this antibody, appropriate secondary antibodies conjugated to fluorophores were used, and the secondary alone was used as the negative control.

For antibody staining of surface epitopes, cells were resuspended with TrypLE Express (GIBCO). The cell suspension was washed twice with cold staining buffer (1\% FBS in sterile PBS), then resuspended in $100 \mu \mathrm{L}$ of staining buffer at a final concentration of approximately $5 \times 10^{5}$ cells $/ \mathrm{mL}$. Appropriate antibodies were added directly to cells at concentrations recommended by the manufacturers. Samples were 
protected from light and incubated on ice $\left(4^{\circ} \mathrm{C}\right)$ for 30 minutes, and mixed occasionally by tapping during the incubation. Samples were then washed twice and resuspended in cold stain buffer for analysis. Samples requiring a secondary antibody were washed once incubated and with the secondary antibody for 30 minutes more, then washed and resuspended for analysis. Propidium iodide ( $2 \mu \mathrm{g}$ per sample) was added as a marker of viability. Cells were kept on ice and protected from light until analysis. Flow cytometry was performed using a FACScan flow cytometer.

For staining of internal stem cell markers, we used the BD Human and Mouse Pluripotent Stem Cell Analysis Kit with additional antibodies (Nanog, SOX 2) from BD Biosciences. We modified the manufacturer's protocol slightly as follows: up to $10^{6}$ cells were resuspended in $100 \mu \mathrm{L}$ of BD Fix buffer, then incubated at room temperature for 20 minutes. Cells were washed once with $1 \times$ Perm/Wash buffer, then resuspended in $100 \mu \mathrm{L}$ $1 \mathrm{x}$ Perm/Wash buffer and incubated for $10 \mathrm{~min}$ at room temperature. Appropriate antibodies were added directly to cells in stain buffer in volumes recommended by the manufacturer. Samples were protected from light and incubated at room temperature for 30 minutes. Samples were mixed occasionally by tapping during the incubation. After staining the samples were spun down, washed once with $1 \times$ Perm/Wash buffer, then resuspended in approximately $500 \mu \mathrm{L}$ stain buffer (1\% FBS). As we could not use propidium iodide as a marker of viability, we counted cells with Trypan blue before fixing and permeabilizing cells. Cells were protected from light until analysis. Flow cytometry was performed using a FACScan flow cytometer (BD Biosciences). 


\section{Side population assay (Rhodamine 123 dye exclusion assay)}

MCF7 breast cancer cells were grown to approximately $80 \%$ confluence in the appropriate cell culture medium, washed, and harvested with TrypLE Express. Cells were spun down and resuspended in prewarmed $\left(37^{\circ} \mathrm{C}\right)$ DMEM $+2 \%$ FBS +10 mM HEPES to make a final concentration of $10^{6}$ cells $/ \mathrm{mL}$. One to two $\mathrm{mL}$ of cell suspension were placed in darkened $15 \mathrm{~mL}$ tubes. The inhibitor verapamil was added to the control samples (10 $\mu \mathrm{M}$ final concentration) followed by $0.1 \mu \mathrm{g} / \mathrm{mL}$ of Rho1 23 added to each sample. Samples were placed in a $37^{\circ} \mathrm{C}$ water bath for 30 minutes with regular mixing by inversion of the tubes. Cells were spun down at $4^{\circ} \mathrm{C}$ and resuspended in $500 \mu \mathrm{L}$ ice cold HBSS $+2 \%$ FBS $+10 \mathrm{mM}$ HEPES. Propidium iodide $(2 \mu \mathrm{g}$ per sample $)$ was added as a marker of viability. Cells were kept on ice and protected from light until analysis. Flow cytometry was performed using a LSR II flow cytometer (BD Biosciences).

To study the effect of AS1411 treatment on the size of the side population (e.g. percentage of cells that remained unstained in treated versus untreated cells), MCF7 cells were treated with $10 \mu \mathrm{M}$ AS1411 or negative vehicle for 24 hours. Cells were washed, harvested, and stained with Rhol 23 as above.

\section{ALDEFLUOR assay}

The aldehyde dehydrogenase (ALDH)-positive population was assayed using a commercial kit (ALDEFLUOR, Stem Cell Technologies) according to manufacturer's directions. DU145 cells were grown to approximately $80 \%$ confluence in the appropriate cell culture medium, washed, and harvested with TrypLE non-trypsin enzyme (Invitrogen). Cells were spun down, then resuspended in room temperature Aldefluor 
Assay Buffer to make a final cell concentration of $10^{6}$ cells $/ \mathrm{mL}$. Aldefluor reagent was added to the cell suspension at a ratio of $5 \mu \mathrm{L}$ per $\mathrm{mL}$. Without delay, the sample was mixed and a portion of this sample added to a fresh tube containing the DEAB inhibitor. Another portion was placed in a fresh tube for staining with propidium iodide. Samples were incubated in $37^{\circ} \mathrm{C}$ water bath for 30 minutes, mixing occasionally by inversion. Cells were spun down and resuspended in ice-cold Assay Buffer to make a final concentration of $10^{6}$ cells $/ \mathrm{mL}$. Propidium iodide $(2 \mu \mathrm{g}$ per sample) was added as a marker of viability. Cells were kept on ice and protected from light until analysis. Flow cytometry was performed using a FACScan flow cytometer.

To study the effect of AS1411 treatment on the size of the $\mathrm{ALDH}^{\mathrm{br}}$ population (defined as cells negative for ALDH in the presence of ALDEFLUOR and DEAB, but positive with ALDEFLUOR alone) cells were treated with AS1411 at a range of concentrations and time points $(0-20 \mu \mathrm{M}$ AS1411 for 24-72 hours). Cells were washed, harvested, and stained with ALDEFLUOR as above. Experiments were performed thrice to ensure reproducibility.

\section{Soft agar colony formation}

DU145 cells were plated in soft agar as follows: a lower, cell-free layer of noble agar and cell culture medium $(0.6 \%$ agar and $70 \%$ complete cell culture medium final concentrations) was plated in $60 \mathrm{~mm}$ tissue culture plates and allowed to solidify at room temperature. DU145 cells were resuspended with trypsin. An upper layer of agar $(0.3 \%$ agar and $86 \%$ complete cell culture medium final concentrations) containing 2000 DU145 cells plus $10 \mu \mathrm{M}$ AS1411, $2 \mu \mathrm{M}$ doxorubicin, or no drug was plated over the 
solid lower layer. Plates were incubated at $37^{\circ} \mathrm{C}$ and $5 \% \mathrm{CO}_{2}$ for $10-18$ days until colonies grew to an appropriate size. Feeder layers of agar (including fresh drug) were applied weekly. Plates were stained with $500 \mu \mathrm{L}$ of MTT [3-(4,5-Dimethylthiazol-2-yl)2,5-diphenyltetrazolium bromide] $(1 \mathrm{mg} / \mathrm{mL})$ solution, then photographed and scanned (EPSO Expression 1680 scanner at $1000 \mathrm{dpi}$ ). Average colony size and total colony area for each sample was analyzed using Image-J software (National Institute of Health). Experiment was repeated thrice to ensure reproducibility.

\section{Prostate cancer sphere (prostasphere) culture}

DU145 prostate cancer cells were sorted for a subpopulation held to be enriched in TICs $\left(\mathrm{CD} 24^{\mathrm{lo}} / \mathrm{CD} 44^{\mathrm{hi}}\right)$, then grown in non-adherent conditions supplemented with growth factors to promote sphere formation and enrichment of stem-like cells. Serumfree sphere culture media was made fresh on the day of sorting consisting of: DME/F-12 (ThermoScientific), 1x B27 (Invitrogen), insulin ( $4 \mu \mathrm{g} / \mathrm{mL}$, Millipore), bFGF (20 ng/mL, Millipore), and EGF (20 ng/mL, Millipore). DU145 cells were stained for CD24 and CD 44 as described above, and the top $2.5 \%$ of $\mathrm{CD} 24^{\mathrm{lo}} / \mathrm{CD} 44^{\mathrm{hi}}$ cells were sorted at the University of Louisville cell sorting core facility using a MoFlo flow cytometer (Beckman Coulter). These cells were spun down, washed twice with $50 \%$ sphere culture media (diluted 1:1 with DMEM/F-12), and plated in T75 low-adherence flasks (Corning). The media was changed weekly. Cells were additionally supplemented with bFGF and EGF weekly.

To harvest spheres, the cell culture medium containing spheres in suspension was passed through a 70 micron nylon filter (BD Biosciences) to remove single cells and 
small cell clumps. Spheres were washed with PBS. The filter containing retained spheres was inverted over a clean 6-well dish and the spheres washed out with PBS. The spheres in PBS were spun down, resuspended in $1 \mathrm{~mL}$ TrypLE, and mixed by pipetting for 10-20 seconds to dissociate them into single cells. An equal volume of complete sphere media was added to neutralize the enzyme. Samples were counted with trypan blue; typical viability for primary spherocytes harvested and resuspended in this way was on the order of $80 \%$.

To study the effect of AS1411 treatment on spherocytes, DU145 CD24\% $/$ DD $44^{\text {hi }}$ cells were sorted and plated as described with the addition of $10 \mu \mathrm{M}$ AS1411 or negative vehicle for up to three weeks. For spheres grown in the presence of drug, fresh AS1411 was added at the time that the media was changed.

\section{Sphere survival assay}

To study the effect of AS1411 treatment on prostasphere survival, freshly sorted $\mathrm{CD} 24^{\mathrm{lo}} / \mathrm{CD} 44^{\mathrm{hi}}$ DU145 cells were plated in complete sphere culture media in 6-well ultra low attachment plates (Stem Cell Technologies, 2000 cells per well). Wells were treated with $10 \mu \mathrm{M}$ AS1411, $1 \mu \mathrm{M}$ doxorubicin, or negative vehicle. The media was changed weekly with fresh drug added at that time. Cells were additionally supplemented with bFGF and EGF weekly. Cells were cultured until an effect on sphere survival was seen, generally three weeks. Spheres were photographed in these plates. For quantification, the media and cells from each well were passed through a 70 micron nylon filter to remove any spheres/cells smaller than that diameter. Spheres washed into fresh wells with PBS and counted under the microscope. 


\section{Ex vivo pretreatment of cells followed by tumor growth in vivo}

This experiment was performed four times with slight variations in cell number and technique. Data are clearly marked with the variations. In general, DU145 prostate cancer cells were grown to approximately $50 \%$ confluence. Fresh medium was added, and AS1411 was added to a final concentration of $10 \mu \mathrm{M}$ versus negative vehicle. Cells were incubated for 72 hours. Cells were washed, trypsinized, and counted, then resuspended in PBS at a concentration of 1 or $2 \times 10^{6}$ per mL. Just prior to injection, the cells were mixed at a ratio of 1:1 with Matrigel (growth factor reduced, without phenol red (BD Biosciences)). Two hundred microliters of this suspension (containing $0.5-1 \times 10^{6}$ cells) were implanted subcutaneously to the right flank or the midline back of 5-8 female athymic nude mice (DU145 cells do not require androgen for growth, and therefore female mice may be used for in vivo experiments). Tumor size was monitored thrice weekly until animals were euthanized due to tumor burden. Tumor volume was determined by measuring the greatest linear dimensions in length and width (calculated by the equation $\left.\mathrm{V}=1 \times \mathrm{w}^{2} \times(\pi / 6)\right)$. The tumors were allowed to grow until tumor volume reached $10 \%$ of the total body mass of the animal, approximately $2 \mathrm{~cm}^{3}(2000$ $\mathrm{mm}^{3}$ ) for a 20 gram animal, at which point the animal was euthanized. Approval of this protocol was granted by the University of Louisville Institutional Animal Care and Use Committee.

\section{Long-term growth in vivo}

DU145 prostate cancer cells were grown to $90-100 \%$ confluence. Cells were washed, trypsinized, and counted, then resuspended in PBS at a concentration of $10^{7}$ per 
$\mathrm{mL}$. One hundred $\mu \mathrm{L}$ of this suspension (containing one million cells) were implanted subcutaneously to the right flank of twenty female athymic nude mice. Tumors were allowed to grow until palpable, then measured, and mice stratified into two equivalent groups.

The test group received intraperitoneal injections of AS1411 (10 mg/kg/day) in 10 $\mathrm{mM}$ potassium phosphate buffer $(\mathrm{pH}$ 7.4) while the control group received intraperitoneal injections of negative vehicle. Injections were given once daily for 21 days. Tumor size was monitored twice weekly from the start of the injections until animals were sacrificed due to tumor burden. Tumor volume was determined by measuring the greatest linear dimensions in height, width and length (calculated by the equation $\mathrm{V}=\mathrm{l} \times \mathrm{w} \times \mathrm{h} \times(\pi /$ 6)). The tumors were allowed to grow until tumor volume reached $10 \%$ of the total body mass of the animal, approximately $2 \mathrm{~cm}^{3}\left(2000 \mathrm{~mm}^{3}\right)$ for a $20 \mathrm{~g}$ animal, at which point the animal was euthanized. Approval of this protocol was granted by the University of Louisville Institutional Animal Care and Use Committee.

\section{RESULTS AND DISCUSSION}

\section{MTT assay used to assess test doses for AS1411 and doxorubicin}

The purpose of this project is to study the effect of AS1411 on TICs. As a negative control, we also tested the chemotherapy drug doxorubicin which is used as second line treatment for CRPC, widely used in other cancer types, and has been reported to have little effect on TICs (196-198). To minimize confounding effects on bulk tumor cells with doxorubicin treatment, we decided to select doses of doxorubicin that have only modest effects on the proliferation of DU145 cells for the timeframe we intended to 
study. In order to select an appropriate dose, we performed the MTT assay on DU145 cells at 72 hours using different concentrations of both AS1411 and doxorubicin (Figure 1).

In the majority of our TIC assays, the dose of AS1411 used is $10 \mu \mathrm{M}$. This dose was determined empirically, and was selected to illustrate the maximum effect of the aptamers on TICs. According to this MTT assay, at 72 hours AS1411 inhibits the growth of DU145 cells by about $30 \%$. From these data, we also decided upon the dose of $2 \mu \mathrm{M}$ doxorubicin, which inhibits the growth of DU145 cells by about $15 \%$. This range of doses is also supported by the literature in the study of CSC/TICs. $(\underline{193}, \underline{198-200})$ We decided that these doses of doxorubicin and AS1411 were comparable in terms of antiproliferative effect on bulk tumor cells, and could thus be used to illustrate differences in efficacy against TICs.

\section{AS1411 treatment induces cell cycle arrest but is not toxic to DU145 monolayer cells}

As a preliminary experiment to study the effects of AS1411 in our hands, we treated DU145 cells with 5 or $10 \mu \mathrm{M}$ AS1411 for 24 hours, prepared cells for flow cytometric analysis, and stained them with 7-AAD or propidium iodide. (Figure 2) We observed that AS1411 treatment results in modest accumulation of cells in the S phase of the cell cycle. (Figure 2A) This datum is consistent with previous reports $(\underline{34}, \underline{91})$. We also observed, again consistent with previous reports, that AS1411 causes only a minimal increase in cell death under these conditions. (Figure 2B) While previous studies have reported $\mathrm{GI}_{50}$ values of about $3 \mu \mathrm{M}$, those data were generated under different conditions, namely longer incubation times (6 versus 1 day) (그). This information generated under 


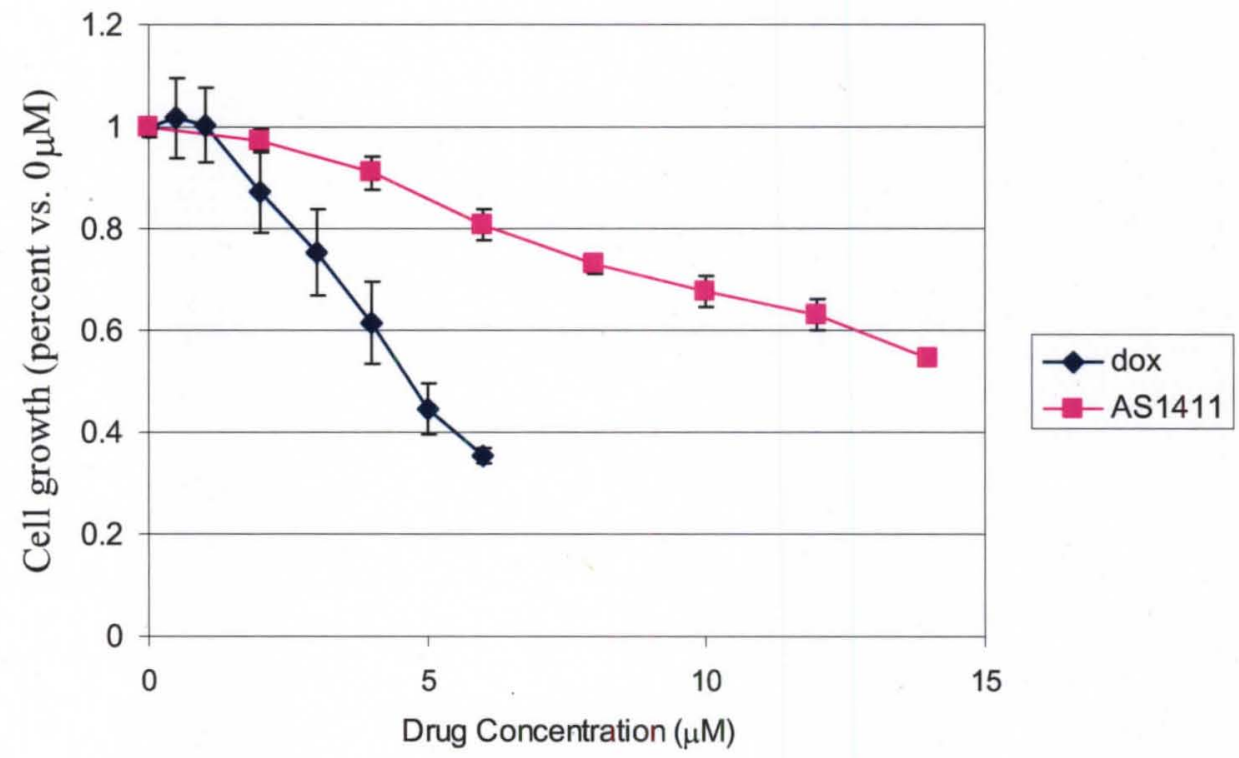

Figure 1: MTT assay of experimental drugs on DU145 cells after 72 hours 
A.

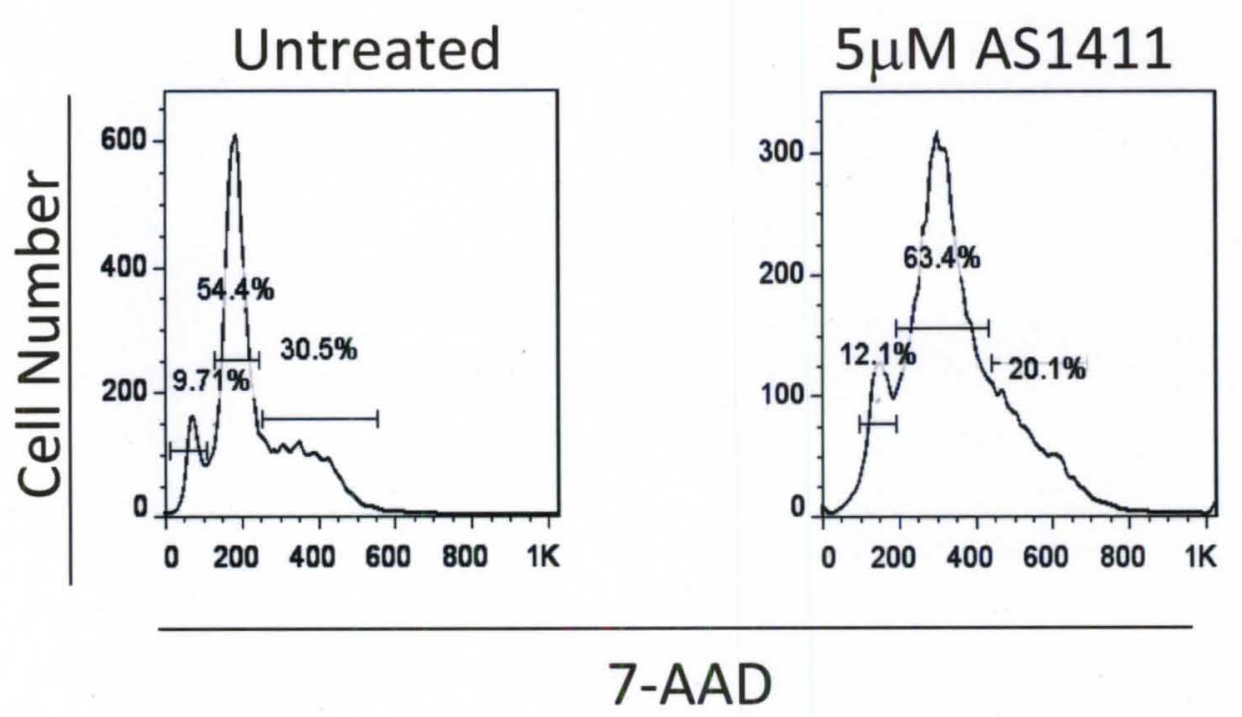

B.
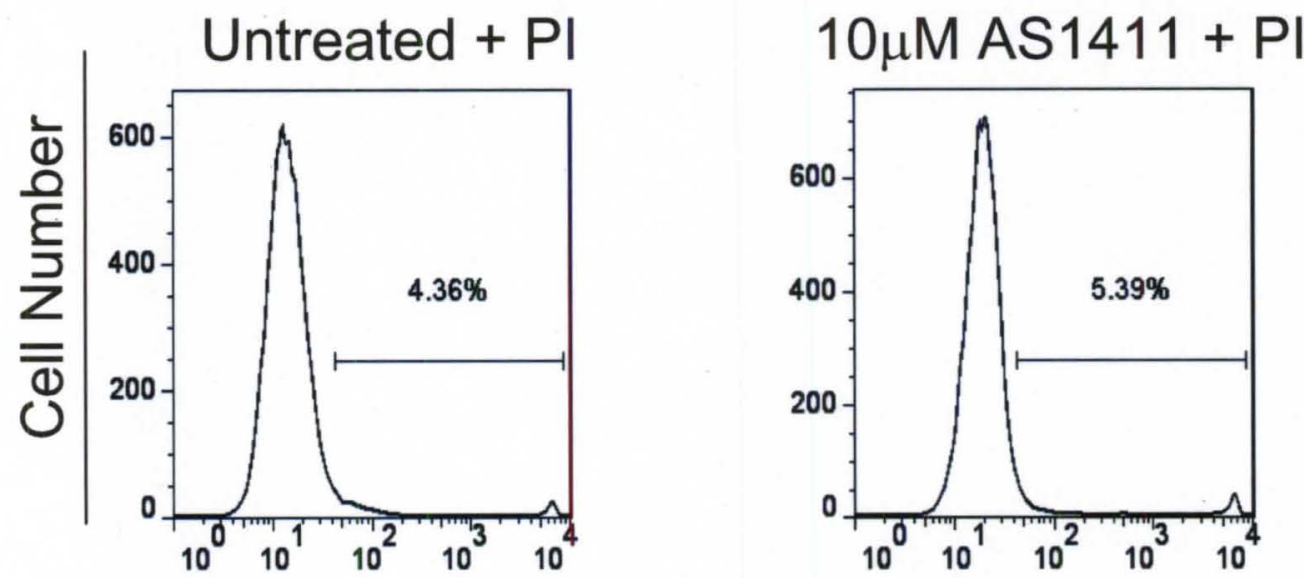

\section{Propidium lodide (PI)}

Figure 2: Cell cycle and cytotoxic effects of AS1411 on bulk DU145 cells.

A.Cell cycle analysis - 7-AAD.

B.Cell death analysis - propidium iodide 
our experimental conditions validates our methodology in preparing our samples, as well as aids in the interpretation of subsequent assays that study the effects of AS1411 on the TIC compartment. Because the bulk tumor cells are only marginally affected by AS1411 under these conditions, we can better determine whether or not any changes in cell growth and/or survival we see with our later assays are particularly targeting TICs. Though the data are reassuring, we did routinely stain samples with propidium iodide and/or trypan blue as a marker for viability for all subsequent experiments.

\section{CD24 ${ }^{\mathrm{lo}} / \mathrm{CD} 44^{\mathrm{hi}}$ sorted cells and spherocytes are enriched for prostate TIC markers}

As discussed previously in this chapter, $\mathrm{CD} 24^{\mathrm{lo}} / \mathrm{CD} 44^{\mathrm{hi}}$ prostate cancer cells are believed to overlap the TIC compartment. A similar argument has been made for prostate cancer cells that have been grown in non-adherent sphere culture conditions. To establish and validate the methodology in our hands, we analyzed three classes of DU145 cells: 1) unsorted monolayer cells, harvested by a trypsin analogue to make a single cell suspension ("monolayer" cells) 2) freshly sorted CD $24^{\mathrm{lo}} / \mathrm{CD} 44^{\mathrm{hi}}$ DU145 cells ("sorted" cells), and 3) cells from DU145 spheres that have been digested to form a single cell suspension ("spherocytes"). Our results are summarized in Figure 3.

We observed that a much greater percentage of sorted cells were CD24/ $/ \mathrm{CD} 44^{+}$ compared to unsorted cells $(86.0 \%$ versus $15.4 \%)$. As this is the cell surface marker signature for which we sorted, these data support the validity of our sorting procedure. We also observed that a greater number of sorted cells are $\mathrm{ALDH}^{\mathrm{br}}$ than seen in monolayer DU145 cells $(20.1 \%$ versus $7.51 \%, P<0.05)$. To our knowledge, this is a previously unpublished finding that provides additional evidence that prostate TICs are 


\begin{tabular}{|l|c|c|c|c|}
\cline { 2 - 5 } \multicolumn{1}{c|}{} & CD 24/44 & ALDH & Trop2 & Nanog \\
\hline Monolayer & $15.4 \pm 1.0 \%$ & $7.51 \pm 0.4 \%$ & - & - \\
\hline Sorted & $86.0 \%$ & $20.1 \pm 3.2 \%$ & - & - \\
\hline Spherocyte & $62.5 \pm 6.9 \%$ & N/A & $12.4 \%$ & $41.5 \pm 10.4 \%$ \\
\hline
\end{tabular}

Figure 3: Baseline levels of TIC markers in different types of DU145 cells. All cells analyzed in suspension by flow cytometry. Monolayer - unsorted DU145 cells. Sorted - CD24-/CD44hi. Spherocyte - cells from spheres grown from CD24/CD44 ${ }^{\text {hi }}$ cells in suspension culture. N/A - not assayable. (-) not detected.
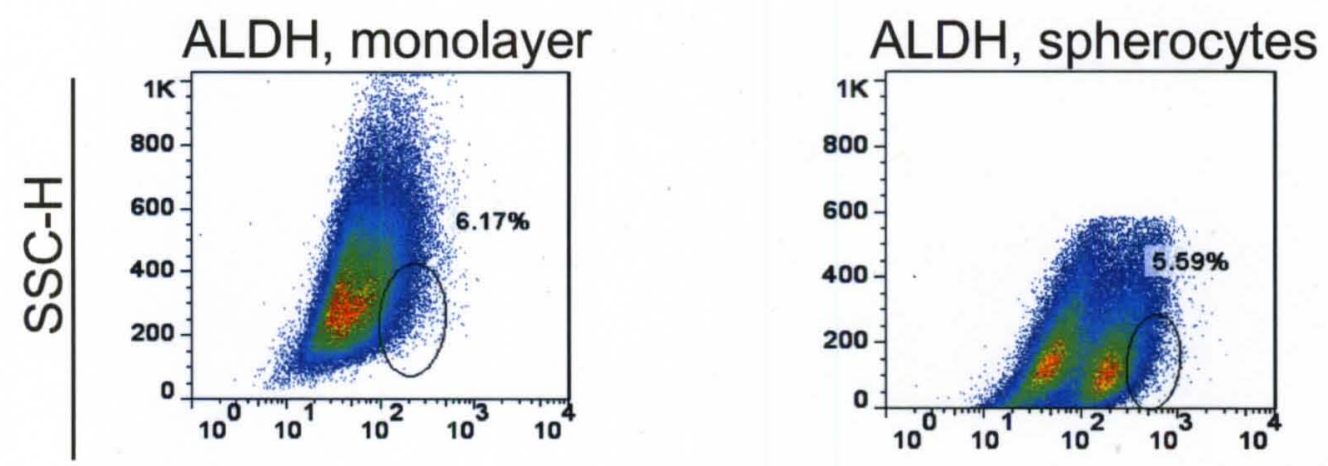

FL1-H

Figure 4: Heterogeneity of DU145 monolayer versus spherocytes cells 
contained within the $\mathrm{CD} 24^{\mathrm{lo}} / \mathrm{CD} 44^{\mathrm{hi}}$ compartment. As another assessment, we sorted the $\mathrm{CD} 24^{10} / \mathrm{CD} 44^{\text {lo }}$ double negative subpopulation at the same time as the $\mathrm{CD} 24^{-} / \mathrm{CD} 44^{+}$ cells. When stained with ALDEFLUOR, these double-negative cells had fewer ALDH ${ }^{\text {br }}$ cells (4.21\%) than not only the sorted cells, but also the unsorted monolayer cells. Though not statistically significant, this finding was not unexpected; if the CD24/CD44 ${ }^{+}$ subpopulation is enriched in stem-like cells, it follows that at least some other subpopulations are depleted. We studied both sorted and monolayer cells for several other reported TIC markers (Trop2, CD133, Nanog, Oct3/4, Sox2) but did not find detectable levels by flow cytometry.

Spherocytes were found to be enriched in $\mathrm{CD} 24^{-} / \mathrm{CD} 44^{+}$compared to monolayer cells $(62.5 \%$ versus $15.4 \%)$, but not compared to sorted cells. We reasoned that the sorted cells are most enriched in this surface marker signature because they are sorted based on these markers, and that once in sphere culture conditions some of the cells lose this signature via cell division and differentiation. We did find positive subpopulations of both Trop2 (12.4\%) and Nanog (41.5\%) in spherocytes versus undetectable levels in monolayer and sorted cells by flow cytometry. Taken together the data suggest that of the three prostate cancer cell types studied, it is the spherocytes that are most enriched in TICs. CD133 was once again undetectable.

Spherocytes were more challenging to assess due to limited viability and heterogeneity. The viability of freshly harvested spherocytes was typically around $80 \%$ as measured by trypan blue. Upon flow cytometric analysis, we observed a large degree of heterogeneity as seen by widely diffuse forward and side scatter clusters, and even clear subpopulations with different markers. For example, with ALDEFLUOR staining we 
observed two distinct groups of cells in spherocyte samples, while monolayer samples had a much more uniform population (Figure 4). We suggest that these phenomena are due to the physical structure of the prostasphere. Our spheres routinely grew to several hundred microns in diameter. It is therefore likely that cells embedded deep within the sphere are less able to receive nutrients, oxygen, and growth factors, and that cells in different positions within the sphere receive different growth signals that affect their protein expression. Because of these issues, we decided not to use the ALDH assay for these cells.

Our rationale for plating sorted cells was that a greater number of sorted cells would have the ability to form a sphere, and that we could perform our assays involving spheres more quickly by plating sorted cells rather than unsorted monolayer cells. The question has been raised whether or not unsorted DU145 cells would form spheres as robustly as those from sorted cells, and whether spheres grown from unsorted cells would show a similar enrichment of stem cell markers. We plan to undertake experiments to assess these questions in the near future. Nevertheless, these data show that, consistent with the literature, many markers for TICs are enriched in both the CD $24^{\text {lo }} / C D 44^{\text {hi }}$ subpopulation of DU145 cells and in cells grown in non-adherent sphere culture conditions. We therefore utilized the sphere culture method to assess the effect of AS1411 on the TIC subpopulation.

\section{Treatment with AS1411 decreases surface and internal markers believed to be associated with TICs}

Data from the functional markers (SP and ALDH) are discussed in separate 
sections below. We studied the effect of AS1411 on TICs by generating dose and time curves of on DU145 monolayer cells. Cells were treated at 24,48 , and 72 hours with 5 and $10 \mu \mathrm{M}$ AS1411, then stained for CD24 and CD44. The data are summarized in Figure 5. We quantified the percentage of DU145 cells that lay in the CD24 $/ \mathrm{CD}^{-} 4^{+}$quadrant as well as a subpopulation (about 5\%) that was negative for CD24 and brightest for CD44 $\left(\mathrm{CD} 24^{\mathrm{lo}} / \mathrm{CD} 44^{\mathrm{hi}}\right)$. The flow cytometry graphs for $10 \mu \mathrm{M}$ AS1411 at the three time points is shown in Figure 5A to illustrate the populations of interest. The smaller subpopulation demarked with a rectangle within the upper left quadrant is believed to be most enriched in TICs. In all cases, we found that AS1411 decreased the percentage of cells in both the whole quadrant of interest and the subpopulation (Figure 5B). The difference between untreated and treated samples was statistically significant, while the difference between the lower and higher dose was not. We concluded from this that the $5 \mu \mathrm{M}$ dose is sufficient to achieve maximal effect on the TICs at these time points. We repeated this experiment using lower doses of AS1411 (Figure 5C) at 72 hours and found that the decrease in these populations was in general dose dependent and can be seen with as little as $4 \mu \mathrm{M}$ AS1411.

Due to the resources and time required to generate prostaspheres, we limited our studies of the effect of AS1411 on prostaspheres to a single dose of $10 \mu \mathrm{M}$. To assess this effect, we developed an assay whereby freshly sorted CD $24^{\mathrm{lo}} / \mathrm{CD} 44^{\mathrm{hi}}$ cells were plated in sphere culture conditions with or without AS1411. Media was changed and fresh drug added weekly until any signs of senescence (less dense, less well-defined borders, more single cells) were seen. At this time all samples were harvested and analyzed. With this method, we found that AS1411 decreased the level of all measurable TIC markers: CD24- 
A.

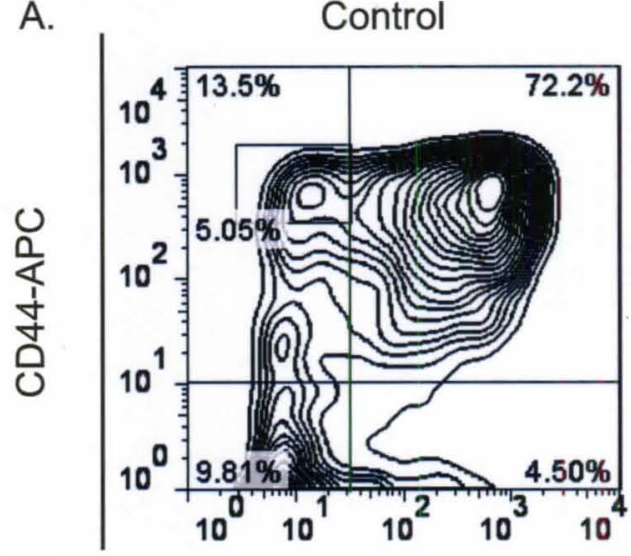

24 HOURS

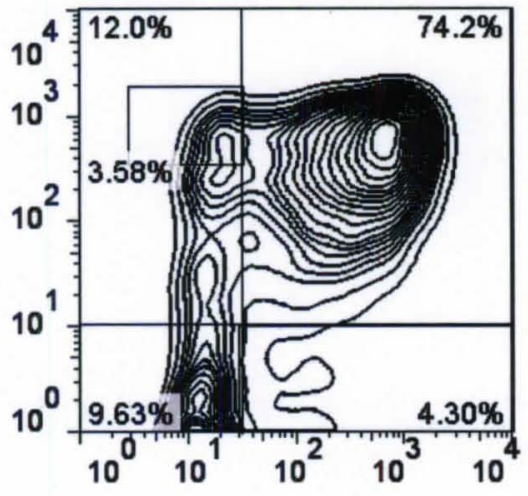

CD24-FITC

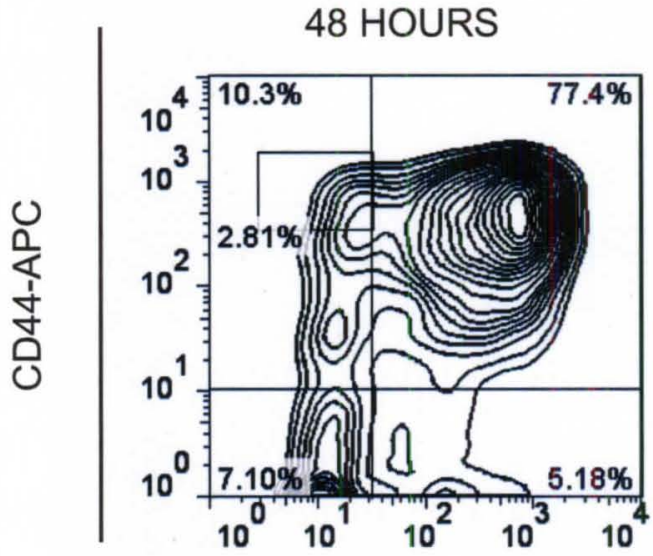

72 HOURS

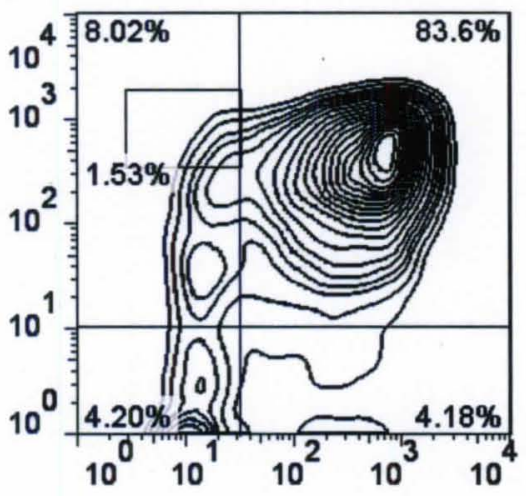

CD24-FITC

Figure 5: Effect AS1411 on percentage of CD24/CD44+ DU145 cells at various time points and concentrations.

A. Flow cytometry of unsorted DU145 monolayer cells treated with 10 $\mu \mathrm{M}$ AS1411 for up to $72 \mathrm{~h}$.

B. Summary of effect of 5 or $10 \mu \mathrm{M}$ AS1411 upon CD24-/CD44 ${ }^{+}$ subpopulation (upper left quadrant) and on CD24//CD44 ${ }^{\text {hi }}$ subpopulation (inset, upper left quadrant).

C. Summary of effect of $72 \mathrm{~h}$ treatment with 0-10 $\mu \mathrm{M}$ AS1411 upon CD24 /CD44 ${ }^{+}$and CD24/CD44 ${ }^{\text {hi }}$ subpopulations. 

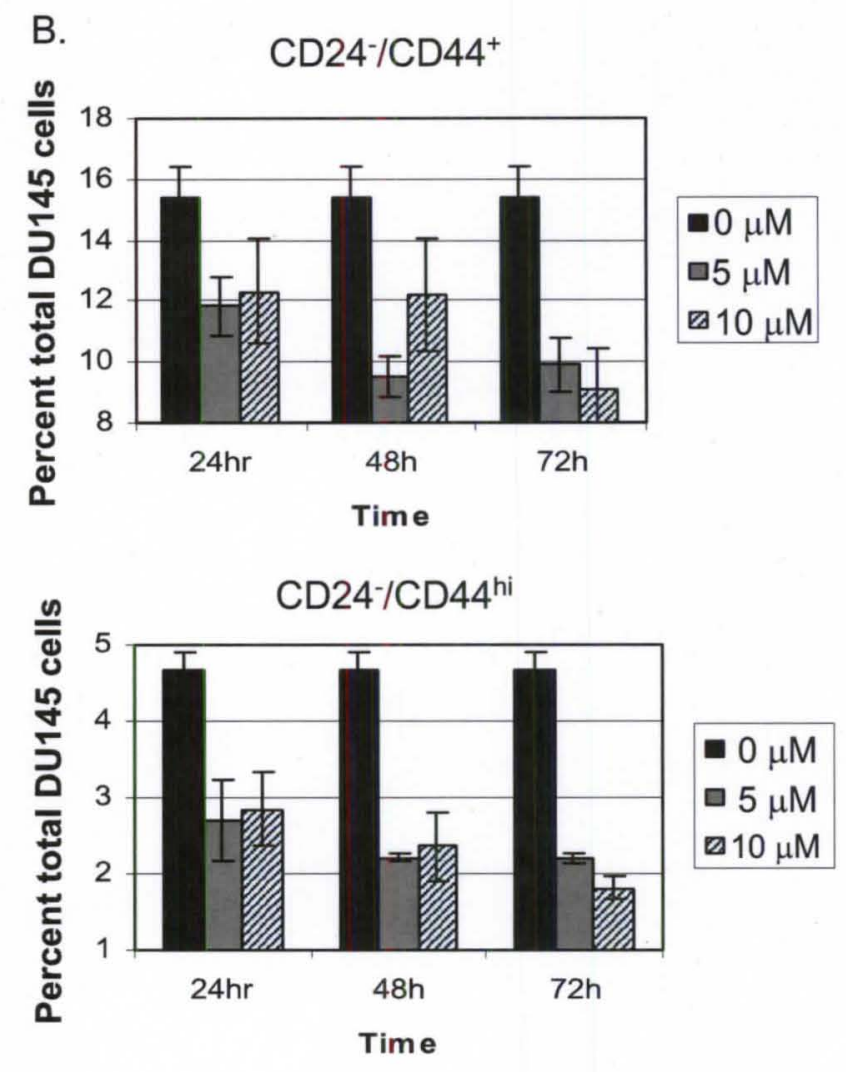

C. $\mathrm{CD} 24 / \mathrm{CD} 44^{+}$

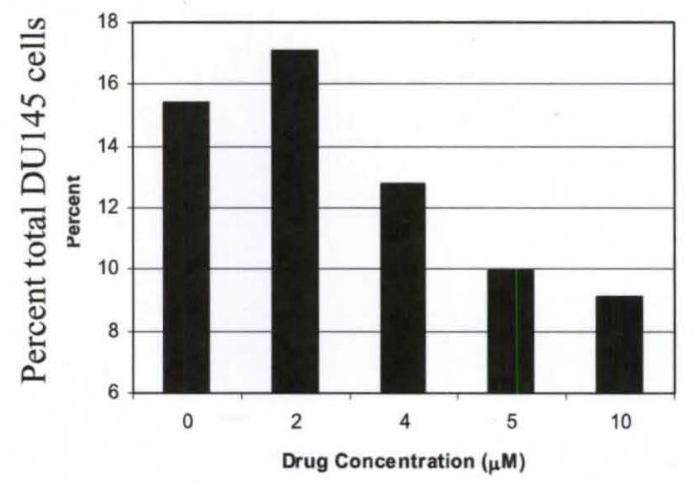

CD24/CD44

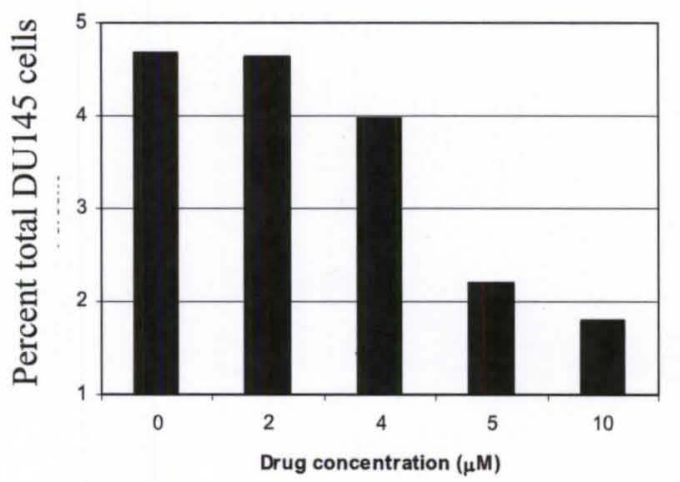

Figure 5, continued 
$/ \mathrm{CD} 44^{+}$, Trop2, and Nanog (Figure 6). Due to the time required for prostaspheres to mature, replicate experiments are ongoing. In addition, we plan to repeat certain key experiments with additional controls. We will repeat the treatment of DU145 monolayer cells for 72 hours and of spherocytes for approximately three weeks with $10 \mu \mathrm{M}$ AS1411 using doxorubicin as a non-TIC targeting control and a cytosine-rich analogue of AS1411 (CRO) as a control oligomer.

\section{Treatment with AS1411 eliminates the side population of breast cancer cells}

We attempted the SP assay using DU145 cells, but found that this cell line did not have a measurable side population either by Hoechst 33342 or by Rho123 staining. These data are consistent with reports from other researchers (201). We therefore employed the MCF7 breast cancer cell line to study the effect of AS1411 on this TIC-enriched subpopulation. Cells were treated with and without $10 \mu \mathrm{M}$ AS1411 for 24 hours, harvested and stained with Rho123. (Figure 7) Upon analysis, untreated MCF7 cells had a small SP $(0.406 \%)$ which did not stain with Rho123 and disappeared (i.e. became fluorescent) in the presence of the pump-inhibitor verapamil $(0.007 \% \mathrm{SP})$. MCF7 cells treated with AS1411 for 24 hours had virtually no cells in the side population $(0.004 \%)$. AS1411 had minimal effect on overall cell viability as measured by PI staining (data not shown).

As discussed previously in this chapter, SP cells have been shown to be enriched in TICs in a number of different cancer types. These data therefore support our hypothesis that AS1411 is an inhibitor of TICs. One caveat is that we cannot conclude from our data whether or not AS1411 is inhibiting the survival SP cells, and it is possible 

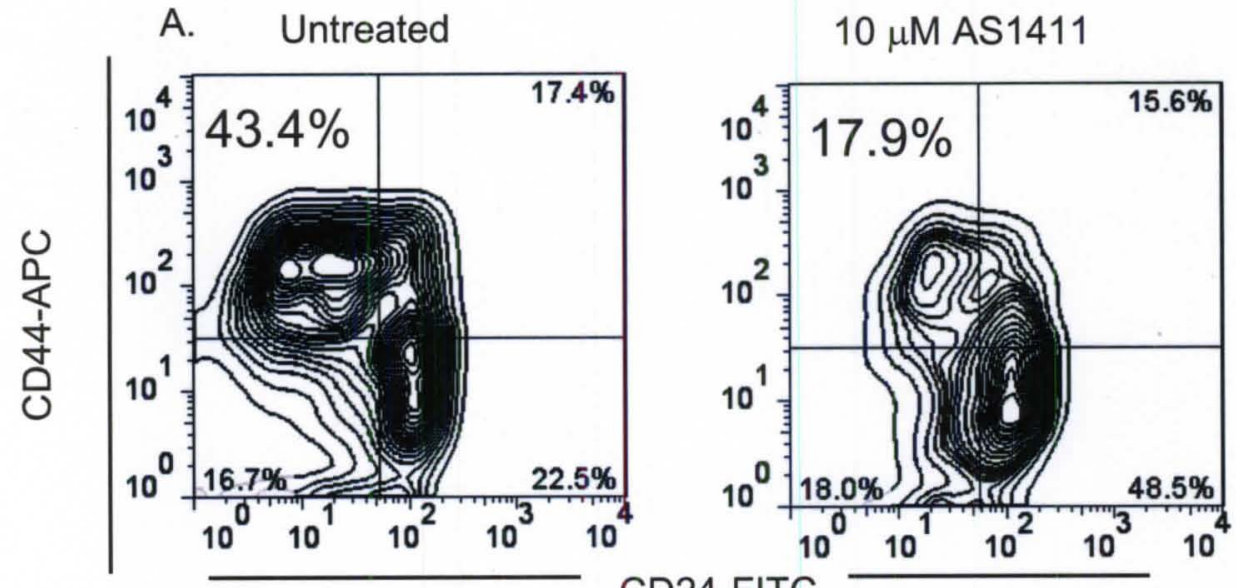

B.
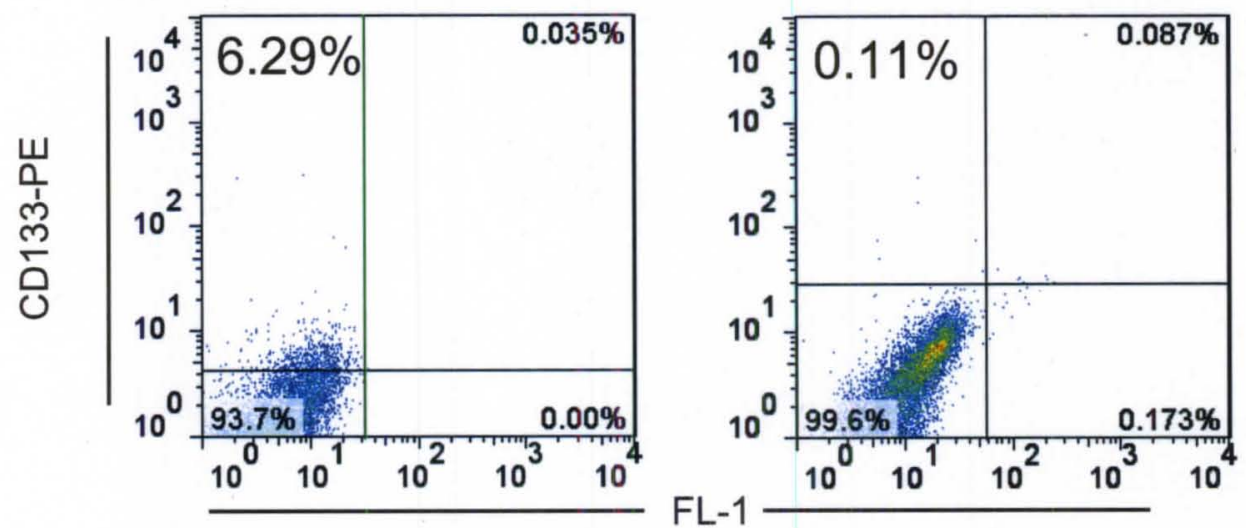

C.
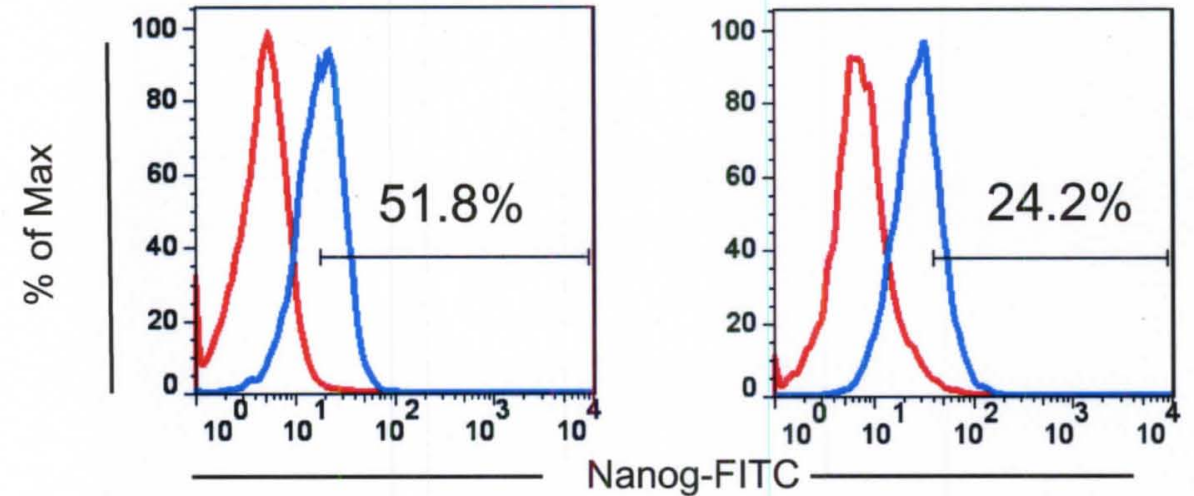

Figure 6: Effect of $10 \mu \mathrm{M}$ AS1411 on DU145 spherocytes, treated for 2-3 weeks in sphere culture.

A. CD24-/CD44+: $43.4 \%$ untreated, $17.9 \%$ treated.

B. CD133: $6.29 \%$ untreated, $0.11 \%$ treated

C. Nanog: $51.8 \%$ untreated, $24.2 \%$ treated 
Rhodamine123 Alone

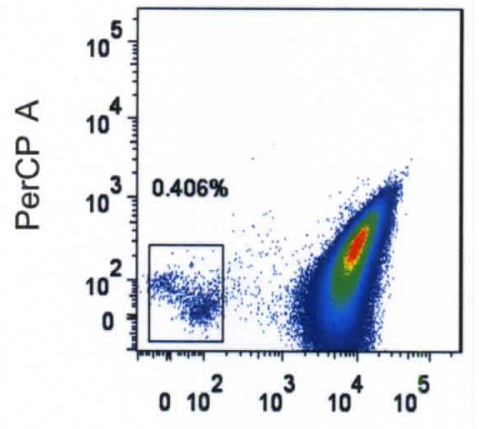

$0.406 \%$
Rhodamine123 + Verapamil Rhodamine123 + AS1411
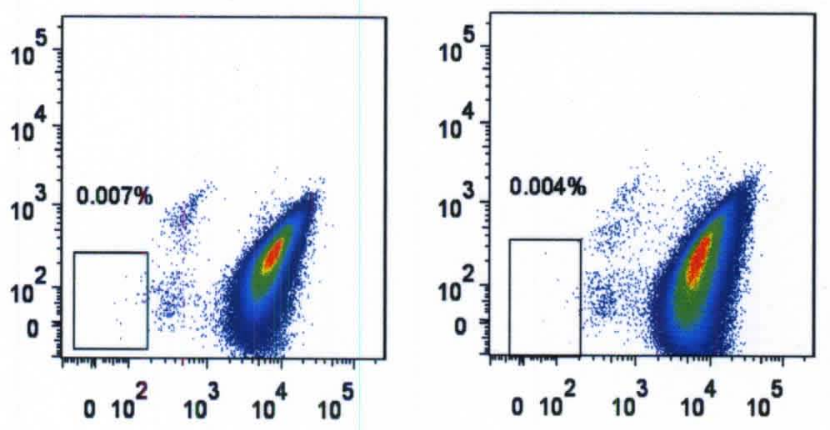

Rhodamine123 (FITC A)

$0.007 \%$

$0.004 \%$

Figure 7: Effect of $24 \mathrm{~h}$ treatment with $10 \mu \mathrm{M}$ AS1411 on Rho ${ }^{\text {neg }}$ side population MCF7 cells. 
that AS1411 is not frankly decreasing the number of cells in the population, but rather inhibiting the pumps that efflux the fluorescent dye. These pumps are known to act upon chemotherapeutic molecules of many types and have been implicated as the mechanism of drug resistance in cancer cell subpopulations $(\underline{52}, \underline{160}, \underline{163}, \underline{171})$. Therefore, if AS1411 acts by inhibiting the $\mathrm{ABC}$ pumps it could decrease drug resistance and potentiate the effects classic cytotoxic chemotherapies as combination therapy. Regardless of which case is true in actuality, we argue that both cases (decrease in cell survival and inhibition of ABC pumps) support the utility of AS1411 in the targeting of TICs.

\section{Treatment with AS1411 decreases the size of ALDH ${ }^{\text {br }}$ populations}

We assessed ALDH activity in DU145 prostate cancer cells treated with different doses of AS1411 or negative vehicle. (Figure 8) Untreated cells had a small and consistent $\mathrm{ALDH}^{\mathrm{br}}$ population of $7.51 \% \pm 0.81 \%$. Treatment with $10 \mu \mathrm{M}$ AS1411 for 24 hours decreased the $\mathrm{ALDH}^{\mathrm{br}}$ population to $1.34 \% \pm 0.68 \%(P<0.001)$. We also studied the effect of a smaller dose of AS1411. After 24 hours of treatment with $5 \mu \mathrm{M}$ AS1411, we found that the $\mathrm{ALDH}^{\mathrm{br}}$ population decreased to $0.43 \% \pm 0.29 \%$, a value even smaller than seen with $10 \mu \mathrm{M}$ AS1411. Although the difference between $5 \mu \mathrm{M}$ and control treated DU145 cells was statistically significant $(P<0.01)$, the difference between 5 and $10 \mu \mathrm{M}$ AS1411 was not. We conclude from this that $5 \mu \mathrm{M}$ at 24 hours is sufficient to significantly inhibit the $\mathrm{ALDH}^{\mathrm{br}}$ population, and that the higher dose of AS1411 has no additional effect. Unlike classic cytotoxic chemotherapies, for which higher doses translates into higher efficacy and doses are limited only by the patient's ability to tolerate the nonspecific toxicities, newer classes of targeted and biologically active 


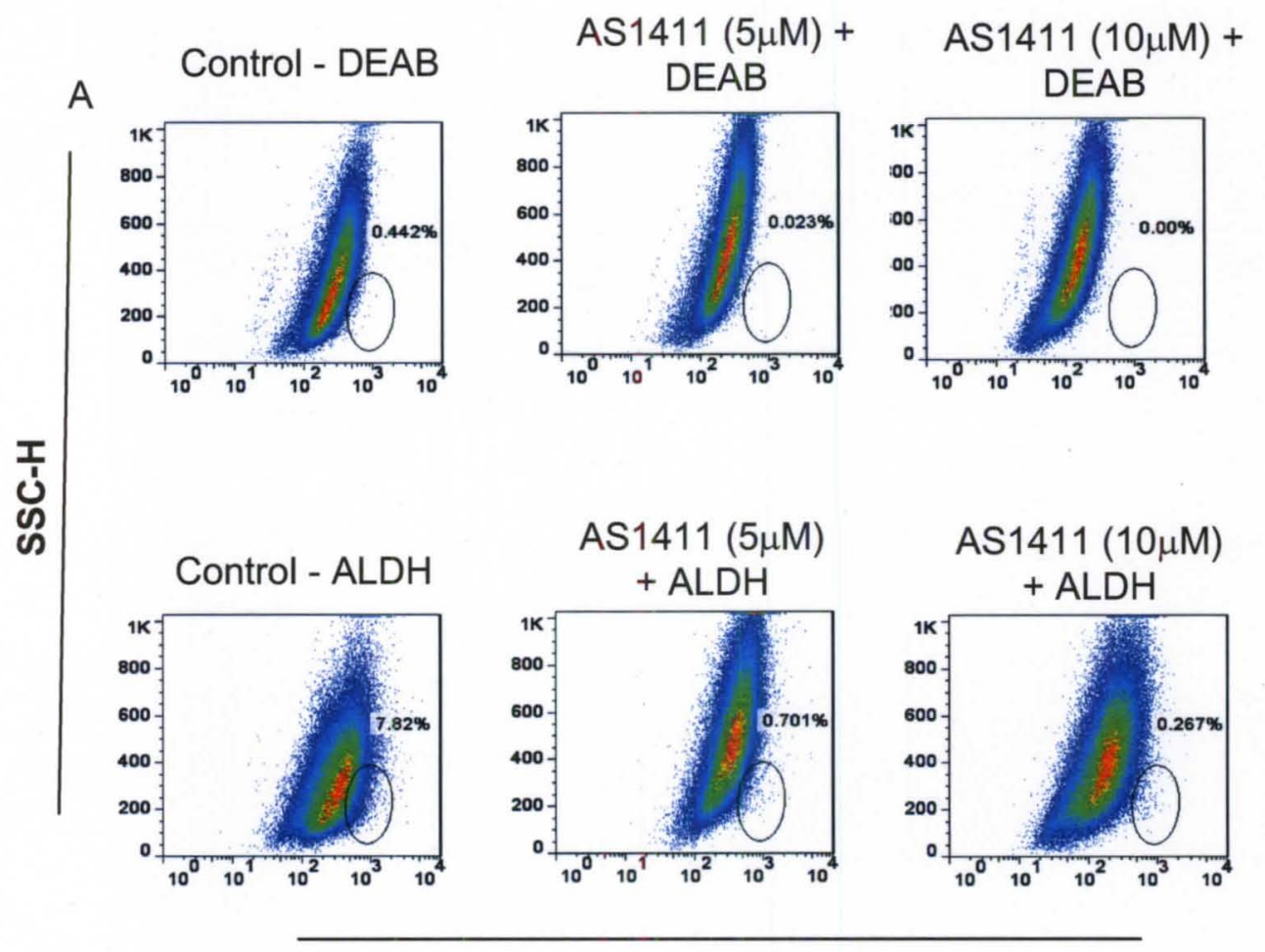

FL1-H

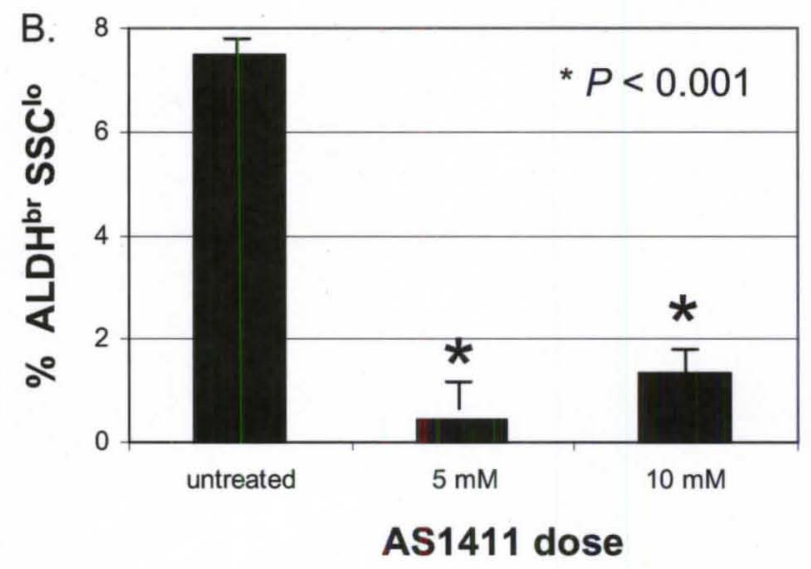

Figure 8: Effect of $24 \mathrm{~h}$ treatment with 5 or $10 \mu \mathrm{M}$ AS1411 on ALDH ${ }^{\text {br }}$ unsorted DU145 monolayer cells. 
chemotherapies may be most efficacious at moderate doses. Possible further experiments include the study of lower doses of $\operatorname{AS} 1411($ e.g. 1-5 $\mu \mathrm{M})$ for different time points.

\section{Treatment with AS1411 decreases the formation of DU145 colonies in soft agar}

Predating the emergence of the CSC field, the ability of cancer cells to proliferate and survive in soft agar culture is a classic model for tumorigenicity (202). We plated DU145 cells in soft agar with no treatment, AS1411, or the classic chemotherapy doxorubicin which has been reported not to inhibit the growth of TICs (196-198) . Cells were cultured for three weeks with new top layers of agar with cell culture medium and additional drug added weekly. (Figure 9A) Cells grown in the presence of AS1411 developed significantly fewer colonies than untreated cells $(366 \pm 92$ versus $1371 \pm 68$, $P<0.001$ ). (Figure 9B) The average size of colonies grown with AS1411 was also

significantly smaller than with no treatment $\left(0.23 \pm 0.03 \mathrm{~mm}^{2}\right.$ versus $1.00 \pm 0.17 \mathrm{~mm}^{2}$, $P<0.01$ ). (Figure 9C) Additionally, doxorubicin had a markedly smaller effect on colony number as compared to untreated cells (793 colonies $\pm 84, P<0.05)$. Colonies with doxorubicin and with no treatment were the same size $\left(1.00 \pm 0.13 \mathrm{~mm}^{2}\right)$ while the difference in colony size between AS1411 and doxorubicin was statistically significant, $(P<0.005$ compared to AS1411).

The soft agar assay has not yet been as clearly linked to the TIC subpopulation as other assays (such as sphere formation/survival). Despite this lack of association, this assay does illustrate the effect of treatment upon cells that do not require adhesion to matrix to proliferate. The soft agar assay may thus be considered as a functional marker for a more tumorigenic, invasive, and prone to metastasis cancer cell subpopulation. The 

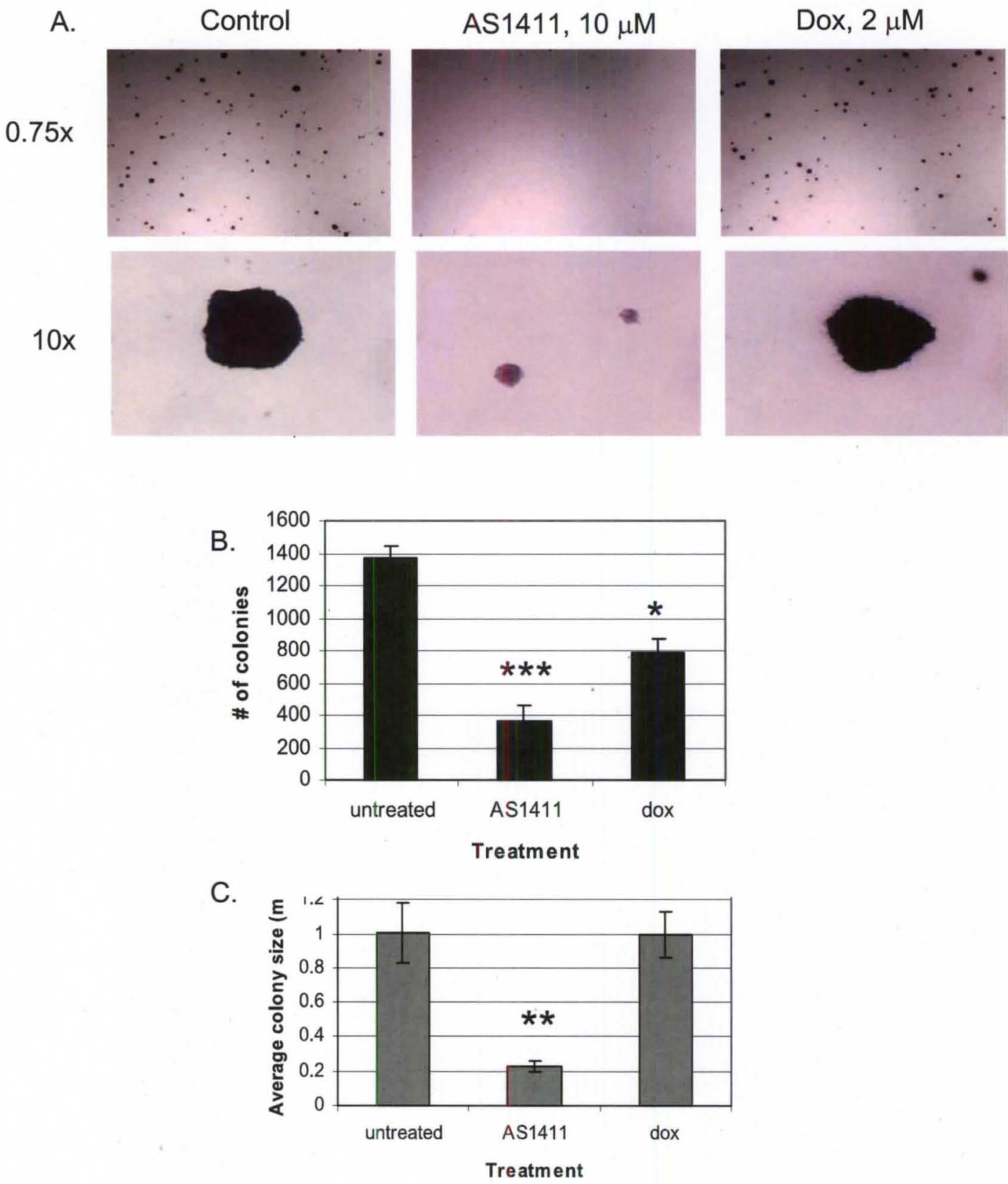

Figure 9: Soft agar colony formation of DU145 cells.

A. Light microscopy of colonies stained with MTT.

B. Analysis of colony number. Untreated vs. AS1411, ${ }^{* * *} P<0.001$.

Untreated vs. doxorubicin, ${ }^{*} P<0.05$.

C. Analysis of colony size. Untreated vs. AS1411, ${ }^{* \star} P<0.01$. AS1411 vs. doxorubicin, ${ }^{* *} P<0.01$. 
data we have generated, that AS1411 strongly inhibits the growth of colonies in soft agar while doxorubicin modestly inhibits their growth, is therefore noteworthy and supports our hypothesis that AS1411 is targeting a TIC compartment.

\section{Treatment with AS1411 inhibits prostasphere survival}

Freshly sorted CD $24^{\mathrm{lo}} / \mathrm{CD} 44^{\mathrm{hi}}$ DU145 cells were plated in sphere culture media with no treatment, AS1411, or doxorubicin. At the end of the experiment, all spheres greater than 70 microns were counted for each sample. (Figure 10) We found a decrease in the number of spheres that survived AS1411 treatment versus no treatment $(5.7 \pm 4.3$ versus $42.7 \pm 12.3$ spheres, respectively per 2000 cells plated). Even more exciting, as with the soft agar assay we found that doxorubicin had a much more attenuated effect on sphere survival ( $34.7 \pm 14.8$ spheres). These data approached statistical significance, but additional replicates with our refined sphere culture techniques will be required.

Other putative TIC-targeting drugs have been reported to inhibit sphere formation $(\underline{193}, 194)$. We observe that AS1411 did not inhibit the formation of cancer spheres, but rather promoted their demise. This is in contrast to the soft agar assay where colonies are inhibited from forming by AS1411 treatment. A decrease in sphere survival may more accurately model the common clinical scenario in which an existing tumor must be treated, i.e. a newly diagnosed cancer patient with frank disease. As CSCs are a subclass of TICs, so other subclasses may exist, such as primary TICs that are responsible for the growth and maintenance of the primary tumor and metastatic TICs that are responsible for tumor growth at distant sites. It is possible that these two assays (colony formation and sphere survival) distinguish different types of TICs. It may be that different classes of 
A.

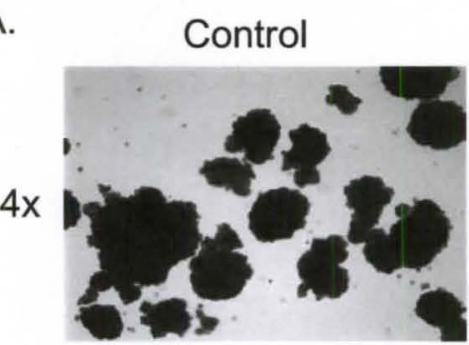

AS1411, $10 \mu \mathrm{M}$
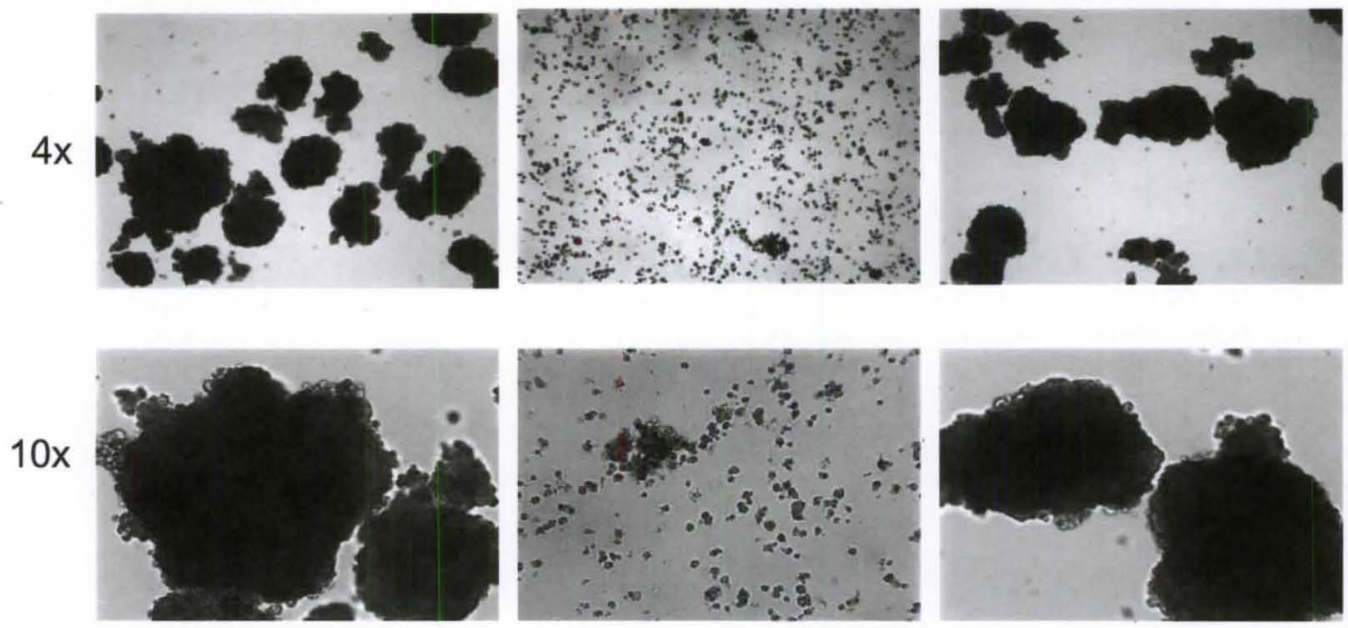

B. 60

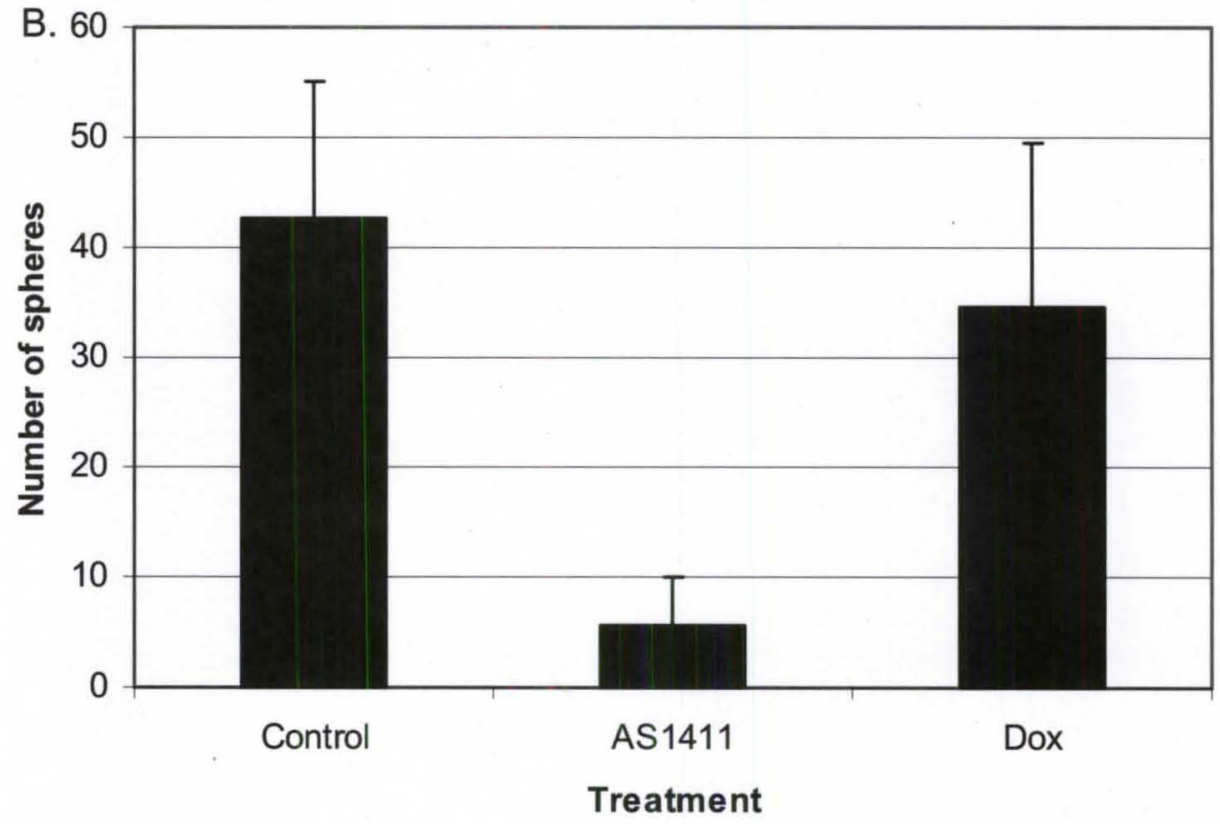

Figure 10: Sphere survival assay.

A. Light microscopy.

B. Total number of spheres retained after passing through 70 micron filter. 
TIC-targeting drugs will ultimately prove necessary to both cure the initial malignancy and prevent recurrence. AS1411 appears to be effective against both the malignant subpopulation that can grow in soft agar and the subpopulation that can maintain a prostate cancer sphere.

For this assay we used $1 \mu \mathrm{M}$ doxorubicin. When this assay was performed we had not yet assessed the effect of doxorubicin on the bulk tumor cells. As discussed previously, a higher dose $(2 \mu \mathrm{M})$ would give us a fairer comparison with this dose of AS1411. We plan to repeat this assay in order to achieve statistical significance. At that time we plan to study the higher dose of doxorubicin. We also plan to test oligomer libraries and candidate anti-prostate cancer aptamers from our previous work: N 26mer, $\mathrm{N}$ cap, GT 26mer, GT cap, GA 26mer, GC 26mer, and a C-rich analogue of AS1411 in which all guanines are replaced by cytosines (CRO: 5'- CCT CCT CCT CCT TCT CCT СCT СCT CC -3 ').

\section{Pretreatment of cancer cells with AS1411 before implantation to mice results in slowed or delayed tumor growth}

We performed an in vivo experiment in which DU145 prostate cancer cells were treated ex vivo for 72 hours with AS1411, washed, and equal numbers of viable cells implanted subcutaneously to nude mice. This pretreatment model has been described in a number of different reports that study the potential TIC-targeting ability of pharmaceuticals (193-195). While certainly less clinically pertinent than the treatment of established tumors with long term follow up, we undertook this method to see if the molecular results we have generated thus far in vitro would translate into an effect in the 
complexity of the murine physiologic environment. In essence, the mouse acts as a more physiologically relevant cell culture system. We felt comfortable employing this model because it is known that cells treated with AS1411 for up to $72-96$ hours will recover if the drug is removed ( $\underline{34})$.

The tumor growth curves from the pretreatment model experiments are shown in Figure 11. We performed this experiment four times, and each time experienced technical difficulties with the injections which resulted in different artifacts in tumor growth for some mice. A number of mice of the first two experiments developed tumors that grew within the peritoneal cavity rather than subcutaneously (Figure 11A and B). Some of the mice of the second experiment developed ascites, likely due to inadvertent direct injection of tumor cells into the liver (Figure 11C). We then switched the location of the injections to the skin of the midline back (Figure 11D) which avoided the abdominal cavity altogether, but found that the shapes of the tumors were much more irregular; several mice had very elongated and narrow tumors, while others grew two distinct masses instead of one.

For analysis, we eliminated data for any mouse with tumor growth that was clearly not limited to the subcutaneous area as we reasoned there must be tumor present that was not measurable by calipers. Because of these issues, the experiments finished with fewer mice than we intended and we did not achieve the significance that we hoped. However, for every data point that was statistically significant, tumors from the pretreated cells were smaller than the control treated cells (marked with asterisks), and in general all the curves indicate that pretreatment with AS1411 inhibits the growth of tumor, either by blocking tumor take or slowing tumor growth. We conclude that 
A. $10^{6}$ cells, flank

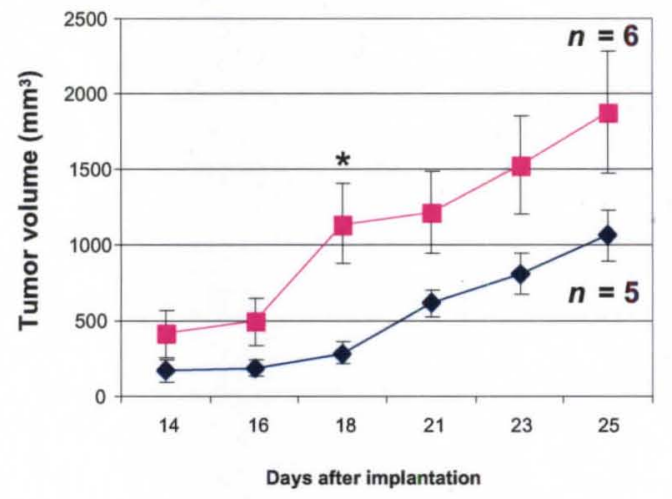

C. $5 \times 10^{5}$ cells, flank

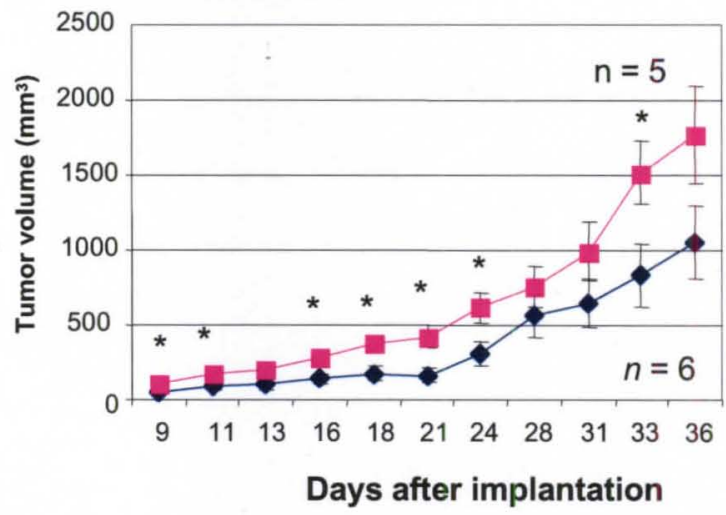

Control
Pretreated (10 $\mu \mathrm{M}$ AS 1411)
$P<0.05$
B. $2.5 \times 10^{5}$ cells, flank

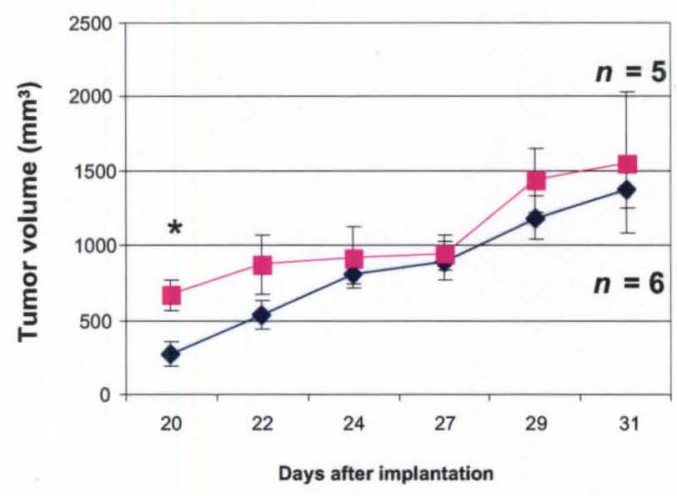

D. $5 \times 10^{5}$ cells, back

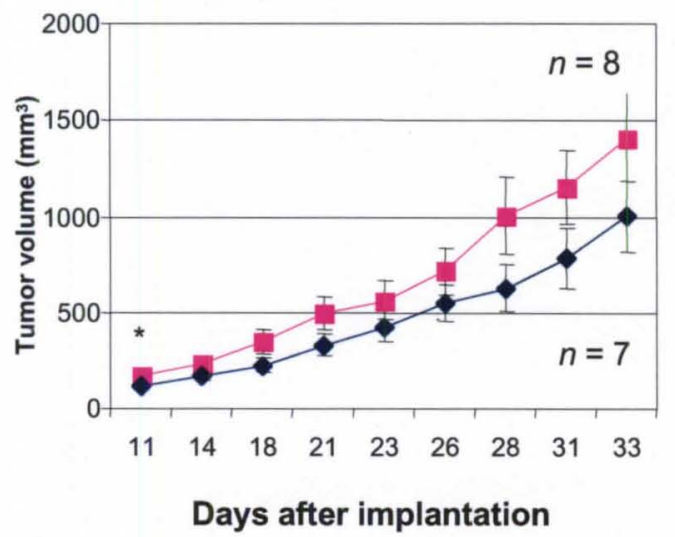

Figure 11: In vivo tumor growth curves of DU145 cells pretreated with 10 $\mu \mathrm{M}$ AS1411 for 72 hours. Number of cells and site of implantation as noted. 
pretreatment of prostate cancer cells with AS1411 inhibits tumor growth in vivo. With better in vivo injection technique, we feel confident that this difference will be statistically significant for most if not all time points.

\section{Long-term treatment with AS1411 in vivo showed no difference in tumor growth or outcome}

We attempted to mimic the delayed tumor regression seen in the AS1411 Phase I clinical trial by establishing DU145 subcutaneous tumors in nude mice and, once palpable, treating mice with daily intraperitoneal injections of AS1411 $(10 \mathrm{mg} / \mathrm{kg} / \mathrm{day})$ or negative vehicle for three weeks. We observed no significant difference in tumor volume between treated versus untreated mice (data not shown). We also analyzed survival in the two groups and again found no difference.

AS1411, as well as other aptamers, is known to be rapidly cleared from systemic circulation via the kidneys with a half-life on the order of minutes. Due to this pharmacokinetic feature, in clinical trials AS1411 was given by continuous infusion for seven days. The daily intraperitoneal injection model that we used for this experiment may thus not be an effective method to study aptamer pharmaceuticals. An experiment using an infusion method of drug delivery would likely be more appropriate, and we are planning to pursue this in the near future. Once a modified research protocol is approved, we will use implantable intraperitoneal pumps to deliver appropriate amounts of AS1411 over the course of 7 days.

\section{CONCLUSIONS}


Cancer research in recent years has seen the advent of numerous new therapies with efficacy in decreasing tumor volume, yet there have been relatively few breakthroughs leading to improvement in survival. This quandary may be due to CSCs. CSCs and TICs comprise an exciting new field of study experiencing exponential growth with far reaching implications in clinical oncology and cancer research. Tumor heterogeneity in general is an increasingly relevant concept. The field of oncology has moved historically from considering cancer to be a single disease, to believing every cancer within an organ to be the same, to realizing different cancer types exist within a given tissue type. Now the dawning realization is that the differences between cancer cells within a single malignancy must also be addressed. It is likely that in the near future there will be even more refined medical subdivisions in cancer histology and diagnosis that guide clinical treatment.

Given the growth in the study of TICs, a steady increase in the identification and development of TIC-targeting therapies is likely to follow. At present, only a handful of agents have been reported to deplete TICs. They include small molecule inhibitors of certain signaling pathways (e.g. Hedgehog, Akt, NF-кB, Notch), salinomycin, aurothiomalate, temozolamide, and a few oncolytic viruses. Our preliminary data suggest that AS1411 is effective in targeting and inhibiting TICs in prostate cancer cells. We have generated in vitro and in vivo data supporting our hypothesis. We have studied baseline levels of prostate TIC surface markers in DU145 prostate cancer cells and shown that AS1411 decreases them. Using the SP and ALDH assays we have shown that AS1411 also decreases functional markers. We have provided evidence that AS1411 decreases the clonogenicity of DU145 cells via demonstrating decreased colony formation in soft agar 
and decrease sphere survival in non-adherent culture. These markers and functional assays have all been described in the study of prostate TICs. Although the precise definitions of TIC versus CSC are still being shaped, cancer cells with these signatures have been shown repeatedly to be more tumorigenic, more invasive, and more drug resistant than other prostate cancer cells. Therefore, regardless if AS1411 only targets TICs rather than the true CSC compartment, or inhibits only a subtype of TICs, or, with changing definitions, does not target "TICs" at all, we are still providing evidence that AS1411 inhibits particularly clinically important and malignant subpopulations of prostate cancer cells.

The ex vivo pretreatment of cancer cells followed by subcutaneous implantation is a model that has recently been reported by a number of groups in the study of TICs. This model does not attempt to mimic the clinical scenario, but illustrates the effect of drugs on cancer cell growth under the complexities of mammalian physiology. Our data suggest that treatment with AS1411 slows the growth of prostate cancer cells in vivo. The longterm in vivo experiments that we performed (which are a truer model for clinical oncology) did not show a difference with AS1411 treatment in the growth of established subcutaneous prostate cancer tumors. As discussed, we feel the lack of effect is due to insufficiency with administration of the drug and intend to repeat this experiment with implantable drug delivery pumps to achieve sustained levels of the drug and to more accurately mimic the clinical trial protocols.

Although we have shown that AS1411 decreases signatures and markers associated with TICs, we realize that we are not able to conclude the fate of AS1411treated TICs from our data. As discussed in the introduction of this chapter, many 
possible fates exist for TICs including cell death, blocking of proliferation (cytostasis), and differentiation. To shed some light on the mechanism of downregulation of the $\mathrm{CD} 24^{\mathrm{lo}} / \mathrm{CD} 44^{\mathrm{hi}}$ stem cell signature with 72 hour treatment of AS1411 on monolayer DU145 cells, one planned experiment is to plate freshly sorted CD $24^{\mathrm{lo}} / \mathrm{CD} 44^{\mathrm{hi}}$ cells in monolayer cell culture conditions with or without $10 \mu \mathrm{M}$ AS1411, then harvest after 72 hours and stain for CD24, CD44, and with propidium iodide. The experiment will also be performed plating the sorted cells in non-adherent sphere culture conditions. Although sphere culture conditions less accurately mimic the conditions under which our assays were performed, at this time we are uncertain of general effects of monolayer culture conditions on the sorted cells. According to the literature, both serum and adhesion tend to induce differentiation of stem-like cells, therefore we will examine the cells under both monolayer and sphere culture conditions. We hope that investigations such as these using AS1411 on TICs will provide some insight on the fate of these cells.

One technical issue that needs to be addressed is appropriate controls. Most of these assays have never before been performed in our laboratory. Designing and optimizing these protocols required a great deal of time and resources, and thus in most cases we restricted our experiments to AS1411-treated and -untreated samples. Additional important controls to add to our study are classic, non-TIC targeting drugs and other oligomers at comparable doses. The question of appropriate doses of the classic chemotherapy doxorubicin has been discussed. An even more relevant chemotherapy control would be docetaxel, the first-line chemotherapy for CRPC (Petrioli, 2008) which is also used for other cancer types and has been reported not to target CSC/TIC subpopulations. In terms of oligomer controls, experts in the field opine a lack of true 
relevant control molecules. It is not possible to use a scrambled version of AS1411 as this ODN is comprised solely of guanines and thymines, and scrambling the order of these bases does not provide sufficient variation from the original sequence. The standard oligomer control that our group has used in many other publications to contrast with AS1411 is "CRO," a C-rich 26-base oligomer which replaces the guanines of AS1411 with cytosines. Although CRO is limited in relevance because it is not a G-quaduplex, we use this molecule because it is an ODN of the same length as AS1411 that is known to have no biological activity in any assay our laboratory has performed. Plans are underway to repeat key experiments with CRO.

The field of CSC/TICs, though exciting and rapidly expanding, is still only on the order of a decade old. Even younger is the field of solid tumor CSCs; the establishment of benign solid organ stem cells was many decades behind hematopoietic stem cells, and solid tumor CSCs have naturally lagged behind their leukemic counterparts as well. As in any new field of research, there is a high degree of controversy over virtually every aspect of CSCs, even their very existence. The validity of methods used to study stemlike cancer cell populations, including the SP and ALDH assays that we describe here, have been proposed, questioned, and refuted in turn. We have attempted to select signatures and assays for which the greatest consensus has been built, but it will take time before the methodology in the study of TICs is universally accepted. Even more fundamentally, questions such as whether or not all tumors have CSCs and if neutralizing or destroying CSCs actually translates into clinical benefit have yet to be answered.

In this project, we performed proof-of-principle studies to examine the effect of a known anticancer aptamer on TIC subpopulations. We do not attempt to prove the CSC 
hypothesis, to answer fundamental questions in the field, or to validate the signatures that have been reported to identify prostate TICs. Although we have been as rigorous as possible in our study of TICs, we acknowledge that we are necessarily utilizing unproven assays. To compensate for this issue, we tested our hypothesis that AS1411 is a TICtargeting drug by several different methods, each of which in actuality is likely to assess a slightly different yet overlapping cancer cell subset. While not conclusive, taken together our data support our hypothesis and provide a strong argument that AS1411 affects TICs. This does not preclude effects on non-TIC cells, and indeed previous data from our group indicate a strong anticancer effect on bulk tumor cells for a number of different cancer types.

In the future, we would like examine the effect of AS1411 on the gene expression of spherocytes to see if these data provide any insight to the mechanism of action of AS1411 on TICs. We have plans to perform a qPCR stem cell gene array with cDNA created from spherocytes that have been treated with AS1411 versus negative vehicle. We plan to study the mechanism of action of this inhibition on TICs to see if it differs from that of the bulk tumor cells, to repeat and refine our assays to achieve statistical significance using appropriate controls, and to prepare a manuscript for publication. Ideally we would also like to repeat key experiments using clinical specimens. Our work will be among the very first articles to combine the fields of aptamers and TICs, and the first to present data that an anticancer aptamer inhibits the TIC class of cancer cells. 


\section{CHAPTER 6}

\section{FINAL REMARKS}

\section{Summary of major findings and their significance}

\section{G-rich oligodeoxynucleotide libraries}

The properties of a molecular solution of any kind that contains a mixture of species are, most logically, a summative function of the individual molecules that comprise it. Thus, the properties of the solution may be argued to be representative of the predominance of molecules. We found that GT and GC ODN 26mer libraries, each containing millions of different G-rich sequences, exhibit selective anticancer activity as a whole. This implies that rather than being a rare occurrence, this feature is a common property for the different individual sequences within these particular libraries. Ours is the first report of a large, complex library of ODNs, rather than a single sequence or a few known sequences in combination, exhibiting cancer-selective antiproliferative activity (Chapter 2, Appendix A).

We found that the selective antiproliferative activity against cancer cells was only seen in G-rich libraries (though not all G-rich libraries) and generally corresponded with formation of G-quadruplexes, molecular stability against nucleases, and increased cellular uptake. The causative relationship between the different biophysical and biological properties we assayed was not determined, although we suggest that protein binding may be required for molecular stability. Along this line of reasoning, a follow up 
study could verify that the band "Bl" identified most strongly in the GT libraries is indeed nucleolin as we suspect, and that the protein is the same across the different ODN libraries (i.e. that $\mathrm{B} 1$ contains the same protein in all samples). Although nucleolin is a required factor for AS1411-mediated anticancer activity, these results suggest that factors other than nucleolin binding play a role in determining activity; future research could help us understand why some libraries exhibited this band but did not have antiproliferative activity against cancer cells. One method to assess the relative binding affinities is the competitive EMSA using radiolabeled TEL (telomerase sequence) as a competitor for nucleolin binding. Caveats include the possibility that different ODN species may bind to different sites or to different forms of nucleolin. As TEL is a known G-quadruplex-forming sequence, it is logical that libraries with high levels of Gquadruplex formation would compete with TEL, but others would not. This could provide information about the relative importance of G-quadruplex formation versus G-richness to anticancer activity.

\section{GC library and poly-dC binding targets}

Both GC and C-rich nucleic acid sequences have important roles in cellular biology (discussed fully in Chapter 3). Arising from the protein binding assay of the ODN libraries chapter, we identified a handful of proteins that are bound by the GC 26 mer library and the poly-dC oligomer. Some of these have been previously reported while some were newly identified by us. A number of the identified proteins have known effects in cancer biology in general and prostate cancer in particular. As discussed in the chapter, important follow-up experiments include foremost the verification of the protein targets 
and a study of their biological relevance. Many other follow up projects, particularly involving the GC library, could stem from these data. The mechanism of the GC library's cancer-selective antiproliferative activity is likely different from the GT-library; this is a novel finding and could be an important development. Like the GT libraries, the GC library had potent antiproliferative activity against lung and prostate cancer cells and little effect on benign fibroblasts. In contrast, the GC library had markedly attenuated activity against the MCF7 breast cancer cells, while these cells were the most vulnerable cancer type to the GT libraries. Discovery of the basis of this difference in antiproliferative activity could be a complete research project in and of itself with implications not only in oncology but also basic cellular biology. Further studies with the GC library may lead to new strategies in the advancement of cancer-selective therapies.

\section{Identification of a new anti-prostate cancer aptamer via SELEX}

Despite technical hurdles, we identified a candidate aptamer evolved via SELEX from the GT library that has enhanced cancer-selective antiproliferative activity and increased nuclease resistance relative to its parent library (Chapter 4). Although our SELEX protocol was designed to select for sequences with efficient cellular uptake, we were unable to exhibit an increase in uptake by the GTcap sequence despite employing a variety of methods. ODN uptake is a challenging phenomenon to study, and at this time the mechanism of the cellular uptake of G-quadruplex ODNs is poorly understood by the scientific community as a whole. One technical difficulty that we encountered is intrinsic variation of fluorescence of ODN samples. Some of this variation may be due to difference in manufacturing conditions, but this issue is particularly associated with 
quenching of the fluorophore by G-quadruplexes. Fluorescent ODNs can only be used in comparison if measured fluorescence is proportional to ODN concentration across samples. We found inconsistent fluorescence among our fluorophore-labeled ODN stocks. Though we attempted to compensate for this difference both mathematically and experimentally, this incongruity makes any experiment comparing these ODNs questionable.

More recently, a new paradigm for AS1411 cellular uptake has developed from our laboratory (92). In short, evidence suggests that it is not the degree of cellular uptake that is the key to the cancer-selective antiproliferative activity of AS1411, but the method of cellular uptake. The major mechanism of uptake for cancer cells is macropinocytosis while the predominant mechanism for benign cells is non-macropinocytic endocytosis. AS1411 triggers hyperstimulation of macropinocytosis only in the cancer cells, and this hyperstimulation appears to require nucleolin though the mechanism of this interaction is incompletely understood at this time. In accordance with the literature, we saw increased cellular uptake with our "active" G-rich samples in our study of ODN libraries, and the degree of uptake appeared to correlate with antiproliferative activity (Chapter 2). Although we were unable to demonstrate increased cellular uptake with the GTcap sequence, enhanced cellular uptake is a known general characteristic of GROs. Higher uptake is a valuable pharmacokinetic property that may have many clinical and experimental applications, but the new data regarding macropinocytosis and AS1411 suggests that the paradigm of higher intracellular uptake corresponding with higher efficacy is overly simplistic. We now believe that both the specific mechanism of uptake as well as high intracellular concentration play important roles in the selective anticancer 
activity of AS1411 and perhaps GROs in general.

Of note, our newly evolved aptamer (GTcap) is G-rich and does appear to form a G-quadruplex, have enhanced resistance to nuclease degradation relative to its parent library, and exhibit greater complexity of protein binding. This finding is in agreement with our previous results using ODN libraries, namely that selective antiproliferative activity against cancer cells may correspond with and require all these features. Therefore, it is possible that we have inadvertently selected the GTcap sequence based on its superior nuclease-resistance, rather than increased cellular uptake.

\section{AS1411 inhibits prostate cancer tumor initiating cells}

We studied the effect of AS1411, a known G-rich, G-quadruplex-forming aptamer, on subpopulations of prostate cancer cells reported to have enhanced tumorigenicity (i.e. TICs; Chapter 5). By all assays tested, AS1411 decreased prostate TIC signatures. Most excitingly, prostaspheres treated with AS1411 had shorter survival than both negative vehicle and the classic chemotherapy doxorubicin. This trend also translated to the in vivo realm; preliminary studies showed that cancer cells pretreated with AS1411 exhibited slowed tumor growth compared to control treated samples.

We acknowledge the limitations of the field, for CSC/TICs in general and solid tumor and prostate TICs in particular, but believe that our data collectively support the hypothesis that one of the ways by which AS1411 acts against cancer is by inhibiting TICs. We are interested to study whether or not this effect is exclusive to AS1411, or rather a general property of other GROs as well. Experiments to test the effect of other Grich samples including ODN libraries (GA, GC, and GT 26mers) and well as our selected 
GTcap aptamer on prostasphere survival are underway. We are also testing some non-Grich libraries and ODNs in parallel. We know that some of these samples form Gquadruplexes and some do not; we may thus be able to correlate G-richness versus Gquadruplex formation with inhibition of prostasphere survival.

\section{Overall conclusions and future directions}

Our hypothesis is that guanine-rich oligonucleotides are optimal for the development of new aptamers for use in the treatment of cancer. We have performed experiments using GRO libraries correlating G-quadruplex formation with cancerselective antiproliferative activity. Following from this study we undertook and identified unique GC library-binding proteins. We performed SELEX and managed to evolve a Grich aptamer with more potent anticancer activity and greater nuclease resistance than its G-rich parent library. And lastly, we generated evidence that a first in class G-rich anticancer aptamer inhibits TICs. Taken as a whole, this body of work supports the hypothesis that GROs have great potential in the development of cancer therapies.

For context, we have discussed specific possible future studies for each subproject (ODN libraries, protein target identification, SELEX, and TIC) in depth within each chapter. Clearly much more work could be done in every aspect of this project. Our immediate plans are to strengthen our data involving AS1411 and prostate TICs to prepare a manuscript for submission. We also plan to study the effect of other G-rich and non-G-rich libraries and ODNs via the sphere survival assay. The results may have implications on the anticancer activity of the libraries, and if sphere survival correlates with the previous anticancer activity seen, further study will be warranted. 
As previously discussed, according to the literature, GROs have distinct properties, namely nuclease resistance and enhanced cellular uptake that appear to be related to G-quadruplex formation. These properties may be accountable for the cancerselective antiproliferative activity seen with AS1411, GRO libraries, and the GTcap sequence, and could perhaps be a common feature of sequences that form Gquadruplexes. The causative relationship between these GRO-associated properties has not been fully determined. It is widely held that G-quadruplexes are resistant to nucleases due to the intrinsic stability of their secondary structure, and that this stability affords Gquadruplexes the opportunity to reach a protein target (e.g. nucleolin) and bind it. Our data suggest that it is in fact protein binding that confers molecular stability rather than vice versa (Chapter 2, Appendix A). We propose a new model by which GROs are more likely to bind to a protein such as nucleolin which provides stability against serum and intracellular nucleases. This protein binding may or may not depend on G-quaduplex formation. Regardless, with this stability, GROs survive and are able to be taken up by the cell and reach other targets.

Although the determinants of the mechanism of cellular uptake of G-quadruplex ODNs are unclear, it is known that GROs may be taken up by a variety of ways and have a number of effects, including cell death, cytostasis, and antiviral activity ( $\underline{18})$. In accordance with Dr. Reyes-Reyes' recent work, cancer cells take up G-quadruplexes by macropinocytosis, a process which may then become dysregulated by a nucleolindependent mechanism and lead to a novel kind of cell death. Clearly there is more information to be discovered, for example why the GC 26 mer library was only active against certain cancer cell types while GT 26mer libraries were active against all cancer 
types tested, and whether cancer-selective hyperstimulation of macropinocytosis is specific to AS1411 or a general property of GROs.

\section{The future of anticancer aptamers}

Aptamers as pharmaceuticals, especially in the context of oncology, are a worthy field of study. Aptamers have many features that make them desirable for drug development and commercial manufacture. The cost of drug development is notoriously high, and of the total potential pharmaceuticals that enter the research and development process, less than $1 \%$ will ever reach market. Aptamers are inexpensive and easy to manufacture which is important not only in the context of providing drug to patients, but also in the scientific study of these molecules. Aptamers typically have low side effects as evidenced by the lack of dose-limiting toxicities in the clinical trials of AS1411. Features that makes GROs good candidates against cancer in general also makes them good candidates for treatments against CSCs, and there are additional features (previously discussed in Chapter 1) that may make them particularly well-suited to anti-CSC therapy.

To date, there is a single report of an RNA anti-EpCAM aptamer which may prove to inhibit CSCs. However, no one has yet reported using SELEX to target whole CSC/TICs. In conclusion, we suggest that in the future it will become technically possible to employ SELEX using a G-rich library to deliberately develop aptamers that target CSC/TICs. As the field of CSCs and TICs matures, the subpopulations of cancer cells that are enriched in tumorigenic capabilities will become better characterized. It will then be possible to use these clinically important subpopulations as targets of whole cell SELEX using a G-rich starting library as we have described here. TICs may be sorted out 
from clinical tumor samples based on functional or surface markers and if necessary established as primary cultures at the time of surgical resection. Cancer patients typically require a post-surgical recovery period before beginning a chemotherapy regimen. During this time, automated SELEX could be performed upon each individual patient's cancer cells. Although automation for cell-SELEX is undoubtedly further away than for SELEX using a molecular target, we believe cell-SELEX would be preferable for tailored therapy as the SELEX could be performed without knowledge of a target on the molecular level which may or may not be relevant for that particular patient. As aptamers are remarkably safe and non-toxic pharmaceuticals, the issue of dangerous side effects is less concerning. At this time, tailored oncology therapies are limited to determining which patients will benefit most from the available chemotherapies (e.g. giving anti-estrogen medication only to those breast cancer patients whose cancers are $\mathrm{ER}^{+}$). We describe a scenario of the future, which will involve custom made cancer therapies for each individual and their unique cancer.

The overall aim of our project is to identify aptamers for use as oncology therapeutics (i.e. chemotherapy, drug-delivery vehicle, imaging) and to study the mechanisms of the unusual and potentially useful properties of GROs. A better understanding of how GROs work may lead to the development of specific and selective anticancer drugs, both vehicles (for targeted delivery of small molecules or siRNAs) and frank aptamer pharmaceuticals. In this body of work, we have generated and discussed a diverse collection of data related to GROs and their effects on cancer cells. Our work has contributed to the fields of G-rich oligonucleotides, aptamers, cancer therapeutics, and tumor initiating cells in overlapping and sometimes wholly novel ways. A number of our 
findings have previously been unreported, and comprise a small yet significant contribution to the literature. 


\section{REFERENCES}

1. Kochanek, K.X., J; Murphy, SL; Minino, AM; Kung, HC. (2011), National Vital Statistics Reports, Vol. 59, pp. 1-69.

2. American Cancer Society. (2010) Cancer Facts \& Figures 2010.

3. Lipscomb, J. (2008) Estimating the cost of cancer care in the United States: a work very much in progress. J Natl Cancer Inst, 100, 607-610.

4. Petrylak, D.P., Tangen, C.M., Hussain, M.H., Lara, P.N., Jr., Jones, J.A., Taplin, M.E., Burch, P.A., Berry, D., Moinpour, C., Kohli, M. et al. (2004) Docetaxel and estramustine compared with mitoxantrone and prednisone for advanced refractory prostate cancer. $N$ Engl $J$ Med, 351, 1513-1520.

5. Tannock, I.F., de Wit, R., Berry, W.R., Horti, J., Pluzanska, A., Chi, K.N., Oudard, S., Theodore, C., James, N.D., Turesson, I. et al. (2004) Docetaxel plus prednisone or mitoxantrone plus prednisone for advanced prostate cancer. $N E n g l$ $J$ Med, 351, 1502-1512.

6. Beltran, H., Beer, T.M., Carducci, M.A., de Bono, J., Gleave, M., Hussain, M., Kelly, W.K., Saad, F., Sternberg, C., Tagawa, S.T. et al. (2011) New therapies for castration-resistant prostate cancer: efficacy and safety. Eur Urol, 60, 279-290.

7. Takasugi, M., Guendouz, A., Chassignol, M., Decout, J.L., Lhomme, J., Thuong, N.T. and Helene, C. (1991) Sequence-specific photo-induced cross-linking of the 
two strands of double-helical DNA by a psoralen covalently linked to a triple helix-forming oligonucleotide. Proc Natl Acad Sci U S A, 88, 5602-5606.

8. Helene, C., Thuong, N.T. and Harel-Bellan, A. (1992) Control of gene expression by triple helix-forming oligonucleotides. The antigene strategy. Ann NY Acad Sci, $660,27-36$.

9. Duca, M., Vekhoff, P., Oussedik, K., Halby, L. and Arimondo, P.B. (2008) The triple helix: 50 years later, the outcome. Nucleic Acids Res, 36, 5123-5138.

10. Green, P.J., Pines, O. and Inouye, M. (1986) The role of antisense RNA in gene regulation. Annu Rev Biochem, 55, 569-597.

11. Gleave, M.E. and Monia, B.P. (2005) Antisense therapy for cancer. Nat Rev Cancer, 5, 468-479.

12. Pecot, C.V., Calin, G.A., Coleman, R.L., Lopez-Berestein, G. and Sood, A.K. (2011) RNA interference in the clinic: challenges and future directions. Nat Rev Cancer, 11, 59-67.

13. Tomita, N., Ogihara, T. and Morishita, R. (2003) Transcription factors as molecular targets: molecular mechanisms of decoy ODN and their design. Curr Drug Targets, 4, 603-608.

14. Holtick, U., Scheulen, M.E., von Bergwelt-Baildon, M.S. and Weihrauch, M.R. (2011) Toll-like receptor 9 agonists as cancer therapeutics. Expert Opin Investig Drugs, 20, 361-372.

15. Lubaroff, D.M. and Karan, D. (2009) CpG oligonucleotide as an adjuvant for the treatment of prostate cancer. Adv Drug Deliv Rev, 61, 268-274.

16. Ellington, A.D. and Szostak, J.W. (1990) In vitro selection of RNA molecules that 
bind specific ligands. Nature, 346, 818-822.

17. Ireson, C.R. and Kelland, L.R. (2006) Discovery and development of anticancer aptamers. Mol Cancer Ther, 5, 2957-2962.

18. Bates, P.J., Laber, D.A., Miller, D.M., Thomas, S.D. and Trent, J.O. (2009) Discovery and development of the G-rich oligonucleotide AS1411 as a novel treatment for cancer. Exp Mol Pathol, 86, 151-164.

19. Syed, M.A. and Pervaiz, S. (2010) Advances in aptamers. Oligonucleotides, 20, 215-224.

20. Bunka, D.H., Platonova, O. and Stockley, P.G. (2010) Development of aptamer therapeutics. Curr Opin Pharmacol, 10, 557-562.

21. Zimmermann, T.S., Lee, A.C., Akinc, A., Bramlage, B., Bumcrot, D., Fedoruk, M.N., Harborth, J., Heyes, J.A., Jeffs, L.B., John, M. et al. (2006) RNAi-mediated gene silencing in non-human primates. Nature, 441, 111-114.

22. Peer, D., Park, E.J., Morishita, Y., Carman, C.V. and Shimaoka, M. (2008) Systemic leukocyte-directed siRNA delivery revealing cyclin D1 as an antiinflammatory target. Science, 319, 627-630.

23. Sliva, K. and Schnierle, B.S. (2010) Selective gene silencing by viral delivery of short hairpin RNA. Virol $J, 7,248$.

24. Bartlett, D.W. and Davis, M.E. (2007) Physicochemical and biological characterization of targeted, nucleic acid-containing nanoparticles. Bioconjug Chem, 18, 456-468.

25. Kaneda, Y. (2010) Update on non-viral delivery methods for cancer therapy: possibilities of a drug delivery system with anticancer activities beyond delivery 
as a new therapeutic tool. Expert Opin Drug Deliv, 7, 1079-1093.

26. Shim, M.S. and Kwon, Y.J. (2010) Efficient and targeted delivery of siRNA in vivo. Febs $J, 277,4814-4827$.

27. Singha, K., Namgung, R. and Kim, W.J. (2011) Polymers in Small-Interfering RNA Delivery. Oligonucleotides.

28. Lu, P.Y., Xie, F.Y. and Woodle, M.C. (2005) Modulation of angiogenesis with siRNA inhibitors for novel therapeutics. Trends Mol Med, 11, 104-113.

29. Han, H., Cliff, C.L. and Hurley, L.H. (1999) Accelerated assembly of Gquadruplex structures by a small molecule. Biochemistry, 38, 6981-6986.

30. Bates, P.J., Choi, E.W. and Nayak, L.V. (2009) G-rich oligonucleotides for cancer treatment. Methods Mol Biol, 542, 379-392.

31. Maloney, D.G., Grillo-Lopez, A.J., White, C.A., Bodkin, D., Schilder, R.J., Neidhart, J.A., Janakiraman, N., Foon, K.A., Liles, T.M., Dallaire, B.K. et al. (1997) IDEC-C2B8 (Rituximab) anti-CD20 monoclonal antibody therapy in patients with relapsed low-grade non-Hodgkin's lymphoma. Blood, 90, 21882195.

32. Gragoudas, E.S., Adamis, A.P., Cunningham, E.T., Jr., Feinsod, M. and Guyer, D.R. (2004) Pegaptanib for neovascular age-related macular degeneration. $N$ Engl $J$ Med, 351, 2805-2816.

33. Bates, P.J., Kahlon, J.B., Thomas, S.D., Trent, J.O. and Miller, D.M. (1999) Antiproliferative activity of G-rich oligonucleotides correlates with protein binding. J Biol Chem, 274, 26369-26377.

34. Xu, X., Hamhouyia, F., Thomas, S.D., Burke, T.J., Girvan, A.C., McGregor, W.G., 
Trent, J.O., Miller, D.M. and Bates, P.J. (2001) Inhibition of DNA replication and induction of S phase cell cycle arrest by G-rich oligonucleotides. $J$ Biol Chem, 276, 43221-43230.

35. Dapic, V., Bates, P.J., Trent, J.O., Rodger, A., Thomas, S.D. and Miller, D.M. (2002) Antiproliferative activity of G-quartet-forming oligonucleotides with backbone and sugar modifications. Biochemistry, 41, 3676-3685.

36. Dapic, V., Abdomerovic, V., Marrington, R., Peberdy, J., Rodger, A., Trent, J.O. and Bates, P.J. (2003) Biophysical and biological properties of quadruplex oligodeoxyribonucleotides. Nucleic Acids Res, 31, 2097-2107.

37. Girvan, A.C., Teng, Y., Casson, L.K., Thomas, S.D., Juliger, S., Ball, M.W., Klein, J.B., Pierce, W.M., Jr., Barve, S.S. and Bates, P.J. (2006) AGRO100 inhibits activation of nuclear factor-kappaB (NF-kappaB) by forming a complex with NFkappaB essential modulator (NEMO) and nucleolin. Mol Cancer Ther, 5, 17901799.

38. Mongelard, F. and Bouvet, P. (2010) AS-1411, a guanosine-rich oligonucleotide aptamer targeting nucleolin for the potential treatment of cancer, including acute myeloid leukemia. Curr Opin Mol Ther, 12, 107-114.

39. Leeb, C., Jurga, M., McGuckin, C., Forraz, N., Thallinger, C., Moriggl, R. and Kenner, L. (2011) New perspectives in stem cell research: beyond embryonic stem cells. Cell Prolif, 44 Suppl 1, 9-14.

40. Pelacho, B., Mazo, M., Gavira, J.J. and Prosper, F. (2011) Adult stem cells: from new cell sources to changes in methodology. J Cardiovasc Transl Res, 4, 154-160.

41. Snippert, H.J., Haegebarth, A., Kasper, M., Jaks, V., van Es, J.H., Barker, N., van 
de Wetering, M., van den Born, M., Begthel, H., Vries, R.G. et al. (2010) Lgr6 marks stem cells in the hair follicle that generate all cell lineages of the skin. Science, 327, 1385-1389.

42. Tsujimura, A., Fujita, K., Komori, K., Takao, T., Miyagawa, Y., Takada, S., Matsumiya, K., Nonomur, N. and Okuyama, A. (2007) Prostatic stem cell marker identified by cDNA microarray in mouse. J Urol, 178, 686-691.

43. Bomken, S., Fiser, K., Heidenreich, O. and Vormoor, J. (2010) Understanding the cancer stem cell. Br J Cancer, 103, 439-445.

44. Chen, S.Y., Huang, Y.C., Liu, S.P., Tsai, F.J., Shyu, W.C. and Lin, S.Z. (2011) An overview of concepts for cancer stem cells. Cell Transplant, 20, 113-120.

45. Tysnes, B.B. (2010) Tumor-initiating and -propagating cells: cells that we would like to identify and control. Neoplasia, 12, 506-515.

46. O'Brien, C.A., Kreso, A. and Jamieson, C.H. (2010) Cancer stem cells and selfrenewal. Clin Cancer Res, 16, 3113-3120.

47. Bonnet, D. and Dick, J.E. (1997) Human acute myeloid leukemia is organized as a hierarchy that originates from a primitive hematopoietic cell. Nat Med, 3, 730737.

48. Lapidot, T., Sirard, C., Vormoor, J., Murdoch, B., Hoang, T., Caceres-Cortes, J., Minden, M., Paterson, B., Caligiuri, M.A. and Dick, J.E. (1994) A cell initiating human acute myeloid leukaemia after transplantation into SCID mice. Nature, $367,645-648$.

49. Reya, T., Morrison, S.J., Clarke, M.F. and Weissman, I.L. (2001) Stem cells, cancer, and cancer stem cells. Nature, 414, 105-111. 
50. Clarke, M.F., Dick, J.E., Dirks, P.B., Eaves, C.J., Jamieson, C.H., Jones, D.L., Visvader, J., Weissman, I.L. and Wahl, G.M. (2006) Cancer stem cells-perspectives on current status and future directions: AACR Workshop on cancer stem cells. Cancer Res, 66, 9339-9344.

51. Graham, S.M., Jorgensen, H.G., Allan, E., Pearson, C., Alcorn, M.J., Richmond, L. and Holyoake, T.L. (2002) Primitive, quiescent, Philadelphia-positive stem cells from patients with chronic myeloid leukemia are insensitive to STI571 in vitro. Blood, 99, 319-325.

52. Liu, F.S. (2009) Mechanisms of chemotherapeutic drug resistance in cancer therapy--a quick review. Taiwan J Obstet Gynecol, 48, 239-244.

53. Elrick, L.J., Jorgensen, H.G., Mountford, J.C. and Holyoake, T.L. (2005) Punish the parent not the progeny. Blood, 105, 1862-1866.

54. Klein, I., Sarkadi, B. and Varadi, A. (1999) An inventory of the human ABC proteins. Biochim Biophys Acta, 1461, 237-262.

55. Lou, H. and Dean, M. (2007) Targeted therapy for cancer stem cells: the patched pathway and $\mathrm{ABC}$ transporters. Oncogene, 26, 1357-1360.

56. Borovski, T., De Sousa, E.M.F., Vermeulen, L. and Medema, J.P. (2011) Cancer stem cell niche: the place to be. Cancer Res, 71, 634-639.

57. Hicke, B.J., Stephens, A.W., Gould, T., Chang, Y.F., Lynott, C.K., Heil, J., Borkowski, S., Hilger, C.S., Cook, G., Warren, S. et al. (2006) Tumor targeting by an aptamer. $J$ Nucl Med, 47, 668-678.

58. Perkins, A.C. and Missailidis, S. (2007) Radiolabelled aptamers for tumour imaging and therapy. $Q J \mathrm{Nucl} \mathrm{Med} \mathrm{Mol} \mathrm{Imaging,} \mathrm{51,} \mathrm{292-296.}$ 
59. Choi, E.W., Nayak, L.V. and Bates, P.J. (2010) Cancer-selective antiproliferative activity is a general property of some G-rich oligodeoxynucleotides. Nucleic Acids Res, 38, 1623-1635.

60. Mashima, T., Matsugami, A., Nishikawa, F., Nishikawa, S. and Katahira, M. (2009) Unique quadruplex structure and interaction of an RNA aptamer against bovine prion protein. Nucleic Acids Res, 37, 6249-6258.

61. Matsugami, A., Mashima, T., Nishikawa, F., Murakami, K., Nishikawa, S., Noda, K., Yokoyama, T. and Katahira, M. (2008) Structural analysis of r(GGA)4 found in RNA aptamer for bovine prion protein. Nucleic Acids Symp Ser (Oxf), 179-180.

62. Portela, A. and Esteller, M. (2010) Epigenetic modifications and human disease. Nat Biotechnol, 28, 1057-1068.

63. Rodriguez-Paredes, M. and Esteller, M. (2011) Cancer epigenetics reaches mainstream oncology. Nat Med, 17, 330-339.

64. Woodson, K., O'Reilly, K.J., Ward, D.E., Walter, J., Hanson, J., Walk, E.L. and Tangrea, J.A. (2006) CD44 and PTGS2 methylation are independent prognostic markers for biochemical recurrence among prostate cancer patients with clinically localized disease. Epigenetics, 1, 183-186.

65. Lavrov, S.A. and Kibanov, M.V. (2007) Noncoding RNAs and chromatin structure. Biochemistry (Mosc), 72, 1422-1438.

66. Hammann, C. and Westhof, E. (2007) Searching genomes for ribozymes and riboswitches. Genome Biol, 8, 210.

67. Todd, A.K., Johnston, M. and Neidle, S. (2005) Highly prevalent putative quadruplex sequence motifs in human DNA. Nucleic Acids Res, 33, 2901-2907. 
68. Gilbert, D.E. and Feigon, J. (1999) Multistranded DNA structures. Curr Opin Struct Biol, 9, 305-314.

69. Sun, D. and Hurley, L.H. (2009) The importance of negative superhelicity in inducing the formation of G-quadruplex and i-motif structures in the c-Myc promoter: implications for drug targeting and control of gene expression. $J$ Med Chem, 52, 2863-2874.

70. Gueron, M. and Leroy, J.L. (2000) The i-motif in nucleic acids. Curr Opin Struct Biol, 10, 326-331.

71. Phan, A.T., Gueron, M. and Leroy, J.L. (2000) The solution structure and internal motions of a fragment of the cytidine-rich strand of the human telomere. $J \mathrm{Mol}$ Biol, 299, 123-144.

72. Sen, D. and Gilbert, W. (1992) Novel DNA superstructures formed by telomerelike oligomers. Biochemistry, 31, 65-70.

73. Meyer, M.M., Ames, T.D., Smith, D.P., Weinberg, Z., Schwalbach, M.S., Giovannoni, S.J. and Breaker, R.R. (2009) Identification of candidate structured RNAs in the marine organism 'Candidatus Pelagibacter ubique'. BMC Genomics, $10,268$.

74. Poiata, E., Meyer, M.M., Ames, T.D. and Breaker, R.R. (2009) A variant riboswitch aptamer class for S-adenosylmethionine common in marine bacteria. Rna, 15, 2046-2056.

75. Cloke, B., Shah, K., Kaneda, H., Lavery, S., Trew, G., Fusi, L., Higham, J., Dina, R.E., Ghaem-Maghami, S., Ellis, P. et al. (2010) The poly(c)-binding protein-1 regulates expression of the androgen receptor. Endocrinology, 151, 3954-3964. 
76. Yeap, B.B., Voon, D.C., Vivian, J.P., McCulloch, R.K., Thomson, A.M., Giles, K.M., Czyzyk-Krzeska, M.F., Furneaux, H., Wilce, M.C., Wilce, J.A. et al. (2002) Novel binding of $\mathrm{HuR}$ and poly(C)-binding protein to a conserved UC-rich motif within the 3'-untranslated region of the androgen receptor messenger RNA. J Biol Chem, 277, 27183-27192.

77. Gehring, K., Leroy, J.L. and Gueron, M. (1993) A tetrameric DNA structure with protonated cytosine.cytosine base pairs. Nature, 363, 561-565.

78. Snoussi, K., Bulte, J.W., Gueron, M. and van Zijl, P.C. (2003) Sensitive CEST agents based on nucleic acid imino proton exchange: detection of poly(rU) and of a dendrimer-poly(rU) model for nucleic acid delivery and pharmacology. Magn Reson Med, 49, 998-1005.

79. Zhou, J., Wei, C., Jia, G., Wang, X., Feng, Z. and Li, C. (2010) Formation of imotif structure at neutral and slightly alkaline pH. Mol Biosyst, 6, 580-586.

80. Peng, Y., Li, X., Ren, J. and Qu, X. (2007) Single-walled carbon nanotubes binding to human telomeric i-motif DNA: significant acceleration of S1 nuclease cleavage rate. Chem Commun (Camb), 5176-5178.

81. Mimori, T., Akizuki, M., Yamagata, H., Inada, S., Yoshida, S. and Homma, M. (1981) Characterization of a high molecular weight acidic nuclear protein recognized by autoantibodies in sera from patients with polymyositis-scleroderma overlap. J Clin Invest, 68, 611-620.

82. Muller, C., Paupert, J., Monferran, S. and Salles, B. (2005) The double life of the $\mathrm{Ku}$ protein: facing the DNA breaks and the extracellular environment. Cell Cycle, 4, 438-441. 
83. Gullo, C., Au, M., Feng, G. and Teoh, G. (2006) The biology of Ku and its potential oncogenic role in cancer. Biochim Biophys Acta, 1765, 223-234.

84. Kwok, S.C., Liu, X. and Daskal, I. (2001) Molecular cloning, expression, localization, and gene organization of PTX1, a human nuclear protein that is downregulated in prostate cancer. DNA Cell Biol, 20, 349-357.

85. Zhou, W., Li, H., Feng, X.L., Wang, L., Zhu, B., Yao, K.T. and Ren, C.P. (2007) [PTX1 in nasopharyngeal carcinoma by RNAi technology]. Zhong Nan Da Xue Xue Bao Yi Xue Ban, 32, 235-240.

86. Liu, X., Daskal, I. and Kwok, S.C. (2003) Effects of PTX1 expression on growth and tumorigenicity of the prostate cancer cell line PC-3. DNA Cell Biol, 22, 469474.

87. Yang, Y.F., Chou, M.Y., Fan, C.Y., Chen, S.F., Lyu, P.C., Liu, C.C. and Tseng, T.L. (2008) The possible interaction of CDA14 and protein elongation factor lalpha. Biochim Biophys Acta, 1784, 312-318.

88. Gonzalez, V., Guo, K., Hurley, L. and Sun, D. (2009) Identification and characterization of nucleolin as a c-myc G-quadruplex-binding protein. $J$ Biol Chem, 284, 23622-23635.

89. Sun, D., Guo, K. and Shin, Y.J. (2011) Evidence of the formation of G-quadruplex structures in the promoter region of the human vascular endothelial growth factor gene. Nucleic Acids Res, 39, 1256-1265.

90. Mao, Y., Mehl, I.R. and Muller, M.T. (2002) Subnuclear distribution of topoisomerase I is linked to ongoing transcription and p53 status. Proc Natl Acad Sci US A, 99, 1235-1240. 
91. Teng, Y., Girvan, A.C., Casson, L.K., Pierce, W.M., Jr., Qian, M., Thomas, S.D. and Bates, P.J. (2007) AS1411 alters the localization of a complex containing protein arginine methyltransferase 5 and nucleolin. Cancer Res, 67, 10491-10500.

92. Reyes-Reyes, E.M., Teng, Y. and Bates, P.J. (2010) A new paradigm for aptamer therapeutic AS1411 action: uptake by macropinocytosis and its stimulation by a nucleolin-dependent mechanism. Cancer Res, 70, 8617-8629.

93. Klimek-Tomczak, K., Wyrwicz, L.S., Jain, S., Bomsztyk, K. and Ostrowski, J. (2004) Characterization of hnRNP K protein-RNA interactions. $J \mathrm{Mol} \mathrm{Biol}, \mathbf{3 4 2}$, 1131-1141.

94. Choi, H.S., Hwang, C.K., Song, K.Y., Law, P.Y., Wei, L.N. and Loh, H.H. (2009) Poly(C)-binding proteins as transcriptional regulators of gene expression. Biochem Biophys Res Commun, 380, 431-436.

95. Makeyev, A.V. and Liebhaber, S.A. (2002) The poly(C)-binding proteins: a multiplicity of functions and a search for mechanisms. Rna, 8, 265-278.

96. Pino, I., Pio, R., Toledo, G., Zabalegui, N., Vicent, S., Rey, N., Lozano, M.D., Torre, W., Garcia-Foncillas, J. and Montuenga, L.M. (2003) Altered patterns of expression of members of the heterogeneous nuclear ribonucleoprotein (hnRNP) family in lung cancer. Lung Cancer, 41, 131-143.

97. Carpenter, B., McKay, M., Dundas, S.R., Lawrie, L.C., Telfer, C. and Murray, G.I. (2006) Heterogeneous nuclear ribonucleoprotein $\mathrm{K}$ is over expressed, aberrantly localised and is associated with poor prognosis in colorectal cancer. Br J Cancer, 95, 921-927.

98. Chen, Y., Li, W. and Zhang, S. (2008) [hnRNP K expression and its clinical 
significance in human lung cancer tissues.]. Zhongguo Fei Ai Za Zhi, 11, 241-245.

99. Benelli, R., Monteghirfo, S., Balbi, C., Barboro, P. and Ferrari, N. (2009) Novel antivascular efficacy of metronomic docetaxel therapy in prostate cancer: hnRNP $\mathrm{K}$ as a player. Int J Cancer, 124, 2989-2996.

100. Chen, L.C., Chung, I.C., Hsueh, C., Tsang, N.M., Chi, L.M., Liang, Y., Chen, C.C., Wang, L.J. and Chang, Y.S. (2010) The antiapoptotic protein, FLIP, is regulated by heterogeneous nuclear ribonucleoprotein $\mathrm{K}$ and correlates with poor overall survival of nasopharyngeal carcinoma patients. Cell Death Differ, 17, 1463-1473.

101. Kimura, Y., Nagata, K., Suzuki, N., Yokoyama, R., Yamanaka, Y., Kitamura, H., Hirano, H. and Ohara, O. (2010) Characterization of multiple alternative forms of heterogeneous nuclear ribonucleoprotein $\mathrm{K}$ by phosphate-affinity electrophoresis. Proteomics, 10, 3884-3895.

102. Bomsztyk, K., Denisenko, O. and Ostrowski, J. (2004) hnRNP K: one protein multiple processes. Bioessays, 26, 629-638.

103. Du, Z., Fenn, S., Tjhen, R. and James, T.L. (2008) Structure of a construct of a human poly(C)-binding protein containing the first and second $\mathrm{KH}$ domains reveals insights into its regulatory mechanisms. J Biol Chem, 283, 28757-28766.

104. Du, Z., Yu, J., Chen, Y., Andino, R. and James, T.L. (2004) Specific recognition of the C-rich strand of human telomeric DNA and the RNA template of human telomerase by the first $\mathrm{KH}$ domain of human poly(C)-binding protein-2. $J$ Biol Chem, 279, 48126-48134.

105. Richard, M., Drouin, R. and Beaulieu, A.D. (1998) ABC50, a novel human ATP- 
binding cassette protein found in tumor necrosis factor-alpha-stimulated synoviocytes. Genomics, 53, 137-145.

106. Paytubi, S., Morrice, N.A., Boudeau, J. and Proud, C.G. (2008) The N-terminal region of $\mathrm{ABC} 50$ interacts with eukaryotic initiation factor eIF2 and is a target for regulatory phosphorylation by CK2. Biochem $J, 409,223-231$.

107. Paytubi, S., Wang, X., Lam, Y.W., Izquierdo, L., Hunter, M.J., Jan, E., Hundal, H.S. and Proud, C.G. (2009) ABC50 promotes translation initiation in mammalian cells. $J$ Biol Chem, 284, 24061-24073.

108. Early Breast Cancer Trialists' Collaborative Group (EBCTCG). (2005) Effects of chemotherapy and hormonal therapy for early breast cancer on recurrence and 15year survival: an overview of the randomised trials. Lancet, 365, 1687-1717.

109. Chan, M.Y., Rusconi, C.P., Alexander, J.H., Tonkens, R.M., Harrington, R.A. and Becker, R.C. (2008) A randomized, repeat-dose, pharmacodynamic and safety study of an antidote-controlled factor IXa inhibitor. $J$ Thromb Haemost, 6, 789796.

110. Keefe, A.D., Pai, S. and Ellington, A. (2010) Aptamers as therapeutics. Nat Rev Drug Discov, 9, 537-550.

111. Pan, W. and Clawson, G.A. (2009) The shorter the better: reducing fixed primer regions of oligonucleotide libraries for aptamer selection. Molecules, 14, 13531369.

112. Gyllensten, U.B. and Erlich, H.A. (1988) Generation of single-stranded DNA by the polymerase chain reaction and its application to direct sequencing of the HLA-DQA locus. Proc Natl Acad Sci U S A, 85, 7652-7656. 
113. Yang, Y., Yang, D., Schluesener, H.J. and Zhang, Z. (2007) Advances in SELEX and application of aptamers in the central nervous system. Biomol Eng, 24, 583592.

114. Vater, A., Jarosch, F., Buchner, K. and Klussmann, S. (2003) Short bioactive Spiegelmers to migraine-associated calcitonin gene-related peptide rapidly identified by a novel approach: tailored-SELEX. Nucleic Acids Res, 31, e130.

115. Pan, W., Xin, P. and Clawson, G.A. (2008) Minimal primer and primer-free SELEX protocols for selection of aptamers from random DNA libraries. Biotechniques, 44, 351-360.

116. Zimmermann, B., Bilusic, I., Lorenz, C. and Schroeder, R. (2010) Genomic SELEX: a discovery tool for genomic aptamers. Methods, 52, 125-132.

117. Lorenz, C., von Pelchrzim, F. and Schroeder, R. (2006) Genomic systematic evolution of ligands by exponential enrichment (Genomic SELEX) for the identification of protein-binding RNAs independent of their expression levels. Nat Protoc, 1, 2204-2212.

118. Mosing, R.K. and Bowser, M.T. (2009) Isolating aptamers using capillary electrophoresis-SELEX (CE-SELEX). Methods Mol Biol, 535, 33-43.

119. Mosing, R.K., Mendonsa, S.D. and Bowser, M.T. (2005) Capillary electrophoresis-SELEX selection of aptamers with affinity for HIV-1 reverse transcriptase. Anal Chem, 77, 6107-6112.

120. Mendonsa, S.D. and Bowser, M.T. (2004) In vitro evolution of functional DNA using capillary electrophoresis. J Am Chem Soc, 126, 20-21.

121. Lou, X., Qian, J., Xiao, Y., Viel, L., Gerdon, A.E., Lagally, E.T., Atzberger, P., 
Tarasow, T.M., Heeger, A.J. and Soh, H.T. (2009) Micromagnetic selection of aptamers in microfluidic channels. Proc Natl Acad Sci U SA, 106, 2989-2994.

122. Qian, J., Lou, X., Zhang, Y., Xiao, Y. and Soh, H.T. (2009) Generation of highly specific aptamers via micromagnetic selection. Anal Chem, 81, 5490-5495.

123. Nitsche, A., Kurth, A., Dunkhorst, A., Panke, O., Sielaff, H., Junge, W., Muth, D., Scheller, F., Stocklein, W., Dahmen, C. et al. (2007) One-step selection of Vaccinia virus-binding DNA aptamers by MonoLEX. BMC Biotechnol, 7, 48 .

124. Shamah, S.M., Healy, J.M. and Cload, S.T. (2008) Complex target SELEX. Acc Chem Res, 41, 130-138.

125. Cao, X., Li, S., Chen, L., Ding, H., Xu, H., Huang, Y., Li, J., Liu, N., Cao, W., Zhu, Y. et al. (2009) Combining use of a panel of ssDNA aptamers in the detection of Staphylococcus aureus. Nucleic Acids Res, 37, 4621-4628.

i26. Jeong, S., Han, S.R., Lee, Y.J. and Lee, S.W. (2010) Selection of RNA aptamers specific to active prostate-specific antigen. Biotechnol Lett, 32, 379-385.

127. White, R., Rusconi, C., Scardino, E., Wolberg, A., Lawson, J., Hoffman, M. and Sullenger, B. (2001) Generation of species cross-reactive aptamers using "toggle" SELEX. Mol Ther, 4, 567-573.

128. Shangguan, D., Li, Y., Tang, Z., Cao, Z.C., Chen, H.W., Mallikaratchy, P., Sefah, K., Yang, C.J. and Tan, W. (2006) Aptamers evolved from live cells as effective molecular probes for cancer study. Proc Natl Acad Sci US A, 103, 11838-11843.

129. Daniels, D.A., Chen, H., Hicke, B.J., Swiderek, K.M. and Gold, L. (2003) A tenascin-C aptamer identified by tumor cell SELEX: systematic evolution of ligands by exponential enrichment. Proc Natl Acad Sci U S A, 100, 15416-15421. 
130. Guo, K.T., Ziemer, G., Paul, A. and Wendel, H.P. (2008) CELL-SELEX: Novel perspectives of aptamer-based therapeutics. Int J Mol Sci, 9, 668-678.

131. Homann, M. and Goringer, H.U. (1999) Combinatorial selection of high affinity RNA ligands to live African trypanosomes. Nucleic Acids Res, 27, 2006-2014.

132. Gopinath, S.C., Kawasaki, K. and Kumar, P.K. (2005) Selection of RNA-aptamer against human influenza B virus. Nucleic Acids Symp Ser (Oxf), 85-86.

133. Kypr, J., Kejnovska, I., Renciuk, D. and Vorlickova, M. (2009) Circular dichroism and conformational polymorphism of DNA. Nucleic Acids Res, 37, 1713-1725.

134. Balagurumoorthy, P. and Brahmachari, S.K. (1994) Structure and stability of human telomeric sequence. $J$ Biol Chem, 269, 21858-21869.

135. Smargiasso, N., Gabelica, V., Damblon, C., Rosu, F., De Pauw, E., TeuladeFichou, M.P., Rowe, J.A. and Claessens, A. (2009) Putative DNA G-quadruplex formation within the promoters of Plasmodium falciparum var genes. $B M C$ Genomics, 10, 362.

136. Avci-Adali, M., Metzger, M., Perle, N., Ziemer, G. and Wendel, H.P. (2010) Pitfalls of cell-systematic evolution of ligands by exponential enrichment (SELEX): existing dead cells during in vitro selection anticipate the enrichment of specific aptamers. Oligonucleotides, 20, 317-323.

137. Paul, A., Avci-Adali, M., Ziemer, G. and Wendel, H.P. (2009) Streptavidin-coated magnetic beads for DNA strand separation implicate a multitude of problems during cell-SELEX. Oligonucleotides, 19, 243-254.

138. Zhao, Q., Matson, S., Herrera, C.J., Fisher, E., Yu, H. and Krieg, A.M. (1993) Comparison of cellular binding and uptake of antisense phosphodiester, 
phosphorothioate, and mixed phosphorothioate and methylphosphonate oligonucleotides. Antisense Res Dev, 3, 53-66.

139. Falcone, S., Cocucci, E., Podini, P., Kirchhausen, T., Clementi, E. and Meldolesi, J. (2006) Macropinocytosis: regulated coordination of endocytic and exocytic membrane traffic events. $J$ Cell Sci, 119, 4758-4769.

140. Stoltenburg, R., Reinemann, C. and Strehlitz, B. (2007) SELEX--a (r)evolutionary method to generate high-affinity nucleic acid ligands. Biomol Eng, 24, 381-403.

141. Sun, X. and Simon, A.E. (2003) Fitness of a turnip crinkle virus satellite RNA correlates with a sequence-nonspecific hairpin and flanking sequences that enhance replication and repress the accumulation of virions. $J$ Virol, 77, 78807889.

142. Sun, X., Zhang, G. and Simon, A.E. (2005) Short internal sequences involved in replication and virion accumulation in a subviral RNA of turnip crinkle virus. $J$ Virol, 79, 512-524.

143. Mi, J., Liu, Y., Rabbani, Z.N., Yang, Z., Urban, J.H., Sullenger, B.A. and Clary, B.M. (2010) In vivo selection of tumor-targeting RNA motifs. Nat Chem Biol, 6, 22-24.

144. Fiala, S. (1968) The cancer cell as a stem cell unable to differentiate. A theory of carcinogenesis. Neoplasma, 15, 607-622.

145. Jones, R.J., Matsui, W.H. and Smith, B.D. (2004) Cancer stem cells: are we missing the target? J Natl Cancer Inst, 96, 583-585.

146. Ganguly, R. and Puri, I.K. (2006) Mathematical model for the cancer stem cell hypothesis. Cell Prolif, 39, 3-14. 
147. Coghlin, C. and Murray, G.I. (2010) Current and emerging concepts in tumour metastasis. J Pathol, 222, 1-15.

148. Hedley, B.D. and Chambers, A.F. (2009) Tumor dormancy and metastasis. Adv Cancer Res, 102, 67-101.

149. McGowan, P.M., Kirstein, J.M. and Chambers, A.F. (2009) Micrometastatic disease and metastatic outgrowth: clinical issues and experimental approaches. Future Oncol, 5, 1083-1098.

150. Pantel, K., Alix-Panabieres, C. and Riethdorf, S. (2009) Cancer micrometastases. Nat Rev Clin Oncol, 6, 339-351.

151. Creighton, C.J., Li, X., Landis, M., Dixon, J.M., Neumeister, V.M., Sjolund, A., Rimm, D.L., Wong, H., Rodriguez, A., Herschkowitz, J.I. et al. (2009) Residual breast cancers after conventional therapy display mesenchymal as well as tumorinitiating features. Proc Natl Acad Sci U S A, 106, 13820-13825.

152. Jones, R.J. (2009) Cancer stem cells-clinical relevance. J Mol Med, 87, 11051110.

153. Huff, C.A., Matsui, W., Smith, B.D. and Jones, R.J. (2006) The paradox of response and survival in cancer therapeutics. Blood, 107, 431-434.

154. Lang, S.H., Frame, F.M. and Collins, A.T. (2009) Prostate cancer stem cells. $J$ Pathol, 217, 299-306.

155. Liu, Y., Clem, B., Zuba-Surma, E.K., El-Naggar, S., Telang, S., Jenson, A.B., Wang, Y., Shao, H., Ratajczak, M.Z., Chesney, J. et al. (2009) Mouse fibroblasts lacking RB1 function form spheres and undergo reprogramming to a cancer stem cell phenotype. Cell Stem Cell, 4, 336-347. 
156. Huntly, B.J., Shigematsu, H., Deguchi, K., Lee, B.H., Mizuno, S., Duclos, N., Rowan, R., Amaral, S., Curley, D., Williams, I.R. et al. (2004) MOZ-TIF2, but not $\mathrm{BCR}-\mathrm{ABL}$, confers properties of leukemic stem cells to committed murine hematopoietic progenitors. Cancer Cell, 6, 587-596.

157. Cozzio, A., Passegue, E., Ayton, P.M., Karsunky, H., Cleary, M.L. and Weissman, I.L. (2003) Similar MLL-associated leukemias arising from self-renewing stem cells and short-lived myeloid progenitors. Genes Dev, 17, 3029-3035.

158. Goldstein, A.S., Huang, J., Guo, C., Garraway, I.P. and Witte, O.N. (2010) Identification of a cell of origin for human prostate cancer. Science, 329, 568-571.

159. Lawson, D.A., Zong, Y., Memarzadeh, S., Xin, L., Huang, J. and Witte, O.N. (2010) Basal epithelial stem cells are efficient targets for prostate cancer initiation. Proc Natl Acad Sci U S A, 107, 2610-2615.

160. Hosonuma, S., Kobayashi, Y., Kojo, S., Wada, H., Seino, K., Kiguchi, K. and Ishizuka, B. (2011) Clinical significance of side population in ovarian cancer cells. Hum Cell, 24, 9-12.

161. Hurt, E.M., Kawasaki, B.T., Klarmann, G.J., Thomas, S.B. and Farrar, W.L. (2008) CD44+ CD24(-) prostate cells are early cancer progenitor/stem cells that provide a model for patients with poor prognosis. Br J Cancer, 98, 756-765.

162. Newton, T.C., Wolcott, K. and Roberts, S.S. (2010) Comparison of the side populations in pretreatment and postrelapse neuroblastoma cell lines. Transl Oncol, 3, 246-251.

163. Tsunoda, S., Okumura, T., Ito, T., Kondo, K., Ortiz, C., Tanaka, E., Watanabe, G., Itami, A., Sakai, Y. and Shimada, Y. (2006) ABCG2 expression is an independent 
unfavorable prognostic factor in esophageal squamous cell carcinoma. Oncology, $71,251-258$.

164. Kasper, S. (2009) Identification, characterization, and biological relevance of prostate cancer stem cells from clinical specimens. Urol Oncol, 27, 301-303.

165. Patrawala, L., Calhoun-Davis, T., Schneider-Broussard, R. and Tang, D.G. (2007) Hierarchical organization of prostate cancer cells in xenograft tumors: the CD44+alpha2beta1+ cell population is enriched in tumor-initiating cells. Cancer Res, 67, 6796-6805.

166. Al-Hajj, M., Wicha, M.S., Benito-Hernandez, A., Morrison, S.J. and Clarke, M.F. (2003) Prospective identification of tumorigenic breast cancer cells. Proc Natl Acad Sci U S A, 100, 3983-3988.

167. Miki, J., Furusato, B., Li, H., Gu, Y., Takahashi, H., Egawa, S., Sesterhenn, I.A., McLeod, D.G., Srivastava, S. and Rhim, J.S. (2007) Identification of putative stem cell markers, CD133 and CXCR4, in hTERT-immortalized primary nonmalignant and malignant tumor-derived human prostate epithelial cell lines and in prostate cancer specimens. Cancer Res, 67, 3153-3161.

168. Vander Griend, D.J., Karthaus, W.L., Dalrymple, S., Meeker, A., DeMarzo, A.M. and Isaacs, J.T. (2008) The role of CD133 in normal human prostate stem cells and malignant cancer-initiating cells. Cancer Res, 68, 9703-9711.

169. Trerotola, M., Rathore, S., Goel, H.L., Li, J., Alberti, S., Piantelli, M., Adams, D., Jiang, Z. and Languino, L.R. (2010) CD133, Trop-2 and alpha2betal integrin surface receptors as markers of putative human prostate cancer stem cells. $A m J$ Transl Res, 2, 135-144. 
170. Goldstein, A.S., Lawson, D.A., Cheng, D., Sun, W., Garraway, I.P. and Witte, O.N. (2008) Trop2 identifies a subpopulation of murine and human prostate basal cells with stem cell characteristics. Proc Natl Acad Sci U S A, 105, 20882-20887.

171. Challen, G.A. and Little, M.H. (2006) A side order of stem cells: the SP phenotype. Stem Cells, 24, 3-12.

172. Liu, W.H., Qian, N.S., Li, R. and Dou, K.F. (2010) Replacing Hoechst33342 with rhodamine 123 in isolation of cancer stem-like cells from the MHCC 97 cell line. Toxicol In Vitro, 24, 538-545.

173. Szotek, P.P., Pieretti-Vanmarcke, R., Masiakos, P.T., Dinulescu, D.M., Connolly, D., Foster, R., Dombkowski, D., Preffer, F., Maclaughlin, D.T. and Donahoe, P.K. (2006) Ovarian cancer side population defines cells with stem cell-like characteristics and Mullerian Inhibiting Substance responsiveness. Proc Natl Acad Sci U S A, 103, 11154-11159.

174. Chiba, T., Kita, K., Zheng, Y.W., Yokosuka, O., Saisho, H., Iwama, A., Nakauchi, H. and Taniguchi, H. (2006) Side population purified from hepatocellular carcinoma cells harbors cancer stem cell-like properties. Hepatology, 44, 240251.

175. Ho, M.M., Ng, A.V., Lam, S. and Hung, J.Y. (2007) Side population in human lung cancer cell lines and tumors is enriched with stem-like cancer cells. Cancer Res, 67, 4827-4833.

176. Mitsutake, N., Iwao, A., Nagai, K., Namba, H., Ohtsuru, A., Saenko, V. and Yamashita, S. (2007) Characterization of side population in thyroid cancer cell lines: cancer stem-like cells are enriched partly but not exclusively. 
Endocrinology, 148, 1797-1803.

177. Mimeault, M. and Batra, S.K. (2009) Characterization of nonmalignant and malignant prostatic stem/progenitor cells by Hoechst side population method. Methods Mol Biol, 568, 139-149.

178. Zhang, S.N., Huang, F.T., Huang, Y.J., Zhong, W. and Yu, Z. (2010) Characterization of a cancer stem cell-like side population derived from human pancreatic adenocarcinoma cells. Tumori, 96, 985-992.

179. Salcido, C.D., Larochelle, A., Taylor, B.J., Dunbar, C.E. and Varticovski, L. (2010) Molecular characterisation of side population cells with cancer stem celllike characteristics in small-cell lung cancer. Br J Cancer, 102, 1636-1644.

180. van den Hoogen, C., van der Horst, G., Cheung, H., Buijs, J.T., Lippitt, J.M., Guzman-Ramirez, N., Hamdy, F.C., Eaton, C.L., Thalmann, G.N., Cecchini, M.G. et al. (2010) High aldehyde dehydrogenase activity identifies tumor-initiating and metastasis-initiating cells in human prostate cancer. Cancer Res, 70, 5163-5173.

181. Yu, C., Yao, Z., Dai, J., Zhang, H., Escara-Wilke, J., Zhang, X. and Keller, E.T. (2011) ALDH activity indicates increased tumorigenic cells, but not cancer stem cells, in prostate cancer cell lines. In Vivo, 25, 69-76.

182. Charafe-Jauffret, E., Ginestier, C., Iovino, F., Tarpin, C., Diebel, M., Esterni, B., Houvenaeghel, G., Extra, J.M., Bertucci, F., Jacquemier, J. et al. (2010) Aldehyde dehydrogenase 1-positive cancer stem cells mediate metastasis and poor clinical outcome in inflammatory breast cancer. Clin Cancer Res, 16, 45-55.

183. Lee, H.E., Kim, J.H., Kim, Y.J., Choi, S.Y., Kim, S.W., Kang, E., Chung, I.Y., Kim, I.A., Kim, E.J., Choi, Y. et al. (2011) An increase in cancer stem cell 
population after primary systemic therapy is a poor prognostic factor in breast cancer. BrJ Cancer, 104, 1730-1738.

184. Silva, I.A., Bai, S., McLean, K., Yang, K., Griffith, K., Thomas, D., Ginestier, C., Johnston, C., Kueck, A., Reynolds, R.K. et al. (2011) Aldehyde Dehydrogenase in Combination with CD133 Defines Angiogenic Ovarian Cancer Stem Cells That Portend Poor Patient Survival. Cancer Res, 71, 3991-4001.

185. Li, T., Su, Y., Mei, Y., Leng, Q., Leng, B., Liu, Z., Stass, S.A. and Jiang, F. (2010) ALDH1A1 is a marker for malignant prostate stem cells and predictor of prostate cancer patients' outcome. Lab Invest, 90, 234-244.

186. Dontu, G., Abdallah, W.M., Foley, J.M., Jackson, K.W., Clarke, M.F., Kawamura, M.J. and Wicha, M.S. (2003) In vitro propagation and transcriptional profiling of human mammary stem/progenitor cells. Genes Dev, 17, 1253-1270.

187. Hemmati, H.D., Nakano, I., Lazareff, J.A., Masterman-Smith, M., Geschwind, D.H., Bronner-Fraser, M. and Kornblum, H.I. (2003) Cancerous stem cells can arise from pediatric brain tumors. Proc Natl Acad Sci U S A, 100, 15178-15183.

188. Svendsen, C.N., ter Borg, M.G., Armstrong, R.J., Rosser, A.E., Chandran, S., Ostenfeld, T. and Caldwell, M.A. (1998) A new method for the rapid and long term growth of human neural precursor cells. J Neurosci Methods, 85, 141-152.

189. Miki, J. and Rhim, J.S. (2008) Prostate cell cultures as in vitro models for the study of normal stem cells and cancer stem cells. Prostate Cancer Prostatic Dis, $11,32-39$.

190. Rybak, A.P., He, L., Kapoor, A., Cutz, J.C. and Tang, D. (2011) Characterization of sphere-propagating cells with stem-like properties from DU145 prostate cancer 
cells. Biochim Biophys Acta, 1813, 683-694.

191. Fan, X., Liu, S., Su, F., Pan, Q. and Lin, T. (2010) Effective enrichment of prostate cancer stem cells from spheres in a suspension culture system. Urol Oncol.

192. Duhagon, M.A., Hurt, E.M., Sotelo-Silveira, J.R., Zhang, X. and Farrar, W.L. (2010) Genomic profiling of tumor initiating prostatospheres. BMC Genomics, 11, 324.

193. Kallifatidis, G., Labsch, S., Rausch, V., Mattern, J., Gladkich, J., Moldenhauer, G., Buchler, M.W., Salnikov, A.V. and Herr, I. (2011) Sulforaphane increases drugmediated cytotoxicity toward cancer stem-like cells of pancreas and prostate. $\mathrm{Mol}$ Ther, 19, 188-195.

194. Luk, S.U., Yap, W.N., Chiu, Y.T., Lee, D.T., Ma, S., Lee, T.K., Vasireddy, R.S., Wong, Y.C., Ching, Y.P., Nelson, C. et al. (2011) Gamma-tocotrienol as an effective agent in targeting prostate cancer stem cell-like population. Int $J$ Cancer, 128, $2182-2191$.

195. Joseph, I., Tressler, R., Bassett, E., Harley, C., Buseman, C.M., Pattamatta, P., Wright, W.E., Shay, J.W. and Go, N.F. (2010) The telomerase inhibitor imetelstat depletes cancer stem cells in breast and pancreatic cancer cell lines. Cancer Res, 70, 9494-9504.

196. Gupta, P.B., Onder, T.T., Jiang, G., Tao, K., Kuperwasser, C., Weinberg, R.A. and Lander, E.S. (2009) Identification of selective inhibitors of cancer stem cells by high-throughput screening. Cell, 138, 645-659.

197. Levina, V., Marrangoni, A.M., DeMarco, R., Gorelik, E. and Lokshin, A.E. (2008) 
Drug-selected human lung cancer stem cells: cytokine network, tumorigenic and metastatic properties. PLoS One, 3, e3077.

198. Calcagno, A.M., Salcido, C.D., Gillet, J.P., Wu, C.P., Fostel, J.M., Mumau, M.D., Gottesman, M.M., Varticovski, L. and Ambudkar, S.V. (2010) Prolonged drug selection of breast cancer cells and enrichment of cancer stem cell characteristics. J Natl Cancer Inst, 102, 1637-1652.

199. Broadley, K.W., Hunn, M.K., Farrand, K.J., Price, K.M., Grasso, C., Miller, R.J., Hermans, I.F. and McConnell, M.J. (2011) Side population is not necessary or sufficient for a cancer stem cell phenotype in glioblastoma multiforme. Stem Cells, 29, 452-461.

200. Awad, O., Yustein, J.T., Shah, P., Gul, N., Katuri, V., O'Neill, A., Kong, Y., Brown, M.L., Toretsky, J.A. and Loeb, D.M. (2010) High ALDH activity identifies chemotherapy-resistant Ewing's sarcoma stem cells that retain sensitivity to EWS-FLI1 inhibition. PLoS One, 5, e13943.

201. Patrawala, L., Calhoun, T., Schneider-Broussard, R., Zhou, J., Claypool, K. and Tang, D.G. (2005) Side population is enriched in tumorigenic, stem-like cancer cells, whereas $\mathrm{ABCG} 2+$ and $\mathrm{ABCG} 2-$ cancer cells are similarly tumorigenic. Cancer Res, 65, 6207-6219.

202. Moon, T.E., Salmon, S.E., White, C.S., Chen, H.S., Meyskens, F.L., Durie, B.G. and Alberts, D.S. (1981) Quantitative association between the in vitro human tumor stem cell assay and clinical response to cancer chemotherapy. Cancer Chemother Pharmacol, 6, 211-218. 
APPENDIX A

Manuscript from Nucleic Acids Research 


\title{
Cancer-selective antiproliferative activity is a general property of some G-rich oligodeoxynucleotides
}

\author{
Enid W. Choi ${ }^{1}$, Lalitha V. Nayak ${ }^{2}$ and Paula J. Bates ${ }^{* 1,2}$ \\ ${ }^{1}$ Department of Biochemistry and Molecular Biology and ${ }^{2}$ Department of Medicine, James Graham Brown \\ Cancer Center, University of Louisville, Louisville, KY, USA
}

Received June 9, 2009; Revised October 23, 2009; Accepted November 8, 2009

\begin{abstract}
Oligodeoxynucleotide libraries containing randomly incorporated bases are used to generate DNA aptamers by systematic evolution of ligands by exponential enrichment (SELEX). We predicted that combinatorial libraries with alternative base compositions might have innate properties different from the standard library containing equimolar $A+C+G+T$ bases. In particular, we hypothesized that G-rich libraries would contain a higher proportion of quadruplex-forming sequences, which may impart desirable qualities, such as increased nuclease resistance and enhanced cellular uptake. Here, we report on 11 synthetic oligodeoxynucleotide libraries of various base combinations and lengths, with regard to their circular dichroism, stability in serum-containing medium, cellular uptake, protein binding and antiproliferative activity. Unexpectedly, we found that some G-rich libraries (composed of $\mathbf{G}+\mathbf{T}$ or $\mathbf{G}+\mathbf{C}$ nucleotides) strongly inhibited cancer cell growth while sparing non-malignant cells. These libraries had spectral features consistent with G-quadruplex formation, were significantly more stable in serum than inactive libraries and showed enhanced cellular uptake. Active libraries generally had strong protein binding, while the pattern of protein binding suggested that $G / T$ and $G / C$ libraries have distinct mechanisms of action. In conclusion, cancer-selective antiproliferative activity may be a general feature of certain G-rich oligodeoxynucleotides and is associated with quadruplex formation, nuclease resistance, efficient cellular uptake and protein binding.
\end{abstract}

\section{INTRODUCTION}

Cancer is a leading cause of death throughout the world. Although many drugs have been developed to treat cancer, the majority of the oncology pharmacopeia causes serious, dose-limiting side effects due to damage to healthy cells. There exists a clear need for better, more specific cancer therapies, not only to increase efficacy against cancer cells but also to decrease the suffering of patients who are subjected to these drug regimens.

Nucleic acid aptamers are emerging as a new class of targeted therapeutic agents and are the focus of a rapidly growing field of medical research (1-4). These small synthetic oligomers of DNA or RNA form stable three-dimensional structures and bind to defined molecular targets via shape-specific recognition. Thus, in essence, their mechanism of action is similar to that of protein-based monoclonal antibodies. However, due to their smaller size and different chemical composition, aptamers have a number of potential advantages over antibodies. These include ease of manufacture and storage, facile conjugation, better tumor penetration, more rapid systemic clearance and non-immunogenicity. In recent years, aptamers have been identified as potential treatments for a variety of diseases, including macular degeneration $(5,6)$, autoimmune disease (7), disseminated intravascular coagulation $(8,9)$ and prion diseases $(10)$. In the oncology field, the most clinically advanced aptamer, and the first anticancer aptamer to be tested in humans, is a 26-nt G-rich DNA known as AS1411 (formerly, AGRO100). This nucleolin-targeted aptamer, which was discovered by our group, has shown promising clinical activity without any major side effects in Phase I and ongoing Phase II clinical trials (11).

New aptamers against specific targets are most often engineered via systematic evolution of ligands by exponential enrichment (SELEX) $(1,12,13)$, an iterative methodology designed to create target-specific aptamers from a

\footnotetext{
*To whom correspondence should be addressed. Tel: +1 502852 2432; Fax: + 1502852 2356; Email: paula.bates(alouisville.edu

The Author(s) 2009. Published by Oxford University Press.

This is an Open Access article distributed under the terms of the Creative Commons Attribution Non-Commercial License (http:/creativecommons.org/licenses/ by-nc/2.5), which permits unrestricted non-commercial use. distribution, and reproduction in any medium, provided the original work is properly cited.
} 


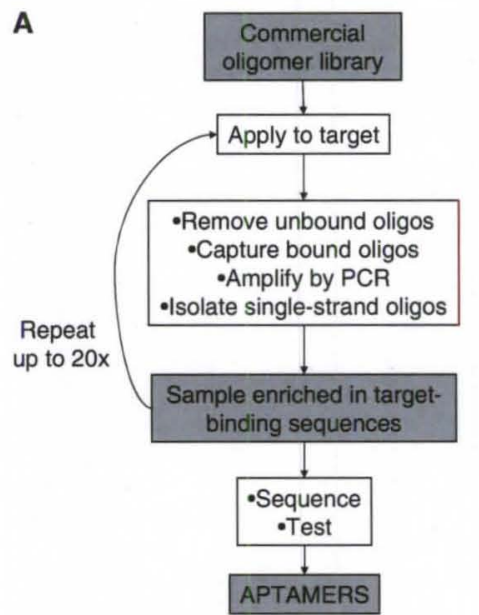

B

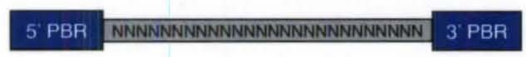

$\mathrm{N}=\mathrm{A}, \mathrm{C}, \mathrm{G}$ or $\mathrm{T}$

possible sequences $=4^{26} \approx 4 \times 10^{15}$

C

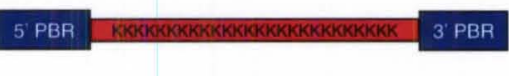

$\mathrm{K}=\mathrm{G}$ or $\mathrm{T}$

possible sequences $=2^{26} \approx 7 \times 10^{7}$

Figure 1. Overview of SELEX procedure and oligonucleotide libraries. (A) Schematic of SELEX procedure. (B) Diagram of N library. Primer-binding regions (PBR) flank a length of random sequence, for which each base has equal likelihood of being an A, C, G or T. In theory, the library contains $4^{n}$ different sequences, where $n$ is the length of the random sequence (in this example $n=26$ ). (C) Example of a guanine-rich GT library. The random sequence is restricted such that each base may be either a G or a T. This library should contain $2^{n}$ different sequences (in this example $n=26$ ).

combinatorial library (Figure 1A). For DNA aptamers, a single-stranded oligodeoxynucleotide library consisting of two known primer-binding sequences flanking a random sequence of usually $20-40 \mathrm{nt}$ in length (N library, where $\mathrm{N}=\mathrm{A}, \mathrm{C}, \mathrm{G}$, or T; Figure 1B) is applied to the target of interest. The molecules that do not bind are washed away, and the remainder are harvested and amplified by polymerase chain reaction (PCR). The aptamer-enriched pool of single-stranded molecules is then isolated from the PCR product, reapplied to the target and the process is repeated. In this manner, highly specific aptamer sequences are usually evolved after $10-20$ cycles. For RNA aptamers, a similar procedure with additional steps for transcription and reverse transcription can be utilized. Typically, the target of interest for SELEX is a purified protein or small molecule, but the development of aptamers against intact cells or organisms can be also achieved $(1,14)$.

One shortcoming of traditional SELEX is the high number of iterations required, which consumes time and resources, and increases the likelihood of artifacts (since every round of SELEX requires PCR and must be carefully protected from contamination). Moreover, for in vivo use, evolved sequences must typically be modified to enhance their pharmacokinetic properties and to increase resistance to serum nucleases. This can be achieved post-SELEX, or by incorporating modified bases or backbones during the SELEX procedure $(1,15)$. However, these approaches have some drawbacks: post-SELEX modification can reduce aptamer activity or increase toxicity, and not all modified nucleotides are compatible with the polymerases used during SELEX. An additional shortcoming of aptamers (which is also shared by antibodies) is their inefficient cellular internalization, which generally limits their clinical targets to extracellular or cell surface molecules.
In an attempt to address some of these limitations, we have considered the use of alternative starting libraries for SELEX. Rather than the random N library containing billions of sequences, the vast majority of which will not turn out to be clinically useful aptamers, we propose beginning with smaller libraries, especially those which may be enriched in molecules that contain secondary structure motifs, such as hairpins or G-quartets. In particular, we hypothesized that libraries enriched in guanine (G) residues (for example, see Figure 1C) would contain a higher proportion of quadruplex-forming sequences, which may impart increased functionality, nuclease resistance and cellular uptake compared to the $\mathrm{N}$ library. An additional driving force for this study was the realization that very G-rich sequences such as AS1411 (5'-d[GGTGG TGGTGGTTGTGGTGGTGGTGG]), which was discovered by chance, would rarely occur in the standard $\mathrm{N}$ library, making it unlikely to have been discovered by standard SELEX. Furthermore, our previous work involving the 'bench-to-bedside' development of AS1411 and the preclinical research of several other groups who are researching a variety of different G/T-containing oligonucleotides (11) led us to focus on G/T DNA libraries for our studies on the effect of oligonucleotide length.

As a prelude to SELEX studies using alternative oligodeoxynucleotide libraries, we have sought to establish the baseline characteristics of various libraries and to test our hypothesis that G-rich libraries will be enriched in quadruplex-forming sequences with improved nuclease resistance and cellular uptake. In this project, we have examined 11 oligodeoxynucleotide libraries of varying base compositions and lengths, as well as three monobasic oligomers (Figure 2). We have performed studies to evaluate various characteristics of the libraries, including a well-established colorimetric assay to determine their 


\begin{tabular}{|c|c|c|c|c|c|c|}
\hline & Name & Length & $\% \mathbf{A}$ & $\% \mathrm{C}$ & $\% \mathrm{G}$ & $\% \mathrm{~T}$ \\
\hline \multirow{6}{*}{$\begin{array}{l}\frac{5}{0} \\
\frac{1}{2} \\
\frac{1}{1} \\
\frac{1}{0} \\
z\end{array}$} & N 26mer & 26 & 25 & 25 & 25 & 25 \\
\hline & A 26mer & 26 & 100 & & & \\
\hline & C 26 mer & 26 & & 100 & & \\
\hline & T 26mer & 26 & & & & 100 \\
\hline & AT 26mer & 26 & 50 & & . & 50 \\
\hline & CT $26 \mathrm{mer}$ & 26 & & 50 & & 50 \\
\hline \multirow{8}{*}{$\frac{\frac{5}{2}}{\frac{1}{5}}$} & GT 10mer & 10 & & & 50 & 50 \\
\hline & GT 18mer & 18 & & & 50 & 50 \\
\hline & GT 26mer & 26 & & & 50 & 50 \\
\hline & GT 34mer & 34 & & & 50 & 50 \\
\hline & GT $0.3326 \mathrm{mer}$ & 26 & & & 33 & 67 \\
\hline & GT $0.6726 \mathrm{mer}$ & 26 & & & 67 & 33 \\
\hline & GA 26mer & 26 & 50 & & 50 & \\
\hline & GC 26mer & 26 & & 50 & 50 & \\
\hline
\end{tabular}

Figure 2. Oligonucleotides and libraries used in this study. Singlestranded DNA oligonucleotide libraries and monobasic oligomers used in this study. Libraries with $>25 \%$ guanines are considered 'G-rich'.

intrinsic antiproliferative activities in three different cancer cell lines compared to non-malignant cells. As far as we know, we are the first to perform such a comprehensive screen of oligonucleotide libraries. In addition, we report here the unexpected discovery that certain G-rich libraries have high levels of intrinsic, cancer-selective antiproliferative activity.

\section{MATERIALS AND METHODS}

Oligodeoxynucleotides and libraries

All oligonucleotides and oligonucleotide libraries were purchased from Integrated DNA Technologies, Inc. (Coralville, IA, USA) and are shown in Figure 2. The relative proportion of bases was specified for libraries, which can be synthesized by mixing phosphoramidite precursors in the desired ratio. The specified bases are then randomly incorporated into the sequence. Lyophilized libraries were resuspended in filter-sterilized water, then heated at $65^{\circ} \mathrm{C}$ for $10 \mathrm{~min}$ and vortexed vigorously to dissolve. For uptake studies, three thymidine bases (TTT) were added to the 3 -end of the library sequence to avoid quenching the 3 -fluorophore, which can occur when it is proximal to a G-quartet (Choi,E., Nayak,L. and Bates,P. unpublished data). $\mathrm{A}_{26}$ oligodeoxynucleotide was originally proposed for study, but is not commercially available due to difficulties in synthesis of oligodeoxyguanosines of this length.

\section{Cell culture}

Four cell lines were comprehensively studied: A549 lung adenocarcinoma, DU145 prostate adenocarcinoma, MCF7 mammary carcinoma and Hs27 non-malignant skin fibroblasts. All cell lines were acquired from American Type Culture Collection (ATCC, Manassas, VA, USA). Cells were grown in complete cell culture medium of Dulbecco's Modified Eagle Medium
(DMEM, Invitrogen, Carlsbad, CA, USA) supplemented with $10 \%$ heat-treated $\left(15 \mathrm{~min}\right.$ at $\left.65^{\circ} \mathrm{C}\right)$ fetal bovine serum (FBS, Invitrogen) and $1 \%$ penicillin/streptomycin (Invitrogen). The MCF7 cell line alone was grown using charcoal-stripped serum (Invitrogen). All cells were cultured at $37^{\circ} \mathrm{C}$ in $5 \% \mathrm{CO}_{2}$. Methods for cell culture and antiproliferative assays using MCF10A cells can be found in the Supplementary Data.

\section{Antiproliferative activity assay}

In the four cells lines, each library/oligomer was tested in triplicate at eight concentrations of oligonucleotide $(0,2,4,6,8,10,12$ and $14 \mu \mathrm{M})$ using a modified previously published MTT [3-(4,5-dimethylthiazol-2-yl)2,5-diphenyltetrazolium bromide] assay protocol (16). Briefly, cells were seeded in 96-well tissue culture plates and incubated overnight. To account for intrinsic differences in growth rate, the A549 and DU145 lines were plated at 1000 cells per well, MCF7 at 2000 per well and Hs27 at 3000 per well. Oligomer library stock solutions were then added to each well to yield the desired final concentration. Plates were incubated with the oligomer libraries for 5 days, during which the cell culture medium was not changed. On Day 5, cell viability was determined and the background corresponding to medium alone (no cells) was subtracted. For each concentration, the mean and standard error of triplicate wells was plotted. Assays were repeated at least twice in triplicate to ensure reproducibility of the results.

\section{Circular dichroism spectroscopy}

Oligomer library stocks were diluted to a concentration of $5 \mu \mathrm{M}$ (strand concentration) using either water (buffer was not included in order to be representative of the stock solutions used to treat cells), or aqueous solutions containing $100 \mathrm{mM}$ sodium chloride or $100 \mathrm{mM}$ potassium chloride. Samples were incubated at $95^{\circ} \mathrm{C}$ for $5 \mathrm{~min}$, then cooled on ice until analysis. Circular dichroism (CD) spectra were collected at $20^{\circ} \mathrm{C}$ using a Jasco J-715 spectropolarimeter between 340 and $220 \mathrm{~nm}$ wavelengths, using $200 \mathrm{~nm} / \mathrm{min}$ scanning speed, $2 \mathrm{~s}$ response time, $5 \mathrm{~nm}$ bandwidth and 1-cm pathlength. For each set of samples, the same solution used to prepare the oligomers was used as the reference. The resulting spectra in millidegrees (mdeg) were converted to molar $\mathrm{CD}(\Delta \varepsilon)$, referring to per mole of nucleotide. Methods for analysis of $(\mathrm{dC})_{26}$ at various $\mathrm{pH}$ can be found in the Supplementary Data.

\section{Nuclease-resistance in serum-containing medium}

Each oligomer library was $5^{\prime}$-end-labeled with ${ }^{32} \mathrm{P}$ using T4 kinase (Invitrogen) and unincorporated $\gamma-{ }^{32} \mathrm{P}-\mathrm{ATP}$ was removed from samples using TE-Midi SELECT-D G-25 columns (Shelton Scientific, Shelton, CT, USA), according to manufacturers' directions. Collection tubes containing end-labeled libraries were kept on ice, or stored at $-20^{\circ} \mathrm{C}$. The radioactivity of end-labeled libraries was measured in a scintillation counter on the day of the experiment. For each library to be tested, four reaction tubes containing complete cell culture medium without antibiotics were 
prepared. One million counts per minute of end-labeled oligomer was added to each of four tubes. Samples were incubated at $37^{\circ} \mathrm{C}$, and tubes were flash-frozen in a dry ice/ethanol bath at $0,1.5,24$ and $72 \mathrm{~h}$. Frozen samples were stored at $-80^{\circ} \mathrm{C}$ until all samples had been harvested. Samples were then thawed on ice and run on $12 \%$ denaturing polyacrylamide gels. Band density was measured over each lane using the UN-SCAN-IT gel densitometry application (Silk Scientific, Orem, UT, USA). The percent sample remaining was calculated taking the signal of the zero time point lane to be $100 \%$.

\section{Cellular uptake}

Uptake experiments were performed using oligomer libraries labeled at the $3^{\prime}$ end with FAM (6-carboxyfluorescein) for representative libraries that were inactive (N 26mer and GA 26mer) or active (GC $26 \mathrm{mer}$ and GT 26mer). For each sample, $3 \times 10^{5}$ cells were plated and incubated overnight. The following day, fresh medium was added to each plate. FAM-labeled libraries were added to make a final concentration of $5-\mu \mathrm{M}$ oligonucleotide and plates were incubated for $1 \mathrm{~h}$. The medium containing unbound oligo was removed. Cells were washed twice and treated with trypsin (Invitrogen) to remove surface-bound oligonucleotides and harvest cells. Cells were kept on ice and protected from light until analysis. Propidium iodide (PI, $2 \mu \mathrm{g}$ per sample, BD Biosciences, San Jose, CA, USA) was added as a marker of viability. Ten thousand cells were counted, after gating to exclude PI-positive (non-viable) cells from analysis. Cells were analyzed using a FACSCalibur cytometer (BD Biosciences). The relative fluorescence of cells was determined using the FlowJo software program (Tree Star, Ashland, OR, USA).

\section{Protein binding using electrophoretic mobility shift assay}

Libraries were end-labeled with ${ }^{32} \mathrm{P}$ as described for the nuclease-resistance assay and then electrophoresed on a non-denaturing $20 \%$ polyacrylamide gel to check labeling. Whole-cell extracts from A549, DU145, MCF7 and Hs27 cells were prepared using a modification of a previously published method (17) as follows: cells were grown until confluent in $75 \mathrm{~cm}^{2}$ flasks. The cells were washed, then scraped with $100 \mu \mathrm{l}$ HEDG buffer [20 mM HEPES-NaOH, pH 7.8, 0.5 mM EDTA, $0.5 \mathrm{mM}$ dithiothreitol (DTT)] containing $10 \%$ glycerol, $1 \times$ protease inhibitor cocktail (Sigma, St Louis), and $0.42 \mathrm{M} \mathrm{NaCl}$. The solution was harvested and subjected to three freeze-thaw cycles (liquid nitrogen versus room temperature). Samples were centrifuged at $16000 \mathrm{~g}$ at $4^{\circ} \mathrm{C}$ for $45 \mathrm{~min}$ to pellet cell debris. An equal volume of HEDG with glycerol and protease inhibitor but without $\mathrm{NaCl}$ was added to reach a final concentration of $0.21 \mathrm{M} \mathrm{NaCl}$. An aliquot (100000 c.p.m.) of radiolabeled oligomer was combined with $5.0 \mu \mathrm{g}$ of cell extract from A549, DU145 and MCF7 cells, or with $3.2 \mu \mathrm{g}$ from Hs27 cells, and $5 \mathrm{X}$ protein binding buffer [ $1 \mathrm{X}$ is $20 \mathrm{mM}$ Tris $-\mathrm{HCl}, \mathrm{pH} 7.4$, $140 \mathrm{mM} \mathrm{Kl}, 2.5 \mathrm{mM} \mathrm{MgCl} 2,1 \mathrm{mM}$ dithiothreitol, $0.2 \mathrm{mM}$ phenylmethylsulfonyl fluoride (PMSF), $8 \%$ (v/v) glycerol]. Samples were incubated at $37^{\circ} \mathrm{C}$ for $15 \mathrm{~min}$ and run on a non-denaturing 5\% polyacrylamide gel. Gels were fixed in a solution of $10 \%(\mathrm{v} / \mathrm{v})$ methanol $+10 \%$ $(\mathrm{v} / \mathrm{v})$ glacial acetic acid in water for $10 \mathrm{~min}$ at room temperature, then exposed to a storage phosphor screen for $2 \mathrm{~h}$ at room temperature before scanning. Gels were also exposed to X-ray film overnight at $-80^{\circ} \mathrm{C}$.

\section{RESULTS AND DISCUSSION}

GT libraries of all types and the GC $26 \mathrm{mer}$ library have selective antiproliferative activity against cancer cells

The antiproliferative activity of 11 oligomer libraries and three monobasic oligomers at various concentrations against four cell lines was determined using the MTT assay. The results are summarized in Figure $3 \mathrm{~A}$, which shows the percentage reduction in cell number at $6-\mu \mathrm{M}$ oligonucleotide strand concentration. This format was selected for clarity of presentation and $6 \mu \mathrm{M}$ was chosen because it best illustrated the relative activities of the various libraries.

Some libraries containing $\mathrm{G}+\mathrm{T}$ or $\mathrm{G}+\mathrm{C}$ were found to strongly inhibit cancer cell proliferation at low micromolar concentrations, whereas non-G-rich libraries, including the standard $\mathrm{N}$ library, had little effect on the cancer cells (Figure 3A). All libraries tested had minimal antiproliferative activity against the non-cancerous $\mathrm{Hs} 27$ cells (Figure 3A and B). These novel data provide surprising evidence that some oligomer libraries possess intrinsic antiproliferative activity, even before isolation of individual aptamer sequences.

The GT 18-mer, 26-mer and 34-mer libraries had the highest level of antiproliferative activity against the three cancer cell lines (Figure 3A). The GT 10-mer, GT 0.33 26-mer and GT 0.67 26-mer libraries had similar specificity, but were less effective at inhibiting the cancer cells (Figure 3A). Figure 3B and C illustrate these results for A549 cells, and similar patterns of activity were observed in the other cancer cell lines (Figure 3A and data not shown). The GC 26-mer library had potent antiproliferative activity against A549 and DU145 cells, and had a weaker effect on MCF7 cells (Figure 3E). In contrast to the other G-rich libraries, the GA 26-mer library had very little antiproliferative activity against any cell line (Figure 3A and data not shown). Based on the shape of the dose-dependence curves (for example, see Figure $3 \mathrm{C}$, which plateaus at a non-zero value), it appears that active libraries have a cytostatic rather than cytotoxic effect. This was also observed previously for individual antiproliferative G-rich oligonucleotides (16).

From these data, we designated all of the GT libraries and the GC 26-mer library as 'active' against cancer cells. In the remaining experiments, we compared these active libraries against the remaining 'inactive' libraries in order to identify characteristics that were associated with the unexpected antiproliferative activity. To confirm the cancer-selectivity of the active libraries, we also examined all libraries and oligomers in an additional non-tumorigenic cell line, MCF10A, which is derived from benign breast epithelial cells. The results indicated 
A Percent Inhibition in Cell Number at $6 \mu \mathrm{M}$ Concentration

\begin{tabular}{|c|c|c|c|c|c|}
\hline & Library & $\begin{array}{c}\text { A549 } \\
\text { lung cancer }\end{array}$ & $\begin{array}{c}\text { DU145 } \\
\text { prostate cancer }\end{array}$ & $\begin{array}{c}\text { MCF7 } \\
\text { breast cancer }\end{array}$ & $\begin{array}{c}\text { Hs27 } \\
\text { non-malignant }\end{array}$ \\
\hline \multirow{6}{*}{ 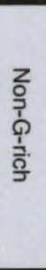 } & N 26mer & 0 & 6 & 27 & 11 \\
\hline & A 26mer & 0 & 0 & 2 & 6 \\
\hline & C 26mer & 0 & 0 & 1 & 13 \\
\hline & T 26mer & 0 & 7 & 24 & 14 \\
\hline & AT 26mer & 0 & 28 & 21 & 18 \\
\hline & СТ 26mer & 4 & 10 & 10 & 21 \\
\hline \multirow{8}{*}{$\begin{array}{l}\text { p) } \\
\frac{1}{3}\end{array}$} & GT 10mer & 37 & 35 & 53 & 6 \\
\hline & GT $18 \mathrm{mer}$ & 63 & 58 & 63 & 6 \\
\hline & GT 26mer & 72 & 60 & 69 & 11 \\
\hline & GT 34mer & 71 & 68 & 63 & 9 \\
\hline & GT $0.3326 \mathrm{mer}$ & 47 & 29 & 53 & 14 \\
\hline & GT $0.6726 \mathrm{mer}$ & 47 & 27 & 51 & 5 \\
\hline & GA 26mer & 0 & 18 & 18 & 4 \\
\hline & GC 26mer & 70 & 72 & 31 & 3 \\
\hline
\end{tabular}

\section{B Hs27}

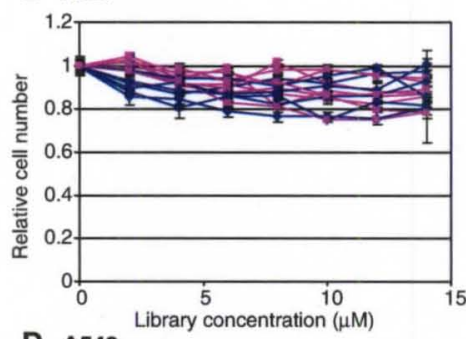

D $\mathbf{A 5 4 9}$

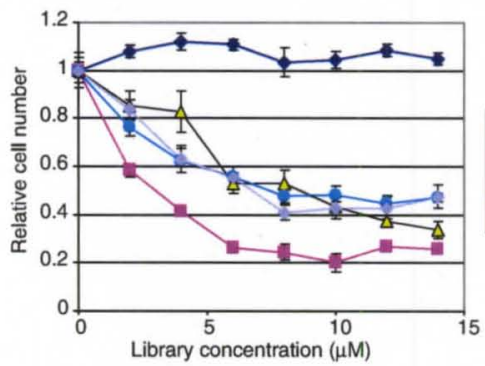

C $\quad$ A549

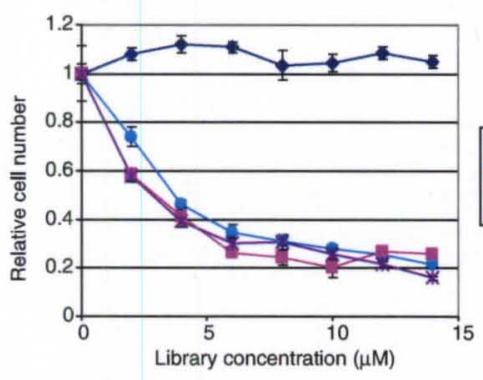

$\rightarrow-N 26 m e r$

- -GT 26mer

*-GT 34mer

\section{$\rightarrow$ G-rich}

- N 26mer
$\Delta-$ GT 10mer
- GT 26mer
$\leftarrow$ GT 0.33
$\leftarrow$ GT 0.67

E GC library

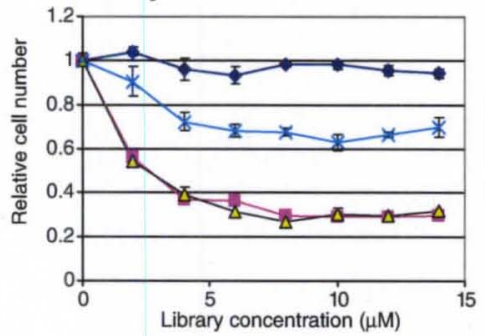

$-\mathrm{Hs} 27$

- -A549

$\triangle-$ DU145

- MCF7

Figure 3. Antiproliferative activity of libraries and oligomers. (A) Tabulation of percent decrease in cell number at $6 \mu \mathrm{M}$ (strand concentration), calculated taking untreated wells to be $100 \%$. Dark-gray shading indicates $>50 \%$ decrease; light-gray shading indicates between $30 \%$ and $50 \%$ decrease. (B) Growth curves showing the effects of all libraries, non-G-rich and G-rich, on non-cancerous fibroblasts (Hs27). Relative cell numbers at each concentration are compared to no treatment. (C) Growth curves showing the effects of GT libraries of different lengths on A549 lung cancer cells. The N library is shown for comparison. (D) Growth curves showing the effects of various GT libraries on A549 lung cancer cells. The N library is shown for comparison. (E) Growth curves showing the effects of the GC 26-mer library on four cell lines.

that although the MCF10A cells were slightly more sensitive to antiproliferative effects than the $\mathrm{Hs} 27$ skin fibroblasts, they were markedly less sensitive than the cancer cell lines, including MCF7 breast cancer cells (Supplementary Figure S1).

\section{Libraries with antiproliferative activity share features of CD spectra}

CD of DNA arises from the helical arrangement of the bases and therefore conformational characteristics (e.g. A-, B- or Z-forms of duplexes, and C- or 
G-quadruplexes) can often be inferred from CD spectra, as described in an excellent recent review article (18). In our experiments, we observed that the CD spectra of active libraries typically displayed a negative peak at $\sim 240 \mathrm{~nm}$, a strong positive peak at $260-265 \mathrm{~nm}$ and an additional maximum or shoulder (depending on the cation present) in the region $285-290 \mathrm{~nm}$ (Figure 4A). In addition, the spectra of the active libraries changed significantly between different salt solutions, with the largest peaks seen in the $100 \mathrm{mM}$ potassium chloride solution. While CD data alone cannot be used to absolutely assign structure, it is widely accepted that positive peaks close to $260 \mathrm{~nm}$ or $290 \mathrm{~nm}$ are associated with G-quadruplex formation $(18,19)$. Similarly, the conformational polymorphism suggested by the differences in CD spectra in the presence of various cations is typical of G-quadruplexes. Obviously, the shape of the spectrum for an oligonucleotide library is derived from the combined $\mathrm{CD}$ spectra from millions of sequences. Thus, the characteristic spectrum of the active GT libraries (Figure 4A) presumably represents contributions from molecules with various arrangements of G-quartets (maxima at 260 and $290 \mathrm{~nm}$ ) and non-quadruplex single-stranded molecules (maximum at $275-280 \mathrm{~nm}$ ). Somewhat surprisingly, the spectrum of the GC library (Figure 4A) was quite similar to the GT libraries and shared features suggestive of G-quadruplex formation, although the presence of duplexes or hairpins in the GC-library cannot be excluded.

Most of the inactive libraries showed a negative peak at $\sim 250 \mathrm{~nm}$ as well as a single positive peak at $\sim 275 \mathrm{~nm}$ (Figure 4B). This is typical of both ordered singlestranded and double-stranded DNA (18). The spectra of the inactive libraries changed only minimally with
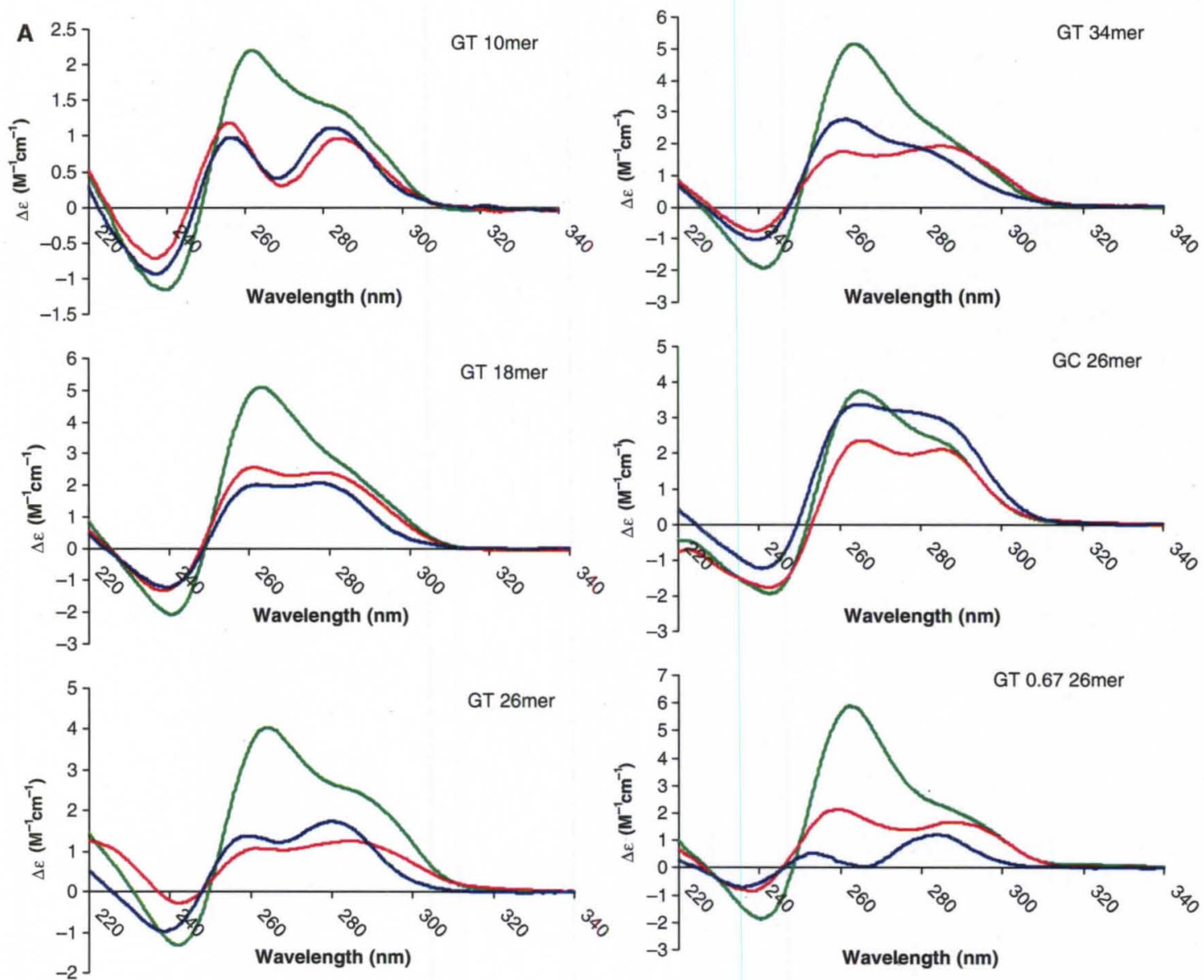

Figure 4. CD studies. Samples were prepared at a concentration of $5 \mu \mathrm{M}$ in three different solutions: water (blue), $100 \mathrm{mM} \mathrm{NaCl}$ (red) and $100 \mathrm{mM}$ $\mathrm{KCl}$ (green). (A) CD spectra of various active libraries showing a similar profile (a positive peak at $260 \mathrm{~nm}$ and a second positive peak or shoulder at $290 \mathrm{~nm}$ ), which is characteristic of G-quadruplex formation. (B) CD spectra of inactive libraries showing a similar profile (positive peak in 270-280-nm region), characteristic of non-G-quadruplex oligonucleotides. (C) Libraries with anomalous CD. The inactive GA 26-mer library CD is different from both active and inactive profiles. The GT 0.33 26-mer library has some antiproliferative activity, but its CD spectrum is similar to that of the inactive libraries. Results are shown as molar CD $(\Delta \varepsilon)$, referring to per mole of nucleotide. 

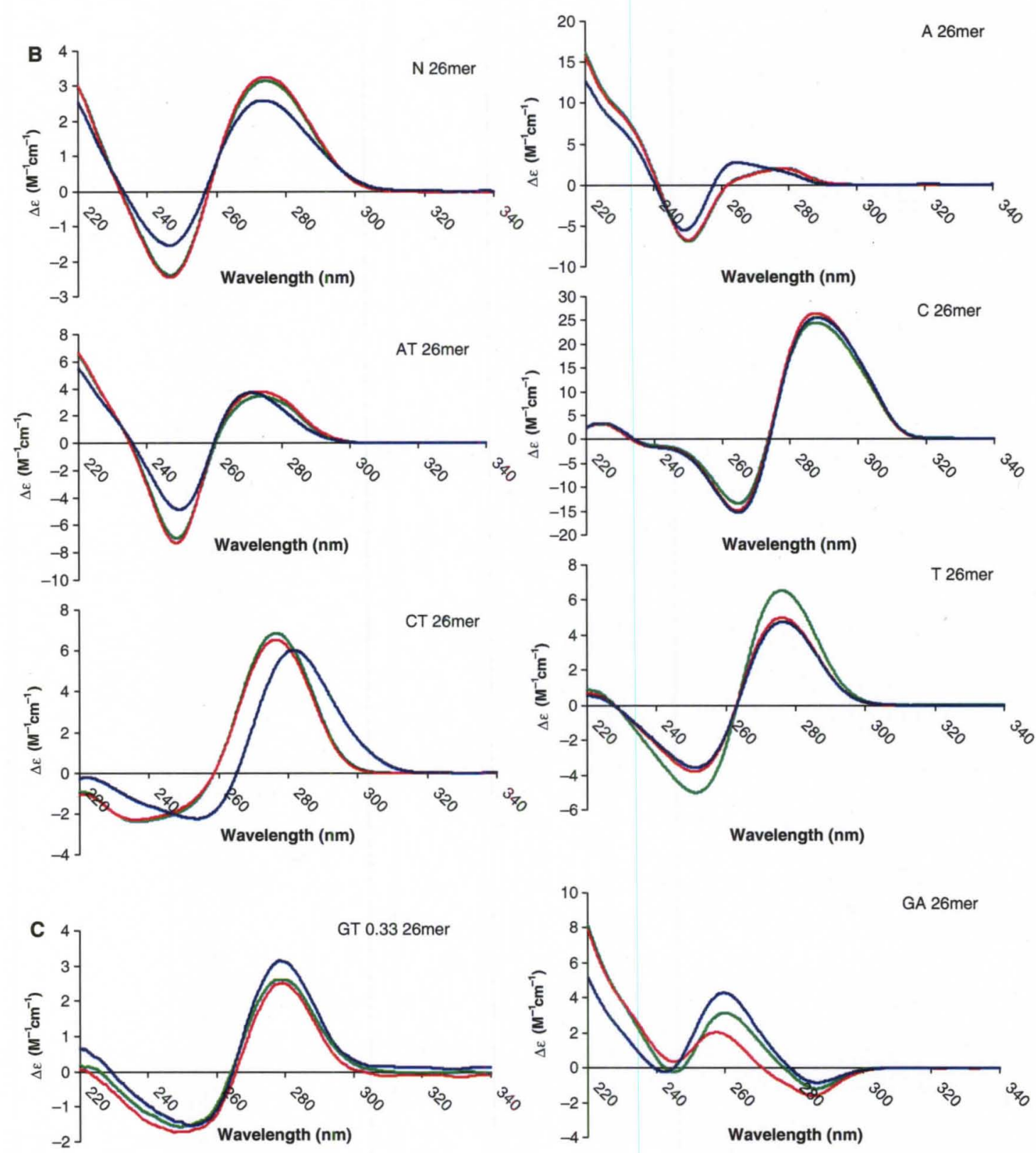

Figure 4. Continued.

different salt solutions, except for the CT library and $(\mathrm{dA})_{26}$, which showed slightly different spectral patterns in water compared to the salt-containing solutions. Interestingly, the $(\mathrm{dC})_{26}$ oligomer displayed a very large ellipticity and the peaks were slightly phase-shifted from the remaining inactive libraries, with a negative peak at $265 \mathrm{~nm}$ and positive at $285 \mathrm{~nm}$. This subtle shift in CD spectra may be due to the formation of secondary structures called i-motifs that have been reported in cytosine-rich molecules $(20,21)$, albeit usually only under slightly acidic conditions. Our theory was supported by additional experiments in buffered solutions, which showed that the amplitude and shape of the CD spectrum for $(\mathrm{dC})_{26}$ was strongly $\mathrm{pH}$-dependent and was 
typical of the i-motif $(18,21)$ at $\mathrm{pH} 5,6$ or 7 , but there was a transition to the standard single-stranded DNA spectrum at $\mathrm{pH} 8$ (Supplementary Figure S2).

The two exceptions to the general trends were the GT 0.33 26-mer library and the GA 26-mer library (Figure 4C); although the GT 0.33 26-mer library is included in the active libraries, its spectrum resembled the inactive libraries, consistent with the expected much smaller proportion of quadruplex-forming sequences and its lower level of antiproliferative activity. The spectrum of the inactive GA 26-mer library varied considerably between water, $\mathrm{NaCl}$ and $\mathrm{KCl}$, but was distinct from any of the other libraries examined, being close to zero at $240 \mathrm{~nm}$, with a positive peak at $260 \mathrm{~nm}$ and a negative peak at $285 \mathrm{~nm}$. This has been reported to be typical of $\mathrm{G}+\mathrm{A}$ containing oligonucleotides and associated with stacked guanosines, although it is not clear if this reflects the presence of quadruplexes or other ordered structures, such as alternatively base-paired duplexes (18).

Libraries with antiproliferative activity are significantly more stable in serum than inactive libraries

To assess nuclease resistance, oligonucleotides and libraries were radiolabeled at the $5^{\prime}$-end and incubated in complete medium (which contains serum-derived exonucleases) for various times. The primary purpose of this experiment was to determine nuclease stability under the exact conditions used in the MTT assays. This was considered essential, because if an oligonucleotide is degraded in the cell culture medium, it is unlikely to be active whatever other properties it has (unless the active component is derived from degradation of the oligonucleotide into nucleotides). The results (Figure 5A) showed that libraries that did not contain any $\mathrm{G}$ bases, as well as all of the monobasic oligomers tested, were highly susceptible to degradation in serum-containing medium, with most being fully degraded within $90 \mathrm{~min}$. Libraries containing guanines in any percentage had at least some molecules still intact at $72 \mathrm{~h}$, regardless of antiproliferative activity.

Although all active libraries displayed stability in the presence of serum nucleases, the degree of stability did not correlate with antiproliferative activity. For example, the moderately active GT 10-mer library was markedly less stable than the inactive N 26-mer library. However, although band density did not correspond to antiproliferative activity for the individual libraries, the mean band density of the active libraries at 24 and $72 \mathrm{~h}$ was significantly greater ( $P=0.026$ using Students' two-tailed $t$-test) than the inactive libraries (Figure 5B).

\section{Active libraries had increased cellular uptake compared to inactive libraries as shown by flow cytometry}

For this assay, four representative 26-mer libraries (the active GT and GC libraries, and the inactive $\mathrm{N}$ and GA libraries) were synthesized with a fluorophore at the 3 '-end and used to assess cellular uptake by flow cytometry. Cells were washed and harvested with trypsin prior to analysis to remove any surface-bound oligonucleotides. The relative level of uptake in a given

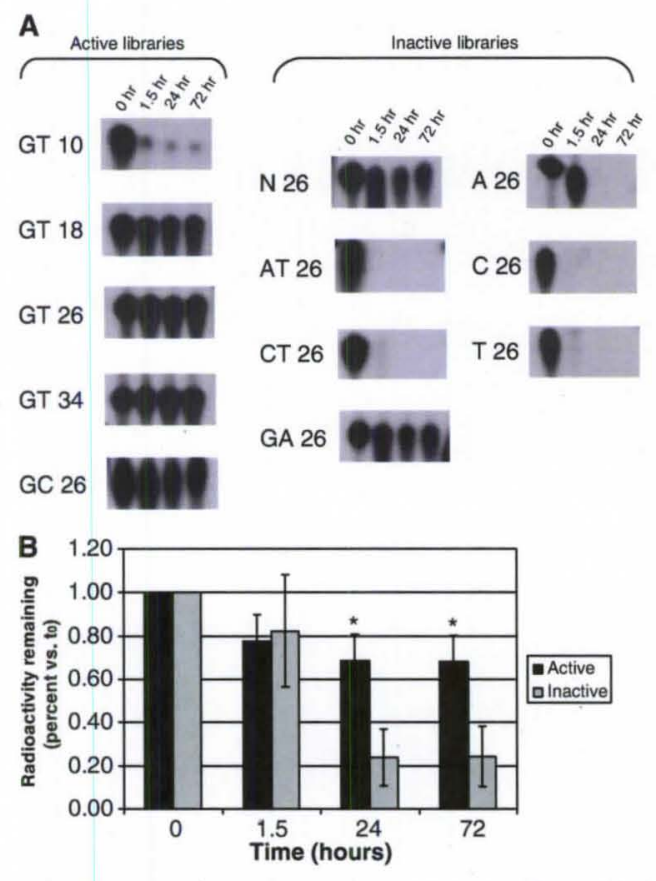

Figure 5. Nuclease-resistance in serum-containing medium. (A) Autoradiographs showing electophoretic migration of radiolabeled oligomers or libraries following incubation in complete cell culture medium, which contains serum exonucleases. (B) Mean stability of active versus inactive libraries. The difference in band density between active and inactive libraries was statistically significant at 24 and $72 \mathrm{~h}$ $\left({ }^{*} P<0.05\right.$ using Student's two-tailed $t$-test).

cell line can be estimated by determining the percent of cells that are 'positive' for the fluorophore (i.e. have a greater fluorescent intensity than the background signal, which arises from autofluorescence of the cells) after excluding non-viable cells. This type of analysis is especially useful for comparing the uptake of various libraries and oligonucleotides in a particular cell line (22), although its use for comparing uptake between different cell lines is somewhat complicated by differing levels of baseline autofluorescence between cell lines.

We observed that the active libraries, GT and GC, consistently yielded a higher proportion of fluorescent cells compared to inactive libraries in all cell lines (Figure 6). In general, the inactive $\mathrm{N}$ library had the lowest uptake of any library examined, and the inactive GA library had a similar or slightly higher uptake than the $\mathrm{N}$ library, but substantially less than the active libraries. For the GC 26-mer library, whereas its uptake in Hs27, DU145 and A549 cells was close to that of the GT library (albeit a different distribution), its uptake in MCF7 cells was considerably less than the GT library (Figure 6D). This result is noteworthy because the GC library also has reduced antiproliferative activity in this particular cell line (Figure 3E). Thus, these data suggest a correlation between relative uptake and antiproliferative activity, although with the caveat that, as mentioned above, this 


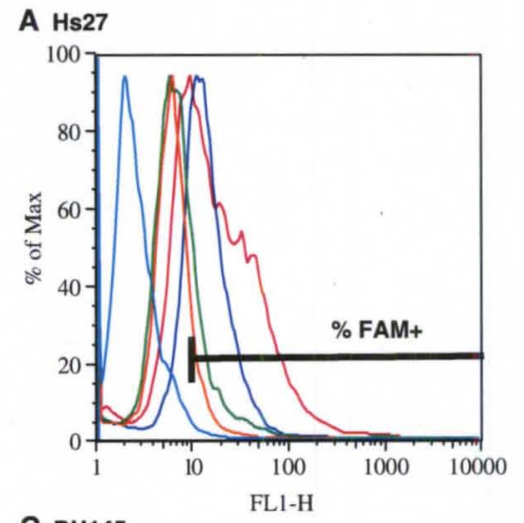

\section{B A549}

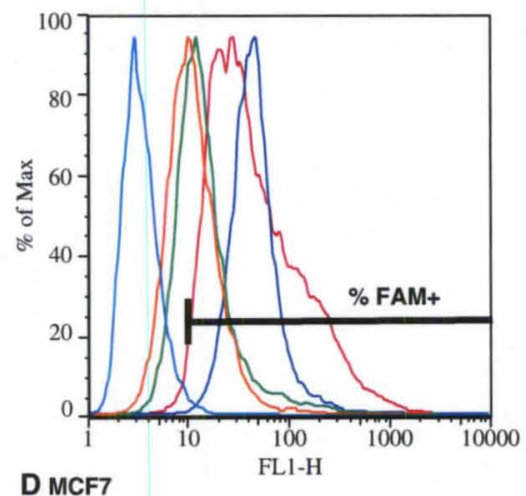

\section{DU145}

D MCF7
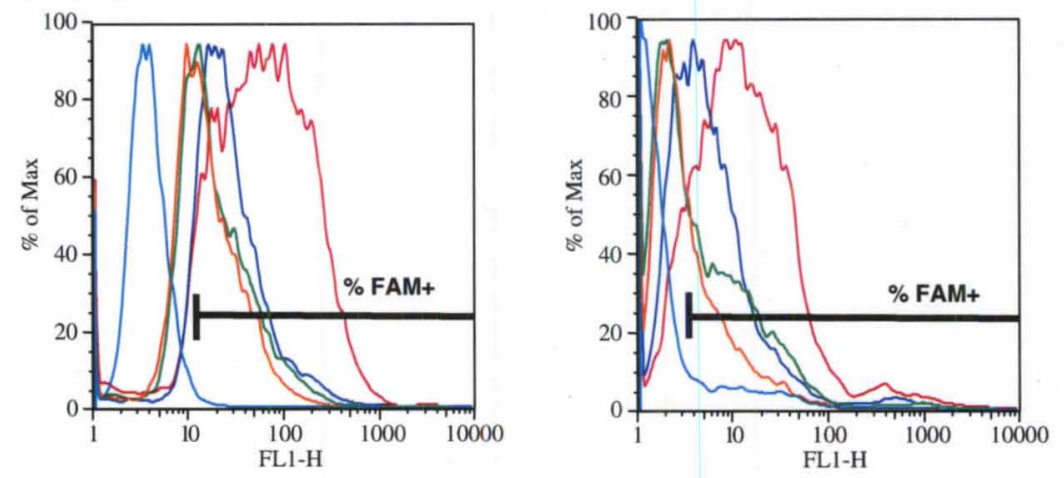

\begin{tabular}{|c|c|c|c|c|c|c|}
\hline E & \multicolumn{5}{|c|}{$\%$ FAM+ cells } & \multirow{6}{*}{$\begin{array}{l}\text { 日 Untreated } \\
\text { 日 N } \\
\text { 日 GA } \\
\text { 日 GC } \\
\text { 日 }\end{array}$} \\
\hline Cells & $\begin{array}{c}\text { None } \\
\text { background }\end{array}$ & $\mathrm{N}$ & GA & GC & GT & \\
\hline Hs27 & 1 & 10 & 20 & 64 & 61 & \\
\hline A549 & 1 & 54 & 70 & 100 & 98 & \\
\hline DU145 & 1 & 49 & 57 & 84 & 86 & \\
\hline MCF7 & 8 & 23 & 37 & 46 & 73 & \\
\hline
\end{tabular}

Figure 6. Cellular uptake of libraries and oligomers. Fluorescently labeled oligonucleotides ( $5 \mu \mathrm{M}$ strand concentration) were incubated with cells for $1 \mathrm{~h}$. Cells were harvested and stripped of surface-bound DNA using trypsin, then analyzed by flow cytometry to determine uptake. Uptake was quantified by determining the percentage of cells included in the black gate (positive for FAM after controlling for autofluorescence). (A) Uptake in Hs27 non-malignant fibroblasts. (B) Uptake in A549 cancer cells. (C) Uptake in DU145 cancer cells. (D) Uptake in MCF7 cancer cells. (E) Table of percent of FAM-positive cells for each sample.

technique may not be optimal for comparing uptake between different cell lines. We noted the non-uniform shape of the curve for the GT library compared to other libraries, which may indicate a non-uniform uptake of the GT library (some cells take up much more oligomer than others), but the reason for this is unclear at present.

\section{Active libraries and inactive libraries had quantitative} and qualitative differences in protein binding

The protein binding patterns of $5^{\prime}$-radiolabeled libraries or oligonucleotides following incubation with cell extracts were examined by electrophoretic mobility shift assay
(EMSA) and are shown in Figure 7. To start, we first analyzed the libraries and oligomers in the absence of proteins. The results (Figure 7A) confirmed the labeling and integrity of each sample and showed a quite marked dependence of electrophoretic migration on base composition, as expected. A very general observation from the EMSA experiments was that inactive libraries and oligomers displayed fewer and less intense shifted bands, when compared to the active libraries across all samples. Several shifted bands of interest were noted and are marked in Figure 7B-F. Appearance of the band marked 'Bl' was associated for the most part with nuclease-resistance libraries, but not nuclease-susceptible 

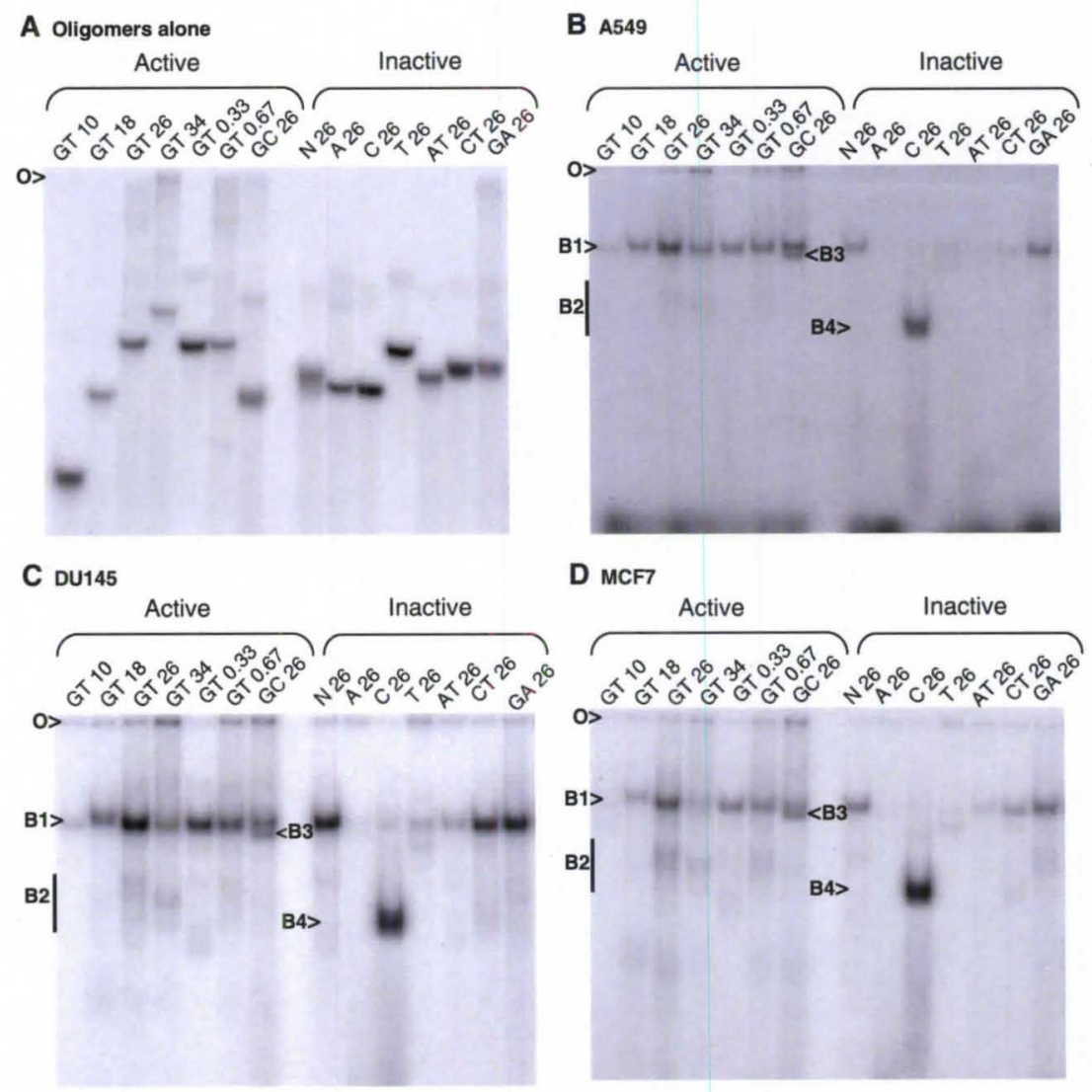

D MCF7

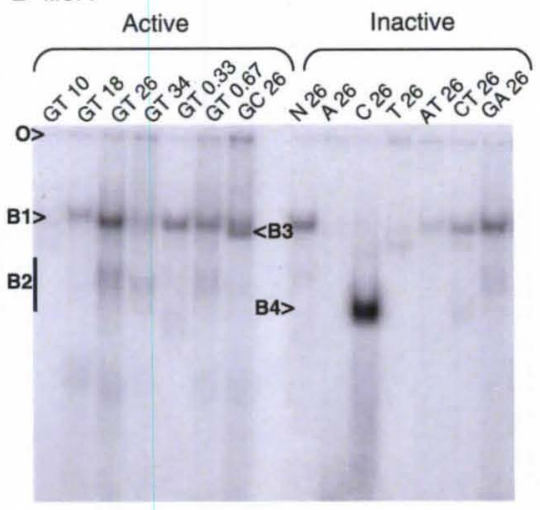

\section{E Hs27}

F Key oligomers
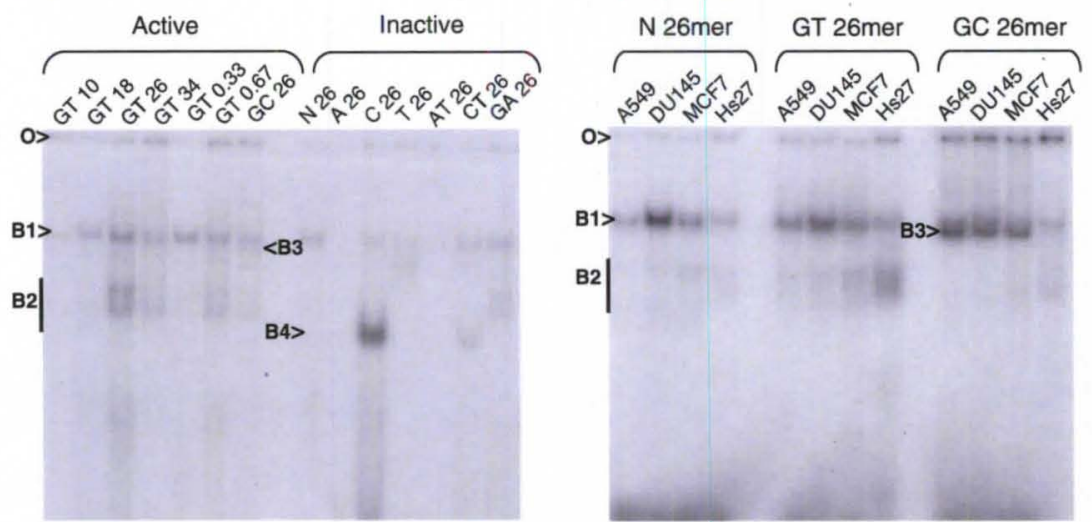

Figure 7. Protein binding of libraries and oligomers. Radiolabeled oligonucleotides were incubated with whole-cell extracts and analyzed by native gel EMSAs. Oligonucleotides were with: (A) no protein extracts (oligomers alone); (B) A549 extracts; (C) DU145 extracts; (D) MCF7 extracts; (E) Hs27 extracts; or (F) representative libraries were directly compared for binding to extracts $(5 \mu \mathrm{g})$ from the four cell types. The arrows indicate DNA-protein complexes of particular interest (see text for discussion), and ' $O$ ' indicates the origin of the gel.

libraries or oligomers (Figure 5), suggesting it may be a general DNA-binding protein. However, this was usually most intense for the most active libraries and was present at much higher levels in the cancer cell lines compared to the non-cancer cells (Figure 7F), suggesting it may contribute to the antiproliferative effects. The series of faster migrating bands indicated by 'B2' were much less intense than $\mathrm{B1}$, but are of interest because they were present 
predominantly for the most active libraries (GT26 and GT34). However, these bands were observed for Hs27 cell extracts, as well as for cancer cells (Figure 7F). The band marked ' $\mathrm{B} 3$ ' is particularly interesting because it is specific for the GC-library, which has strong antiproliferative activity, and it is present at high levels in all of the cancer cells, but is undetectable in the non-malignant cells (Figure $7 \mathbf{F}$ ). The fastest migrating band we have highlighted, 'B4', is present only in the $\mathrm{C} 26$ sample. Although this sequence has no antiproliferative activity, this band is interesting because oligo-dC sequences may form unusual structures, involving the i-motif, and very few proteins that bind specifically to such structures have been previously reported (20). We plan to follow up these studies in the future by attempting to identify proteins of interest using mass spectrometry-based techniques.

Interpretation of the EMSA data in the presence of protein (Figure 7B-F) has the potential to be somewhat complicated because the presence of shifted bands may depend not only on protein binding activity, but on nuclease-resistance as well. The relative nuclease-susceptibility under these conditions was expected to reflect the results from our serum stability assay (Figure 5), but may not be exactly the same because cell extracts contain endonucleases, as well as exonucleases. To further investigate these issues, we performed nuclease-resistance assays in the presence of the DU145 cellular extracts that were used in the EMSA experiments. We examined stability after incubation for a short time $(15 \mathrm{~min})$, which mimics the EMSA conditions, and found no significant nuclease degradation (Supplementary Figure S3A). These results verify that the absence of a shifted band in the EMSA is due to the complex not being formed, rather than the oligonucleotides being degraded by nucleases. After longer incubation $(18 \mathrm{~h})$, which we anticipate will reflect the persistence of oligonucleotides inside cells, we observed (Supplementary Figure S3B) relative stabilities similar to those reported above for long-term stability in cell culture medium (Figure 5). This confirms our conclusions regarding the relationship between nuclease resistance, protein binding and antiproliferative activity (i.e. that, while active libraries are invariably stable and display strong protein binding, not all libraries that are stable and display strong protein binding are active). Furthermore, the data also suggest that protein binding may be responsible for nuclease resistance and not vice versa.

\section{CONCLUSIONS}

In this study, we have examined the biological properties and $\mathrm{CD}$ spectra of oligonucleotide libraries. The purpose of this exercise was primarily to test our hypothesis that G-rich libraries would be enriched in quadruplexes, which might make them useful starting points for the development of new therapeutic aptamers using SELEX or similar technologies.

The results of our analyses seem to confirm that G-rich oligodeoxynucleotide libraries do indeed contain large numbers of quadruplex-forming sequences, and they are more nuclease-resistant and efficiently internalized by cells compared to the standard ' $N$ library' currently used in SELEX. Experiments are now underway to determine if G-rich libraries can be used for SELEX and whether they will allow faster evolution of aptamers for in vivo use.

While many of the observed properties of G-rich libraries were anticipated, the strong and cancer-selective growth inhibitory effects of some GT and GC libraries were unforeseen. When analyzing a library of molecules, it is important to remember that the results are the averages of the effects of all the different molecules within the library. Given our previous work, showing that several different quadruplex-forming oligonucleotides have cancer-selective antiproliferative activity $(19,23)$, we expected that G-rich libraries might contain a small proportion of active molecules. However, while we anticipated that a G-rich library might contain some very potent molecules, we presumed that the vast majority of the estimated 67000000 sequences in such a library would be ineffective, such that the library as a whole would display at best a modest growth inhibitory effect against cancer cells. Thus, the finding that certain GT and GC libraries have very potent and tumor-selective antiproliferative effects against a variety of cancer cell types was somewhat surprising. Remarkably, the antiproliferative activity of these libraries was comparable to some individual G-rich oligodeoxynucleotides that are currently in pre-clinical development or clinical trials (11).

Although a full understanding of the antiproliferative properties of active libraries will require further efforts, the work that we have described provides some clues to the mechanism of action for these effects. The restriction of the activity to only certain GT and GC libraries indicates that it cannot be a non-specific effect of phosphodiester oligonucleotides or simply due to the presence of deoxyguanosine nucleotides (either in the context of an oligonucleotide or as nucleobases resulting from oligomer degradation), because some dG-containing libraries, including the $\mathrm{GA}$ and $\mathrm{N}$ libraries, had no activity. In addition, although active libraries are generally more stable than inactive libraries, nuclease-resistance alone cannot explain the antiproliferative effects because some of the inactive libraries displayed comparable stability.

In view of our observations, we now propose that antiproliferative activity requires all of the following: nuclease-resistance, efficient cellular uptake and binding to a specific protein (or proteins). It is not yet clear if quadruplex formation, per se, is necessary for activity, or whether it is simply that quadruplex-forming oligonucleotides generally have the aforementioned properties. One possible candidate for the complex in the EMSAs that appears to be most relevant for activity (band ' $\mathrm{Bl}$ ' in Figure 7) is nucleolin. This protein is highly expressed in cancer cells and is a general DNA binding protein, but binds particularly strongly to a variety of quadruplex-forming sequences (24-27). It is also the molecular target of an existing G/T oligonucleotide aptamer (11). However, further studies will be required to verify this possibility and to reconcile why some 
libraries bind to this protein (if it is indeed band B1), yet do not have enhanced cellular uptake or antiproliferative activity. Interestingly, the GC library appears to have a different cancer cell selectivity compared to the GT libraries and forms a specific protein complex (band 'B3' in Figure 7), suggesting that its mechanism of action and molecular target may be distinct from the GT oligonucleotides.

In summary, the potent intrinsic and selective antiproliferative activity of the GT and GC libraries is an unexpected and wholly novel finding. Our results indicate that cancer-selective antiproliferative activity is a common characteristic of G-rich DNA oligonucleotides, which may explain why numerous different G-rich oligonucleotides have been reported as potential anticancer agents (11). Although many different mechanisms of action have been proposed for these, our results suggest there may be common mechanisms (related to nuclease resistance, protein binding and cellular uptake) that contribute to the activity of G-rich oligonucleotides. Further research is warranted to elucidate the mechanism of action for these cancer-selective effects, as this may lead to insights into basic cancer cell biology, as well as to the identification of novel molecular targets and new oligomer-based therapies for cancer.

\section{SUPPLEMENTARY DATA}

Supplementary Data are available at NAR Online.

\section{ACKNOWLEDGEMENTS}

The authors wish to thank Magdalena Dailey, M.D. and Kara Sedoris, Ph.D. of the James Graham Brown Cancer Center at the University of Louisville for assistance with the CD data. They also thank Andrew N. Lane, J. Brad Chaires and John O. Trent for their constructive criticisms of the draft manuscript.

\section{FUNDING}

The National Institutes of Health (R01CA122383 to P.B.); the Department of Defense Prostate Cancer Research Program (fellowship PC073700 to E.C.); the Kentucky Lung Cancer Research Program (to P.B.); the Institute for Molecular Diversity and Drug Design (fellowship to E.C.). Funding for open access charge: Department of Defense [Prostate Cancer Research Program training grant (to E.C. and P.B.)].

Conflict of interest statement. P.B. has a financial interest in Antisoma, PLC (London, England) as a shareholder, consultant, and recipient of research support. P.B. has a financial interest in Transmed Oncology, Inc. as a shareholder, consultant, and recipient of research support.

\section{REFERENCES}

1. Mayer,G. (2009) The chemical biology of aptamers. Angew. Chem. Int. Ed. Engl., 48, 2672-2689.
2. Ireson,C.R. and Kelland,L.R. (2006) Discovery and development of anticancer aptamers. Mol. Cancer Ther., 5, 2957-2962.

3. Pestourie,C., Tavitian,B. and Duconge,F. (2005) Aptamers against extracellular targets for in vivo applications. Biochimie, 87, 921-930.

4. Nimjee,S.M., Rusconi,C.P. and Sullenger,B.A. (2005)

Aptamers: an emerging class of therapeutics. Annu. Rev. Med., 56, 555-583.

5. Ng,E.W., Shima,D.T., Calias,P., Cunningham,E.T. Jr, Guyer,D.R. and Adamis,A.P. (2006) Pegaptanib, a targeted anti-VEGF aptamer for ocular vascular disease. Nat. Rev. Drug Discov., 5, 123-132.

6. Gragoudas,E.S., Adamis,A.P., Cunningham,E.T. Jr, Feinsod,M. and Guyer,D.R. (2004) VEGF Inhibition Study in Ocular Neovascularization Clinical Trial Group. Pegaptanib for neovascular age-related macular degeneration. N. Engl. J. Med., 351, 2805-2816.

7. Lee,S.W. and Sullenger,B.A. (1997) Isolation of a nuclease-resistant decoy RNA that can protect human acetylcholine receptors from myasthenic antibodies. $N a t$. Biotechnol., 15, 41-45.

8. Bock,L.C., Griffin,L.C., Latham,J.A., Vermaas,E.H. and Toole,J.J. (1992) Selection of single-stranded DNA molecules that bind and inhibit human thrombin. Nature, 355, 564-566.

9. Oney,S., Nimjee,S.M., Layzer,J., Que-Gewirth,N., Ginsburg,D., Becker,R.C., Arepally,G. and Sullenger,B.A. (2007) Antidote-controlled platelet inhibition targeting von Willebrand factor with aptamers. Oligonucleotides, 17, 265-274.

10. Weiss,S., Proske,D., Neumann,M., Groschup,M.H., Kretzschmar,H.A., Famulok,M. and Winnacker,E.L. (1997) RNA aptamers specifically interact with the prion protein $\operatorname{PrP} . J$. Virol., 71, 8790-8797.

11. Bates,P.J., Laber,D.A., Miller,D.M., Thomas,S.D. and Trent,J.O. (2009) Discovery and development of the G-rich oligonucleotide AS1411 as a novel treatment for cancer. Exp. Mol. Pathol., 86, 151-164.

12. Tuerk,C. and Gold,L. (1990) Systematic evolution of ligands by exponential enrichment: RNA ligands to bacteriophage T4 DNA polymerase. Science, 249, 505-510.

13. Ellington,A.D. and Szostak,J.W. (1990) In vitro selection of RNA molecules that bind specific ligands. Nature, 346, 818-822.

14. Phillips,J.A., Lopez-Colon,D., Zhu,Z., Xu,Y. and Tan,W. (2008) Applications of aptamers in cancer cell biology. Anal. Chim. Acta, 621, 101-108.

15. Keefe,A.D. and Cload,S.T. (2008) SELEX with modified nucleotides. Curr. Opin. Chem. Biol., 12, 448-456.

16. Girvan,A.C., Teng,Y., Casson,L.K., Thomas,S.D., Jüliger,S., Ball,M.W., Klein,J.B., Pierce,W.M., Barve,S.S. and Bates,P.J. (2006) AGRO100 inhibits activation of nuclear factor-kappaB (NF-kappaB) by forming a complex with NF-kappaB essential modulator (NEMO) and nucleolin. Mol. Cancer Ther., 5, $1790-1799$.

17. Shamovsky,I., Ivannikov,M., Kandel,E.S., Gershon,D. and Nudler,E. (2006) RNA-mediated response to heat shock in mammalian cells. Nature, 440, 556-560.

18. Kypr J., Kejnovská,I., Renciuk,D. and Vorlícková,M. (2009) Circular dichroism and conformational polymorphism of DNA. Nucleic Acids Res., 37, 1713-1725.

19. Dapić,V., Abdomerović,V., Marrington,R., Peberdy,J., Rodger,A., Trent,J.O. and Bates,P.J. (2003) Biophysical and biological properties of quadruplex oligodeoxyribonucleotides. Nucleic Acids Res., 31, 2097-2107.

20. Guéron,M. and Leroy,J.L. (2000) The i-motif in nucleic acids. Curr. Opin. Struct. Biol., 10, 326-331.

21. Guo,K., Gokhale, V., Hurley,L.H. and Sun,D. (2008) Intramolecularly folded G-quadruplex and i-motif structures in the proximal promoter of the vascular endothelial growth factor gene. Nucleic Acids Res., 36, 4598-4608.

22. Wu,C.C., Castro,J.E., Motta,M., Cottam,H.B., Kyburz,D. Kipps,T.J., Corr,M. and Carson,D.A. (2003) Selection of oligonucleotide aptamers with enhanced uptake and activation of human leukemia B cells. Hum. Gene Ther., 14, $849-860$. 
23. Dapić,V., Bates,P.J., Trent,J.O., Rodger,A., Thomas,S.D. and Miller,D.M. (2002) Antiproliferative activity of G-quartet-forming oligonucleotides with backbone and sugar modifications. Biochemistry, 41, 3676-3685.

24. Dempsey,L.A., Sun,H., Hanakahi,L.A. and Maizels,N. (1999) G4 DNA binding by LRI and its subunits, nucleolin and hnRNP D, A role for G-G pairing in immunoglobulin switch recombination. J. Biol. Chem., 274, 1066-1071.

25. Hanakahi,L.A., Sun,H. and Maizels,N. (1999) High affinity interactions of nucleolin with G-G-paired rDNA. J. Biol. Chem., 274, 15908-15912.
26. Dickinson,L.A. and Kohwi-Shigematsu,T. (1995) Nucleolin is a matrix attachment region DNA-binding protein that specifically recognizes a region with high base-unpairing potential. Mol. Cell. Biol., 15, 456465

27. Ishikawa,F., Matunis,M.J., Dreyfuss,G. and Cech,T.R. (1993) Nuclear proteins that bind the pre-mRNA 3' splice site sequence $\mathrm{r}(\mathrm{UUAG} / \mathrm{G})$ and the human telomeric DNA sequence d(TTAGGG)n. Mol. Cell. Biol., 13, 4301-4310. 


\section{SUPPLEMENTARY DATA: METHODS}

\section{Antiproliferative assay of oligomers on MCF10A cells}

MCF10A non-malignant mammary epithelial cells were cultured in MEBM supplemented according to ATCC guidelines (BPE $13 \mathrm{mg} / \mathrm{ml}, 2 \mathrm{ml}$; hydrocortisone 0.5 $\mathrm{mg} / \mathrm{ml}, 0.5 \mathrm{ml}$; hEGF $10 \mathrm{ug} / \mathrm{ml}, 0.5 \mathrm{ml}$; insulin $5 \mathrm{mg} / \mathrm{ml}, 0.5 \mathrm{ml}$ ). Cells were incubated at $37^{\circ} \mathrm{C}$ in $5 \% \mathrm{CO}_{2}$. For the MTT assays, 3000 cells were plated per well and assays were performed according to methods described in the manuscript.

\section{Circular dichroism at varying $\mathbf{p H}$}

The $(\mathrm{dC})_{26}$ oligomer was prepared at a concentration of $5 \mu \mathrm{M}$ (strand concentration) in 50 $\mathrm{mM}$ Tris acetate buffer that was $\mathrm{pH}$-adjusted using glacial acetic acid to final values of 5 , 6,7 , and 8. Samples were incubated and analyzed according to methods described in the manuscript.

\section{Nuclease-resistance in DU145 protein extracts}

Libraries and oligomers were end-labeled as described in the manuscript. To match the conditions of the protein-binding assay, $50,000 \mathrm{cpm}$ of oligomer was combined with 2.5 $\mu \mathrm{g}$ DU145 whole cell lysate and protein binding buffer, as described for the EMSA in the manuscript. Samples were incubated at $37^{\circ} \mathrm{C}$ for $15 \mathrm{~min}$, then electrophoresed on a $12 \%$ denaturing gel as described in the manuscript for the nuclease resistance assay. To examine nuclease resistance under conditions mimicking the cellular environment, similar experiments were carried out using $50,000 \mathrm{cpm}$ of oligomer with $2.5 \mu \mathrm{g}$ DU145 whole cell lysate, but without protein-binding buffer, incubated at $37^{\circ} \mathrm{C}$ for $18 \mathrm{~h}$.

\section{SUPPLEMENTARY DATA: FIGURE LEGENDS}

Supplementary Figure 1: Antiproliferative activities of libraries and oligomers against MCF10A cells.

(a) Growth curves showing the effects of all libraries on non-cancerous breast epithelial cells (MCF10A). Relative cell numbers at each concentration are compared to no treatment. For comparison, the following are also shown: (b) Hs27 non-cancerous fibroblasts; and (c) MCF7 breast adenocarcinoma cells.

Supplementary Figure 2: Circular dichroism (CD) of C 26-mer oligodeoxynucleotide at varying $\mathrm{pH}$. Samples containing $5 \mu \mathrm{M}$ oligomer in Tris-acetate buffer were analyzed and the results shown are molar circular dichroism $(\Delta \varepsilon)$, referring to per mole of nucleotide.

Supplementary Figure 3: Nuclease-resistance in the presence of DU145 protein extracts. 5 '-Radiolabeled libraries and oligomers were incubated with protein extracts (prepared as described for EMSA studies) at $37^{\circ} \mathrm{C}$ for the time indicated, and then electrophoresed on a $12 \%$ polyacrylamide denaturing gel. " $\mathrm{O}$ " indicates the origin of the gel. 


\section{Supplementary Figure 1 (new)}

\section{A. MCF10A}

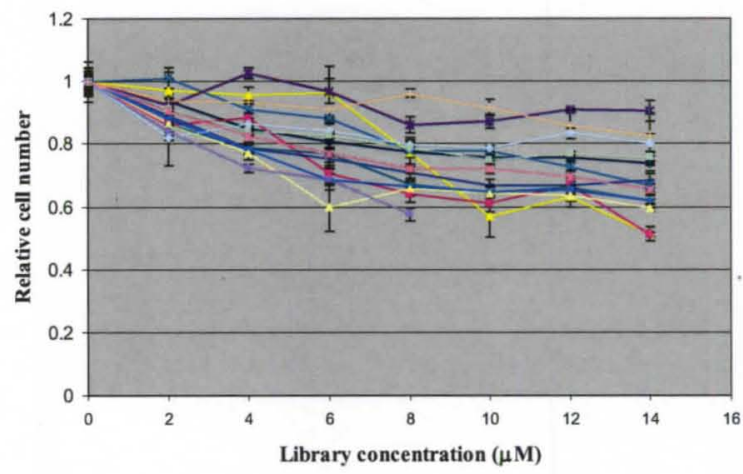

B. $\mathrm{Hs} 27$

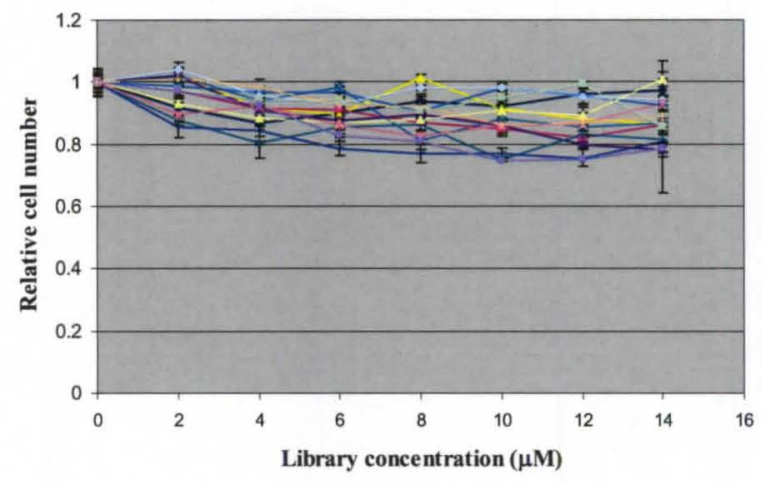

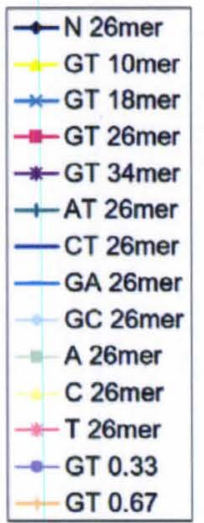

C. MCF7

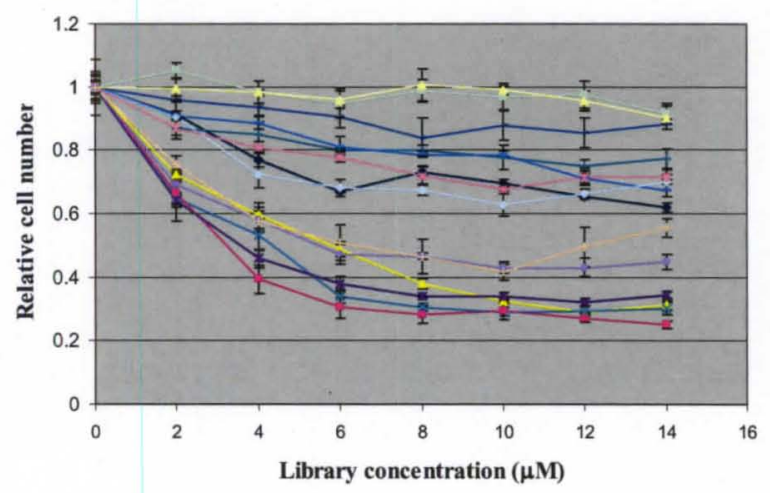




\section{Supplementary Figure 2 (new)}

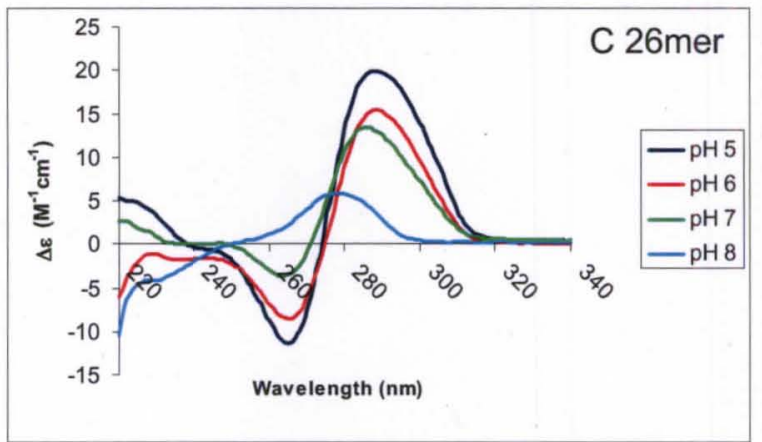




\section{Supplementary Figure 3 (new)}

\section{A. 15 minutes}

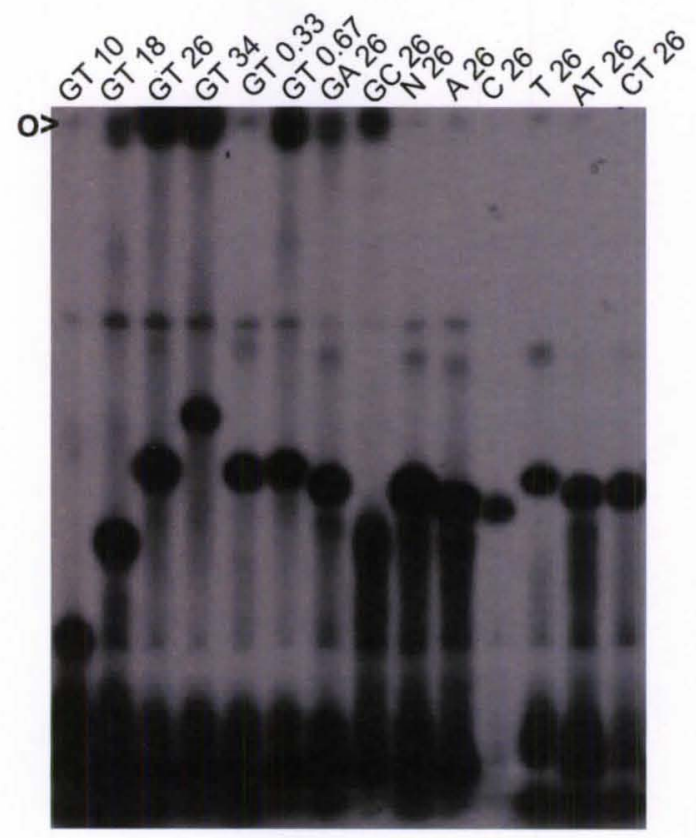

B. 18 hours

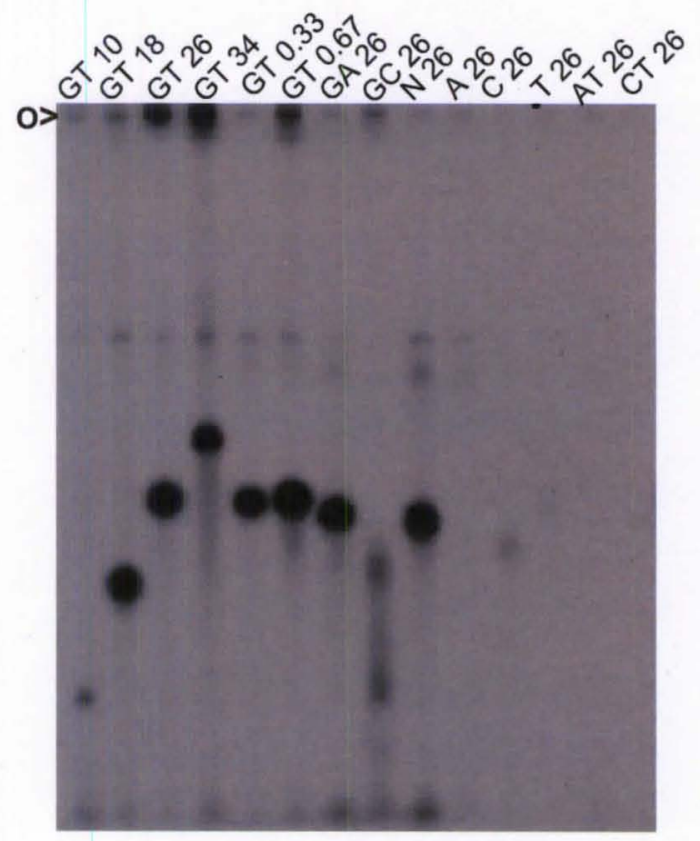




\section{APPENDIX B}

\section{SELEX sequences from $\mathrm{N}$ and GT library using PC3 prostate cancer cells}

PC3, N library

\section{Round 1:}

1) AgtAgGACtCGAgtgAgCGtgCGAgA

2) CTATTATGACAGTGCATGTTAGCCAT

3) GACGTGACTTATATAATTATGCATGG

4) ACCTATCTTCTCTAAGCTACTGAAGG

5) CTGGTACTATGGAAGAGATTGGTTCA

6) GgGGTAAGTAGATGGCAGCGTAGgCA

7) AGGAGTGGCACCAAAGTTCTGGCCTG

8) AGAAGgAAATCCGCTATCCAAAGTAC

9) AgGAAACACGgGAGATTACCTCTGCT

10) GAACCCCCGAACAGAAAAACCAGTCA

11) ACATCAAGTGCTGACGGTTGCACGAG

12) ACATCAAGTGCTGACGGTTGCACGAG

\section{Round 2:}

1) ACTCGTGAGTATCGTCGTC (GACGACGATACTCACGAG)

2) AAATAAAATCGTACGCAGCAGGGAAC $(1+1)$

3) TGGGAAATCCGATAGGCACTCAATGG

4) GACGAAGAAATGCACATGCAAAACTG

5) AACATTGGTAGACACAGTATTTTCCA

6) TAAAATGCGGAAAATAAGGGAACCGT

7) GATTGTGAAAAACAAATGACGTCGCG

8) TCGTTATGGACGGTGCGGAGGCGCAG

9) GTGTGgTtTGtgtGgGGgGtGgtGTT

10) GCACTACGTAAATCGGTGCGCGAAGC

11) ATAAAAAGAGGTGTCGGATCACGGAT

12) ATGGACTTCATGCCAAAAGCAAGTCG

13) TACGGAATAATGAGATTGTCAACGGA

14) GGTTGAGAAAAACCGATGGTCAGT

15) AGGTACATAACAATCTGACAAAGCAA

16) GGGGGATAGACAGTG

17) CGGACAAGAAATGTGTACTAGTGGTG 
18) AAAGGATCACGTAGATGGATACGCAC

19) ATGGAGGAAAACTCAGCCCCAGAT

Round 3:

1) GCTGAGATGACTCATCCTCCCAGGCACAGCAAGAAGAAACAGGCAGCATGCCCATTG CTTGTTTATTCCTTGTTTTAATTTTCATTATTACGTTTCTCAGGAAATGCACGCCTT AGCTAACAGTTACTGGCAAAAAAATAAATAAATAAATAAGGTGTTTGAGTTCTTGCC AACATTTGGGGAAGCTGAATGAGATGCCTAGAGGAGGGTTGGCCCATCCTTTGCTTA AAGTTTTCTTTCCTTTTTTCTTTTTTGTTTTTTTTCTGTCCTTTAGCCCATGAAGAG CATCATGCTTTTGAAGGTGGACGTGGAAAACACTAATTGCACTCTGTAAAGAATTCT TTGTCCTTTGCTGATTGGTTTTGGAAACGGTCTTAACAGGAGGGAGAGTGAAGAGAA GACTTGCCG $(3+21)$

2) ACTACGGAGGGGCTAGAGCAACAAGT $(6+58)$

3) GTTTTGGTTGGGGGTGGGGGTTGGGT

4) *GCTGAGATGACTCATCCTCCCAGGCACAGCAAGAAGAAACAGGCAGCATGCCCATT GCTTGTTTATTCCTTGTTTTAATTTTCATTATTACGTTTCTCAGGAAATGCACGCCT TAGCTAACAGTTACTGGCAAAAAAATAAATAAATAAATAAGGTGTTTGAGTTCTTGC CAACATTTGGGGAAGCTGAATGAGATGCCTAGAGGAGGGTTGGCCCATCCTTTGCTT AAAGTTTTCTTTCCTTTTTTTCTTTTTTGTTTTTTTTCTGTCCTTTGGCCCATGAAG AGCATCATGCTTTTGAAGGTGGACGTGGAAAACACTAATTGCACTCTGTAAAGAATT CTTTGTCCTTTGCTGATTGGTTTTGGAAACGGTCTTAACAGGAGGGAGAGTGAAGAG AAGACTTGCCG $(3+21)$

5) CGGCAAGTCTTCTCTTCACTCTCCCTCCTGTTAAGACCGTTTCCAAAACCAATCAGC AAAGGACAAAGAATTCTTTACAGAGTGCAATTAGTGTTTTCCACGTCCACCTTCAAA AGCATGATGCTCTTCATGGGCCAAAGGACAGAAAAAAAACAAAAAAGAAAAAAAGGA AAGAAAACTTTAAGCAAAGGATGGGCCAACCCTCCTCTAGGCATCTCATTCAGCTTC CCCAAATGTTGGCAAGAACTCAAACACCTTATTTATTTATTTATTTTTTTGCCAGTA ACTGTTAGCTAAGGCGTGCATTTCCTGAGAAACGTAATAATGAAAATTAAAACAAGG AATAAACAAGCAATGGGCATGCTGCCTGTTTCTTCTTGCTGTGCCTGGGAGGATGAG TCATCTCAGC $(2+18)$

6) ACTACGGAGGGGCTAGAGCAACAAGT $(6+58)$

7) GTTGCACTTCTGAATTCAACTCTCGG

8) ACTACGGAGgGGCTAGAGCAACAAGT $(6+58)$

9) ACTACGGAGGGGCTAGAGCAACAAGT $(6+58)$

10) ACTACGGAGGGGCTAGAGCAACAAGT $(6+58)$

11) GCTGAGATGACTCATCCTCCCAGGCACAGCAAGAAGAAACAGGCAGCATGCCCATTG CTTGTTTATTCCTTGTTTTAATTTTCATTATTACGTTTCTCAGAAAATGCACGCCTT AGCTAACAGTTACTGGCAAAAAAATAAATAAATAAATAAGGTGTTTGAGTTCTTGCC AACATTTGGGGAAGCTGAATGAGATGCCTAGAGGAGGGTTGGCCCATCCTTTGCTTA AAGTTTTCTTTCCTTTTTTTCTTTTTTGTTTTTTTTCTGTCCTTTAGCCCATGAAGA GCATCATGCTTTTGAAGGTGGACGCGGAAAACACTAATTGCACTCTGTAAAGAATTC TTTGTCCTTTGCTGATTGGTTTTGGAAACGGTCTTAACAGGAGGGAGAGTGAAGAGA AGACTTGCCG $(3+21)$

12) CGGCAAGTCTTCTCTTCACTCTCCCTCCTGTTAAGACCGTTTCCAAAACCAATCAGC AAAGGACAAAGAATTCTTTACAGAGTGCAATTAGTGTTTTCCACGTCCACCTTCAAA AGCATGATGCTCTTCATGGGCCAAAGGACAGAAAAAAAACAAAAAAGAAAAAAAGGA 
AAGAAAACTTTAAGCAAAGGATGGGCCAACCCTCCTCTAGGCATCTCATTCAGCTTC CCCAAATGTTGGCAAGAACTCAAACACCTTATTTATTTATTTATTTTTTTGCCAGTA ACTGTTAGCTAAGGCGTGCATTTCCTGAGAAACGTAATAATGAAAATTAAAACAAGG AATAAACAAGCAATGGGCATGCTGCCTGTTTCTTCTTGCTGTGCCTGGGAGGATGAG TCATCTCAGC $(2+18)$

13) ACTACGGAGGGGCTAGAGCAACAAGT $(6+58)$

14) TGTTGTGGTGGGGGGGGGGTTGTTTT

\section{Round 4:}

1) ACTACGGAGGGGCTAGAGCAACAAGT $(23+41)$

2) GCTGAGATGACTCATCCTCCCAGGCACAGCAAGAAGAAACAGGCAGCATGCCCATTG CTTGTTTATTCCTTGTTTTAATTTTCATTATTACATTTCTCAGGAAATGCACGCCTT AGCTAACAGTTACTGGCAAAAAAATAAATAAATAAATAAGGTGTTTGAGTTCTTGCC AACATTTGGGGAAGCTGAATGAGATGCCTAGAGGAGGGTTGGCCCATCCTTTGCTTA AAGTTTTCTTTCCTTTTTTTCTTTTTTGTTTTTTTTCTGTCCTTTGGCCCATGAAGA GCATCATGCTTTTGAAGGTGGACGTGGAAAACACTAATTGCACTCTGTAAAGAATTC TTTGTCCTTTGCTGATTGGTTTTGGAAACGGTCTTAACAGGAGGGAGAGTGAAGAGA AGACTTGCCG $(1+23)$

3) ACTACGGAGGGGCTAGAGCAACAAGT $(23+41)$

4) ACTACGGAGGGGCTAGAGCAACAAGT $(23+41)$

5) ACTACGGAGGGGCTAGAGCAACAAGT $(23+41)$

6) ACTACGGAGGGGCTAGAGCAACAAGT $(23+41)$

7) ACTACGGAGGGGCTAGAGCAACAAGT $(23+41)$

8) ACTACGGAGGGGCTAGAGCAACAAGT $(23+41)$

9) ACTACGGAGGGGCTAGAGCAACAAGT $(23+41)$

10) ACTACGGAGGGGCTAGAGCAACAAGT $(23+41)$

11) ACTACGGAGGGGCTAGAGCAACAAGT $(23+41)$

12) ACTACGGAGGGGCTAGAGCAACAAGT $(23+41)$

13) ACTACGGAGGGGCTAGAGCAACAAGT $(23+41)$

14) ACTACGGAGGGGCTAGAGCAACAAGT $(23+41)$

15) ACTACGGAGGGGCTAGAGCAACAAGT $(23+41)$

16) ACTACGGAGGGGCTAGAGCAACAAGT $(23+41)$

17) ACTACGGAGGGGCTAGAGCAACAAGT $(23+41)$

18) ACTACGGAGGGGCTAGTGCAACAAGT

19) ACTACGGAGGGGCTAGAGCAACAAGT $(23+41)$

20) ACTACGGAGGGGCTAGAGCAACAAGT $(23+41)$

21) ACTACGGAGGGGCTAGAGCAACAAGT $(23+41)$

22) ACTACGGAGGGGCTAGAGCAACAAGT $(23+41)$

23) ACTACGGAGGGGCTAGAGCAACAAGT $(23+41)$

24) ACTACGGAGGGGCTAGAGCAACAAGT $(23+41)$

25) ACTACGGAGGGGCTAGAGCAACAAGT $(23+41)$

Round 5:

1) ACTACGGAGgGGCTAGAGCAACAAGT (35+29)

2) ACTACGGAGgGGCTAGAGCAACAAGT $(35+29)$ 
3) GCTGAGATGACTCATCCTCCCAGGCACAGCAAGAAGAAACAGGCAGCATGCCCATTG CTTGTTTATTCCTTGTTTTAATTTTCATTATTACGTTTCTCAGGAAATGCACGCCTT AGCTAACAGTTACTGGCAAAAAAATAAATAAATAAATAAGGTGTTTGAGTTCTTGCC AACATTTGGGGAAGCTGAATGAGATGCCTAGAGGAGGGTTGGCCCATCCTTTGCTTA AAGTTTTCTTTCCTTTTTTTCTTTTTTGTTTTTTTTCTGTCCTTTAGCCCATGAAGA GCATCATGCTTTTGAAGGTGGACGTGGAAAACACTAATTGCACTCTGTAAAGAATTC TTTGTCCTTTGCTGATTGGTTTTGGAAACGGTCCTAACAGGAGGGAGAGTGAAGAGA AGACTTGCCG $(11+13)$

4) GCTGAGATGACTCATCCTCCCAGGCACAGCAAGAAGAAACAGGCAGCATGCCCATTG CTTGTTTATTCCTTGTTTTAATTTTCATTATTACGTTTCTCAGGAAATGCACGCCTT AGCTAACAGTTACTGGCAAAAAAATAAATAAATAAATAAGGTGTTTGAGTTCTTGCC AACATTTGGGGAAGCTGAATGAGATGCCTAGAGGAGGGTTGGCCCATCCTTTGCTTA AAGTTTTCTTTCCTTTTTTTCTTTTTTGTTTTTTTTCTGTCCTTTAGCCCATGAAGA GCATCATGCTTTTGAAGGTGGACGTGGAAAACACTAATTGCACTCTGTAAAGAATTC TTTGTCCTTTGCTGATTGGTTTTGGAAACGGTCTTAACAGGAGGGAGAGTGAAGAGA AGACTTGCCG $(11+13)$

5) GCTGAGATGACTCATCCTCCCAGGCACAGCAAGAAGAAACAGGCAGCATGCCCATTG CTTGTTTATTCCTTGTTTTAATTTTCATTATTACGTTTCTCAGGAAATGCACGCCTT AGCTAACAGTTACTGGCAAAAAAATAAATAAATAAATAAGGTGTTTGAGTTCTTGCC AACATCTGGGGAAGCTGAATGAGATGCCTAGAGGAGGGTTGGCCCATCCTTTGCTTA AAGTTTTCTTTCCTTTTTTTCTTTTTTGTTTTTTTTCTGTCCTTTGGCCCATGAAGA GCATCATGCTTTTGAAGGTGGACGTGGAAAACACTAATTGCACTCTGTAAAGAATTC TTTGCCCTTTGCTGATTGATTTAGGAAACGGTTTTAACTG (9+15)

6) ACTACGGAGGGGCTAGAGCAACAAGT $(35+29)$

7) ACTACGGAGGGGCTAGAGCAACAAGT $(35+29)$

8) ACTACGGAGGGGCTAGAGCAACAAGT $(35+29)$

9) GCTGAGACGACTCATCCTCCCAGGCACAGCAAGAAGAAACAGGGCAGCAGGCCCATT GCTTGTTTATTCCTTGTTTTAATTTTTATTATTACGTTTCTCAGGAAATGCACGCCT TAGCTAACAGTTACTGGCAAAAAAATAAATAAATAAATAAGGTGTTTGAGTTCTTGC CAACATTTGGGGAAGCTGAATGAGATGCCTAGAGGAGGGTTGGCCCATCCTTTGCTT AAAGTTTTCTTTCCTTTTTTTCTTTTTTGTTTTTTTTCTGTCCTTTGGCCCATGAAG AGCATCATGCTTTTGAAGGTGGACGTGGAAAACACTAATTGCACTCTGTAAAGAATT CTTTGTCCTTTGCTGATTGGTTTTGGAAACGGTCTTAACAGGAGGGGGAGTGAAGAG AAGACTTGCCG $(11+13)$

10) ACTACGGAGGGGCTAGAGCAACAAGT $(35+29)$

11) GCTGAGATGACTCATCCTCCCAGGCACAGCAAGAAGAAACAGGCAGCATGCCCATTG CTTGTTTATTCCTTGTTTTAATTTTCATTATTACGTTTCTCAGGAAATGCACGCCTT AGCTAACAGTTACTGGCAAAAAAATAAATAAATAAATAAGGTGTTTGAGTTCTTGCC AACATTTGGGGAAGCTGAATGAGATGCCTAGAGGAGGGTTGGCCCATCCTTTGCTTA AAGTTTTCTTTCCTTTTTTTCTTTTTTGTTTTTTTTCTGTCCTTTGGCCCATGAAGA GCATCATGCTTTTGAAGGTGGACGTGGAAAACACTAATTGCACTCTGTAAAGAATTC TTTGTCCTTTGCTGATTGGTTTTGGAAACGGTCTTAACAGGAGGGAGAGTGAAGAGA AGACTTGCCG $(11+13)$

12) ACTACGGAGGGGCTAGAGCAACAAGT $(35+29)$

13) ACTACGGAGGGGCTAGAGCAACAAGT $(35+29)$

14) ACTACGGAGGGGCTAGAGCAACAAGT $(35+29)$ 
15) ACTACGGAGGGGCTAGAGCAACAAGT $(35+29)$

16) ACTACGGAGGGGCTAGAGCAACAAGT $(35+29)$

17) CGGCAAGTCTTCTCTTCACTCTCCCTCCTGTTAAGACCGTTTCCAAAACCAATCAGC AAAGGACAAAGAATTCTTTACAGAGTGCAATTAGTGTTTTCCACGTCCACCTTCAAA AGCATGATGCTCTTCATGGGCCAAAGGACAGAAAAAAAACAAAAAAGAAAAAAAGGA AAGAAAACTTTAAGCAAAGGATGGGCCAACCCTCCTCTAGGCATCTCATTCAGCTTC CCCAAATGTTGGCAAGAACTCAAACACCTTATTTATTTATTTATTTTTTTGCCAGTA ACTGTTAGCTAAGGCGTGCATTTCCTGAGAAACGTAATAATGAAAATTAAAACAAGG AATAAACAAGCAATGGGCATGCTGCCTGTCTCTTCTTGCTGTGCCTGGGAGGATGAG TCATCTCAGC $(5+15)$

18) ACTACGGAGGGGCTAGAGCAACAAGT $(35+29)$

19) ACTACGGAGGGGCTAGAGCAACAAGT $(35+29)$

20) ACTACGGAGGGGCTAGAGCAACAAGT $(35+29)$

21) ACTACGGAGGGGCTAGAGCAACAAGT $(35+29)$

22) ACTACGGAGGGGCTAGAGCAACAAGT $(35+29)$

23) GCTGAGATGACTCATCCTCCCAGGTACAGCAAGAAGAAATAGGCAGCATGCCCATTG CTTGTTTATTCCTTGTTTTAATTTTCATTATTACGTTTCTCAGGAAATGCACGCCTT AGCTAACAGTTACTGGCAAAAAAATAAATAAATAAATAAGGTGTTTGAGTTCTTGCC AACATTTGGGGAAGCTGAATGAGATGCCTAGAGGAGGGTTGGCCCATCCTTTGCTTA AAGTTTTCTTTCCTTTTTTTCTTTTTTGTTTTTTTTCTGTCCTTTGGCCCATGAAGA GCATCATGCTTTTGAAGGTGGACGTGGAAAACACTAATTGCACTCTGTAAAGAATTC TTTGTCCTTTGCTGATTGGTTTTGGAAACGGTCTTAACAGGAGGGAGAGTGAAGAGA AGACTTGCCG $(11+13)$

24) CGGCAAGTCTTCTCTTCACTCTCCCTCCTGTTAAGATCGTTTCCAAAACCAATCAGC AAAGGACAAAGAATTCTTTACAGAGTGCAATTAGTGTTTTCCACGTCCACCTTCAAA AGCATGATGCTCTTCATGGGCCAAAGGACAGAAAAAAAACAAAAAAGAAAAAAAGGA AAGAAAACTTTAAGCAAAGGATGGGCCAACCCTCCTCTAGGCATCTCATTCAGCTTC CCCAAATGTTGGCAAGAACTCAAACACCTTATTTATTTATTTATTTTTTTGCCAGTA ACTGTTAGCTAAGGCGTGCATTTCCTGAGAAACGTAATAATGAAAATTAAAACAAGG AATAAACAAGCAATGGGCGTGCTGCCTGTTTCTTCTTGCTGTGCCTGGGAGGATGAG TCATCTCAGC $(11+13)$

25) ACTACGGAGGGGCTAGAGCAACAGGT

26) CGGCAAGTCTTCTCTTCACTCTCCCTCCTGTTAAGACCGTTTCCAAAACCAATCAGC AAAGGACAAAGAATTCTTTACAGAGTGCAATTAGTGTTTTCCACGTCCACCTTCAAA AGCATGATGCTCTTCATGGGCCAAAGGACAGAAAAAAAACAAAAAAGAAAAAAAGGA AAGAAAACTTTAAGCAAAGGATGGGCCAACCCTCCTCTAGGCATCCCATTCAGCTTC CCCAAATGTTGGCAAGAACTCAAACACCTTATTTATTTATTTATTTTTTTGCCAGTA ACTGTTAGCTAAGGCGCGCATTTCCTGAGAAACGTAATAATGAAAATTAAAACAAGG AATAAACAAGCAATGGGCATGCTGCCTGTTTCTTCTTGCTGTGCCCGGGAGGATGAG TCATCTCAGC $(5+15)$

27) ACTACGGAGGGGCTAGAGCAACAAGT (35+29)

28) GCTGAGATGACTCATCCTCCCAGGCACAGCAAGAAGAAACAGGCAGCATGCCCATTG CTTGTTTATTCCTTGTTTTAATTTTCATTATTACGTTTCTCAGGAAATGCACGCCTT AGCTAACAGTTACTGGCAAAAAAATAAATAAATAAATAAGGTGTTTGAGTTCTTGCC AACATTTGGGGAAGCTGAATGAGATGCCTAGAGGAGGGTTGGCCCATCCTTTGCTTA AAGTTTTCTTTCCTTTTTTTCTTTTTTGTTTTTTTTCTGTCCTTTAGCCCATGAAGA 
GCATCATGCTTTTGAAGGTGGACGTGGAAAACACTAATTGCACTCTGTAAAGAATTC TTTGTCCTTTGCTGATTGGTTTTGGAAACGGTCTTAACAGGAGGGAGAGTGAAGAGA AGACTTGCCG $(11+13)$

29) GCTGAGATGACTCATCCTCCCAGGCACAGCAAGAAGAAACAGGCAGCATGCCCATTG CTTGTTTATTCCTTGTTTTAATTTTCATTATTACGTTTCTCAGGAAATGCACGCCTT AGCTAACAGTTACTGGCAAAAAATAAATAAATAAATAAGGTGTTTGAGTTCTTGCCA ACATTTGGGGAAGCTGAATGAGATGCCTAGAGGAGGGTTGGCCCATCCTTTGCTTAA AGTTTTCTTTCCTTTTTTCCTTTTTGTTTTTTTTCTGTCCTTTGGCCCATGAAGAGC ATCATGCTTTTGAAGGTGGACGTGGAAAACACTAATTGCACTCTGTAAAGAATTCTT TGTCCTTTGCTGATTGGTTTTGGAAACGGTCTTAACAGGAGGGAGAGTGAAGAGAAG ACTTGCCG $(11+13)$

30) ACTACGGAGGGGCTAGAGCAACAAGT $(35+29)$

31) ACTACGGAGGGGCTAGAGCAACAAGT (35+29)

32) GCTGAGATGACTCATCCTCCCAGGCACAGCAAGAAGAAACAGGCAGCATGCCCATTG CTTGTTTATTCCTTGTTTTAATTTTCATTATTACGTTTCTCAGGAAATGCACGCCTT AGCTAACAGTTACTGGCAAAAAAATAAATAAATAAATAAGGTGTTTGAGTTCTTGCC AACATTTGGGGAAGCTGAATGAGATGCCTAGAGGAGGGTTGGCCCATCCTTTGCTTA AAGTTTTCTTTCCTTTTTTTCTTTTTTGTTTTTTTTCTGTCCTTTAGCCCATGAAGA GCATCATGCTTTTGAAGGTGGACGTGGAAAACACTAATTGCACTCTGTAAAGAATTC TTTGTCCTTTGCTGATTGGTTTTGGAAACGGTCTTAACAGGAGGGAGAGTGAAGAGA AGACTTGCCG $(11+13)$

33) TGTGGTTTTGTTGTTGGGGGGGGGTG

34) ACTACGGAGGGGCTAGAGCAACAAGT (35+29)

35) ACTACGGAGGGGCTAGAGCAACAAGT $(35+29)$

36) ACTACGGAGGGGCTAGAGCAACAAGT $(35+29)$

37) ACTACGGAGGGGCTAGAGCAACAAGT $(35+29)$

38) ACTACGGAGGGGCTAGAGCAACAAGT $(35+29)$

39) ACTACGGAGGGGCTAGAGCAACAAGT $(35+29)$

40) ACTACGGAGGGGCTAGAGCAACAAGT (35+29)

41) TGGGGGGTGTTGTTGTTGTGGTTGTT

42) ACTACGGAGGGGCTAGAGCAACAAGT $(35+29)$

43) ACTACGGAGGGGCTAGAGCAACAAGT (35+29)

44) CGGCAAGTCTTCTCTTCACTCTCCCTCCTGTTAAGACCGTTTCCAAAACCAATCAGC AAAGGACAAAGAATTCTTTACAGAGTGCAATTAGTGTTTTCCACGTCCACCTTCAAA AGCATGATGCTCTTCATGGGCTAAAGGACAGAAAAAAAACAAAAAAGAAAAAAAGGA AAGAAAACTTTAAGCAAAGGATGGGCCAACCCTCCTCTAGGCATCTCATACAGCTTC CCCAAATGTTGGCAAGAACTCAAACACCTTATTTATTTATTTATTTTTTTGCCAGTA ACTGTTAGCTAAGGCGTGCATTTCCTGAGAAACGTAATAATGAAAATTAAAACAAGG AATAAACAAGCAATGGGCATGCTGCCTGTTTCTTCTTGCTGTGCCTGGGAGGATGAG TCATCTCAGC $(5+15)$

45) ACTACGGAGGGGCTAGAGCAACAAGT $(35+29)$

46) GCTGAGATGACTCATCCTCCCAGGTACAGCAAGAAGAAATAGGCAGCATGCCCATTG CTTGTTTATTCCTTGTTTTAATTTTCATTATTACGTTTCTCAGGAAATGCACGCCTT AGCTAACAGTTACTGGCAAAAAAATAAATAAATAAATAAGGTGTTTGAGTTCTTGCC AACATTTGGGGAAGCTGAATGAGATGCCTAGAGGAGGGTTGGCCCATCCTTTGCTTA AAGTTTTCTTTCCTTTTTTTCTTTTTTGTTTTTTTTCTGTCCTTTGGCCCATGAAGA 
GCATCATGCTTTTGAAGGTGGACGTGGAAAACACTAATTGCACTCTGTAAAGAATTC TTTGTCCTTTGCTGATTGGTTTTGGAAACGGTCTTAACAGGAGGGAGAGTGAAGAGA AGACTTGCCG $(11+13)$

47) CGGCAAGTCTTCTCTTCACTCTCCCTCCTGTTAAGACCGTTTCCAAAACCAATCAGC AAAGGACAAAGAATTCTTTACAGAGTGCAATTAGTGTTTTCCACGTCCACCTTCAAA AGCATGATGCTCTTCATGGGCTAAAGGACAGAAAAAAACAAAAAAGAAAAAAAGGAA AGAAAACTTTAAGCAAAGGATGGGCCAACCCTCCTCTAGGCATCTCATTCAGCTTCC CCAAATGTTGGCAAGAACTCAAACACCTTATTTATTTATCTATTTTTTTGCCAGTAA CTGTTAGCTAAGGCGTGCATTTCCTGAGAAACGTAATAATGAAAATTATTACAAGGA ATAAACAAGCAATGGGCATGCTGCCTGTTTCTTCTTGCTGTGCCTGGGAGGATGAGT CATCTCAGC $(5+15)$

48) TGGTTTTGTGTGGGGGGTTGGGTTTG

49) ACTACGGAGGGGCTAGAGCAACAAGT $(35+29)$

50) ACTACGGAGGGGCTAGAGCAACAAGT $(35+29)$

51) ACTACGGAGGGGCTAGAGCAACAAGT $(35+29)$

52) ACTACGGAGGGGCTAGAGCAACAAGT $(35+29)$

53) * GCTGAGATGACTCATCCTCCCAGGCACAGCAAGAAGAAACAGGCAGCATGCCCATT GCTTGTTTATTCCTTGTTTTAATTTTCATTATTACGTTTCTCAGGAAATGCACGCCT TAGCTAACAGTTACTGGCAAAAAAATAAATAAATAAATAAGGTGTTTGAGTTCTTGC CAACATTTGGGGAAGCTGAATGAGATGCCTAGAGGAGGGTTGGCCCATCCTTTGCTT AAAGTTTTCTTTCCTTTTTTTCTTTTTTGTTTTATTTCTGTCCTTTAGCCCATGAAG AGCATCATGCTTTTGAAGGTGGACGTGGAAAACACTAATTGCACTCTGTAAAGAATT CTTTGTCCTTTGCTGATTGGTTTTGGAAACGGTCTTAACAGGAGGGAGAGTGAAGAG AAGACTTGCCG $(11+13)$

54) ACTACGGAGGGGCTAGAGCAACAAGT $(35+29)$

55) ACTACGGAGGGGCTAGAGCAACAAGT $(35+29)$

56) ACTACGGAGGGGCTAGAGCAACAAGT

57) ACTACGGAGGGGCTAGAGCAACAAGT

58) GCTGAGACGACTCATCCTCCCAGGCACAGCAAGAAGAAACAGGGCAGCAGGCCCATT GCTTGTTTATTCCTTGTTTTAATTTTTATTATTACGTTTCTCAGGAAATGCACGCCT TAGCTAACAGTTACTGGCAAAAAAATAAATAAATAAATAAGGTGTTTGAGTTCTTGC CAACATTTGGGGAAGCTGAATGAGATGCCTAGAGGAGGGTTGGCCCATCCTTTGCTT AAAGTTTTCTTTCCTTTTTTTCTTTTTTGTTTTTTTTCTGTCCTTTGGCCCATGAAG AGCATCATGCTTTTGAAGGTGGACGTGGAAAACACTAATTGCACTCTGTAAAGAATT CTTTGTCCTTTGCTGATTGGTTTTGGAAACGGTCTTAACAGGAGGGGGAGTGAAGAG AAGACTTGCCG 


\section{PC3, GT60mer}

\section{Round 1}

1) GGGTTGTTTGgTGTGTTTTTATTGGg

2) GTGGTTGGGGGGGGTGGGGTTTGTTG

3) TTGTgGGTTTGGGTTGGGGGTTTTGG

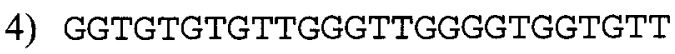

5) TTTGGGGGGGTTGGGGTTTTTTGGGG

6) GGGGTGTTTGGGGTTTGTTGGTGTGG

7) GTTTTTTTTTGGgGtGgTGGGGGGGG

8) GTGTTTTTTGGGGGTGGTGGGGGGGG

9) GTTGtGGGTGTGGTGTTtGTtTGGGT

10) GGGTTGTTGGTTTGTGTTGGGGGTGG

11) *GGGGTGTTTGGGGCTCGTTGGTGTGG

12) GTTTTTGGTTTGGTGGGGTGTGTTGT

\section{Round 2}

1) TGGTGGTGtGTGtGTGTTGGGGGGTG

2) GGGTTTTGTGTGGGGGGGGGGGGTGG

3) GGGTGGGTTTGGGTGTTTGTGTGGGG

4) GGGTtGTGTtgGgGtgGtgtgGgGTT

5) GGGTTGGTTGGTGTGGGAGGTTGTGG

6) TGGTTTTGTGGTTGGTGGTGTGTTGG

7) GTGTGGTTGGGGGGGTGGGTGTGTGT

8) GgGtTTtgGgGGTGGGGTGGTGTTGG

9) TGGTTGGGGGTGGTGGGTGTGGGTTG

10) GGGTGGTGTTTTGGTTGGTGGGTTGT

11) TGGTTTTGGTGTGGTTTGTGGTGTTG

12) TGGGTTTGTGTGGGGGTTGTGTGTTG

13) GGTTGGGGTGGTTGTTGGTTTTGGGT

14) GTTGGTGTTTGGGGGTTTGGGGTGTG

\section{Round 3}

1) GGTGTGTGTGGGGTGGTGTTGGGTGT

2) CAAAAACAGCTTACTTCAGGGACATTTTGGAGCACTGAAATGAACTTATTTGAAAAT TGCAAGAACCACATGAAAATACGGTGACATTTAATGATAAAGTTTATGGATTCGAAG CAAAAACGTCACCTTTAGAAAAGCGAAATCAGAAGCCGCTCACCAGTGGTGTTTAGA GGGGGTTATCATTTGAATCCTACAGAAGAATCATTAAGGTTCTGGGAGGAACATCTG CGGATGTGAGGAAAAAGTCATCGTTATTTTGAAATATTTGATATAAAAAGTTCAATT AGATTCAAAATCCATTTGCATTATCACAAAAAATTCTAACATTATATTTGTCAACGA AAGAAAAAGACGGTTGTTAGATTAGAAGAATGAGTTCAATTCAGAGCAACTGGATTA TTCGAATTCTGGTTTATGGAGAGAAAGGAGGTTTTACCAGGCTGAAACGCAGCCACC ATACTCTCCAGCCATTTGTTCTCAGCTCTTGCTAGAGTTTCTTATCCTCAATCTGGG AAGATTAACCCAGAAATTACCCCAGGCCATTTCACGTAT $(1+7)$ 
3) GCTGAGATGACTCATCCTCCCAGGCACAGCAAGAAGAAACAGGCAGCATGCCCATTG CTTGTTTATTCCTTGTTTTAATTTTCATTATTACGTTTCTCAGGAAATGCACGCCTT AGCTAACAGTTACTGGCAAAAAAATAAATAAATAAATAAGGTGTTTGAGTTCTTGCC AACATTTGGGGAAGCTGAATGAGATGCCTAGAGGAGGGTTGGCCCATCCTTTGCTTA AAGTTTTCTTTCCTTTTTTTCTTTTTTGTTTTTTTTCTGTCCTTTAGCCCATGAAGA GCATCATGCTTTTGAAGGTGGACGTGGAAAACACTAATTGCACTCTGTAAAGAATTC TTTGTCCTTTGCTGATTGGTTTTGGAAACGGTCTTAACAGGAGGGAGAGTGAAGAGA AGACTTGCCG $(1+28)$

4) GGGGTTGGTGTGGTGGTTTAgTGTTG

5) GGTTGGGGGGGGGTTGTTGTGGGGGG

6) GGTGGGTGTTGTTGTTTGTGGTGTTG *

\section{Round 4}

1) GGGGTGTGGGGTTTGGGGGGGTGTTT

2) GTtgGgGgGgGtTTGtgGgGTtGTTT (2)

3) CTAAAACAGCTTACTTCAGGGACATTTTGGGCACTGAAATGAACTTATTTGAAAATT GCAAGAACCACATGAAAATACCGTGACATTTAATGATAAAGTTTATGGATTCCAACC AGAAACATCCCCTTTTCAAAACCGAAATCAGAAGCCGCTCACCACTGGTGTTTAGAG GGGGTTATCATTTGAATCCTACACAACAATCATTATCGTTCTGGGAGGAACATCTGC GGATGTGGGGAAGAAGTCATCGTTATTTTGAAATATTTGATATAAAAAGTTCAATTA GATTCAAAATCCAGTTGCATTATCACAAAAAATTCTAACATTAAATTTGTCCCCGAA ACAAAAAGACGGTTGCTAGATTATAAGAATGAGTTCAATTCACAGCAACTGGATTAT TCGAATTCTGGTTTATGGAGAGAAAGGAGGTTTTACCATTCTGAAACACACCCACCA TACTCTCCTGCCAGTTGTTCTCATCTCTTGCGAGAGTTTCTTATCCTCAATCTGTGA AGATTAACCCTCAAATTACCCCCCTGCCATTTCACGTAT* $(5+3)$

4) GGTGTTTTTGGGGTTGTGGGGTTGGG*

5) TGgGtGTGGGTGTTGTTTGTtGTGTG (2)

6) GgTtggtTTGTTTGTgGgGgGgTTtg

7) GTTGGGGGGGGTTTGTGGGGTTGTTT (2)

8) CAAAAACAGCTTACTTCAGGGACATTTTGGAGCACTGAAATGAACTTATTTGAAAAT TGCAAGAACCACATGAAAATACGGTGACATTTAATGATAAAGTTTATGGATTCGAAG CAAAAACGTCACCTTTAGAAAAGCGAAATCAGAAGCCGCTCACCAGTGGTGTTTAGA GGGGGTTATCATTTGAATCCTACAGAAGAATCATTAAGGTTCTGGGAGGAACATCTG CGGATGTGAGGAAGAAGTCATCGTTATTTTGAAATATTTGATATAAAAAGTTCAATT AGATTCAAAATCCATTTGCATTATCACAAAAAATTCTAACATTATATTTGTCAACGA AAGAAAGAGACGGTTGTTAGATTAGAAGAATGAGTTCAATTCAGAGCAACTGGATTA TTCGAATTCTGGTTTATGGAGAGAAAGGAGGTTTTACCAGGCTGAAACGCAGCCACC ATACTCTCCAGCCATTTGTTCTCAGCTCTTGCTAGAGTTTCTTATCCTCAATCTGGG AAGATTAACCCAGAAATTACCCCAGGCCATTTCACGTAT (5+3)

9) CAAAAACAGCTTACTTCAGGGACATTTTGGAGCACTGAAATGAACTTATTTGAAAAT TGCAAGAACCACATGAAAATACGGTGACATTTAATGATAAAGTTTATGGATTCGAAG CAAAAACGTCACCTTTAGAAAAGCGAAATCAGAAGCCGCTCACCAGTGGTGTTTAGA GGGGGTTATCATTTGAATCCTACAGAAGAATCATTAAGGTTCTGGGAGGAACATCTG CGGATGTGAGGAAGAAGTCATCGTTATTTTGAAATATTTGATATAAAAAGTTCAATT AGATTCAAAATCCATTTGCATTATCACAAAAAATTCTAACATTATATTTGTCAACGA 
AAGAAAGAGACGGTTGTTAGATTAGAAGAATGAGTTCAATTCAGAGCAACTGGATTA TTCGAATTCTGGTTTATGGAGAGAAAGGAGGTTTTACCAGGCTGAAACGCAGCCACC ATACTCTCCAGCCATTTGTTCTCAGCTCTTGCTAGAGTTTCTTATCCTCAATCTGGG AAGATTAACCCAGAAATTACCCCAGGCCATTTCACGTAT (5+3)

10) TGGGTGTGTTTGTGGTTTGGGTGGGG

11) CAAAAACAGCTTACTTCAGGGACATTTTGGAGCACTGAAATGAACTTATTTGAAAAT TGCAAGAACCACATGAAAATACGGTGACATTTAATGATAAAGTTTATGGATTCGAAG CAAAAACGTCACCCTTAGAAAAGCGAAATCAGAAGCCGCTCACCAGTGGTGTTTAGA GGGGGTTATCATTTGAATCCTACAGAAGAATCATTAAGGTTCTGGGAGGAACATCTG CGGATGTGAGGAAAAAGTCATCGTTATTTTGAAATATTTGATATAAAAAGTTCAATT AGATTCAAAATCCATTTGCATTATCACAAGAAATTCTAACATTATATTTGTCAACGA AAGAAAAAGACGGTTGTTAGATTAGAAGAATGAGTTCAATTCAGAGCAACTGGATTA TTCGAATTCTGGTTTATGGAGAGAAAGGAGGTTTTACCAGGCTGAAACGCAGCCACC ATACTCTCCAGCCATTTGTTCTCAGCTCTTGCTAGAGTTTCTTATCCTCAATCTGGG AAGATTAACCCAGAAATTACCCCAGGCCATTTCACGTAT (5+3)

12) GAAAAACAGCTTACTTCGGGGACATTTTGGAGCACTGAAATGAACTTATTTGAAAAT TGCAAGAACCACATGAAAATACGGTGACATTTAATGATAAAGTTTATGGATTCGAAG CAAAAACGTCACCCTTAGAAAAGCGAAATCGAAGCCGCTCACCAGTGGTGTTTAGAG GGGGTTATCATTTGAATCCTACAGAAGAATCATTAAGGTTCTGGGAGGAACATCTGC GGATGTGAGGAAAAAGTCATCGTTATTTTGAAATATTTGATATAAAAAGTTCAATTA GATTCAAAATCCATTTGCATTATCACAAGAAATTCTAACATTATATTTGTCAACGAA AGAAAAAGACGGTTGTTAGATTAGAAGAATGAGTTCAATTCAGAGCAACTGGATTAT TCGAATTCTGGTTTATGGAGAGAAAGGAGGTTTTACCAGGCTGAGACGCAGCCACCA TACTCTCCAGCCATTTGTGCTCAGCTCTTGCTAGAGTTTCTTATCCTCAATCTGGGA TGATTATCCCACATATTACCCCACGCCATGTCAAGTAT* $(5+3)$

13) TTGTTGTTGTGTGTGGTTTGGGGTGT

14) GCTGAGATGACTCATCCTCCCAGGCACAGCAAGAAGAAACAGGCAGCATGCCCATTG CTTGTTTATTCCTTGTTTTAATTTTCATTATTACGTTTCTCAGGAAATGCACGCCTT AGCTAACAGTTACTGGCAAAAAAATAAATAAATAAATAAGGTGTTTGAGTTCTTGCC AACATTTGGGGAAGCTGAATGAGATGCCTAGAGGAGGGTTGGCCCATCCTTTGCTTA AAGTTTTCTTTCCTTTTTTTCTTTTTTGTTTTTTTTCTGTACTTTACCCATGAAGAG CATCATGCTTTTGAAGGTGGACGTGGAAAACACTAAATGCCTTTGTAAAGAATTTTT GTCCTTTGCTGATTGGTTTTTGGAAACGGTCCTTAACAC $(2+26)$

15) GTGTTGGTGGGTTTTTTTGGTGTTTT (2)

16) GTGTTGGTGGGTTTTTTTGGTGTTTT (2)

17) TGGGTGTGGGTGTTGTTTGTTGTGTG (2)

18) GCTGAGATGACTCATCCTCCCAGGCACAGCAAGAAGAAACAGGCAGCATGCCCATTG CTTGTTTATTCCTTGTTTTAATTTTCATTATTACGTTTCTCAGGAAATGCACGCCTT AGCTAACAGTTACTGGCAAAAAAATAAATAAATAAATAAGGTGTTTGAGTTCTTGCC AACATTTGGGGAAGCTGAATGAGATGCCTAGAGGAGGGTTGGCCCATCCTTTGCTTA AAGTTTTCTTTCCTTTTTTTCTTTTTTGTTTTTTTTCTGTCCTTTAGCCCATGAAGA GCATCATGCTTTTGAAGGTGGACGTGGAAAACACTAATTGCACTCTGTAAAGAATTC TTTGTCCTTTGCTGATTGGTTTTGGAAACGGTCTTAACAGGAGGGAGAGTGAAGAGA AGACTTGCCG $(2+26)$

19) GGGTGGTGGGGGTGTGTTGGGGGGTG

20) GTTGAGATGAGTGTTTTGGTTGTTTT* 
21) GGTGGGTGGGTGGTTTTTGTTGTTTT

22) GTTGAGATGAGTGTTTTGGTTTTTTT*

23) TTGGGGTTGGGTGTTTTTTTTGGTGG

24) GCTGAGATGACTCATCCTCCCAGGCACAGCAAGAAGAAACAGGCAGCATGCCCATTG CTTGTTTATTCCTTGTTTTAATTTTCATTATTACGTTTCTCAGGAAATGCACGCCTT AGCTAACAGTTACTGGCAAAAAAATAAATAAATAAATAAGGTGTTTGAGTTCTTGCC AACATTTGGGGAAGCTGAATGAGATGCCTAGAGGAGGGTTGGCCCATCCTTTGCTTA AAGTTTTCTTTCCTTTTTTTCTTTTTTGTTTTTTTTCTGTCCTTTAGCCCATGAAGA GCATCATGTTTTTTATTGTGGACGTGGAAAACACTAATTGCACTCTGTAAAGAATTC TTTGTCCTTTGCTGATTGGTTTTGGAAACGGTCTTAACAGGAGGGAGAGTGAAGAGA AGACTTGCCA

25) GTTGGGTGTGTTGTTTTTGTGGGTTT

\section{Round 5}

1) ATACGTGAAATGGCCTGGGGTAATTTCTGGGTTAATCTTCCCAGATTGAGGATAAGA AACTCTAGCAAGAGCTGAGAACAAATGGCTGGAGAGTATGGTGGCTGCGTTTCAGCC TGGTAAAACCTCCTTTCTCTCCATAAACCAGAATTCGAATAATCCAGTTGCTCTGAA TTGAACTCATTCTTCTAATCTAACAACCGTCTTTTTCTTTCGTTGACAAATATAATG TTAGAATTTTTTGTGATAATGCAAATGGATTTTGAATCTAATTGAACTTTTTATATC AAATATTTCAAAATAACGATGACTTTTTCCTCACATCCGCAGATGTTCCTCCCAGAA CCTTAATGATTCTTCTGTAGGATTCAAATGATAACCCCCTCTAAACACCACTGGTGA GCGGCTTCTGATTTCGCTTTTCTAAAGGTGACGTTTTTGCTTCGAATCCATAAACTT TATCATTAAATGTCACCGTATTTTCATGTGGTTCTTGCAATTTTCAAATAAGTTCAT TTCAGTGCTCCAAAATGTCCCTGAAGTAAGCTGTTTTTG (2)

2) CGGCAAGTCTTCTCTTCACTCTCCCTCCTGTTAAGACCGTTTCCAAAACCAATCAGC AAAGGACAAAGAATTCTTTACAGAGTGCAATTACTGTTTTCCACGTCCACCTTCAAA AGCATGATGCTCTTCATGGGCTAAAGGACAGAAAAAAAACAAAAAAGAAAAAAAGGA AAGAAAACTTTAAGCAAAGGATGGGCCAACCCTCCTCTAGGCATCTCATTCAGCTTC CCCAAATGTTGGCAAGAACTCAAACACCTTATTTATTTATTTATTTTTTTGCCAGTA ACTGTTAGCTAAGGCGTGCATTTCCTGAGAAACGTAATAATGAAAATTAAAACAAGG AATAAACAAGCAATGGGCATGCTGCCTGTTTCTTCTTGCTGTGCCTGGGAGGATGAG TCATCTCAGC $(2+19)$

3) CAAAAACAGCTTACTTCAGGGACATTTTGGAGCACTGAAATGAACTTATTTGAAAAT TGCAAGAACCACATGAAAATACGGTGACATTTAATGATAAAGTTTATGGATTCGAAG CAAAAACGTCACCTTTAGAAAAGCGAAATCAGAAGCCGCTCACCAGTGGTGTTTAGA GGGGGTTATCATTTGAATCCTACAGAAGAATCATTAAGGTTCTGGGAGGAACATCTG CGGATGTGAGGAAAAAGTCATCGTTATTTTGAAATATTTGATATAAAAAGTTCAATT AGATTCAAAATCCATTTGCATTATCACAAAAAATTCTAACATTATATTTGTCAACGA AAGAAAAAGACGGTTGTTAGATTAGAAGAATGAGTTCAATTCAGAGCAACTGGATTA TTCGAATTCTGGTTTATGGAGAGAAAGGAGGTTTTACCAGGCTGAAACGCAGCCACC ATACTCTCCAGCCATTTGTTCTCAGCTCTTGCTAGAGTTTCTTATCCTCAATCTGGG AAGATTAACCCAGAAATTACCCCAGGCCATTTCACGTAT $(2+6)$

4) CAAAAACAGCTTACTTCAGGGACATTTTGGAGCACTGAAATGAACTTATTTGAAAAT TGCAAGAACCACATGAAAATACGGTGACATTTAATGATAAAGTTTATGGATTCGAAG CAAAAACGTCACCTTTAGAAAAGCGAAATCAGAAGCCGCTCACCAGTGGTGTTTAGA GGGGGTTATCATTTGAATCCTACAGAAGAATCATTAAGGTTCTGGGAGGAACATCTG 
CGGATGTGAGGAAAAAGTCATCGTTATTTTGAAATATTTGATATAAAAAGTTCAATT AGATTCAAAATCCATTTGCATTATCACAAAAAATTCTAACATTATATTTGTCAACGA AAGAAAAAGACGGTTGTTAGATTAGAAGAATGAGTTCAATTCAGAGCAACTGGATTA TTCGAATTCTGGTTTATGGAGAGAAAGGAGGTTTTACCAGGCTGAAACGCAGCCACC ATACTCTCCAGCCATTTGTTCTCAGCTCTTGCTAGAGTTTCTTATCCTCAATCTGGG AAGATTAACCCAGAAATTACCCCAGGCCATTTCACGTAT $(2+6)$

5) CGGCAAGTCTTCTCATCACTCTCCCTCCTGTTAAAACCGTTTCCAAAACCAATCAGT TAAGGACAAAGAATTCTTTACAAAGTGCAATTAGTGTTTTCCACGTCCACCTTCTTA ATGATGATGCTCTTCATGGGCTAAAGGACACAAAAAAAACAAAAAACAAAAAAAGGA AAGAAAACTTTAATCAAAGGATGGGCCAACCCTCCTCTAGGCATCTCATTCATCTTC CCCAAATGTTGGCAAGAACTCAAACACCTTATTTATTTATTTATTTTTTTGCCACTA ACTGTTAGCTAAGGCATGCATTTCCTGAAAAGCGTAATAATGAAAATTAAAACAAGG AATAAACAAGCAATGGGCATGCTGCCTGTTTCTTCTTGCTGTGCCTGGGAGGATGAC TCATCTCAGC $(2+19)$

6) CAAGAAACGT

7) GGTGtGTtTgGgGtgGtGgGtgTtGT

8) GCTGAGATGACTCATCCTCCCAGGCACAGCACGAAGAACCAGGCCGCAGGCCCATTG CTTGTTTATTCCTCGTTTTAATTTTCATTATTACGTTTCTCCCGAAATGCACGCCTT AACTAACATTTACGGTTCCAGAAATAAATAAATAAATAAGGTGTTTGACTTCTTGCC AACATTTGGGAAACCTGAATGAGATGCCTAGAGGAGGGTTGGCCCATCCTTTGCTTA ATTTTTCCTTCCTTTTTTTCTTTTTTGTTTTTTTTCTGTCCTTTAGCCCAGGAAGAT GATCTTGCTTTTGAAGGTGGACGTGGAAAACACTAATTGCACTCTGTAAAGAATTCT TTGTCCTTTGCTGATTGGTTTTGGAAATCGTCTTATCAGGAGGGACAATGAATATAA GATCTGTCG* $(1+27)$

9) ATCACGGCATCATC

10) ATACGTGAAATGGCCTGGGGTAATTTCTGGGTTAATCTTCCCAGATTGAGGATAAGA AACTCTAGCAAGAGCTGAGAACAAATGGCTGGAGAGTATGGTGGCTGCGTTTCAGCC TGGTAAAACCTCCTTTCTCTCCATAAACCAGAATTCGAATAATCCAGTTGCTCTGAA TTGAACTCATTCTTCTAATCTAACAACCGTCTTTTTCTTTCGTTGACAAATATAATG TTAGAATTTTTTGTGATAATGCAAATGGATTTTGAATCTAATTGAACTTTTTATATC AAATATTTCAAAATAACGATGACTTTTTCCTCACATCCGCACATGTACTTCCCAGAA CCTTAATGATTCTTCTGTAGGATTCAAATGATAACCCCCTCTAAACACCACTGGTGA GCGGCTTCTGATTTCGCTTTTCTAAAGGTGACGTTTTTGCTTCGAATCCATAGACTT TATCATTAGATGTCAGCGTATATTCATGTGATTCTTGCAATTTTCACATAAGTTCAT TTCTCTGCTCCCAAATGTCGCTGCACTAAGCTGTTTTTG* (2)

11) GTTGGGTGTGTTGGGTGTTTGTGTGG

12) TGGTGATGTGTGGTTGGGTGGTGTGG

13) GCTGAGATGACTCATCCTCCCAGGCACAGCAAGAAGAAACAGGCAGCAGGCCCATTG CTTGTTTATTCCTCGTTTTAATTTTCATTATTACGTTTCTCCCGAAATGCACGCCTT AACTAACATTTACGGTTCCAGAAATAAATAAATAAATAAGGTGTTTGACTTCTTGCC AACATTTGGGAAAGCTGAATGAGATGCCTAGAGGAGGGTTGGCCCATCCTTTGCTTA ATTTTTCCTTCCTTTTTTTCTTTTTTGTTTTTTTTCTGTCCTTTAGCCCAGGAAGAT GATCTTGCTTTTGAAGGTGGACGTGGAAAACACTAATTGCACTCTGTAAAGAATTCT TTGTCCTTTGCTGATTGGTTTTGGAAATCGTCTTATCAGGAGGGACAATGAATATAA GATCTGTCG* 


\section{APPENDIX C \\ Microarray Analysis of Changes in Gene Expression in DU145 Cells Treated with AS1411}

\section{(A) GENES WHOSE EXPRESSION DECREASED AFTER 2 HOURS}

\begin{tabular}{ll}
\hline Fold change & Gene Description \\
\hline-12.0 & calponin homology (CH) domain containing 1 \\
-8.9 & acetyl-Coenzyme A carboxylase alpha \\
-6.8 & B-cell CLL/lymphoma 7C \\
-5.1 & chromosome 6 open reading frame 11 \\
-4.7 & protein kinase C and casein kinase substrate in neurons 3 \\
-4.5 & chromosome 14 open reading frame 34 \\
-3.6 & peptidylprolyl isomerase (cyclophilin)-like 2 \\
-2.8 & autoantigen \\
-2.7 & cholinergic receptor, nicotinic, epsilon polypeptide \\
-2.7 & keratin 15 \\
-2.4 & hypothetical protein MGC5178 \\
-2.3 & hypothetical protein 24432 \\
-2.3 & transmembrane 4 superfamily member 7 \\
-2.2 & hypothetical protein FLJ22341 \\
-2.2 & host cell factor C1 regulator 1 (XPO1 dependant) \\
-2.2 & 7-dehydrocholesterol reductase \\
-2.2 & transmembrane 7 superfamily member 2 \\
-2.1 & pleiomorphic adenoma gene-like 1 \\
-2.1 & proline dehydrogenase (oxidase) 1 \\
-2.1 & PISC domain containing hypothetical protein \\
-2.1 & inhibitor of DNA binding 2, dominant negative helix-loop-helix protein \\
-2.1 & jagged 2 \\
-2.1 & hepatitis delta antigen-interacting protein A \\
-2.1 & stearoyl-CoA desaturase (delta-9-desaturase) \\
-2.0 & filamin B, beta (actin binding protein 278) \\
-2.0 & hypothetical protein FLJ21347 \\
\hline
\end{tabular}




\section{(B) GENES WHOSE EXPRESSION INCREASED AFTER 2 HOURS}

\begin{tabular}{|c|c|}
\hline Fold change & Gene Description \\
\hline 17.4 & Homo sapiens clone 24540 mRNA sequence \\
\hline 11.7 & RAB9, member RAS oncogene family, pseudogene 1 \\
\hline 8.3 & nuclear antigen Sp100 \\
\hline 7.0 & EGF-like repeats and discoidin I-like domains 3 \\
\hline 6.1 & KIAA1068 protein \\
\hline 4.9 & $\begin{array}{l}\text { Homo sapiens mRNA; cDNA DKFZp434J193 (from clone DKFZp434J193); partial } \\
\text { cds }\end{array}$ \\
\hline 4.9 & thymus high mobility group box protein TOX \\
\hline 4.0 & HIV-1 inducer of short transcripts binding protein \\
\hline 4.0 & ADP-ribosylation factor interacting protein 1 (arfaptin 1) \\
\hline 3.2 & likely ortholog of mouse and zebrafish forebrain embryonic zinc finger-like \\
\hline 2.9 & I factor (complement) \\
\hline 2.8 & $\begin{array}{l}\text { TAF6-like RNA polymerase II, p300/CBP-associated factor (PCAF)-associated } \\
\text { factor, } 65 \mathrm{kDa}\end{array}$ \\
\hline 2.8 & 21383_at \\
\hline 2.8 & hypothetical protein MGC11266 \\
\hline 2.6 & hypothetical protein FLJ11142 \\
\hline 2.6 & macrophage stimulating, pseudogene 9 \\
\hline 2.6 & hypothetical protein FLJ32389 \\
\hline 2.5 & leukocyte Ig-like receptor 9 \\
\hline 2.5 & 216688_at \\
\hline 2.4 & zinc finger protein, Y-linked \\
\hline 2.3 & hypothetical protein FLJ13646 \\
\hline 2.3 & eukaryotic translation initiation factor $4 \mathrm{E}$ \\
\hline 2.3 & APG12 autophagy 12 -like (S. cerevisiae) \\
\hline 2.3 & zinc finger protein 45 (a Kruppel-associated box (KRAB) domain polypeptide) \\
\hline 2.2 & polymerase delta interacting protein 46 \\
\hline 2.2 & F-box and WD-40 domain protein 1B \\
\hline 2.2 & amiloride binding protein 1 (amine oxidase (copper-containing)) \\
\hline 2.2 & RNA binding motif protein 3 \\
\hline 2.2 & CD34 antigen \\
\hline 2.1 & nescient helix loop helix 2 \\
\hline 2.1 & 211074_at \\
\hline 2.1 & 211506_s_at \\
\hline 2.1 & transient receptor potential cation channel, subfamily A, member 1 \\
\hline 2.1 & protein tyrosine phosphatase type IVA, member 2 \\
\hline 2.1 & hypothetical protein MGC3067 \\
\hline 2.0 & $\begin{array}{l}\text { solute carrier family } 35 \text { (UDP-N-acetylglucosamine (UDP-GlcNAc) transporter), } \\
\text { member A3 }\end{array}$ \\
\hline 2.0 & superoxide dismutase 2 , mitochondrial \\
\hline
\end{tabular}




\section{(C) GENES WHOSE EXPRESSION DECREASED AFTER 18 HOURS}

\begin{tabular}{|c|c|}
\hline Fold change & Gene Description \\
\hline-78.8 & T-box 1 \\
\hline-62.2 & semenogelin II \\
\hline-27.3 & achaete-scute complex-like 2 (Drosophila) \\
\hline-27.1 & hypothetical protein LOC157697 \\
\hline-20.5 & tumor-associated calcium signal transducer 2 \\
\hline-15.5 & $\begin{array}{l}\text { Homo sapiens similar to dJ309K20.1.1 (novel protein similar to dysferlin, isoform } \\
\text { 1) (LOC375095), mRNA }\end{array}$ \\
\hline-15.2 & 208278_s_at \\
\hline-13.5 & 216737_at \\
\hline-12.4 & single-minded homolog 2 (Drosophila) \\
\hline-12.3 & 217451_at \\
\hline-12.0 & EphA5 \\
\hline-11.6 & $\begin{array}{l}\text { Homo sapiens transcribed sequence with weak similarity to protein } \\
\text { ref:NP_060219.1 (H.sapiens) hypothetical protein FLJ20294 [Homo sapiens] }\end{array}$ \\
\hline-11.6 & $21709 \overline{3}_{\text {_at }}$ at \\
\hline-11.1 & superoxide dismutase 2 , mitochondrial \\
\hline-11.0 & insulin-like growth factor 1 (somatomedin C) \\
\hline-10.8 & $\begin{array}{l}\text { Homo sapiens transcribed sequence with moderate similarity to protein } \\
\text { ref:NP_060219.1 (H.sapiens) hypothetical protein FLJ20294 [Homo sapiens] }\end{array}$ \\
\hline-10.1 & Homo sapiens transcribed sequences \\
\hline-9.8 & histamine receptor $\mathrm{H} 3$ \\
\hline-9.5 & alkaline phosphatase, placental-like 2 \\
\hline-9.4 & G protein-coupled receptor 17 \\
\hline-9.4 & cardiac ankyrin repeat kinase \\
\hline-8.6 & dachshund homolog (Drosophila) \\
\hline-8.4 & A kinase (PRKA) anchor protein 5 \\
\hline-8.3 & ankyrin repeat domain 1 (cardiac muscle) \\
\hline-8.1 & estrogen receptor 1 \\
\hline-8.0 & tight junction protein 3 (zona occludens 3 ) \\
\hline-7.6 & transmembrane protease, serine 4 \\
\hline-7.6 & cold autoinflammatory syndrome 1 \\
\hline-7.5 & glutathione S-transferase theta 2 \\
\hline-7.2 & glutamate receptor, ionotropic, $\mathrm{N}$-methyl D-aspartate 1 \\
\hline-7.1 & hypothetical protein FLJ10786 \\
\hline-6.8 & CD1E antigen, e polypeptide \\
\hline-6.6 & zinc finger protein 157 (HZF22) \\
\hline-6.6 & Homo sapiens cDNA: FLJ21911 fis, clone HEP03855 \\
\hline-6.5 & hypothetical protein FLJ22688 \\
\hline-6.5 & $\begin{array}{l}\text { tissue inhibitor of metalloproteinase } 3 \text { (Sorsby fundus dystrophy, } \\
\text { pseudoinflammatory) }\end{array}$ \\
\hline-6.4 & major histocompatibility complex, class II, DO beta \\
\hline-6.4 & gasdermin-like \\
\hline-6.3 & inversin \\
\hline-6.0 & KIAA0685 \\
\hline-5.9 & small muscle protein, $\mathrm{X}$-linked \\
\hline-5.8 & zinc finger protein 254 \\
\hline-5.7 & $\begin{array}{l}\text { cadherin, EGF LAG seven-pass G-type receptor } 1 \text { (flamingo homolog, } \\
\text { Drosophila) }\end{array}$ \\
\hline-5.7 & telomerase reverse transcriptase \\
\hline-5.5 & Nef associated protein 1 \\
\hline-5.4 & glycoprotein lb (platelet), beta polypeptide \\
\hline
\end{tabular}




\begin{tabular}{|c|c|}
\hline$\overline{-5.1}$ & a disintegrin and metalloproteinase domain 28 \\
\hline-4.9 & high density lipoprotein binding protein (vigilin) \\
\hline-4.9 & NADH:ubiquinone oxidoreductase MLRQ subunit homolog \\
\hline-4.8 & 5-hydroxytryptamine (serotonin) receptor $2 \mathrm{C}$ \\
\hline-4.7 & family with sequence similarity 12 , member $B$ (epididymal) \\
\hline-4.6 & $\begin{array}{l}\text { butyrobetaine (gamma), 2-oxoglutarate dioxygenase (gamma-butyrobetaine } \\
\text { hydroxylase) } 1\end{array}$ \\
\hline-4.5 & tripartite motif-containing 3 \\
\hline-4.4 & $\begin{array}{l}\text { sema domain, immunoglobulin domain (lg), short basic domain, secreted, } \\
\text { (semaphorin) 3F }\end{array}$ \\
\hline-4.4 & 211218 at \\
\hline-4.4 & cathepsin S \\
\hline-4.1 & homeo box D3 \\
\hline-4.1 & FK506 binding protein 12-rapamycin associated protein 1 \\
\hline-3.9 & 217311_at \\
\hline-3.8 & $\begin{array}{l}\text { ubiquitin protein ligase E3A (human papilloma virus E6-associated protein, } \\
\text { Angelman syndrome) }\end{array}$ \\
\hline-3.7 & dystrophin (muscular dystrophy, Duchenne and Becker types) \\
\hline-3.7 & $\begin{array}{l}\text { SWI/SNF related, matrix associated, actin dependent regulator of chromatin, } \\
\text { subfamily a, member } 4\end{array}$ \\
\hline-3.7 & $\begin{array}{l}\text { tyrosine kinase with immunoglobulin and epidermal growth factor homology } \\
\text { domains }\end{array}$ \\
\hline-3.7 & aquaporin 4 \\
\hline-3.6 & forkhead box D3 \\
\hline-3.5 & homeo box A6 \\
\hline-3.4 & adipose specific 2 \\
\hline-3.4 & T-cell leukemia, homeobox 2 \\
\hline-3.4 & caspase recruitment domain family, member 10 \\
\hline-3.3 & ribosomal protein $\mathrm{S} 11$ \\
\hline-3.3 & agouti signaling protein, nonagouti homolog (mouse) \\
\hline-3.3 & arginine vasopressin receptor 2 (nephrogenic diabetes insipidus) \\
\hline-3.2 & diacylglycerol kinase, epsilon $64 \mathrm{kDa}$ \\
\hline-3.0 & eukaryotic translation initiation factor 3 , subunit 5 epsilon, $47 \mathrm{kDa}$ \\
\hline-3.0 & Homo sapiens transcribed sequences \\
\hline-2.9 & granzyme A (granzyme 1, cytotoxic T-lymphocyte-associated serine esterase 3) \\
\hline-2.8 & erythrocyte membrane protein band 4.1 (elliptocytosis $1, \mathrm{RH}$-linked) \\
\hline-2.8 & G protein-coupled receptor 8 \\
\hline-2.8 & potassium inwardly-rectifying channel, subfamily J, member 12 \\
\hline-2.8 & histone 1, $\mathrm{H} 4 \mathrm{f}$ \\
\hline-2.8 & $\begin{array}{l}\text { leukocyte immunoglobulin-like receptor, subfamily A (without TM domain), } \\
\text { member } 5\end{array}$ \\
\hline-2.7 & Homo sapiens transcribed sequences \\
\hline-2.7 & chromodomain helicase DNA binding protein 3 \\
\hline-2.7 & solute carrier family 22 (organic anion/cation transporter), member 11 \\
\hline-2.7 & 221018_s_at \\
\hline-2.6 & ATPase, $\mathrm{H}+$ transporting, lysosomal $9 \mathrm{kDa}, \mathrm{V} 0$ subunit $\mathrm{e}$ \\
\hline-2.6 & fibroblast growth factor 18 \\
\hline-2.6 & LOC92346 \\
\hline-2.6 & Homo sapiens transcribed sequences \\
\hline-2.6 & prostaglandin D2 synthase $21 \mathrm{kDa}$ (brain) \\
\hline-2.5 & KIAA1922 protein \\
\hline-2.5 & hypothetical protein LOC339047 \\
\hline-2.5 & IMP (inosine monophosphate) dehydrogenase 2 \\
\hline-2.5 & Homo sapiens mRNA; cDNA DKFZp564P142 (from clone DKFZp564P142) \\
\hline
\end{tabular}




\begin{tabular}{ll}
\hline-2.4 & transient receptor potential cation channel, subfamily C, member 3 \\
-2.4 & zinc finger protein 165 \\
-2.3 & carnitine palmitoyltransferase 1B (muscle) \\
-2.3 & tripartite motif-containing 31 \\
-2.3 & 221720 s_at \\
-2.3 & leukocyte immunoglobulin-like receptor, subfamily B (with TM and ITIM \\
& domains), member 1 \\
-2.2 & mitogen-activated protein kinase 8 interacting protein 3 \\
-2.2 & cholinergic receptor, nicotinic, epsilon polypeptide \\
-2.2 & chorionic somatomammotropin hormone-like 1 \\
-2.2 & UDP glycosyltransferase 2 family, polypeptide B17 \\
-2.2 & viperin \\
-2.2 & hypothetical protein FLJ12443 \\
-2.2 & calponin homology (CH) domain containing 1 \\
-2.2 & growth differentiation factor 11 \\
-2.1 & calcium channel, voltage-dependent, L type, alpha 1B subunit \\
-2.1 & CD84 antigen (leukocyte antigen) \\
-2.1 & Cysteine knot superfamily 1, BMP antagonist 1 \\
-2.1 & NAD synthetase 1 \\
-2.1 & growth arrest and DNA-damage-inducible, beta \\
-2.1 & ribosomal protein L17 \\
-2.1 & hypothetical protein HSPC109 \\
-2.0 & chromosome 12 open reading frame 6 \\
-2.0 & CDC28 protein kinase regulatory subunit 1B \\
-2.0 & interleukin 24 \\
-2.0 & DEAD/H (Asp-Glu-Ala-Asp/His) box polypeptide 11 (CHL1-like helicase homolog, \\
-2.0 & S. cerevisiae) \\
-2.0 & E4F transcription factor 1 \\
\hline
\end{tabular}




\section{(D) GENES WHOSE EXPRESSION INCREASED AFTER 18 HOURS}

\begin{tabular}{|c|c|}
\hline Fold change & Gene Description \\
\hline 15.6 & HUS1 checkpoint homolog (S. pombe) \\
\hline 14.5 & hypothetical protein FLJ10849 \\
\hline 13.5 & hypothetical protein FLJ10970 \\
\hline 13.2 & DEAD (Asp-Glu-Ala-Asp) box polypeptide 3, X-linked \\
\hline 10.9 & EGF-like repeats and discoidin I-like domains 3 \\
\hline 10.1 & $\begin{array}{l}\text { SWI/SNF related, matrix associated, actin dependent regulator of chromatin, } \\
\text { subfamily a, member } 2\end{array}$ \\
\hline 8.0 & hypothetical protein PRO1853 \\
\hline 6.5 & PTB domain adaptor protein CED-6 \\
\hline 6.2 & SEC10-like 1 (S. cerevisiae) \\
\hline 5.9 & v-rel reticuloendotheliosis viral oncogene homolog (avian) \\
\hline 5.5 & glucosamine ( $\mathrm{N}$-acetyl)-6-sulfatase (Sanfilippo disease IIID) \\
\hline 5.5 & RAB3B, member RAS oncogene family \\
\hline 5.4 & golgi SNAP receptor complex member 2 \\
\hline 5.2 & zinc finger protein $37 a(K O X 21)$ \\
\hline 5.2 & hypothetical protein FLJ12994 \\
\hline 5.1 & prenylcysteine oxidase 1 \\
\hline 5.0 & ATPase, Ca++ transporting, cardiac muscle, slow twitch 2 \\
\hline 4.9 & actin filament associated protein \\
\hline 4.9 & wingless-type MMTV integration site family, member 7B \\
\hline 4.4 & DEAD (Asp-Glu-Ala-Asp) box polypeptide 17 \\
\hline 4.3 & zinc finger RNA binding protein \\
\hline 4.1 & paraneoplastic antigen \\
\hline 4.0 & PTK9 protein tyrosine kinase 9 \\
\hline 3.8 & 211506_s_at \\
\hline 3.7 & 216383_at \\
\hline 3.6 & similar to Caenorhabditis elegans protein $\mathrm{C} 42 \mathrm{C} 1.9$ \\
\hline 3.5 & $\begin{array}{l}\text { guanine nucleotide binding protein ( } G \text { protein), alpha activating activity } \\
\text { polypeptide, olfactory type }\end{array}$ \\
\hline 3.4 & suppression of tumorigenicity \\
\hline 3.3 & Homo sapiens cDNA FLJ31439 fis, clone NT2NE2000707. \\
\hline 3.3 & $\begin{array}{l}\text { tumor necrosis factor receptor superfamily, member } 10 \mathrm{~d} \text {, decoy with truncatec } \\
\text { death domain }\end{array}$ \\
\hline 3.2 & ring finger protein 125 \\
\hline 3.1 & fumarate hydratase \\
\hline 3.1 & stress-induced-phosphoprotein 1 (Hsp70/Hsp90-organizing protein) \\
\hline 3.1 & zinc finger RNA binding protein \\
\hline 3.1 & NGFI-A binding protein 1 (EGR1 binding protein 1) \\
\hline 3.0 & paternally expressed 10 \\
\hline 3.0 & poly (A) polymerase alpha \\
\hline 3.0 & steroid sulfatase (microsomal), arylsulfatase $C$, isozyme $S$ \\
\hline 3.0 & Homo sapiens, clone IMAGE:5294815, mRNA \\
\hline 2.9 & secretory carrier membrane protein 1 \\
\hline 2.9 & endothelial and smooth muscle cell-derived neuropilin-like protein \\
\hline 2.8 & aryl hydrocarbon receptor nuclear translocator-like 2 \\
\hline 2.8 & 208844_at \\
\hline 2.8 & met proto-oncogene (hepatocyte growth factor receptor) \\
\hline 2.8 & SOCS box-containing WD protein SWiP-1 \\
\hline 2.8 & PCTAIRE protein kinase 2 \\
\hline 2.7 & vesicle-associated membrane protein 3 (cellubrevin) \\
\hline
\end{tabular}




\begin{tabular}{|c|c|}
\hline $\begin{array}{l}2.7 \\
2.7\end{array}$ & $\begin{array}{l}\text { Bcl-2-associated transcription factor } \\
\text { cyclin E2 }\end{array}$ \\
\hline 2.7 & hypothetical protein $\mathrm{H} 41$ \\
\hline 2.6 & cell division cycle 27 \\
\hline 2.6 & solute carrier family 7, (cationic amino acid transporter, $y+$ system) member 11 \\
\hline 2.6 & NDRG family member 3 \\
\hline 2.5 & progesterone receptor membrane component 1 \\
\hline 2.5 & mitogen-activated protein kinase kinase kinase kinase 5 \\
\hline 2.5 & zinc finger protein 426 \\
\hline 2.5 & secretory carrier membrane protein 1 \\
\hline 2.5 & heat shock $70 \mathrm{kDa}$ protein 4 \\
\hline 2.5 & APG12 autophagy 12 -like (S. cerevisiae) \\
\hline 2.5 & CD164 antigen, sialomucin \\
\hline 2.5 & AFFX-r2-Hs18SrRNA-M_x_at \\
\hline 2.4 & REV3-like, catalytic subunit of DNA polymerase zeta (yeast) \\
\hline 2.4 & $\begin{array}{l}\text { SWI/SNF related, matrix associated, actin dependent regulator of chromatin, } \\
\text { subfamily a, member } 2\end{array}$ \\
\hline 2.4 & zinc finger protein 45 (a Kruppel-associated box (KRAB) domain polypeptide) \\
\hline 2.4 & septin 10 \\
\hline 2.4 & Homo sapiens hypothetical LOC133993 (LOC133993), mRNA \\
\hline 2.4 & Sec23 homolog A (S. cerevisiae) \\
\hline 2.4 & polymerase (RNA) III (DNA directed) (32kD) \\
\hline 2.4 & hypothetical protein KIAA1164 \\
\hline 2.3 & histone $1, \mathrm{H} 3 \mathrm{~h}$ \\
\hline 2.3 & Ras-GTPase activating protein $\mathrm{SH} 3$ domain-binding protein 2 \\
\hline 2.3 & RIO kinase 3 (yeast) \\
\hline 2.3 & interleukin 6 signal transducer (gp130, oncostatin M receptor) \\
\hline 2.3 & HIV-1 Rev binding protein \\
\hline 2.3 & hypothetical protein MGC3067 \\
\hline 2.3 & calumenin \\
\hline 2.3 & SEC24 related gene family, member D (S. cerevisiae) \\
\hline 2.3 & core-binding factor, beta subunit \\
\hline 2.3 & insulin-like 5 \\
\hline 2.3 & AFFX-HUMRGE/M10098_5_at \\
\hline 2.2 & erythrocyte membrane protein band 4.1-like 1 \\
\hline 2.2 & calumenin \\
\hline 2.2 & butyrate-induced transcript 1 \\
\hline 2.2 & hypothetical protein MGC11061 \\
\hline 2.2 & lectin, mannose-binding, 1 \\
\hline 2.2 & NCK-associated protein 1 \\
\hline 2.2 & RecQ protein-like (DNA helicase Q1-like) \\
\hline 2.2 & chromosome 20 open reading frame 30 \\
\hline 2.2 & secretory carrier membrane protein 1 \\
\hline 2.2 & chromosome 6 open reading frame 62 \\
\hline 2.2 & AFFX-HUMISGF3A/M97935_MA_at \\
\hline 2.1 & calnexin \\
\hline 2.1 & muscleblind-like (Drosophila) \\
\hline 2.1 & SBBI26 protein \\
\hline 2.1 & sphingosine-1-phosphate phosphatase 1 \\
\hline 2.1 & GM2 ganglioside activator protein \\
\hline 2.1 & oculocerebrorenal syndrome of Lowe \\
\hline 2.1 & catalase \\
\hline 2.1 & nucleolar and spindle associated protein 1 \\
\hline
\end{tabular}




\begin{tabular}{ll}
\hline 2.1 & Homo sapiens CDNA FLJ35853 fis, clone TESTI2007078, highly similar to \\
2.1 & MEMBRANE COMPONENT, CHROMOSOME 17, SURFACE MARKER 2. \\
2.1 & DKFZP586N0721 protein \\
2.1 & cleavage and polyadenylation specific factor 5, 25 kDa \\
2.1 & leukocyte-derived arginine aminopeptidase \\
2.1 & transducin (beta)-like 1X-linked \\
2.1 & hypothetical protein MGC14799 \\
2.1 & ROD1 regulator of differentiation 1 (S. pombe) \\
2.1 & promethin \\
2.1 & phosphoglycerate kinase 1 \\
2.1 & M-phase phosphoprotein, mpp8 \\
2.1 & RIO kinase 3 (yeast) \\
2.1 & thioredoxin domain containing \\
2.1 & UDP-Gal:betaGlcNAc beta 1,3-galactosyltransferase, polypeptide 3 \\
2.1 & tumor rejection antigen (gp96) 1 \\
2.0 & PTD016 protein \\
2.0 & Homo sapiens transcribed sequence with weak similarity to protein \\
2.0 & ref:NP_060312.1 (H.sapiens) hypothetical protein FLJ20489 [Homo sapiens] \\
2.0 & 216899_s_at \\
2.0 & AFFX-HUMRGE/M10098_M_at \\
2.0 & solute carrier family 35 (UDP-N-acetylglucosamine (UDP-GlcNAC) transporter), \\
2.0 & member A3 \\
& lamina-associated polypeptide 1B \\
2.0 & hypothetical protein FLJ12806 \\
2.0 & Homo sapiens transcribed sequence with strong similarity to protein \\
2.0 & ref:NP_055485.1 (H.sapiens) basic leucine-zipper protein BZAP45; KIAA0005 \\
\hline & gene product [Homo sapiens] \\
& adenovirus 5 E1A binding protein \\
solute carrier family 16 (monocarboxylic acid transporters), member 1 \\
serum/glucocorticoid regulated kinase-like
\end{tabular}




\section{APPENDIX D}

\section{Abbreviations and Symbols}

AML

AS1411

CD

$\mathrm{CSC}$

CRPC

DNA

ELISA

EMSA

FACS

GRO

MTT

MRCC

$\mathrm{NCI}$

NED

NMR

PCBP

$\mathrm{PrCa}$

$\operatorname{PrCSC}$
Acute myelocytic leukemia

Anticancer aptamer discovered by P. Bates et. al

Circular dichroism

Cancer stem cells

Castration resistant prostate cander

Deoxyribonucleic acid

Enzyme-linked immunosorbent assay

Electromotility shift assay

Fluorescent activated cell sorting

G-rich oligonucleotide

dye for antiproliferation assay

Metastatic renal cell carcinoma

National Cancer Institute

No evidence of disease

Nuclear magnetic resonance

Poly-cytosine binding protein

Prostate cancer

Prostate cancer stem cells 


$\begin{array}{ll}\text { PCR } & \text { Polymerase chain reaction } \\ \text { RNA } & \text { Ribonucleic acid } \\ \text { SELEX } & \text { Systematic Evolution of Ligands by Exponential Enrichment } \\ \text { SP } & \text { Side population } \\ \text { TIC } & \text { Tumor initiating cell } \\ \text { UV } & \text { Ultraviolet } \\ \text { VSELS } & \text { Very small embryonic-like cells }\end{array}$




\section{CURRICULUM VITAE}

\section{ENID W. CHOI}

731 North Hite Avenue, \#5

(c): $\quad 347.306 .2307$

Louisville, KY 40206

(w): $\quad 502.852 .2355$

<ewchoi00@gmail.com>

(f): $\quad 502.852 .2356$

Education

University of Louisville School of Medicine

Louisville, $\mathrm{KY}$

MD Candidate, expected spring 2012. Honors in introduction to clinical medicine, student clinic (director), surgery and pediatric clerkships. Invitation to Golden Key Honour Society (top 15\% of class).

University of Louisville, Dept. of Biochemistry and Molecular Biology

Louisville, KY

PhD, Molecular Biology, expected Fall 2011. Graduate GPA: 4.0/4.0. Thesis: "Novel Tumor-Targeting Aptamers for the Treatment of Prostate Cancer." Thesis supervisor: Dr. Paula J. Bates.

Massachusetts Institute of Technology

BS in Chemical Engineering, Minor in Biology, June 2000.

Cambridge, MA

Research Experience

University of Louisville, Dept. of Biochemistry, Bates Laboratory $\quad$ Louisville, KY

Researcher, July 2006-present

Formulated hypothesis and conducted independent research in the study of cancer stem cells and aptamers (ongoing, publication expected 2011). Characterized cancer-inhibiting and biophysical properties of fourteen DNA oligomer libraries (published 2010). Used SELEX technology to evolve DNA aptamers to inhibit cancer cells in vitro and in vivo. Conducted animal study to test safety and efficacy of a potential anticancer molecule in vivo. Wrote predoctoral research grant proposal (funded by Department of Defense Prostate Cancer Research Project).

Massachusetts General Hospital, Dept. of Oncology, Tanabe Laboratory

Boston, MA

Laboratory Technician, January 2002-August 2004

Studied ability of genetically engineered strains of HSV-1 to target cancer cells. Assisted in refining a new liver metastasis mouse model. Fulfilled safety requirements for clinical trial of one viral strain, including design of PCR assay. Independently engineered a new strain of HSV-1 for in vivo imaging. Designed and standardized common protocols for all laboratory members.

MIT Center for Cancer Research, Hopkins Laboratory

Cambridge, MA Laboratory Technician, August 2000-January 2002

Oversaw and performed physical screening of randomly mutagenized zebrafish families as part of project to determine the developmental role of genes. Identified phenotypically interesting mutations in embryos. Designed primers and performed PCRs to amplify and sequence disrupted genes.

Massachusetts General Hospital, Cutaneous Biology Research Center

Boston, MA Laboratory Technician, April 1997-August 1998

Performed laboratory work involving purification, manipulation, and analysis of skin cell DNA and RNA. 


\author{
Awards and Posters \\ 2004 ULSOM MD/PhD funded fellowship \\ 2006 Institute Representative, American Physician Scientist Association \\ 2007 Dept. of Defense Prostate Cancer Research Predoctoral Fellowship (grant award of \$100,000) \\ 2007 Arno Spatola Graduate Fellowship, Institute for Molecular Diversity and Drug Design \\ 2009 Poster presentation at AACR Annual Meeting \\ 2010 Second place, student poster competition, Kentucky Chapter of American College of Physicians \\ 2010 Third place, student poster competition, James Graham Brown Cancer Center Retreat \\ 2011 Poster presentation at AACR Cancer Stem Cell special interest meeting \\ 2011 Institute for Molecular Diversity and Drug Design Travel Award to AACR meeting \\ 2011 Poster presentation at Dept. of Defense Innovative Minds in Prostate Cancer Today conference
}

\title{
Publications
}

Choi EW, Nayak LV, Bates PJ. Cancer-selective antiproliferative activity is a general property of some Grich oligodeoxynucleotides. Nucleic Acids Res. 2010 Mar;38(5):1623-35. Epub 2009 Dec 11.

Bates PJ, Choi EW, Nayak LV. G-Rich oligonucleotides for cancer treatment. Methods Mol Biol. 2009;542:379-92.

Kasuya H, Kuruppu DK, Donahue JM, Choi EW, Kawasaki H, Tanabe KK. Mouse models of subcutaneous spleen reservoir for multiple portal venous injections to treat liver malignancies. Cancer Res. 2005 May 1;65(9):3823-7.

Kasuya H, Pawlik TM, Mullen JT, Donahue JM, Nakamura H, Chandrasekhar S, Kawasaki H, Choi E, Tanabe KK. Selectivity of an oncolytic herpes simplex virus for cells expressing the DF3/MUC1 antigen. Cancer Res. 2004 Apr 1;64(7):2561-7.

(Acknowledgement) Golling G, Amsterdam A, Sun Z, Antonelli M, Maldonado E, Chen W, Burgess S, Haldi M, Artzt K, Farrington S, Lin SY, Nissen RM, Hopkins N. Insertional mutagenesis in zebrafish rapidly identifies genes essential for early vertebrate development. Nat Genet. 2002 Jun;31(2):135-40. Epub 2002 May 13.

\section{Extracurricular Activities}

University of Louisville Health Outreach and Pediatric Education Clinic Louisville, KY

Clinic Director, January 2005-June 2006

Headed volunteer clinic staffed by students to serve indigent patients. Liaised with attending doctors and scheduled student volunteers. Instructed rotating students in medical recordkeeping and basic medical procedures including phlebotomy, blood glucose testing, and physical exams.

Latin American Studies Internship Supervisor, January 2005-June 2006

Created internship credit opportunity for UL undergraduate students through Spanish language department. Students worked as medical translators in the clinic. Oversaw students' weekly learning experiences, and submitted written evaluations for each student to the department.

\section{The Center for Women and Families}

Louisville, KY

Medical Advocate, January 2008-Present

Completed 5 week training program to qualify as a medical advocate and counselor for victims of sexual assault and domestic violence. Worked on-call sessions on a biweekly basis, volunteered with children and worked for special events on a monthly basis.

University of Louisville School of Medicine Louisville, KY

Dance instructor, October 2004 June 2005

Organized weekly dance lessons for medical students. Choreographed and taught classes in jazz and hiphop, and recruited ballroom and ballet teachers. Organized a one month swing dance workshop.

Knitting instructor, October 2004 June 2005 
Organized and taught weekly knitting lessons for medical students. Created kits of materials and instructions for students. Collected finished handmade items, including baby blankets and hats for donation to "Knit for a Cause," a local charity.

\section{Activities/Interests}

Member AACR, AMSA, Phi Delta Epsilon, ULSOM Oncology Club (Treasurer), Pediatric Club, Unit Lab Advocate, Exam Challenge Committee. Classes with Louisville Ballet, MIT Dance Troupe (6 years; choreographer, 5 years). Korean Students Association (Secretary, Vice President, President), Kappa Alpha Theta, MIT Interfraternity Council (Treasurer), MIT Cheerleading (founding member), Concert Choir. Hobbies include dancing, cooking, running, and knitting. 